\title{
The role of postsynaptic density (PSD) proteins PSD-95 and PSD-93 for mouse visual cortical plasticity and vision
}

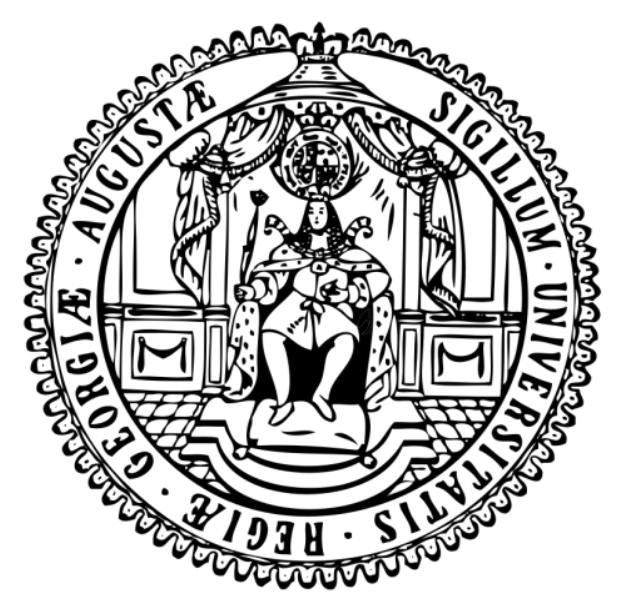

\section{Dissertation}

for the award of the degree

"Doctor rerum naturalium"

of the Georg-August-Universität Göttingen

within the doctoral program Sensory and Motor Neuroscience of

the Göttingen Graduate School for Neurosciences, Biophysics, and Molecular Biosciences (GGNB)

of the Georg-August University School of Science (GAUSS)

submitted by

Sophia Katharina Stodieck

From Münster, Germany

Göttingen 2016 


\section{Examination board}

\section{Thesis Committee Members:}

Prof. Dr. Siegrid Löwel ( $1^{\text {st }}$ Reviewer, advisor)

Systems Neuroscience Group

Johann-Friedrich-Blumenbach-Institute for Zoology und Anthropology

University of Göttingen

Prof. Dr. Tim Gollisch (2 ${ }^{\text {nd }}$ Reviewer)

Sensory Processing in the Retina

Department of Ophthalmology

University Medical Center Göttingen

Prof. Dr. Dr. Oliver Schlüter

Molecular Neurobiology

European Neuroscience Institute Göttingen

\section{Extended Thesis Committee Members:}

Prof. Dr. Ralf Heinrich

Department of Cellular Neurobiology

Schwann-Schleiden Research Center Göttingen

Prof. Dr. Tobias Moser

Institute for Auditory Neuroscience

University Medical Center Göttingen

\section{Dr. Ellen Reisinger}

Department of Otolaryngology

Center for Molecular Physiology of the Brain

University of Göttingen

Date of the oral examination: 21.09.2016 


\section{Table of contents}

\section{Contents}

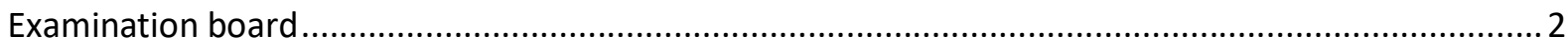

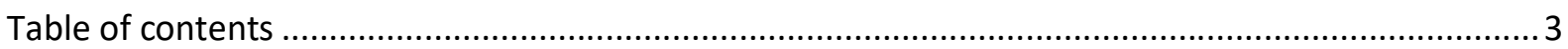

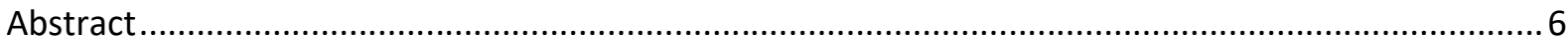

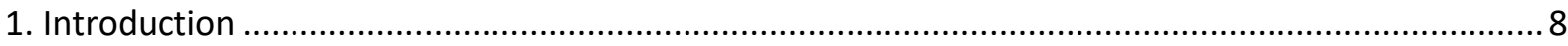

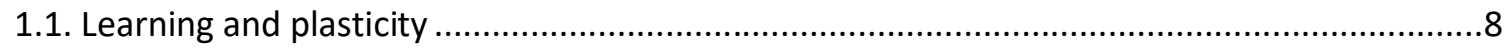

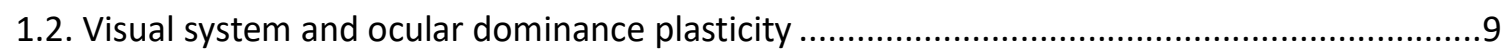

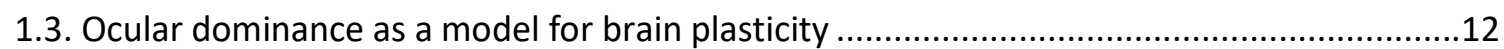

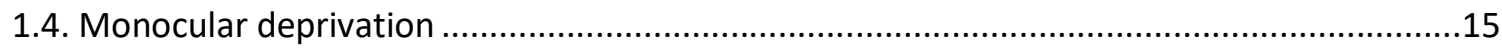

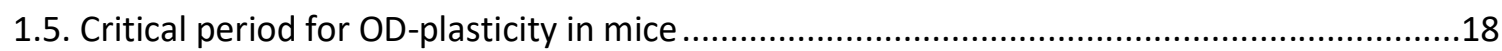

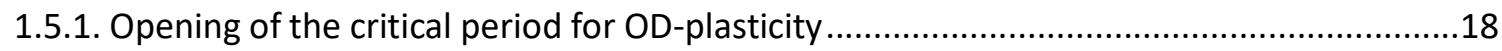

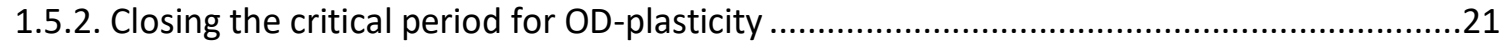

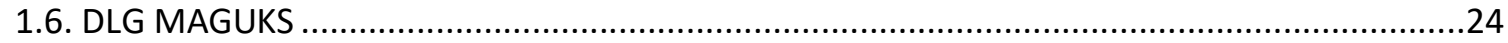

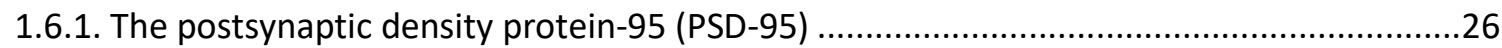

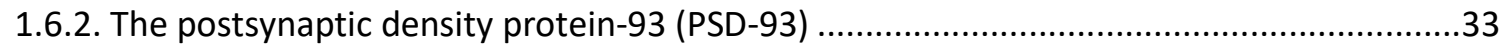

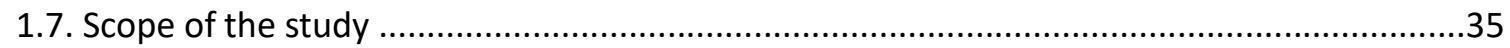

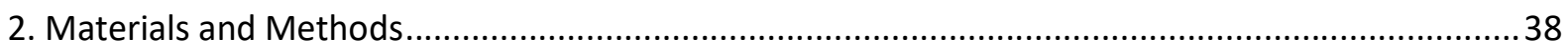

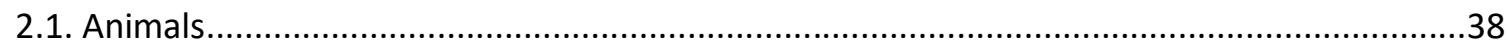

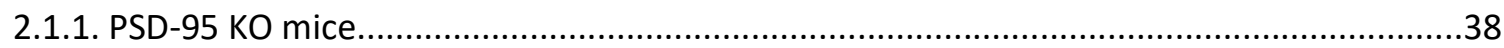

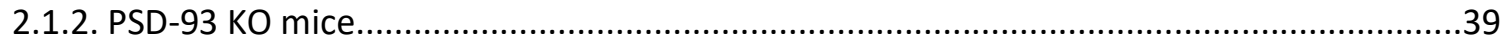

2.2. Visual deprivation - dark rearing of PSD-93 KO and WT mice ............................................39

2.3. Lifelong juvenile-like ocular dominance plasticity in PSD-95 KO mice .................................40

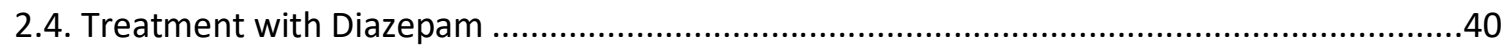

2.5. Neural circuits are less stable in PSD-95 KO mice.............................................................

2.6. V1-specific reduction of PSD-95 expression restores juvenile-like ocular dominance plasticity

2.7. The role of PSD-93 on ocular dominance plasticity and vision in mice ...............................44

2.8. V1-specific reduction of PSD-93 expression in V1 of WT mice from birth ...........................45

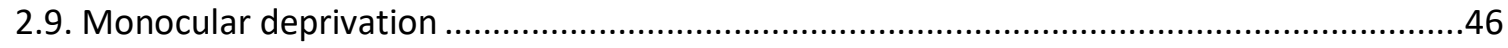

2.10. Quantification of visual capabilities in a behavioral task..................................................47

2.10.1. The virtual-reality optomotor system (Prusky et al., 2004) ..............................................47 


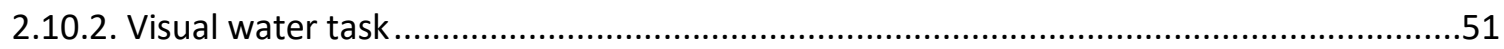

2.10.3. Determination of the maximum spatial frequency threshold........................................52

2.10.4. Determination of the minimum angle difference in the visual water task .......................56

2.11. Surgical preparations for optical imaging of intrinsic signals in vivo ..................................58

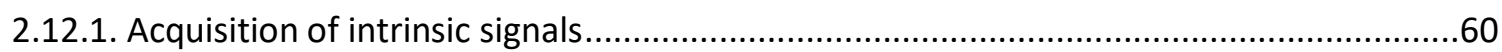

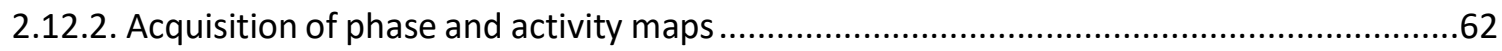

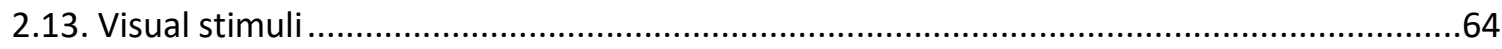

2.13.1. Acquisition of map quality from elevation and azimuth maps.......................................66

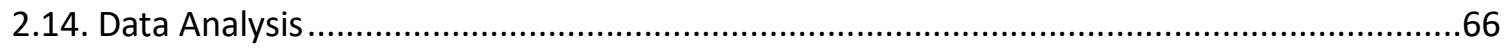

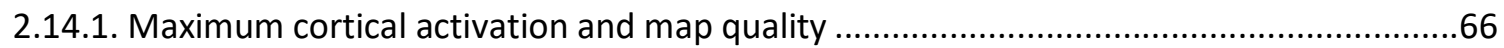

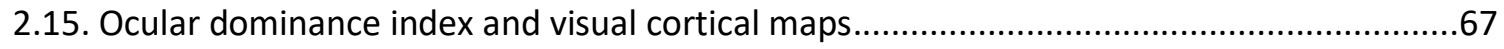

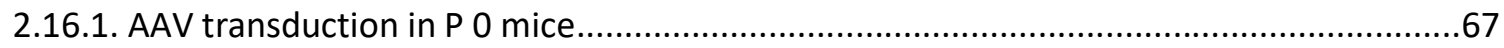

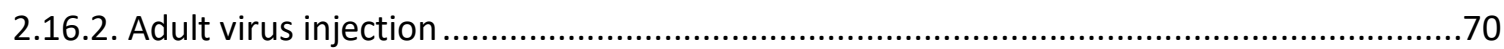

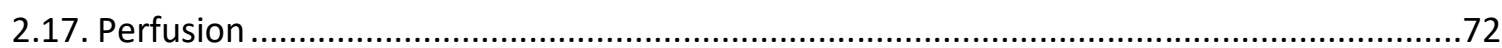

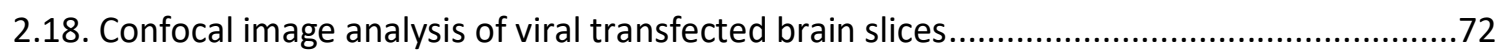

2.19. Analysis of virus transfected brain slices with a fluorescent microscope ...........................73

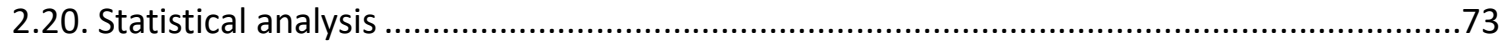

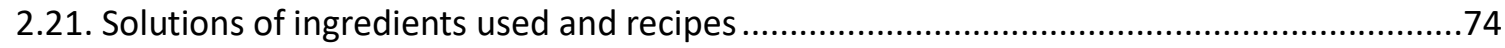

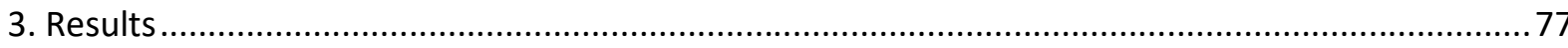

3.1. The juvenile form of OD-plasticity is preserved in PSD-95 KO mice until late adulthood.....77

3.2. Neural circuit changes are less stable in PSD-95 KO mice..... .89

3.3. V1-specific knockdown of PSD-95 expression before maturation (P 0 ) restores juvenile-like

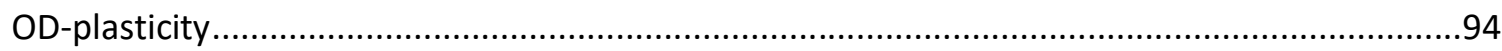

3.4. AAV transduction analysis of V1-specific PSD-95 knockdown and control mice (P 0) ..........99

3.5. V1-specific knockdown of PSD-95 expression after maturation (P 40) restores juvenile-like ODplasticity 100

3.6. AAV transduction analysis of V1-specific PSD-95 knockdown and control mice (P 40) .102

3.7. Normal visual acuity and orientation discrimination before and after a selective knockdown of PSD-95 in the visual cortex of WT mice 103

3.8. V1-specific knockdown of PSD-95 expression after the phase for OD-plasticity (beyond P 110; Löwel \& Lehmann, 2008) restores juvenile-like OD-plasticity..... 107

3.9. AAV transduction analysis of V1-specific PSD-95 knockdown and control mice (> P 110) .....112 3.10. Visual capabilities measured with the virtual-reality optomotor system in WT mice before and after a visual cortex restricted knockdown of PSD-95 117

3.10.1. Maximum spatial frequency threshold (visual acuity) 117 
3.10.2. Visual acuity increase during 7 days of monocular deprivation ...................................117

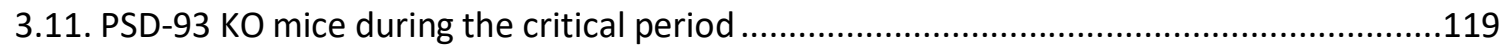

3.11.1. Visual capabilities measured with the virtual-reality optomotor system .......................119

3.11.2. Maximum spatial frequency threshold (visual acuity) .................................................119

3.11.3. Visual acuity increase during four days of monocular deprivation ...............................120

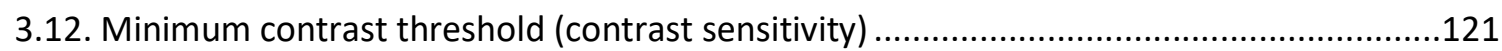

3.12.1. Contrast sensitivity of WT and PSD-93 KO mice during the mid CP ...........................121

3.12.2. Contrast sensitivity of WT and PSD-93 KO mice during the late CP .............................121

3.13. Visual capabilities of PSD-93 KO and WT mice measured in the VWT ...........................123

3.14. OD-plasticity of WT and PSD-93 KO mice during mid critical period................................124

3.15. OD-plasticity of WT and PSD-93 KO mice during late critical period ................................131

3.16. Dark rearing does not prevent the early maturation of PSD-93 KO mice ........................136

3.17. Running does not preserve OD-plasticity in PSD-93 KO mice .143

3.18. Visual capabilities measured with the virtual-reality optomotor system in PSD-93 KO mice after voluntary physical exercise in a running wheel 147

3.18.1. Maximum spatial frequency threshold (visual acuity) .147

3.18.2. Contrast sensitivity of PSD-93 KO mice during the late CP after voluntary physical exercise in a running wheel .148

3.19. Visual cortex restricted knockdown of PSD-93 in WT mice 149

3.20. Visual capabilities measured with the virtual-reality optomotor system in WT mice after a visual cortex restricted knockdown of PSD-93 .154

3.20.1. Maximum spatial frequency threshold (visual acuity) .154

3.20.2. Minimum contrast threshold (contrast sensitivity)..... 155

3.21. Retinotopy and map quality of PSD-93 knockout and WT mice 157

3.22. Retinotopy and map quality of PSD-93 knockdown and control mice .158

3.23. AAV transduction analysis of V1-specific PSD-93 knockdown and control mice (P 0) ......159

4. Discussion

4.1. PSD-95 164

4.2. PSD-93 .173

References 180

Acknowledgment 197 


\section{Abstract}

During neurodevelopment in the brain, several changes including structural, molecular and functional changes take place in order to promote its maturation. These changes occur at multiple levels, with modifications in synaptic transmission, protein expression or the sensitivity to experience-dependent plasticity. In mice, ocular dominance (OD) plasticity is highest during the critical period (CP) between postnatal days (P) 20 - P 35 and decreases afterwards until it is fully absent in mice $>$ P 110 when raised in standard cages.

The predominant view is that excitation and inhibition are the key players in opening and closing the $\mathrm{CP}$. The present $\mathrm{PhD}$ thesis proposes a different mechanism which is based upon the two postsynaptic density proteins (PSD) 93 and 95 of the membrane associated guanylate kinase (MAGUK) protein family. Both scaffold proteins are required for organization of postsynaptic signaling and synaptic transmission in excitatory synapses. PSD-95 was shown to be absolutely required for experience-dependent maturation of AMPA receptor silent synapses in the visual cortex (VC) of mice, whereas PSD-93 might act as an opponent and prevents early synaptic maturation.

To assess the contribution of both proteins in OD-plasticity, PSD-95 and PSD-93 knockout (KO) mice were examined by using monocular deprivation (MD) and optical imaging of intrinsic signals in vivo. In addition, visual abilities of these mice were analyzed with the behavioral tests optomotor system and the visual water task (VWT). PSD-95 KO mice show lifelong juvenile-like OD-plasticity after both 4 and 7 days (d) of MD at least until P 507. Furthermore, pharmacological manipulation of enhancing intracortical inhibition in vivo resulted in juvenilelike OD-plasticity in PSD-95 KO while it was completely prevented in WT mice (<P 110). This suggests that the preserved OD-plasticity in PSD-95 KO mice is caused by a higher fraction of AMPA receptor silent synapses. Under consideration of the generally unstable nature of PSD-95 deficient neurons, a modified OD-plasticity paradigm was designed: After $7 \mathrm{~d}$ of MD, the previously deprived eye was reopened and recovery time of the induced OD- shift was significantly increased (2 d) in PSD-95 KO mice compared to WT. Thus, plastic changes in PSD$95 \mathrm{KO}$ mice are more transient and reverse faster than in WT mice. This indicates that synaptic changes during experience-dependent network refinements cannot be consolidated and functional properties of neurons may not be stabilized without PSD-95.

To narrow down the exact location of OD-plasticity expression, a VC-restricted knockdown 
(KD) of PSD-95 was conducted in P 0, P 40 or > P 140 WT mice. OD-plasticity was present after $4 \mathrm{~d}$ of $\mathrm{MD}$ in all tested KD animals and the location of its expression is confined to the contralateral VC of the deprived eye. Together, these results support a conceptual model of PSD-95-dependent silent synapse maturation which eventually leads to the ending of the CP for OD-plasticity.

Contemporaneously to this project, a KO of PSD-93 was shown to evoke a precocious maturation of silent synapses in the VC. Likewise, an earlier closure of the CP (> P 27) was observed in this work for OD-plasticity in PSD-93 KO and VC-restricted PSD-93 KD mice. Since the sensitive phase for OD-plasticity can be prolonged by rearing animals in the dark (DR) from birth, this effect was studied on PSD-93 KO mice in this project. PSD-93 KO mice completely failed to show OD-plasticity after DR, indicating that the faster maturation of PSD- 93 KO mice is not an experience-dependent process. Recently, it was shown that voluntary physical exercise can promote OD-plasticity in WT mice. The benefit of running was investigated in PSD-93 KO mice during the late CP (> P 28). Surprisingly, even physical exercise failed to induce OD-plasticity, indicating that there is an earlier closure of the CP for OD-plasticity after P 27 in PSD-93 KO mice. Hence, a lack of PSD-93 prevents OD-plasticity which is usually inducible by DR or voluntary physical exercise in WT mice. Moreover, possible effects of PSD-95 or PSD$93 \mathrm{KO}$ on visual perception were tested by subjecting mice to a visual discrimination task (VWT). While visual acuity was normal for both PSD-95 -and PSD-93 KO- mice, orientation discrimination was severely impaired in both genotypes: KO-mice required more than double the orientation contrast for a correct behavioral decision compared to WT mice.

In summary, the presented PhD thesis provides evidence that PSD-95 controls the ending of the CP for juvenile OD-plasticity, whereas PSD-93 exhibits the opposing function. In contrast to the current view that increased intracortical inhibition is the major player in closing the $\mathrm{CP}$, the findings of this PhD study allow the opposing assumption that the closure is mainly based on PSD-95 function and mostly independent of the inhibitory tone. Taken together, these results expand the knowledge about the molecular mechanisms underlying synaptic maturation in VC and enrich the current view concerning the roles of PSD-95 and PSD-93 and its functional interactions regulating OD-plasticity. 


\section{Introduction}

\subsection{Learning and plasticity}

Humans and other animals are able to live and move in their given habitat and to perform complicated tasks such as thinking, decision making and problem solving. Independent of their complexity, these tasks are mainly based on the brain, a very powerful and elaborate neuronal network which consists of billions of neurons (Pelvig et al., 2008). The brain and its ability to learn is very fascinating and a unique phenomenon which led many scientists to investigate how the brain works and what the underlying principles and mechanisms of learning are. One interesting question in neuroscience is how do we actually learn and how does the brain store and recall important information?

Learning requires neuronal plasticity that corresponds to the ability of the brain to reorganize itself, both structurally and functionally. Neuronal plasticity enables the brain to adapt to changes in the environment or cope with pathological conditions like brain injuries. Within the brain, neural cells are connected to each other and 'communicate' via synapses. Synapses allow a neuron to pass either an electrical or chemical signal to another neuron or cell (Foster et al., 1897; Schacter et al., 2011). Early plasticity in young, still developing animals is actually required to shape a functional neural network and is not given but has to be trained. Plasticity is generally highest during early development where ongoing learning experiences are shaping the neuronal circuits. This results in the enhanced adaption to the environment and hence, increases survival.

Sensory processing is a neurological process that allows the brain to organize sensation from the environment and perception of the own body. This sensing and perception makes it possible to process inputs resulting in usable functional outputs (Stein et al., 2009; Stein and Rowland, 2011). From a sensory integrative perspective, learning occurs when an animal receives accurate sensory information, processes it, and uses it to organize its behavior accordingly. Experience-dependent plastic changes occur in many different regions of the brain depending on the sensory input. The nervous system possesses individual sensory systems or organs, which are dedicated specifically to one of the traditionally most recognized senses, namely sight (ophthalmoception), hearing (audioception), taste (gustaoception), smell (olfacoception) or touch (tactioception). 
However, vision is one of the most important senses for many species. It allows sensing the environment to forage or to detect sources of danger like predators, for example.

The main goal of this thesis was to investigate how plastic changes occur in the brain. To analyze this, the visual system of mice was used since it shares many similarities to that of humans and is widely used and well established in Neuroscience.

\subsection{Visual system and ocular dominance plasticity}

In vertebrates, visual information gathered by the eyes has to pass the retino-collicular or the retino-geniculate pathways in the brain until it finally gets processed in the visual cortex. The visual cortex is located in both hemispheres at the back of the brain. In detail, firstly, visual information encoded by photons reaches the retina, a very light-sensitive tissue in the eye and one part of the central nervous system (CNS). The retina absorbs light photons which are converted into electrical signals like action potentials. The multilayered retina is capable of transmitting and processing visual information due to a highly complex cellular network, consisting of special types of photoreceptor cells, so called rods and cones (Figure $1 \mathrm{~A}$ ). The propagation of signals to bipolar and horizontal cells (Figure $1 \mathrm{~A}$ ) is carried out by chemical synapses which release the neurotransmitter glutamate in the dark perpetually. The electrochemical signals in the retina are transferred from photoreceptors via bipolar cells to the retinal ganglion cells. This signal processing is completed by additional horizontal connections, promoting lateral inhibition through horizontal and amacrine cells which are providing 'vertical' links between bipolar and ganglion cells (Figure $1 \mathrm{~A}$ ). The release of neurotransmitter requires a special type of chemical synapses called the ribbon synapses (Figure $1 \mathrm{~B}-\mathrm{E}$ ). The ribbon synapses represent a part of the presynaptic active zone and are characterized by a specific mechanism of vesicle fusion that supports a rapid release of the neurotransmitter glutamate and therefore, signal conveyance. Characteristic features of ribbon synapses are that they are being surrounded by hundreds of synaptic vesicles (RaoMirotznik et al., 1995) and being located in the retina in rods as well as in cones and in bipolar cells (Sjöstrand, 1958; Kidd, 1962; Missotten, 1965; Dowling and Boycott, 1966) (Figure 1 BE). The action potentials are subsequently transmitted along optic nerve fibers from the retina via the thalamus to the visual cortex (VC) of the brain. 


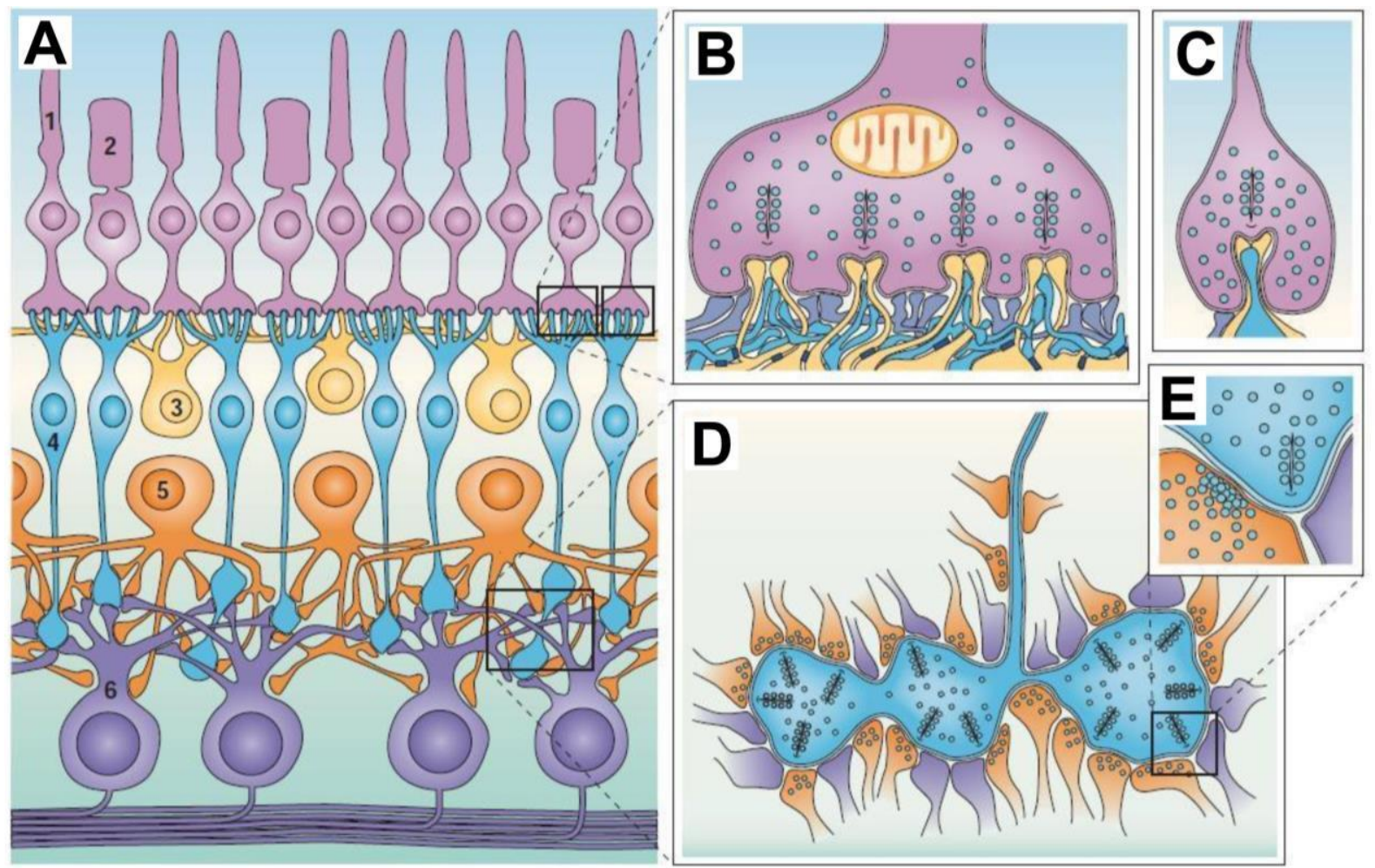

Figure 1: Scheme of the mammalian retina (Figure modified from Wässle, 2004). (A) In the mammalian retina, there are six types of different neurons: rods (1), cones (2), horizontal cells (3), bipolar cells (4), amacrine cells (5) and retinal ganglion cells (6). When light in form of photons reaches a photoreceptor cell, it sends a synaptic response to bipolar cells which in turn processes information to the retinal ganglion cells. The photoreceptors are also connected with horizontal cells and amacrine cells which modify the synaptic signal before it is transferred to the ganglion cells. Rods are mostly active in dim light conditions and intermixed with cone signals that are less sensitive and work best in bright light conditions. (B) A synaptic terminal of a cone. Four presynaptic ribbons are connected to the dendrites of bipolar cells (blue) and horizontal cells (yellow). (C) A synaptic terminal of a rod. Only one presynaptic ribbon is attached to the invaginating axons of bipolar cells (blue) and horizontal cells (yellow). (D) The axon terminal of one bipolar cell (blue) contains up to 50 presynaptic ribbons and connects to postsynaptic amacrine cells (orange) and retinal ganglion cell dendrites (purple). (E) Amplified scheme of a bipolar cell ribbon synapse (blue) with an amacrine cell (orange) and a retinal ganglion cell dendrite (purple). The amacrine cell provides a feedback synapse onto the bipolar cell.

The signal transfer from the retina to the visual cortex is mediated by axons of the retinal ganglion cells that form the optic nerve (nervus opticus). In mice, nasal retinal fibers of the optic nerve (about $80 \%$ of all optic nerve axons) cross to the contralateral hemisphere of the brain in the optic chiasm (chiasma opticum), whereas temporal fibers project to the ipsilateral hemisphere without crossing the optic chiasm (Figure 2) (Dräger and Olsen, 1980). As the majority of nerve axons within optic fibers are projecting to the contralateral hemisphere, the 
visual cortex of mice is dominated by input signals coming from the contralateral eye, which is commonly referred to as 'ocular dominance' (Figure 2). Thus, in mice, only the central $30^{\circ}$ to $40^{\circ}$ of the upper part of each visual hemifield is seen by both eyes (Dräger, 1975; Wagor et al., 1980; Gordon and Stryker, 1996). The lateral geniculate nucleus (LGN) receives information directly from the ascending retinal ganglion cells via the optic tract and neurons of the LGN finally send their axons to the primary visual cortex (V1). In addition, the LGN also obtains feedback connections coming from the primary visual cortex (Cudeiro et al., 2006). Visual stimuli originating from the right visual field activate the left part of the retina, whereas the right part of the retina receives visual information coming from the left visual field. Hence, there is some degree of binocular overlap in the visual field located frontally of the mouse. The spatial arrangement of visual stimuli in the visual field and the resulting stimulation pattern of the retinae are preserved throughout the visual pathway. Consequently, neighboring stimuli in the visual field are also activating adjacent neurons in the $\mathrm{V} 1$. This preservation of the spatial arrangement of visual inputs coming from retina is referred to as retinotopy and a neuronal map of the visual field as a retinotopic map (Wagor et al, 1980; Schuett et al., 2002).

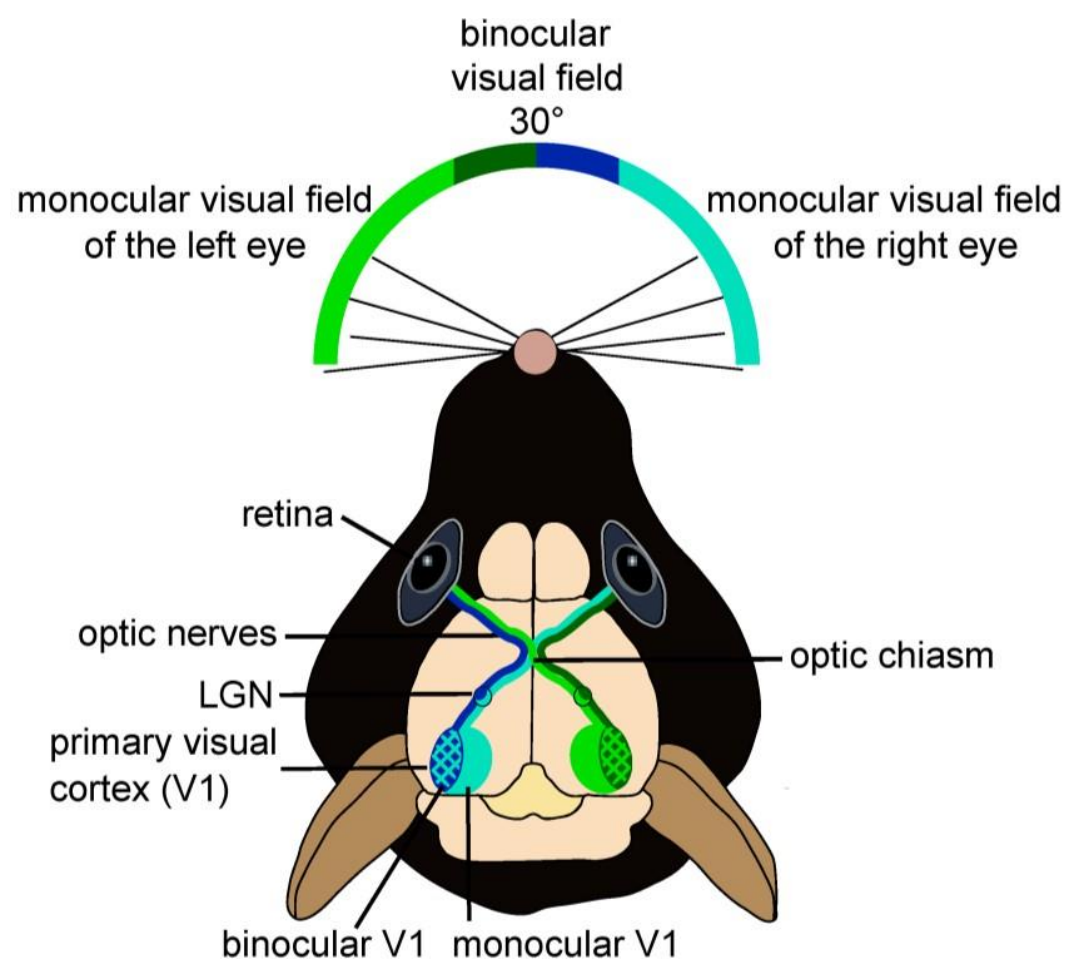

Figure 2: Representation of the mouse visual pathway and its visual field. Left and right visual fields and their respective representations in the visual pathway of the mouse are illustrated with green and blue colors. The visual information originating from the nasal part of the retina crosses to the other hemisphere in the optic chiasm (light blue and light green for right and the left eye, respectively). Visual information from the temporal 
part of the retina propagates within the same hemisphere and does not cross at the optic chiasm (dark blue and dark green for the right and left eye, respectively). Visual information in form of photons is transferred to action potentials in the retina and reaches the lateral geniculate nucleus (LGN) where it is further relayed to the primary visual cortex (V1). While the binocular part of $\mathrm{V} 1$ receives input coming from both eyes, the monocular part of V1 only receives input from the contralateral eye.

The visual cortex is divided into the monocular part which exclusively gets activated by visual stimulation of the contralateral eye only and the binocular part which receives inputs by visual stimulation of both eyes (Dräger, 1975). The monocular region in V1 covers the biggest area and is located at the medial side of the brain. The binocular zone is located at the lateral side of V1 and occupies only about one third of it. The frontal part of the visual field is represented in the retina of both eyes and is therefore located in the binocular zone of V1 (Gordon and Stryker, 1996). Even though the binocular visual cortex of mice receives input signals after visual stimulation of both eyes, it exhibits stronger cortical responses to stimulation of the contralateral eye and weaker responses after ipsilateral eye stimulation (Dräger, 1975; Mangini and Pearlman, 1980; Wagor et al., 1980; Metin et al., 1988). Consequently, the visual cortex of mice is dominated by visual inputs coming from the contralateral eye and the term 'contralateral dominance' is used to describe this phenomenon as mentioned before.

\subsection{Ocular dominance as a model for brain plasticity}

Since the sixties of the last century, ocular dominance (the cortical response to visual stimulation of one or the other eye) has been a powerful method to study cortical processing and plasticity in the brain. It has been examined in different mammals including monkeys (Horton and Hocking, 1997), cats (Hubel and Wiesel, 1963; Hubel and Wiesel, 1970), ferrets (Issa et al., 1999) and rats (Domenici et al., 1992; Maffei et al., 1992; Fagiolini et al., 1994). However, presently the mouse visual cortex has become the standard model to investigate OD-plasticity because it shares both many similarities to the visual system of humans and there are versatile investigations possible due to the large number of available genetically modified knockouts (Dräger, 1978; Gordon and Stryker, 1996; Bartoletti et al., 2002; Lehmann and Löwel, 2008 Frenkel and Bear, 2004; Tagawa et al., 2005; Hofer et al., 2006) (for review see Espinosa and Stryker (2012) and Levelt and Hübener (2012)). 
Animals like carnivores and primates have a refined visual system which includes a much larger cortical region for visual processing and orientation. Unlike mice, they show orientation columns in the visual cortex, meaning that groups of neurons within a column perpendicular to the surface of the cortex have nearly identical receptive fields and similar response properties which is commonly referred to as 'columnar organization` (Hubel et al., 1976; Issa et al., 2000; Ohki and Reid, 2007; Van Hooser, 2007; DeFelipe et al., 2012).

Still, the overall organization of the mouse visual cortex remains relatively simple which renders it an excellent model system for combining and bringing theoretical models into closer compliance with biological reality and relevance (Blais et al., 2008; Ohki et al., 2005). In rodents like rats and mice, neural cells in the visual cortex are not organized in orientation columns and therefore exhibit a lower degree of spatial organization in comparison to primates or carnivores (Ohki et al., 2005; Van Hooser, 2007). This phenomenon is referred to as 'salt-and-pepper' organization (Kaschube, 2014; for review see: Espinosa and Stryker, 2012). For pyramidal cells located in the surface of mouse V1, it has been reported that neurons with similar visual response properties excite each other (Harris and Mrsic-Flogel, 2013; Ko et al., 2011; Li et al., 2013; Lien and Scanziani, 2013; Wertz et al., 2015) but the anatomical basis of this synaptic network is still vastly unknown.

Contradictory to the fact that axons and dendrites of all orientation selectivity's pass near each other with roughly equal chance; it was shown that pyramidal neurons of similar orientation selectivity are forming synapses with each other and neurons with similar orientation tuning form larger synapses (Lee et al., 2016). In addition to that it was postulated that neurons in layer 2/3 pyramidal neurons of the mouse visual cortex exhibit similar motion direction preferences, which were developing layer-specific functional modules. In most of the networks (about two thirds), the direction preference varied between the layers, whereas in about one-third of the networks, the layer modules were locked to the direction preference of the postsynaptic neuron (Wertz et al., 2016).

OD-plasticity in the visual cortex is a method to study how experience and deprivation of one eye may modify connections in the brain (Blais et al., 2008). The mammalian primary visual cortex is not fully mature at birth and not even at the time of eye opening, both anatomically and physiologically. Hence, the cortex still shows plasticity and continuously develops further during the first weeks of postnatal life (Hubel and Wiesel, 1963; Blakemore and Van Sluyters, 1975; Dobson and Teller, 1978; Fregnac and Imbert, 1978; Albus and Wolf, 1984; Boothe et 
al., 1985; Fagiolini et al., 1994). Input signals from the two eyes firstly converge in the primary visual cortex (Wiesel and Hubel, 1963), where competitive interactions of the synapses determine which eye will eventually dominate both, functionally and anatomically (Sugiyama et al., 2008). For example, juvenile mice show less visual acuity than adult mice, nevertheless they achieve adult-like visual acuity at around postnatal day 28 (Prusky et al., 2006). In the mouse visual cortex, neurons have been shown to be orientation as well as direction-selective (Dräger, 1975; Metin et al., 1988; Sohya et al., 2007; Niell and Stryker, 2008; Wang et al., 2010). Already before eye opening, retinal ganglion cells exhibit strong direction selectivity (Elstrott et al., 2008; Yonehara et al., 2009) which is not dependent on visual experience (Elstrott et al., 2008; Chen et al., 2009; Yonehara et al., 2009; Rochefort et al., 2011). However, ocular dominance and binocular vision are rudimentary in immature animals (Sherman and Spear, 1982; Fagiolini et al., 1994). The gradual development of these functional properties during subsequent postnatal periods depends critically on appropriate visual experience (Gianfranceschi et al., 2003). During this critical period early in life, visual neurons develop their adult functional properties in response to visual stimuli. Hence, an extensive anatomical reorganization of connections in the visual cortex takes place (Fagiolini et al., 1994). The ocular dominance of binocular neurons in the visual cortex is actively maintained by competition between synapses which are serving the two eyes (He et al., 2006). In the binocular part of the visual cortex, neurons respond to inputs from both eyes, but remain to be dominated by the contralateral eye as shown in rodents (Dräger, 1975, 1978). Early during the developmental phase, connections in the mammalian central nervous system proceed through a period (known as the critical period) in which they exhibit a high degree of plasticity (Wiesel and Hubel, 1963; Gordon and Stryker, 1996; Hensch, 2005). Both, anatomical and functional development, depend greatly on visual experiences during this early phase of plasticity. In the visual cortex, this critical period ends after approximately 5 years in humans, 12 weeks in kittens and around 35 days in mice (Gordon and Stryker, 1996). After this critical point of time in development, the capacity for experience-dependent changes in the brain is substantially reduced through several mechanisms which are not fully understood yet (Sawtell et al., 2003; Schwarzkopf et al., 2007; Lehmann and Löwel, 2008; Morishita and Hensch, 2008). It is assumed that plasticity mechanisms in the brain are different in juvenile and adult mice. During the critical period in juveniles, plasticity is thought to be a homeostatic process that depends mechanistically on synaptic scaling (Kaneko et al., 
2008; Mrsic-Flogel et al., 2007). In adult mice, it is thought to be mediated by a different mechanism that requires $\alpha$-calcium/calmodulin-dependent kinase II ( $\alpha$ CaMKII) auto phosphorylation (Ranson et al., 2012). Visual experience acts by modulating the level of neural activity within the visual pathway (Fagiolini et al., 1994). It also plays a crucial role in strengthening, remodeling and the elimination of synapses during development of the visual system (Shatz et al., 1990; Fagiolini et al., 1994). Although early studies on ocular dominance were performed with cats (Wiesel and Hubel, 1963; Hubel and Wiesel, 1970), the mouse visual cortex has now become a standard model to examine OD-plasticity (Frenkel and Bear, 2004; Tagawa et al., 2005; Hofer et al., 2006).

An extensively investigated model for neuronal plasticity in the brain is the so-called ocular dominance (OD) plasticity. This is accomplished by depriving the contralateral eye of mice of vision which can result in a change in the ocular dominance. More precisely, neurons in the binocular region of $\mathrm{V} 1$ change their responsiveness and get activated equally strong by stimulation of each eye (Dräger, 1975; Gordon and Stryker, 1996).

\subsection{Monocular deprivation}

Experience-dependent cortical plasticity was already studied in the classic experiments by Wiesel and Hubel $(1963,1970)$, where a rapid ocular dominance shift in kittens was achieved by closing one eye (Mioche and Singer, 1989; Law and Cooper, 1994; Mitchell and Sengpiel, 2009). The scientists Wiesel and Hubel (1963) were the first ones performing a detailed investigation of a critical period at the neuronal level in the visual cortex of cats.

The critical period is commonly referred to as a stage early in development during which the nervous system is especially sensitive to certain environmental stimuli. If an appropriate stimulus like visual input is not perceived until the end of the critical period, it is difficult or even impossible to develop these specific functions later in life (Hensch, 20014). Vision and other senses that are essential to an organism's survival are especially likely to develop during critical periods. Occluding one eye of vision (monocular deprivation, MD) in kittens during a period of one to four months during early postnatal life resulted in a drastically changed OD in their visual cortex. Electrophysiological recordings showed that deprived eye stimulation became less effective in driving cortical cells to respond, whereas the open eye gained influence in activating cortical neurons. According to this, early MD produced an OD- 
shift towards the open eye and hence OD-plasticity (Wiesel and Hubel, 1963). These observations started OD-plasticity to be a well-established model for neuronal plasticity and since then MD is a widely used model to study experience-dependent cortical plasticity.

Up to now there are two different models for plasticity in the visual cortex which can be altered through monocular deprivation (MD). One model is for "sensory learning" and claims that through MD cortical neurons lose their responsiveness to the closed eye in favor of the open eye (Gordon and Stryker, 1996). According to Prusky et al. (2006), there is a second sensory "learning" paradigm which is dependent on the cortex. They state that monocularly deprived mice gain visual acuity and contrast sensitivity in the open eye due to possible synaptic changes in the visual cortex. In some cases, ocular dominance can be reversed by monocular deprivation as described in the following paragraph.

Early monocular occlusion permanently reduces visual acuity (amblyopia) of the deprived eye (Daw et al., 1992; Daw, 1998; Prusky and Douglas, 2003). Unbalanced sensory experience in the eyes leads to a rapid shift of neuronal responses in favor of the open eye (ocular dominance) accompanied by a rapid increase of dendritic spines and later also by a rewiring of thalamo-cortical afferents (Wiesel and Hubel, 1963; Antonini et al., 1999; Hensch, 2005; Sugiyama et al., 2008). Monocular deprivation causes cortical neurons to reduce or lose their responsiveness to the closed eye as long as the brain shows plasticity. This process is dependent on the activity in the deprived eye (Blakemore, 1976; Bear and Rittenhouse, 1999; Rittenhouse et al., 2006) and not simply due to the fact of decreased light intensity for example. In mice, the peak of the critical period for ocular dominance plasticity lies between postnatal days (P) 25 and 30 (Gordon and Stryker, 1996). During this critical period, four days of monocular deprivation are already sufficient to drive binocular cortical neurons equally responsive to both eyes (Gordon and Stryker, 1996; Lehmann and Löwel, 2008). Indeed, brief monocular deprivation does induce a rapid shift in the ocular dominance of binocular neurons in the juvenile rodent visual cortex, whereas it is not effective in adult animals when they were reared in standard cages (Frenkel and Bear, 2004; Lehmann and Löwel, 2008). These effects of monocular deprivation can be blocked by antagonists of the N-Methyl-D-aspartate (NMDA) subtype of the glutamate receptor (Bear et al., 1990) and occlude long-term depression induced by low frequency stimulation (Heynen et al., 2003). Nevertheless, a study conducted by Lehmann and Löwel (2008) showed that ocular dominance plasticity can also be observed in young adult mice, although a longer deprivation period was needed (6-7 days) 
(Sawtell et al., 2003; Tagawa et al., 2005; Hofer et al., 2006; Sato and Stryker, 2008). The researchers concluded that there is an age-dependence of both, OD-plasticity in the visual cortex and the increase of visual acuity after monocular deprivation. In mice younger than 110 days of age (at this time they are not fully mature), monocular deprivation induced a significant ocular dominance shift towards the open eye. This shift of ocular dominance was absent in animals aged $110-230$ days when housed in standard conditions, even after longer deprivation times up to 14 days (Lehmann and Löwel, 2008). Regarding the visual acuity in mice with monocular deprivation from 25 up to 230 days of age, visual acuity significantly increases in all tested age groups. However, in animals older than P 110, this interocular plasticity also declines with age. The results of the study carried out by Lehmann and Löwel showed that ocular dominance plasticity in mice was inducible after monocular deprivation during the critical period (P 20 - P 35) in early postnatal development, already declined in young adults (P 95) and was not inducible in fully mature animals raised in standard cages ( $P$ 130 and P 215) (Lehmann and Löwel, 2008). These important findings illustrate that ocular dominance plasticity in mice is certainly age-dependent. However, this cortical plasticity does not end suddenly at one point of time in development but rather declines gradually. These results show that mice exhibit a critical period for OD-plasticity in early life ( $20-35$ ) and therefore do not basically differ from cats and monkeys (and probably also humans) in visual cortical plasticity. This is a very powerful and strictly essential argument for the use of mice as model system for disorders of the human visual system like amblyopia (Lehmann and Löwel, 2008). 


\subsection{Critical period for OD-plasticity in mice}

\subsubsection{Opening of the critical period for OD-plasticity}

Research about the mechanisms regulating the beginning and the end of critical periods has been going on for many years (for review see: Berardi et al., 2000). Early in the developmental state excitation appears to dominate cortical circuits, but much research was done to support the view of a central role for late-developing excitation/inhibition (E/I) circuit balance in the initiation of sensitive periods (Bavelier et a., 2010) and the timing of the critical period for ODplasticity. It was shown that the development of inhibitory circuits in the cortex and the function of inhibitory neurons are crucial for the opening of the critical period (for review see: Hensch, 2005).

There are several mechanisms discussed which might open and close the critical period for OD-plasticity.

The development of GABAergic stimulation and a minimal level of GABAergic inhibition is decisive for the onset of the critical period which was shown in GAD65 (GABA-synthesizing enzyme glutamic acid decarboxylase) knockout (KO) mice. These KO mice exhibit reduced GABA synthesis due to the inactivation of GAD65 (Hensch, 1998) and completely lack ODplasticity after 4 days of monocular deprivation during the critical period (P $25-27$ ). However, by enhancing inhibition in GAD65 KO mice with diazepam treatment, which is a GABAA receptor agonist (positive allosteric GABAA receptor modulator), the CP for OD-plasticity opens (Hensch, 1998; Fagiolini \& Hensch, 2000). Similarly, to that, in young WT mice (P 15), treatment with diazepam could induce an earlier onset of the CP that was alike in duration to the normal critical period (Fagiolini and Hensch, 2000). This finding supports the view that a transient increase in GABAergic transmission is required to open the critical period for ODplasticity.

Likewise, it was found that GABAergic transmission acting via the $\alpha 1$ subunit of GABAA receptors is fundamental for the opening of the critical period. The $\alpha 1$ subunit of GABAA receptors is enriched in inhibitory synapses which are formed by fast-spiking parvalbumin (PV) positive interneurons (Klausberger et al., 2002). Knock-in mice with insensitive GABAA receptor subunits were treated with diazepam and showed that only the mutant $\alpha 2$ and $\alpha 3$ GABAA receptor subunits could initiate a precocious onset of the critical period, similar to 
that of WT mice treated with diazepam (Fagiolini et al., 2004). These findings suggest that inhibitory neurons like the PV basket cells and hence GABAergic transmission play an important role in opening the critical period for OD-plasticity in mice.

In fact, while the maturation of inhibitory PV cells was slowed down by using mutant mice exhibiting weakened circadian gene expression, the onset of critical period plasticity was delayed but could be restored by direct enhancement of GABAergic transmission with diazepam (Kobayashi et al., 2015).

The maturation of inhibitory circuits can also be regulated by factors that are involved in the opening of the critical period for OD-plasticity. Transgenic mice overexpressing the brainderived neurotrophic factor BDNF in pyramidal neurons in the visual cortex during postnatal development showed an earlier maturation of inhibitory neurons and GABAergic innervation with accelerated inhibition (Huang et al., 1999). Hence, overexpression of BDNF resulted in an earlier onset of the CP for OD-plasticity which in the course also terminated precociously (OD-plasticity was blocked after 4 days of MD at P 28 measured with VEP recordings in the visual cortex). This is in line with the results of Hanover et al. (1999), where mice overexpressing BDNF showed a precocious peak of the CP for OD-plasticity at P $21-23$ and an earlier closure of it already before P 27.

Other growth factors like the insulin-like growth factor called IGF-1 can likewise accelerate the maturation of circuitry and inhibitory innervations (Tropea et al., 2006; Ciucci et al., 2007). IGF-1 is a peptide that is implicated in prenatal and postnatal phases of brain development such as neurogenesis, neuronal differentiation and experience-dependent plasticity (MayaVetencourt et al., 2012).

Isoforms of the neuronal cell adhesion molecule (NCAM) carrying the polysialic acid (PSA) have emerged as attractive candidates for promoting plasticity in the brain as well. The PSA chain of NCAM is negatively charged and therefore thought to be a spacer between cells, reducing adhesion between them and allowing dynamic changes in membrane contacts. During development, the expression level of PSA-NCAM is high and it appears to play a role in regulating cell shapes, growth or migration (for review see: Gascon et al., 2007). Additionally, PSA-NCAM has been implicated in the opening of the critical period (Di Cristo et al., 2007).

Moreover, the opening of the critical period for OD-plasticity does not only depend on internal regulation factors, but also largely on the individual visual experience of the animal. 
Chronic dark rearing from birth and hence a lack of visual experience during the critical period, results in a delayed onset of the critical period (Fagiolini et al., 1994). Additionally, dark rearing prevents the maturation of AMPA receptor silent synapses in layer 2/3 pyramidal neurons that are normally decreasing during aging in a normal visual environment (Funahashi et al., 2013). After dark rearing, the fraction of silent synapses remained considerably high in mice even until adulthood. This suggests that the conversion from silent to fully functional synapses depends on age and experience and might be one of the synaptic mechanisms underlying experience-dependent refinements of visual cortical circuits (Funahashi et al., 2013).

Furthermore, at the time between eye opening and the end of the critical period, GABAergic inputs into layer 2/3 pyramidal neurons largely increase. This developmental GABAergic input is however prevented when animals are reared in darkness from birth but not when exposed to light before visual deprivation starts (Morales et al., 2002). Therefore, visual sensory experience might play an important role in the maturation of intracortical GABAergic circuits. Dark rearing was also shown to reduce the brain-derived neurotrophic factor (BDNF) levels and hence, delaying the critical period (Zafra et al., 1990). However, when cortical BDNF levels were increased in dark reared mice, this resulted in a normal critical period for OD-plasticity (Gianfranceschi et al., 2003). Together, these observations suggest that the effects of dark rearing on plasticity involve the maturation of the inhibitory circuit at least partly.

In fact, it was found that a very short period of darkness /dark exposure (for 10 - 14 days) can restore OD-plasticity in juvenile rats (He et al., 2006) as well as in mice which were already beyond their sensitive phase for OD-plasticity (> P 110), which exhibited a juvenile-like ODshift after only 4 days of MD (Stodieck et al., 2014).

Studying OD-plasticity in the mouse VC has turned out to be essential for the understanding of critical period timing. As mentioned above, studies using both transgenic mice and pharmacological approaches have helped to identify possible molecular candidates and mechanisms that are involved in controlling the opening of the critical period for ODplasticity. While there is little doubt that the maturation of inhibitory circuits is important for the onset of the critical period (Fagiolini and Hensch 2000; Fagiolini et al., 2004; Hensch et al., 1998), it is not fully understood how inhibitory cells like PV positive neurons exactly take part in OD-plasticity. This is also partly due to controversial findings about the eye-specific visual 
cortical responses changes after monocular deprivation (Gandhi et al., 2008, Kameyama et al., 2010, Mainardi et al., 2009, Yazaki-Sugiyama et al., 2009).

A clear picture of the underlying mechanisms that are responsible for the closure of the critical period for OD-plasticity was also lacking. Several factors limiting OD-plasticity in adult animals have been identified, but it was not clear which of these factors are involved in terminating the critical period and how they are interacting.

\subsubsection{Closing the critical period for OD-plasticity}

Recent research has identified the homeoprotein Otx2 as a possible molecular 'messenger' that is necessary for experience-driven visual plasticity during the CP (Sugiyama et al., 2009). In the absence of Otx2, the CP is delayed potentially by impairing the development of parvalbumin expressing (PV) GABAergic interneurons, but can be rescued by intracortical infusion of Otx2 (Sugiyama et al., 2008).

Furthermore, Otx2 appears to enter PV positive interneurons through an interaction with dense aggregates of the extracellular matrix (ECM), known as perineuronal nets (PNNs), which are composed of chondroitin sulfate proteoglycans (CSPGs) and other extracellular matrix components that enwrap these neurons. PNNs might play an important role in plasticity by modulating PV cell function (Berardi et al., 2004; Sugiyama et al., 2009). Their progressive developmental increase is thought to contribute to the closure of critical periods since the expression in the VC increases during development and coincidences with the closure of the CP for OD-plasticity (Pizzorusso et al., 2002).

Another postulated factor limiting OD-plasticity during the CP (and adulthood) is the paired Ig-like receptor $\mathrm{B}$ (PirB), a histocompatibility complex class I $(\mathrm{MHCl})$ receptor expressed in cortical pyramidal neurons (and other regions in the CNS) (Syken et al., 2006). It was shown that 3 days of MD in adult (P 90) PirB KO mice induced a large OD-shift mediated by increased open eye responses, increased spine density and decreased spine motility on layer 5 cortical pyramidal neurons which might explain enhanced sensory-driven plasticity in the VC of PirB KO mice (Djurisic et al., 2013). These results suggest that PirB stabilizes neural circuits (Syken et al., 2006), whereas its deactivation could be a potential target for regeneration therapies (Atwal et al., 2008). Like mice lacking functional PirB, adult mice (P 120) lacking the Nogo 
receptor (NgR) or its ligands (Nogo- A/B) exhibit OD-plasticity well beyond the CP after 4 days of MD (McGee et al., 2005).

Albeit structural brakes like PNNs (Pizzorusso et al., 2002), Nogo receptors (McGee et al., 2005), or PirB (Syken et al., 2006) can limit plasticity by altering local connectivity, functional brakes like lynx1 can stop plasticity by changing the neurotransmission between synapses that have been formed to facilitate plasticity (Nabel \& Morishita, 2015). Lynx1 is an endogenous prototoxin, which acts by binding to the nicotinic acetylcholine receptor ( $\mathrm{nAChR}$ ) and therefore reduces its activation (Morishita et al., 2010). Increases in lynx1 expression correlate well with the closure of the CP in mouse VC and limits functional plasticity into a late state (Morishita et al., 2010), while the removal of it allows the CP to remain open until nAChR signaling is actively blocked (Miwa et al., 1999). Thus, lynx1 expression preserves the stability of mature cortical networks in the presence of cholinergic innervation (Morishita et al., 2010). Although there are many molecular candidates discussed in regulating the onset and the closure of the CP for OD-plasticity during the last years, however, it could not be directly shown in experiments yet what controls the reassembling of synapses during the CP where neural networks are improved to gain their optimal functional properties.

It has been proposed that synaptic connections are strengthened during development by correlated pre- and postsynaptic activity (Hebb, 1949; Stentt, 1973). A likely mechanism for this process would be $\mathrm{N}$-methyl-D-aspartate (NMDA) receptor-dependent long-term potentiation (LTP) (Collingridge and Singer, 1990; Kandel et al., 1992). The molecular mechanisms of LTP in the immature brain are thought to differ from those mechanisms that underlie LTP in the adult brain (Yasuda et al., 2003). There is some evidence that LTP is important for the development of cortical circuitry and that the molecular mechanisms of LTP change during the developmental process (Esteban et al., 2003; Jensen et al., 2003; Kirkwood et al., 1997; Yasuda et al., 2003). These changes are thought to include a switch from LTP that is independent of calmodulin-dependent protein kinase II (CaMKII)- activation, which expression is low during early development, to CaMKII-dependent LTP later in life (Kirkwood et al., 1997; Yasuda et al., 2003). For early postnatal development, cyclic adenosine monophosphate (CAMP) -dependent protein kinase is proposed to be required for LTP (Yasuda et al., 2003). However, the evidence that LTP is involved in normal development still remains difficult to outline (see review: Malenka and Bear, 2004). Nevertheless, it was 
reported that the point of time during which LTP can be induced matches the CP. Furthermore, a loss of sensitivity to LTP with age is proposed to be accompanied by a decrease in NMDA receptor-mediated synaptic currents (Crair and Malenka, 1995).

It is widely accepted that a major mechanism for the expression of LTP involves increasing the numbers of AMPA receptors in the plasma membrane at synapses via activity-dependent changes in AMPA receptor trafficking (Bredt and Nicoll, 2003; Malenka and Nicoll, 1999; Malinow and Malenka, 2002; Song and Huganir, 2002; for review see: Malenka and Bear, 2004).

It is thought that synapses which essentially contain NMDA but no AMPA receptors (therefore AMPA receptor silent synapses) can serve as powerful plasticity substrates during early cortical network optimization (Isaac et al., 95; Isaac et al., 1997; Liao et al., 1995). It is suggested that these silent synapses are immature and develop through experience from excitatory synapses containing NMDAR to mature synapses containing both, NMDA and AMPA receptors and therefore acquire their full functional properties. This sensory experience dependent maturation of silent synapses is thought to cause the termination of critical periods, which was recently proven experimentally (Huang et al., 2015). This challenged the prevailing view that increased local inhibition and the expression of plasticity brakes like PNNs, Nogo receptors, lynx1 or PirB are terminating critical periods (Pizzorusso et al., 2002; Mataga et al., 2002; McGee et al., 2005; Syken et al., 2006).

One molecular candidate governing plasticity on a cellular level during the CP is a scaffold protein found abundantly in the postsynaptic density (PSD) named PSD-95, whose expression in the VC increases during eye opening and therefore with the onset of visual experience (Yoshii et al., 2003). In hippocampal neurons it was already shown that PSD-95 promotes the maturation of AMPA receptor-silent excitatory synapses (Ashby et al., 2011; Funahashi et al., 2013) and that PSD-95 is required for the proper stabilization of synapses (Stein et al., 2003; Béique et al., 2006; Ehrlich et al., 2007).

We hypothesized that the maturation of silent synapses is experience-dependent and results in the strengthening of excitatory synapses which control the stabilization and refinement of networks during critical periods and the progressive decrease of silent synapses leads to the closure of critical periods (Huang et al., 2015). 


\subsection{DLG MAGUKS}

Chemical synapses pass information directionally from a presynaptic to a postsynaptic cell. The presynaptic terminal or also referred to as synaptic bouton is a specialized area within the axon of the presynaptic cell that contains neurotransmitters. These neurotransmitters are stored in synaptic vesicles attached to the presynaptic plasma membrane at the active zones from where they can be released into the synaptic cleft. Immediately opposing the presynaptic cell is the postsynaptic cell where neurotransmitter receptors are incorporated into the membrane. Immediately behind the postsynaptic membrane is an elaborate electron-dense protein-enriched complex of interlinked proteins called the postsynaptic density (PSD), whose protein composition and morphology changes with neural activity (Kim and Sheng, 2009).

The PSD serves several different functions in the brain including facilitation of membrane receptor anchoring in dendritic spines, the trafficking and modulation of molecules and proteins, and ion channels like AMPA and NMDA receptors (Feng and Zhang, 2009; Kim and Sheng, 2004; Vallejo et al., 2016).

The dynamic composition of the PSD with its different components that are continuously reorganized and exchanged (Blanpied et al., 2008; Kerr et al., 2012; Kuriu et al., 2006; Sturgill et al., 2009) can control the amplitude of postsynaptic currents and therefore regulate both the strength and plasticity of excitatory neurotransmission (Vallejo et al., 2016).

Proteins in the PSD are involved in anchoring and trafficking of glutamate receptors and modulate the activity of them. This allows proper modifications in synaptic strength during synaptic plasticity.

The PSD contains one superfamily of scaffold proteins accumulated named the disc-large (DLG) of membrane-associated guanylate kinases (MAGUK). This family includes the postsynaptic density protein (PSD) -95 (SAP 90), PSD-93 (Chapsyn - 110), the synapse associated protein (SAP) 97 and SAP-102. All members of this family share a similar structure of three domains: First, the inclusion of PDZ, which is an acronym combining the first letters of three proteins: PSD95, Drosophila disc large tumor suppressor (Dlg1), and Zonula occludens-1 protein (zo-1), second the Src-homology-3 (SH3), and third the catalytically inactive guanylate kinase GK domain (Cho et al., 1992; Craven and Bredt, 1998; Kim and Sheng, 2004). Despite of their shared similar structure, DLG-MAGUKs are different concerning the 
amino acid sequence of their $\mathrm{N}$-terminal domains, which exhibit unique roles in regulating synaptic functions (Schlüter et al, 2006), and the structural organization of the PSD which is important for synapse development and plasticity (Irie et al., 1997; Kornau et al., 1995; Kim et al., 1997).

Besides SAP-97, which directly binds to AMPA receptors at their GluA1 subunits (Leonard et al, 1998), all other DLG-MAGUKs do not directly bind to AMPA receptors. Therefore, an intermediary protein must fulfill this function. It was shown that amongst others, the transmembrane protein stargazin, which is the most representative member of the transmembrane AMPA receptor regulatory proteins (TARPs) (Nicoll et al., 2006; Sager et al., 2009), might likely serve this role (Chen et al., 2000; Schnell et al., 2002; Tomita et al., 2003). TARPs directly bind to AMPA receptors and to the first two PDZ domains of the PSD- MAGUKs (Chen et al., 2000). In contrast to that, all DLG-MAGUKs directly interact with the GluN2A and GluN2B NMDAR subunits (Kornau et al, 1995; Niethammer et al, 1996). The MAGUK proteins are thought to be fundamental players in regulating the trafficking of glutamate receptors and orchestrating the functional organization of the PSD due to their ability to interact with both, AMPARs and NMDARs.

A complete deletion of the protein SAP-97 (SAP-97 KO) is lethal in mice, which also demonstrates the importance of the MAGUK protein. However, if SAP-97 was only conditionally knocked out, this was shown to cause no major deficits in glutamatergic transmission or long-term potentiation (Howard et al, 2010). Overexpression of SAP-97 on the other hand enhances AMPAR and NMDAR currents (Rumbaugh et al, 2003; Howard et al, 2010), or had no impact on postsynaptic AMPARs (Schnell et al, 2002; Ehrlich and Malinow, 2004; Schlüter et al, 2006).

If SAP-102 is deleted in immature synapses, AMPAR neurotransmission is decreased (Elias et al, 2006) whereas in the mature state, basal neurotransmission is normal but NMDARdependent LTP is enhanced (Cuthbert et al, 2007). Importantly, SAP-102 can counteract for PSD-95 deletion and the loss of synaptic AMPARs (Elias et al, 2006; Bonnet et al, 2013). The last two MAGUK family members, PSD-93 and PSD-95, will be introduced in the following sections.

It is speculated that the DLG-MAGUK proteins play different roles in regulating neurotransmission and synaptic maturation at different developmental stages since they exhibit varying expression profiles. 
SAP 97 is already expressed about $50 \%$ at birth and further increases from P 7 until it reaches adult levels at P 14 (Krüger, 2010; Wang et al., 2006).

SAP-102 reaches adult levels already at $P 10$ which further underlines its important role in regulating AMPA receptors at synapses which are still immature (Sans et al., 2000; Krüger et al., 2010).

Both, PSD-93 and PSD-95 show very similar developmental profiles since they are poorly expressed at birth and then steeply increase in their expression levels at around P 12, which coincidences with eye opening and therefore also with visual experience. Both proteins reach adult levels at around P 35 (Sans et al., 2000; Krüger et al., 2010) and share similar N-terminal palmitoylation sites. These are important for linking with membrane lipids and stability at the postsynaptic membrane (Sturgill et al., 2009), for the localization of the proteins and for the clustering of receptors (Craven et al., 1999; Topinka and Bredt, 1998; El-Husseini et al., 2002).

\subsubsection{The postsynaptic density protein-95 (PSD-95)}

One of the most abundant proteins located in the postsynaptic density is PSD-95. It is the best studied member of the DLG-MAGUK family with $95 \mathrm{kDa}$ in size and in humans encoded by the disk large homolog gene 4 (DLG4) (Cho et a., 1992; Stathakis et al., 1997).

PSD-95 is not only localized in excitatory synapses of the postsynaptic density in the brain but also in rod and cone photoreceptor terminals in the retina, which was studied in different mammals including rats, monkeys and tree shrews by using immunocytochemical methods (Koulen et al., 1998). PSD-95 is clustered in the postsynaptic terminals of bipolar cell ribbon synapses and thought to be expressed in synapses of amacrine and ganglion cells which receive inputs from bipolar cells (Koulen et al., 1998). 


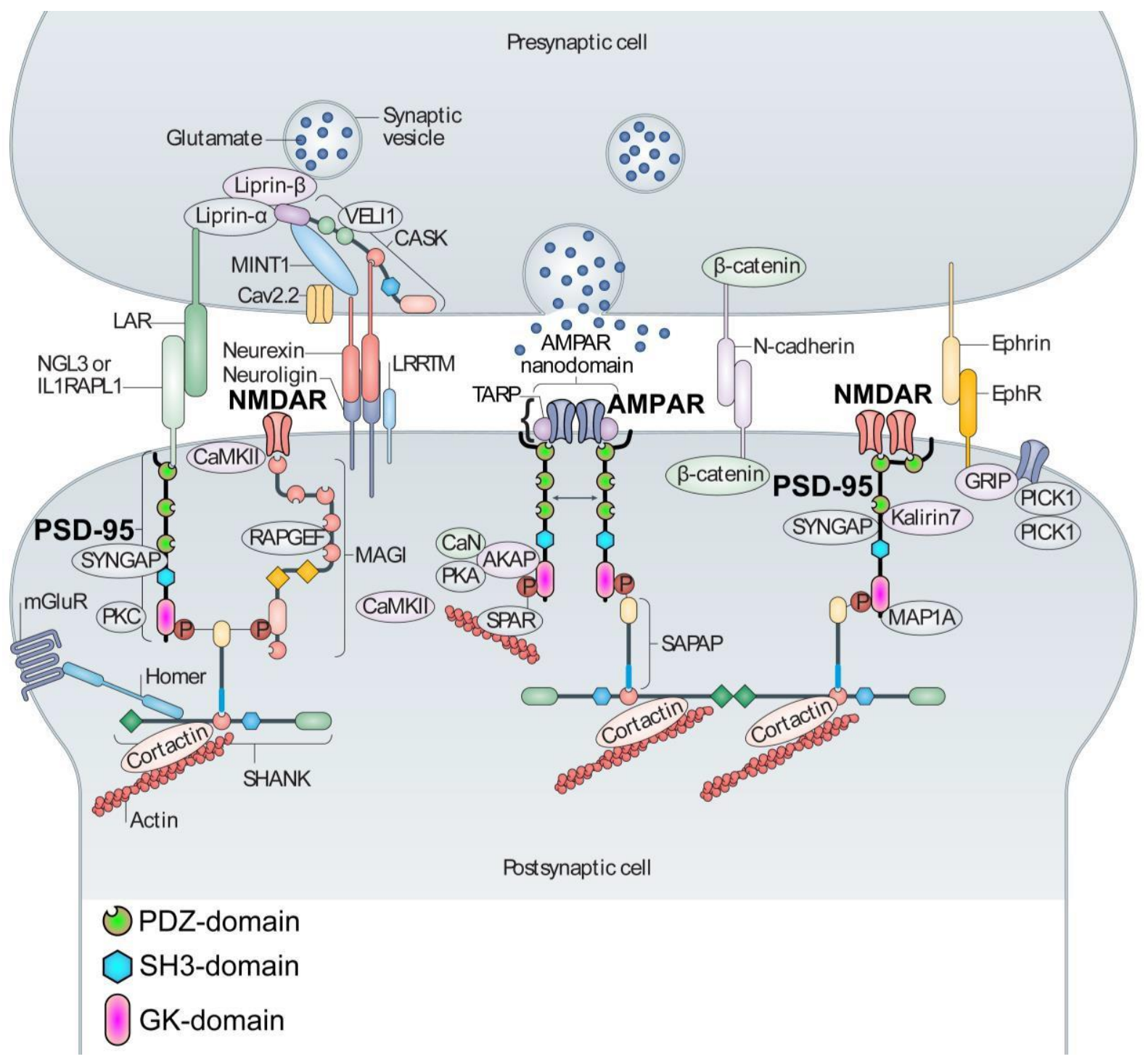

Figure 3: Molecular organization of glutamatergic synapses (Figure modified from Zhu et al., 2016). Illustrated are molecules which are directly linked to PSD-95 including NMDARs (red) and neuroligins (purple) as well as molecules which are indirectly connected to PSD-95 via TARPs (stargazin) and AMPARs (blue). These various molecules regulate synapse function, morphology, trafficking, localization of adhesion molecules, neurotransmitter receptors, and additionally play an important role in synaptic plasticity. PSD-95 consists of three different domains including the catalytically inactive guanylate kinase domain (GK), the Src-homology-3 (SH3) and the three PDZ domains.

PSD-95 is a scaffolding protein and essential for the direct and indirect anchoring of receptors (e.g. AMPARs, NMDARs, mGluRs), ion channels (e.g. $\mathrm{K}^{+}$channels) and postsynaptic adhesion molecules like neuroligins (Sheng and Sala, 2001; Keith and El-Husseini, 2008) (Figure 3). PSD95 exhibits a variety of different functions including the regulation of dendritic spine maturation (El-Husseini et al., 2000), the stabilization of dendritic branches and outgrowth 
(Charych et al., 2006), the reorganization of the presynaptic terminal (El-Husseini et al., 2000), modulation of trafficking of receptors during development (Van Zundert et al., 2004) and the induction of LTP (Zhao et al., 2013; Ehrlich and Malinow, 2004). Sufficient amounts of PSD-95 are especially important for activity-dependent synapse stabilization after the initial phases of LTP induction, which mainly depends on the incorporation of AMPA receptors into the membrane of synapses (Ehrlich et al., 2007). Palmitoylation of PSD-95 can regulate the synaptic intensity by controlling the fraction of AMPA receptors at the postsynaptic membrane (El-Husseini et al., 2002; Chen et al., 2015). Hence, the strength of excitatory synaptic transmission can be regulated by the trafficking of AMPARs to or from synapses. The amount of PSD-95 in the PSD is directly related to the strength of AMPAR neurotransmission and therefore responsible for the strength of AMPAR mediated excitatory postsynaptic currents (EPSCs). However, PSD-95 is not static at the synapse but rather transported dynamically to and from the postsynaptic membrane which is thought to be regulated by synaptic activity (Vallejo et al., 2016). A change in the expression of PSD-95 has a direct effect on both the distribution and the number of AMPARs in the postsynaptic membrane (Béique et al., 2006; DeGiorgis et al., 2006; Levy et al., 2015). It indirectly stabilizes AMPARs through the protein stargazin (Chen et al., 2000; Chetkovich et al., 2002; El-Husseini et al., 2002; Schnell et al., 2002; Dakoji et al., 2003; Tomita et al., 2003; Fukata et al., 2005; Tomita et al., 2005; Bats et al., 2007). Manipulating PSD-95 expression in hippocampal neurons revealed that it can enhance synaptic clustering and AMPARs function by regulating the maturation of excitatory synapses, when it is overexpressed (El-Husseini et al., 2000; Tomita et al., 2001). However, an acute knockdown as well as a knockout of PSD-95 decreases AMPAR-mediated synaptic transmission in mice (Migaud et al., 1998; Carlisle et al., 2008) and leads to a removal of all AMPARs in half of the excitatory synapses (Migaud et al., 1998; Béique et al., 2006; Elias et al., 2006; Fitzjohn et al., 2006; Ehrlich et al., 2007). Due to these findings, one can conclude that PSD-95 stabilizes the receptors in the synapse (Opazo et al., 2012) and plays a major role in mediating AMPAR targeting especially at mature synapses (Elias et al., 2006). Additionally, it is largely involved in regulating synaptic plasticity (Migaud et al., 1998; Ehrlich and Malinow, 2004; Yao et al., 2004; Béïque et al., 2006; Elias et al., 2006; Keith and El-Husseini, 2008; Sun and Turrigiano, 2011; Huang et al., 2015). A lack of PSD-95 also leads to the weakening of synapses and impaired long-term depression (LTD) (Chen et al., 2015; Nelson et al., 2013; Xu 
et al., 2008; Opazo et al., 2011; Colledge et al., 2003; Sturgill et al., 2009; Bhattacharyya et al., 2009).

Nevertheless, when PSD-95 is overexpressed in mice, LTP is blocked and LTD is facilitated (Béique and Andrade, 2003; Stein et al., 2003), whereas LTP is enhanced and LTD is blocked when PSD-95 is absent (Migaud et al., 1998). These data suggest that PSD-95 is not directly recruiting AMPARs to the synapse but rather acts as a mediator of postsynaptic signaling cascades downstream of NMDARs (Migaud et al., 1998; Tomita et al., 2001; Yao et al., 2004; Carlisle et al., 2008; Xu et al., 2008). This is also supported by the findings that PSD-95 is directly interacting with NMDARs but has no effects (Kornau et al., 1995), or little effects on NMDAR mediated EPSCs (Levy et al., 2015). Hence, it exhibits a specialized role in determining the number of AMPARs at excitatory synapses (Elias and Nicoll, 2007), which in turn plays a critical role in LTP and LTD. However, the facilitation of LTP is not based on changes in NMDAR function, it is rather attributed to a higher number of AMPA receptor silent synapses (Carlisle et al., 2008). AMPA receptor silent synapses are special synapses which are most abundant during the critical period and only contain NMDARs but no fully functional AMPARs (Malenka and Nicoll, 1997, 1999; Malinow et al., 2000; Tomita et al., 2001; Béïque et al., 2006). In AMPA-silent synapses, neurotransmitter release fails to elicit EPSCs when the synapse is at resting membrane potential. For that reason, they are considered to be not fully functional or rather silent (Isaac et al., 1995; Liao et al., 1995). These silent synapses can be characterized by measuring their miniature EPSC frequencies (mEPSC), which are postsynaptic responses to spontaneously released single vesicles, or by using minimal stimulation assays (Graziane and Dong, 2016). When the amplitude of AMPA mEPSC is changing, this may be due to either changes in the number of AMPAR or due to changes in the conductance of single channels at existing synapses. If the frequency of AMPAR mESPC is changed, this depicts changes in the presynaptic release of glutamate. A large portion of silent synapses results in decreased mESPC frequency, whereas a small portion results in increased mEPSC frequency. Silent synapses can become unsilenced by the insertion of AMPAR into the synaptic membrane, which can also move into dendritic spines by LTP induction. The PDZ domains of PSD-95 play a major role in regulating this insertion of AMPAR (Craven and Bredt, 1998; Tomita et al., 2001; Schnell et al., 2002; Dakoji et al., 2003; Ehrlich and Malinow, 2004; Schlüter et al., 2006; Bhattacharyya et al., 2009). If PSD-95 expression is overexpressed in hippocampal pyramidal 
cells, one can see a lower failure rate of AMPAR transmission compared to that of normal WT cells, indicating a decrease in the number of silent synapses (Stein et al., 2003).

Since most of the studies about PSD-95 were conducted in the hippocampus, there was little knowledge about the function of PSD-95 in the visual cortex. A study published in 2011 by Sun and Turrigiano showed that PSD-95 is involved in synaptic plasticity. They measured the fluorescence intensity of PSD-95 puncta during synaptic scaling in the visual cortex of rats and showed that the abundance of PSD-95 is bidirectionally regulated by neuronal activity. It was postulated that PSD-95 functions as an important synaptic organizer by employing proteinprotein interactions to mediate homeostatic accumulation and the loss of synaptic AMPAR. During development of the brain, AMPA receptor silent synapses are abundant (Isaac et al., 1997; Isaac et al., 1995; Liao et al., 1995; Rumpel et al., 1998) but the number is gradually decreasing with age. The number of AMPA silent synapses is elevated in the hippocampus of PSD-95 KO mice (Béïque et al., 2006; Levy et al., 2015), and recently it was shown that the conversion from silent to mature synapses is also impaired in the visual cortex of PSD-95 KO mice (Huang et al., 2015). Developmental refinements for OD-plasticity occur during the CP (Liu et al., 2008) in synapses of excitatory projections from layer 4 to layer $2 / 3$ pyramidal cells in the visual cortex. Recently, it was shown that the portion of silent synapses among the number of total synapses in WT mice was about $55 \%$ before eye opening and dropped to 25 $\%$ during the critical period and further decreased to only $5 \%$ in young adult mice beyond the CP for OD-plasticity (Huang et al., 2015). This indicates that before eye opening, about $50 \%$ of the synapses are AMPAR silent but this portion decreases with ageing and in an experiencedependent maturation process after eye opening (Huang et al., 2015). In PSD-95 KO mice, the number of AMPA silent synapses was similar to that of WT mice (55\%) before eye opening but remained high throughout the development and did not even decline after the CP for ODplasticity. These data indicate that PSD-95 is not only responsible for anchoring AMPARs but also absolutely required for the experience-dependent maturation of excitatory synapses onto pyramidal cells in the visual cortex after eye opening. Additionally, it was shown that the ratio of AMPAR to NMDAR of pyramidal neurons in the visual cortex was increasing in WT mice during the developmental process, whereas this was not the case in PSD-95 KO mice. Here, the AMPAR to NMDAR ratio remained low even after the critical period. This suggests that a juvenile-like AMPAR/NMDAR ratio is preserved in mice without PSD-95 and much more 
excitatory synapses onto pyramidal neurons remain in an immature state compared to that of WT mice (Huang et al., 2015).

As already described before, inhibition is one major player in contributing to the end of the CP for OD-plasticity (Fagiolini et al., 2004; Hensch et al., 1998; Sugiyama et al., 2008; Jiang et al., 2010). Likewise, reducing the inhibitory tone in adult rodents can also restore ODplasticity when it would be usually not present anymore after the CP (Harauzov et al., 2010; Morishita et al., 2010; Maya Vetencourt et al., 2008; Baroncelli et al., 2011). Hence, it could have been possible that the preserved juvenile-like state in PSD-95 KO mice is due to a reduction of the inhibitory tone. Key marker proteins of inhibitory synapses in the postsynaptic density including gephyrin, which is a major scaffolding protein at inhibitory synapses, and the $G_{A B A}$ receptor subunit $\alpha 1$ which is enriched at inhibitory synapses of parvalbumin positive interneurons, are thought to be analogous in the function to that of PSD-95 at glutamatergic synapses (Giesemann et al., 2003; Ehrensperger et al., 2007). The occurrence of both proteins was normal in adult PSD-95 KO mice, suggesting that the inhibitory synaptic transmission is unaltered in these mice. Additionally, the inhibitory tone in pyramidal neurons in the layer 4 to layers $2 / 3$ circuit was normal and the GABA/NMDA receptor ratio increased by about $80 \%$ from the critical period to young adult mice, which is in accordance to the typical developmental increase of the GABAergic tone during development. In summary, it was shown that PSD-95 does neither effect excitatory synaptic transmission onto parvalbumin positive interneurons, nor the GABAergic tone from these interneurons onto pyramidal neurons (Huang et al., 2015).

Since a global knockout of PSD-95 in mice does not allow a proper relation of cause and effect in the visual cortex, the causality between PSD-95 manipulations, pyramidal neuron synaptic maturation and the closure of the CP by manipulating PSD-95 levels conditionally only in the visual cortex was examined (Huang et al., 2015). This was accomplished by injecting an adenoassociated viral vector (AAV)-mediated gene transfer which expresses a short hairpin RNA (sRNA) targeting PSD-95 (sh95), which reduces PSD-95 expression by 90\%, which was shown in neuronal cultures.

In neurons expressing the AAV-sh95 virus, the AMPA/NMDA receptor ratio did not change over the time course of development. In contrast to that, in control neurons which were not transduced with the virus, there was a developmental increase of the AMPA/NMDA receptor 
ratio. Strikingly, the AMPA/NMDA receptor ratio of the transduced cells was similar to those of neurons in age-matched PSD-95 KO mice (Huang et al., 2015).

Comparing the fraction of silent synapses of transduced with control neurons revealed that the number of silent synapses is more than two times bigger in sh95 neurons during the critical period. Again, these values were similar to those of mice with a global KO of PSD-95. These data indicate that synaptic maturation is impaired when PSD-95 is reduced or lacking and that it is clearly cell autonomous and not due to a secondary effect of alterations in the overall network in the brain.

To examine if PSD-95 is also necessary for the stabilization of already matured silent synapses, a knockdown of PSD-95 in the VC of mice beyond the critical period (P 40) was performed, resulting in a higher number of silent synapses in comparison to control neurons. Taken together, it was clearly shown that PSD-95 is not only necessary for the maturation of silent synapses during the development, but also indispensable for the stabilization of already matured synapses, since a lack of PSD-95 after the CP could reinstate critical-period like silent synapse numbers (Huang et al., 2015).

These results allow the conclusion that there might be two different types of synapses in the visual cortex of mice: one sub pool which is dependent on PSD-95 function for the maturation during the development and for a proper stabilization after the synapses matured, and one sub pool that is independent of PSD-95 (Huang et al., 2015).

With respect to these findings and since PSD-95 KO animals show altered synaptic plasticity, most likely due to an increased number of AMPA silent synapses, interocular plasticity of vision after monocular deprivation was examined in this PhD thesis. The aim was to test whether a shift in OD of binocular neurons towards the open eye in the visual cortex can be induced by monocular deprivation (as a model for plasticity) in juvenile and adult mice. In vivo optical imaging of intrinsic signals was used to visualize cortical activity maps in V1 and plasticity in adult and old PSD-95 KO mice and their WT littermates with and without MD. 


\subsubsection{The postsynaptic density protein-93 (PSD-93)}

While PSD-95 is widely studied, only little is known about the scaffold postsynaptic density protein-93 (PSD-93), which is also a member of the MAGUK-family. There are very few publications available, especially in terms of synaptic and cortical plasticity. For a long time, PSD-93 was considered to be rather redundant to its much more abundant paralog PSD-95. It is postulated that PSD-93 is essential for surface NMDAR expression, NMDAR-mediated postsynaptic function and NMDAR-dependent persistent pain (Tao et al., 2003). PSD-93 KO mice are believed to have impaired NMDAR-mediated postsynaptic functions and are truncated in NMDAR-dependent persistent pain by mechanisms of surface NMDAR alteration (Tao et al., 2003).

McGee et al. (2001) showed that PSD-93 KO mice had no major structural or functional abnormalities and no impairments in both synaptic transmission and the associated motor behavior tested in the cerebellum. The authors claimed that MAGUK proteins like PSD-93 or PSD-95 are not required for the development of certain central synapses but may rather take part in synaptic signaling and plasticity.

Some years later, it was postulated that PSD-93 has a similar function as PSD-95 in terms of AMPA receptor mediated EPSCS, where it was shown that there is an increase of AMPA receptor EPSCs when PSD-93 is overexpressed, and a decrease of AMPAR EPSCs when it is knocked down by using Ribonucleic acid interference (RNAi) (Elias et al., 2006; Levy et al., 2015). Additionally, it was stated by Carlisle et al. (2008), that an ubiquitous KO of PSD-93 has no phenotype or impairments of basal synaptic transmission in the hippocampal CA1 region (Carlisle et al., 2008). However, they found that PSD-93 KO mice show deficits in LTP and exhibit normal LTD which is the opposite of the facilitated LTP induction and disruption of LTD observed in PSD-95 KO mice. Carlisle et al. (2008) suggest that the role of PSD-95 in AMPA receptor trafficking at excitatory synapses in the hippocampus of adult mice is unique and that PSD-93 and PSD-95 might have essentially opposing roles in LTP induction.

Other studies showed a decrease of NMDA receptor expression in the spinal cord and the forebrain (Liaw et al., 2008; Tao et al., 2003) as well as reduced NMDA receptor- mediated toxicity in cortical cultures when PSD-93 is lacking (Zhang et al., 2010), whereas AMPA receptors were thought to be unaffected in the tested brain regions. 
Krüger et al. (2013) could show that PSD-93 is the most mutable protein in the MAGUK family in terms of function and structure with six different $\mathrm{N}$-terminal isoforms. They characterized the synaptic role of PSD-93 and quantified the expression levels of all different isoforms in hippocampal synapses of mice. It was shown that PSD-93 is required at different developmental synaptic states to maintain the strength of excitatory synaptic transmission which was conducted by using a diverse number of techniques including molecular manipulations, protein overexpression, gene knockdown and knockout in combination with biochemical assays and slice electrophysiology. It is believed that the six different PSD-93 isoforms serve to fine-tune signaling scaffolds in order to regulate synaptic transmission (Krüger et al., 2013).

Since it was found that silent synapses are highly abundant in the mouse visual cortex when the scaffold protein PSD-95 is lacking (Huang et al., 2015), the laboratory under the direction of Prof. Dr. Dr. Schlüter (Dr. Favaro, unpublished data) further analyzed the relation between PSD-93 and silent synapses in mouse visual cortex. If the hypothesis that PSD-93 and PSD-95 exhibit opposing functions regarding synapse maturation is true, we would expect highly reduced silent synapse numbers in PSD-93 KO mice.

With the help of electrophysiology, Dr. Favaro (ENI, Göttingen) determined the fraction of silent synapses in brain slices of the visual cortex from PSD-93 KO and WT mice at different developmental stages. It was found that already during the critical period between P $21-30$, AMPA receptor silent synapses were mostly absent ( $1 \%)$, whereas age-matched WT control animals still exhibited $\sim 24 \%$ of silent synapses (unpublished data). In contrast to PSD-93 KO mice, silent synapses are highly abundant in age-matched PSD-95 KO mice ( $50 \%$ ), resulting in lifelong juvenile-like OD-plasticity (Huang et al., 2015). In WT mice, there is an experiencedependent gradual unsilencing of silent synapses which leads to the closure of the critical period for OD-plasticity (Huang et al., 2015).

Since silent synapses are already largely absent during the critical period in PSD-93 KO mice, we would expect opposing roles of PSD-93 and PSD-95 regarding the role of synapse maturation in the visual cortex.

If the electrophysiological findings of reduced silent synapse numbers in PSD-93 KO mice are transferrable to OD-plasticity, we would expect reduced or no OD-plasticity and a rather drastically restricted critical period. 
To investigate this, Dr. Götze performed optical imaging of intrinsic signals in PSD-93 KO mice after 4 days of MD between P 28 - 35 and after 7 days of MD between P $50-80$ in the laboratory under the direction of Prof. Dr. Löwel. In both age groups, ocular dominance indices varied strongly (ocular dominance indices were between $0.01-0.31$ ). These results did not allow any conclusion about the function of PSD-93 in OD-plasticity of mice. Therefore, Hosang (Master thesis, 2014) started to investigate OD-plasticity in PSD-93 KO mice during the critical period (P 27 - 35) and performed optical imaging after 4 days of MD. This study was continued in this present $\mathrm{PhD}$ thesis.

\subsection{Scope of the study}

PSD-95 has been largely investigated in the past years whereas PSD-93 was only subject to very few studies about synaptic plasticity. Hence, there are still many open questions about the role of the MAGUK scaffold proteins PSD-95 and PSD-93 in cortical plasticity and vision. In the postsynaptic density, the trafficking of glutamate receptors is controlled by scaffold proteins (for review see: Elias \& Nicoll, 2007) which allows alterations of synaptic strength and hence synaptic plasticity. Both, PSD-95 and PSD-93 are scaffolding proteins at the postsynaptic density which expression levels are closely correlated with the strength of AMPA receptor neurotransmission (Elias et al, 2006; Nakagawa et al 2004; Schlüter et al, 2006; Stein et al, 2003, Béïque and Andrade, 2003, Béïque et al, 2006; Huang et al., 2015).

PSD-93 was suggested to be redundant to its much more numerous paralog PSD-95 and thought to play a similar role at least in the hippocampus (Elias et al, 2006). However, other studies could not show an effect of PSD-93 in AMPA receptor neurotransmission (Carlisle et al., 2008) or in contrast a bigger effect (Levy et al., 2015). Moreover, it was not known whether PSD-93 also influences the number of silent synapses and OD-plasticity in the visual cortex as it was shown for PSD-95 (Huang et al., 2015).

Thus, with the present study, further analyses of the role of PSD-93 in synaptic plasticity and vision was conducted in cooperation with the research group under the direction of Prof. Dr. Dr. Schlüter.

Since both proteins play an important role in anchoring AMPA- and NMDA receptors (Chen et al., 2015), OD-plasticity and interocular plasticity of PSD-95 and PSD-93 KO mice and WT 
controls were tested. Furthermore, it was investigated if the loss of either PSD-95 or PSD-93 affects the CP for OD-plasticity.

Since a global knockout of PSD-95 can preserve a juvenile form of OD-plasticity lifelong (Huang et al., 2015), it was further investigated whether a knockdown (KD) of PSD-95 only in the visual cortex is already sufficient to prevent the closure of the CP for OD-plasticity and whether we can restore juvenile-like OD-plasticity also in adult WT mice.

In the hippocampus of PSD-95 KO mice, it was shown that long-term synaptic potentiation (LTP) is enhanced (Migaud et al., 1998) and that spines in PSD-95 deficient neurons are less stable after LTP induction (Ehrlich et al., 2007). Since we showed increased plasticity in PSD$95 \mathrm{KO}$ mice (Huang et al., 2015), we tested whether cortical plasticity is also less stable in PSD$95 \mathrm{KO}$ mice. Therefore, 7 days of MD were performed and the initially deprived eye reopened. After either 2- or 4 days after reopening the deprived eye, optical imaging of intrinsic signals was performed (Experiments were done together with Dr. Götze).

Recently, it was shown that by inactivating one hemisphere in mouse visual cortex, cortical activity evoked after stimulating the ipsilateral eye was significantly reduced in the binocular part of V1 of the opposing hemisphere (Dehmel \& Löwel, 2014). These data suggest that cortico-cortical interactions play a major role for determining ocular dominance in adult mouse visual cortex (Dehmel \& Löwel, 2014).

To study whether both hemispheres contributed to the OD-shift after PSD-95 knockdown in the visual cortex, PSD-95 expression was silenced either contralateral or ipsilateral to the deprived eye in WT mice and subsequently performed optical imaging of intrinsic signals. Afterwards, it was investigated whether it is feasible to reverse the maturation of excitatory synapses in V1 and therefore restore juvenile-like OD-plasticity by silencing PSD-95 in WT mice shortly after the critical period for OD-plasticity.

Additionally, it was investigated whether a knockdown of PSD-95 in the visual cortex of mice would show the same phenotype as in mice with a global PSD-95 KO (which exhibit normal visual acuity (Huang et al., 2015)) but impaired orientation discrimination (tested by Dr. Götze). Therefore, visual acuity and orientation discrimination were tested in the visual water task (VWT) (Prusky et al., 2000), which is a tasked based on reinforcement learning.

To get better insights if a loss of PSD-95 only in the visual cortex might influence visual capabilities, we also tested WT mice before and after a knockdown of PSD-95 in the VWT. 
The aim of this study was to investigate in detail the roles of PSD-95 and PSD-93 in vision and

OD-plasticity of mice with the goal to answer the following questions:

1. Is PSD-95 only required for the maturation of the brain e.g. silent synapses during the critical period or also for maintaining the matured state?

2. Do visual cortex specific PSD-95 knockdown mice show the same phenotype as ubiquitous PSD-95 KO mice?

3. Are interhemispheric interactions important for sensory processing and OD-plasticity in PSD-95 knockdown mice?

4. Can ocular dominance plasticity be restored anytime by knocking down PSD-95?

5. Can a knockdown of PSD-95 selectively only in the visual cortex also prevent the closure of the critical period for OD-plasticity?

6. Is orientation discrimination compromised after a knockdown of PSD-95 in the visual cortex of WT mice?

7. Does PSD-93 play a crucial role in vision or the critical period for OD-plasticity? 


\section{Materials and Methods}

\subsection{Animals}

\subsubsection{PSD-95 KO mice}

Male and female wild-type (WT), heterozygous (HZ) and postsynaptic density protein (PSD) 95 knockout (PSD-95 KO) mice (Yao et al., 2004) with a mixed genetic background of 129SV/C57BL/6J and WT animals with an adeno-associated-virus (AAV8) - induced PSD-95 knockdown (sh95 KD; Huang et al., 2015), as well as the respective animals serving as control groups (AAV8 - sh luciferase - GFP (shlc)) were raised from heterozygous or homozygous breeding pairs in the laboratory under the direction of Prof. Dr. Dr. Schlüter at the European Neuroscience Institute (ENI) in Göttingen, Germany. Genotyping was carried out by Dr. Huang according to the protocol provided by The Jackson Laboratory, where the DNA sample was extracted from cut tail tips (1-2 mm) digested in $150 \mu \mathrm{l}$ PBND buffer at $55^{\circ} \mathrm{C}$ overnight. Genotypes were determined by PCR with the primer pair CAGGTGCTGCTGGAAGAAGG and CTACCCTGTGATCCAGAGCTG (Huang et al., 2015; doctoral thesis: Huang, 2013).

WT mice were injected with a short hairpin ribonucleic acid (shRNA) - containing adenoassociated virus (AAV8) by Dr. Huang at postnatal days (P) $0-P 2$ or P 40 in the visual cortex of either both or only one hemisphere, respectively. Animals transduced with the same virus at $\sim P 143$ were injected together with Dr. Tippmann. The shRNA used to knock down PSD-95 was prepared by Dr. Huang as described before (Schlüter et al., 2006). The adeno-associated virus was produced as described before (Suska et al., 2013) with the shRNA construct under the control of a CAG promoter. The C57BL/6J strain was chosen because of its extensive use in laboratory studies and because it is a popular background strain for evaluating behavioral and physical changes (Prusky et al., 2004; Lehmann and Löwel, 2008). 


\subsubsection{PSD-93 KO mice}

Postsynaptic density protein (PSD) -93 knockout (PSD-93 KO; McGee et al., 2001) and WT mice as well as WT mice with an adeno associated-virus (AAV8) - induced PSD-93 knockdown (AAV - sh93), and respective animals serving as control groups (AAV - shlc) were bred in the animal facility of the European Neuroscience Institute (ENI) in Göttingen on behalf of Prof. Dr. Dr. Schlüter. All the above-mentioned genotypes exhibited a C57BL/6J background and carried two loxP sites in the synaptic associated protein (SAP) -97 gene. As they have never been crossed to a Cre driver line, the gene was considered functional and the controls as WT. PSD$93 \mathrm{KO}$ and WT mice were the progeny of hetero- or homozygous PSD-93 KO breeding pairs. Genotyping of the animals was carried out by Dr. Huang and Dr. Favaro according to the protocol provided by The Jackson Laboratory and as described in Huang et al. (2015) and the respective doctoral theses (doctoral thesis: Huang, 2013; Favaro, 2014). For knocking down PSD-93 in the visual cortex, WT mice were injected with a shRNA -containing adeno associated virus (AAV8) by Dr. Favaro at P 0 - P 2 in the visual cortex of both hemispheres.

In all experimental conditions, pups were weaned after P 21 and siblings were usually housed together in groups of maximally five mice (separated by sex) in standard cages $(33 \times 17 \mathrm{~cm})$ with 12 hours light/dark cycle with food and water provided ad libitum. All experiments were done at the Georg-August-Universität Göttingen, Germany and performed according to the German Law on the Protection of animals and the corresponding European Communities Council Directive of November 24, 1986 (86/609/EEC).

\subsection{Visual deprivation - dark rearing of PSD-93 KO and WT mice}

For visual deprivation (dark rearing (DR)), animals were born in standard cages placed into a light tight Scantainer (Scanbur Technology, Denmark) (Figure 4) in a completely light-tight darkroom until the experiments were performed between P $24-$ P 31. Mice, like other placental mammals (excluding primates), exhibit only two types of cones (possessing only $\mathrm{S}$ and $\mathrm{M}$ cone pigments) in their retina with pigments having an absorption maximum at $>500$ $\mathrm{nm}$ and S-cones with an absorption maximum at $<500 \mathrm{~nm}$. They are so called dicromats and cannot see red color (Jacobs, 2002). Therefore, animal care was done using a flashlight with a red filter (Mag-Lite, USA) generating only red and dim light to prevent light exposure of the mice. 


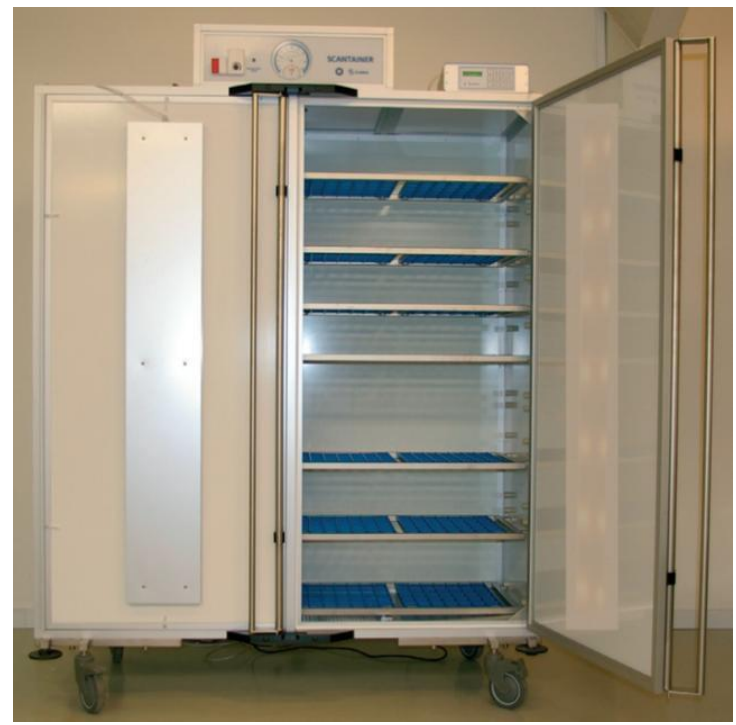

Figure 4: Dark exposure in a Scantainer (Scanbur Technology, Denmark) with light adjustment kit. The animals were raised in the Scantainer from birth in complete darkness. The lights in the animal room and in the Scantainer were always turned off. (Figure modified from www.scanburtechnology.com/fileadmin/S CBTechnology/Brochure/Scantainer_light_adjustment_kit_03.2011.pdf).

\subsection{Lifelong juvenile-like ocular dominance plasticity in PSD-95 KO mice}

One of the questions was if PSD-95 has an effect on ocular dominance plasticity in mice after either four or seven days of monocular deprivation (MD). Together with Dr. Götze, I examined WT and PSD-95 KO mice which were divided in two age groups (either < P 110 or > P 110) and tested ocular dominance plasticity after four or seven days of MD with optical imaging of intrinsic signals in vivo. Furthermore, we tested whether pharmacological enhancement of inhibition with the benzodiazepine Diazepam can prevent ocular dominance plasticity in WT and PSD-95 KO mice in vivo.

\subsection{Treatment with Diazepam}

Since PSD-95 KO mice showed a prolonged period of visual cortical plasticity (Huang et al., 2015), we assumed that this phenomenon might occur because of reduced intracortical inhibition. Therefore, we applied the drug diazepam which is a benzodiazepine enhancing the effect of the neurotransmitter $\gamma$-aminobutyric acid (GABA) by binding to the benzodiazepine site on the GABA - receptor (via the constituent chlorine atom) which leads to an increase in GABAA - mediated inhibition. Diazepam was diluted with $0.9 \%$ saline and injected intraperitoneally (i. p.; 1 gg / g mouse) daily for seven days during the MD - period. In this 
study, we used a dosage of $1 \mu \mathrm{g}$ drug per gram mouse. In our hands, a dose of $30 \mu \mathrm{g}$ drugs per mouse gram that was used by other groups before, was lethal (Hensch et al., 1998; Huopaniemi et al., 2004; Kanold et al., 2009).

\subsection{Neural circuits are less stable in PSD-95 KO mice}

To test whether cortical plasticity is more transient in PSD-95 KO mice in comparison to WT, optical imaging of intrinsic signals was performed after seven days of MD in both genotypes, the initially deprived eye was re-opened and visual cortical activity was imaged two or four days after reopening the former closed eye.

Bianka Götze tested 33 animals and I tested 28 animals equally distributed in the WT and PSD$95 \mathrm{KO}$ groups without MD and after either 4 or 7 days of MD. For the reopen experiments, Bianka Götze tested 20 animals and I tested 22 animals also equally distributed across the different groups. 
A detailed description of the experimental groups is displayed in Table 1.

Table 1: Description of the experimental groups used in this study to test ocular dominance plasticity in WT and PSD-95 KO mice. The name of the respective group as well as the age range and the total number of animals per group are listed.

\begin{tabular}{|c|c|c|c|c|}
\hline genotype & condition & $\begin{array}{c}\text { age range } \\
\text { (days) }\end{array}$ & $\begin{array}{l}\text { average age } \\
\text { (days) }\end{array}$ & $\begin{array}{c}\text { number of } \\
\text { mice per } \\
\text { group }\end{array}$ \\
\hline WT & no $M D$ & $97-488$ & 286 & 6 \\
\hline PSD-95 KO & no $M D$ & $83-496$ & 229 & 7 \\
\hline WT & $7 \mathrm{~d} M D$ & $74-82$ & 78 & 9 \\
\hline WT & $7 \mathrm{~d} M D$ & $123-311$ & 215 & 7 \\
\hline PSD-95 KO & $7 \mathrm{~d} M D$ & $70-99$ & 85 & 8 \\
\hline PSD-95 KO & $7 \mathrm{~d} M D$ & $232-507$ & 333 & 7 \\
\hline PSD-95 KO & $4 \mathrm{~d} M D$ & $268-480$ & 337 & 7 \\
\hline WT & $\begin{array}{c}7 \mathrm{~d} \text { MD } \\
+ \text { Diazepam }\end{array}$ & $80-106$ & 100 & 5 \\
\hline PSD-95 KO & $\begin{array}{c}7 \mathrm{~d} \text { MD } \\
+ \text { Diazepam }\end{array}$ & $83-123$ & 101 & 4 \\
\hline WT & $2 \mathrm{~d}$ reopen & $77-84$ & 81 & 4 \\
\hline WT & $4 \mathrm{~d}$ reopen & $88-97$ & 94 & 3 \\
\hline PSD-95 KO & $2 \mathrm{~d}$ reopen & $76-83$ & 80 & 5 \\
\hline & & & total & 72 \\
\hline
\end{tabular}




\subsection{V1-specific reduction of PSD-95 expression restores juvenile-like ocular dominance plasticity}

After testing OD-plasticity in ubiquitous PSD-95 KO mice, it was investigated whether a knockdown of PSD-95 only in the visual cortex would be sufficient to prevent the closure of the critical period for OD-plasticity. Additionally, it was tested whether the PSD-95 dependent rejuvenation of the excitatory synapses in V1 can restore critical-period like OD-plasticity in adult WT mice.

To this end, PSD-95 was selectively knocked down in the visual cortex of either P 0 - P 2 pups or adult (P 40, P 143) mice with an adeno-associated virus (AAV - sh95) targeting PSD-95 or controls (AAV - shlc). All mice were tested for ocular dominance plasticity with optical imaging of intrinsic signals after four days of MD.

Animals injected with sh95 or shlc at P 143, were tested in the visual water task (VWT) for visual acuity and orientation discrimination before and after the virus injections and then imaged chronically after four and seven days of monocular deprivation, respectively.

A detailed description of the experimental groups is displayed in Table 2. 
Table 2: Description of the experimental groups used in this study to test ocular dominance plasticity in WT mice with a knockdown of PSD-95 in the visual cortex at P 0, P 40 or P 143. The name of the respective group as well as the age range and the total number of animals per group are listed.

\begin{tabular}{|c|c|c|c|c|}
\hline virus injection & condition & $\begin{array}{c}\text { age } \\
\text { range } \\
\text { (days) }\end{array}$ & $\begin{array}{c}\text { average } \\
\text { age (days) }\end{array}$ & $\begin{array}{l}\text { number } \\
\text { of mice } \\
\text { per } \\
\text { group }\end{array}$ \\
\hline P 0 - P 2, shlc in both $h$. & $4 \mathrm{~d} M D$ & $104-105$ & 104 & 4 \\
\hline $\mathrm{P} 0-\mathrm{P} 2$, sh95 in both $\mathrm{h}$. & $4 \mathrm{~d} M D$ & $29-181$ & 74 & 7 \\
\hline P 0-P 2, sh95 in left $h$. & $4 \mathrm{~d} M D$ & $70-104$ & 91 & 7 \\
\hline$P 0-P 2$, sh95 in right $h$. & $4 \mathrm{~d} M D$ & 73-105 & 95 & 5 \\
\hline P 40, shlc in both $h$. & $4 \mathrm{~d} M \mathrm{MD}$ & $72-82$ & 77 & 8 \\
\hline P 40, sh95 in both $h$. & $4 \mathrm{~d} M D$ & $76-116$ & 83 & 10 \\
\hline \multirow[t]{2}{*}{ P 143, shlc in both h. } & $4 \mathrm{~d} M D$ & $198-215$ & 205 & 5 \\
\hline & $7 \mathrm{~d} M D$ & $201-218$ & 200 & 3 \\
\hline \multirow[t]{2}{*}{ P 143 , sh95 in both h. } & $4 \mathrm{~d} M \mathrm{MD}$ & $182-208$ & 200 & 6 \\
\hline & $7 \mathrm{~d} M D$ & $185-211$ & 203 & 6 \\
\hline & & & total & 52 \\
\hline
\end{tabular}

\subsection{The role of PSD-93 on ocular dominance plasticity and vision in mice}

As nothing about visual functions and the visual cortex of PSD-93 KO mice was published so far, it was unknown whether PSD-93 plays a crucial role in vision or the CP for OD-plasticity in mice, like PSD-95 does.

Therefore, the effect of PSD-93 on ocular dominance plasticity in mice during the critical period after four days of MD and on vision in adult (P 60) mice was investigated. Together with Leon Hosang, I examined WT and PSD-93 KO mice which were divided into two age groups (mid critical period: P 24 - P 27; late critical period: P 28-P 35). All animals got tested in the optomotor setup (Prusky et al., 2004) to examine their baseline threshold of the optomotor reflex and contrast sensitivity before and during the four days of the MD - period. 
After four days of MD, optical imaging was performed to test for ocular dominance plasticity. A detailed description of the experimental groups is displayed in Table 3:

Table 3: Description of the experimental groups used in this study to test ocular dominance plasticity in WT and PSD-93 KO mice during the critical period. The name of the respective group as well as the age range and the total number of animals per group are listed.

\begin{tabular}{|c|c|c|c|c|}
\hline genotype & condition & $\begin{array}{c}\text { age range } \\
\text { (days) }\end{array}$ & $\begin{array}{r}\text { average age } \\
\text { (days) }\end{array}$ & $\begin{array}{r}\text { number of } \\
\text { mice per } \\
\text { group }\end{array}$ \\
\hline \multicolumn{5}{|c|}{ mid critical period: P24-27 } \\
\hline WT & no MD & $25-27$ & 26 & 3 \\
\hline WT & 4 d MD & $24-26$ & 25 & 4 \\
\hline PSD-93 KO & no MD & $24-27$ & 26 & 6 \\
\hline PSD-93 KO & 4 d MD & $24-27$ & 26 & 5 \\
\hline \multicolumn{7}{|c|}{} & no MD & $29-34$ & 32 & 7 \\
\hline WT & 4 d MD & $29-35$ & 32 & 5 \\
\hline WT & no MD & $28-31$ & 30 & 41 \\
\hline PSD-93 KO & 4 d MD & $30-35$ & 32 & total \\
\hline PSD-93 KO & & &
\end{tabular}

Leon Hosang tested 12 animals during the mid critical period and 11 animals during the late critical period. I tested 6 animals during the mid critical period and 12 animals during the late critical period.

Additionally, I tested PSD-93 KO $(n=11)$ and WT $(n=8)$ in the visual water task for visual acuity and orientation discrimination $(n=5 / n=4)$.

\subsection{V1-specific reduction of PSD-93 expression in V1 of WT mice from birth}

After testing OD-plasticity in ubiquitous PSD-93 KO mice, I investigated whether a knockdown of PSD-93 only in the visual cortex would also result in an earlier closure of the critical period for OD-plasticity in WT mice. To this end, PSD-93 was selectively knocked down in the visual cortex of WT (P 0 - P 2) pups with an adeno-associated virus (AAV - sh93) targeting PSD-93 or 
controls (AAV - shlc). All animals were tested for ocular dominance plasticity with optical imaging of intrinsic signals after four days of $\mathrm{MD}$ or without $\mathrm{MD}$ in controls during the late $\mathrm{CP}$ (P 29 - 35). A detailed description of the experimental groups is displayed in Table 4.

Table 4: Description of the experimental groups used in this study to test ocular dominance plasticity in WT mice with a knockdown of PSD-93 in the visual cortex at P 0-P 2. The name of the respective group as well as the age range and the total number of animals per group are listed.

\begin{tabular}{|c|c|c|c|c|}
\hline genotype & $\begin{array}{c}\text { Condition } \\
\text { virus } \\
\text { injection P0- } \\
\text { P2 }\end{array}$ & $\begin{array}{c}\text { age range } \\
\text { (days) }\end{array}$ & $\begin{array}{c}\text { average age } \\
\text { (days) }\end{array}$ & $\begin{array}{c}\text { number of } \\
\text { mice per } \\
\text { group }\end{array}$ \\
\hline WT & no MD + shlc & $34-35$ & 35 & 3 \\
\hline WT & 4 d MD + shlc & $29-30$ & 29 & 3 \\
\hline WT & no MD + sh93 & $31-35$ & 33 & 3 \\
\hline WT & 4 d MD + sh93 & $30-34$ & 32 & 6 \\
\hline
\end{tabular}

\subsection{Monocular deprivation}

To test visual cortical plasticity of mice, monocular deprivation (MD) was performed by suturing the right eyes of mice according to already published protocols (Gordon and Stryker, 1996; Cang et al., 2005; Lehmann and Löwel, 2008). To this end, mice were anesthetized in an acrylic plastic box with $2 \%$ of the anesthetic gas isoflurane in a $1: 1$ mixture of nitrous oxide (N2O) and oxygen (O2). After full general anesthesia of the mouse was reached (which was verified by performing a toe-pinch test), the mouse was placed on a heating pad to maintain body temperature at $37^{\circ} \mathrm{C}$. The isoflurane gas concentration was reduced to $1 \%$ in $1: 1 \mathrm{O}_{2}: \mathrm{N}_{2} \mathrm{O}$ and anesthesia was applied through a tube attached to the nose of the mouse. The eyelids were trimmed and the eye carefully flushed with $0.9 \%$ saline (BBraun, Melsungen). Afterwards, an antibiotic gel (Gentamycin gel, active agent: $0.3 \%$ gentamycin sulfate; medphano, Berlin) was applied on top of the right eye to avoid inflammation. The lid margins were then closed by using 7-0 silk (Ethicon, Norderstedt) with two mattress sutures (Figure 5). 


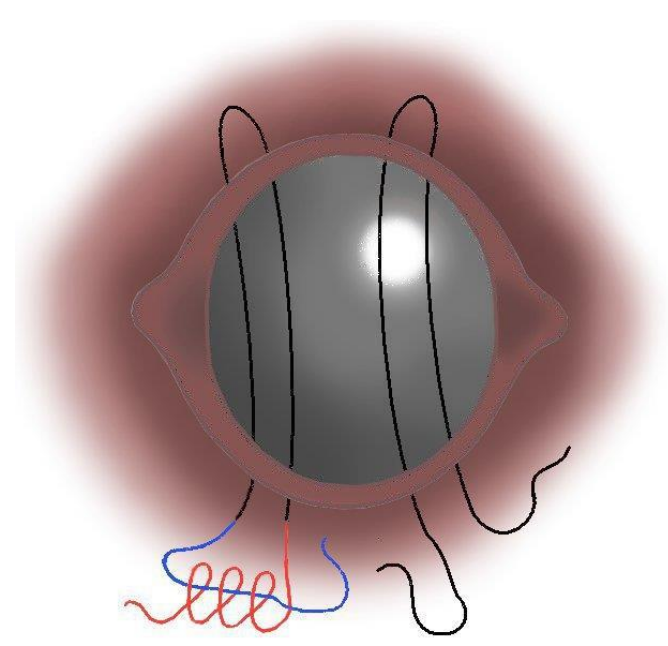

Figure 5: Procedure of monocular deprivation in mice. The lid margins of the mice were closed by using 70 silk (Ethicon, Norderstedt) with two mattress sutures.

After the procedure, the analgesic Rimadyl (5 $\mathrm{g}$ / g mouse; active agent: Carprofen; Pfizer, Berlin) diluted in $0.2 \mathrm{ml} 0.9 \%$ saline was injected intraperitoneally (i.p.) and the mice were brought back to their standard home cages and placed under a red heating lamp until they fully recovered from anesthesia. All mice were checked daily to guarantee that the deprived eye remained closed and uninfected over the MD - period of either 4 or 7 days. In the rare case of an open eye which was previously sutured, the animal got excluded from further experiments.

\subsection{Quantification of visual capabilities in a behavioral task}

\subsubsection{The virtual-reality optomotor system (Prusky et al., 2004)}

Spatial vision of mice can be examined by using the virtual-reality optomotor system (Prusky et al., 2004) (Figure 6 A, B). This test does not demand any specific training of the mice since it is based on the optomotor reflex in response to a moving, sinusoidal stimulus. To quantify spatial vision, both the spatial frequency threshold of the elicited optomotor reflex (referred to as "visual acuity") and the contrast threshold (referred to as "contrast sensitivity") were tested. To test this, a mouse is placed on a platform (13 cm high, $5.3 \mathrm{~cm}$ in diameter) surrounded by four monitor screens ( $33.5 \mathrm{~cm}$ width $\times 6.5 \mathrm{~cm}$ height) with orthogonal screens facing each other, forming a square box virtual cylinder showing $360^{\circ}$ moving vertical sine wave gratings of different spatial frequencies and contrasts generated by 
the software OptoMotry 1.4.0 (CerebralMechanics, Lethbridge, Alberta, Canada) which was previously reported (Prusky et al., 2004; Lehmann and Löwel, 2008).

The mouse can move completely voluntarily and freely on the platform and is relocated on the platform in case it jumps down.

On the floor and on the ceiling of the apparatus, there are mirrors installed, while a video camera (FireWire iSight; Apple Computer Corp., Mountain View, Canada) placed in the lid of the apparatus records the animals' behavior from above. The camera is connected to an external monitor screen such that the experimenter can observe the behavior of the mouse without disturbing it (Figure 6; A, B).

A

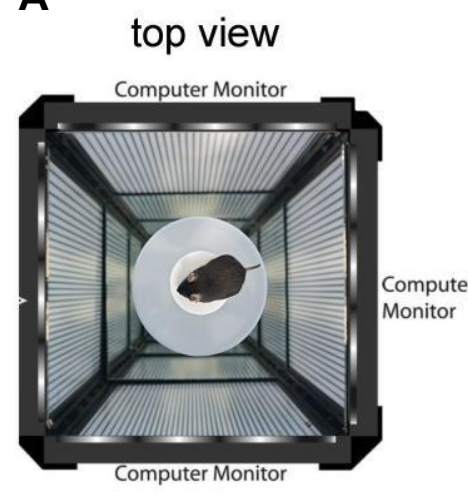

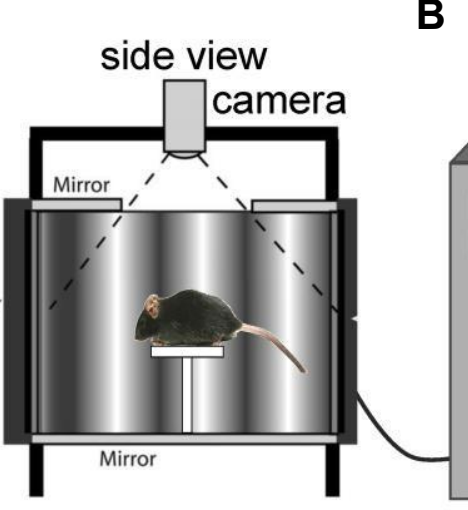

B

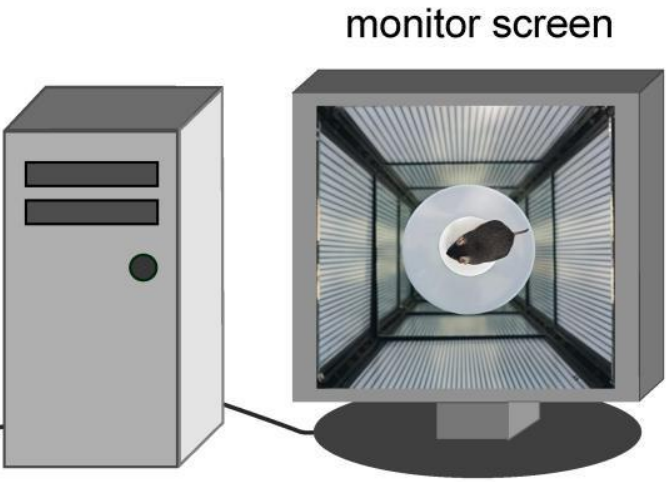

Figure 6: Schematic of the behavioral testing optomotor apparatus. (A) Top view. The mouse is surrounded by $360^{\circ}$ of moving sinusoidal gratings. (B) Side view. The mouse can freely move on a platform which is positioned in the middle of the testing apparatus consisting of four monitor screens. Sinusoidal sine wave gratings on the screens are extended vertically with mirrors on the ceiling and floor to create a virtual cylinder. The animal's behavior is recorded with a video camera from above connected with an external monitor screen. (Figure modified from Prusky et al., 2004).

To achieve a higher accuracy of the measurements, the center of the rotating virtual cylinder was fixated manually with the $x-y$ coordinates of crosslines between the eyes of the animal using the camera recordings, so that the set parameters and the distance from the grating to the eyes of the mouse was kept constant (Figure 7). 
A

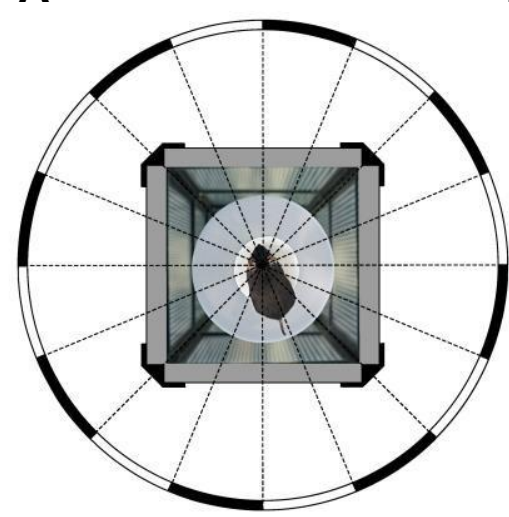

B

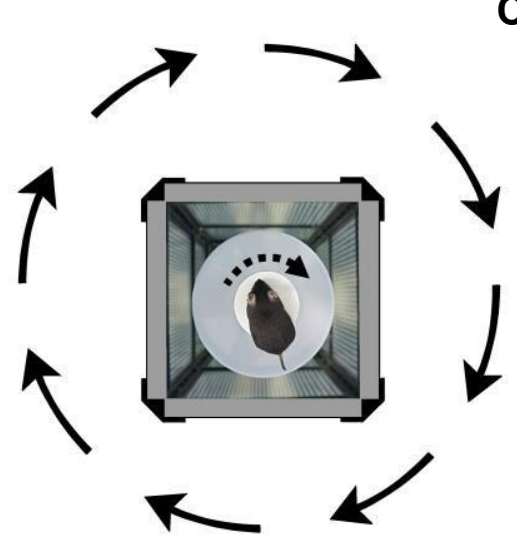

C

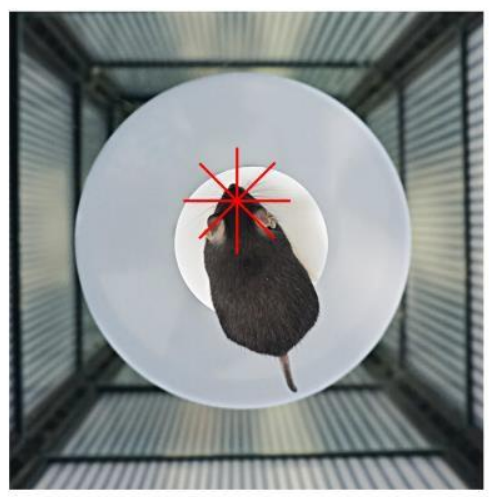

Figure 7: Virtual cylinder and optomotor response (Figures modified from Prusky et al., 2004). (A) In a 3dimensional coordinate space, a virtual cylinder is projected on all four monitor screens. The center of the rotating cylinder is determined by the location of the head of the mouse. (B) When the cylinder is rotating either clock- or anti-clockwise, the mouse tracks the drifting sine wave grating with reflexive neck and head movements. (C) A video camera image of a mouse tracking the moving sine wave grating. The red crosslines are positioned between the eyes of the mouse such that the coordinates can be used to center the rotation of the virtual cylinder.

Mice will reflexively track the moving vertical sine wave gratings by neck and head movements if they can see the gratings (Figure 7 B). The tracking behavior can only be elicited in the temporal-to-nasal direction which makes it possible to measure the spatial frequency threshold of the optomotor reflex for both eyes separately by reversing the direction of the moving sine wave grating (Douglas et al., 2005). The spatial frequency or contrast threshold of the left eye can be measured with clockwise moving gratings, whereas the threshold of the right eye can be measured by gratings moving counterclockwise.

In all mice, the spatial frequency threshold at $100 \%$ contrast and contrast at six different spatial frequencies $(0.031$ cycles / degree (cyc / deg), 0.092, 0.103, 0.192, 0.272) with a drifting speed of $12^{\circ}$ per second $\left(12^{\circ} / \mathrm{s}\right)$ were measured. For the spatial frequency threshold of the optomotor reflex, the spatial frequency was increased until the tracking of the mouse was not detectable anymore. For contrast sensitivity, the contrast for each of the six spatial frequencies was lowered until the mouse did not show any tracking behavior anymore. The optokinetic response threshold for contrast was calculated for each spatial frequency separately as a Michelson contrast from the screen luminance where the factor 0.997 lux refers to the maximum contrast of the screen according to following equation: 


$$
\begin{gathered}
0.997 \text { lux }=\frac{\text { (black luminescence }- \text { white luminescence })}{\text { (black luminescence }+ \text { white luminescence) }} \\
\text { contrast sensitivity }=\frac{100}{\text { contrast (measured value in \%) x 0.997 lux }}
\end{gathered}
$$

To measure the optokinetic response threshold (visual acuity and contrast sensitivity), mice were tested daily during the period of monocular deprivation to detect potential changes of the threshold of the remaining open eye throughout the MD -period of 4 - 7 days.

The optomotor system is a very fast tool to measure the optokinetic response thresholds for spatial frequencies and contrasts and does not require any training of the animals and can be performed in mice at any age. However, since it is based on subcortical pathways, we cannot detect the real visual acuity limit of the mice. For this reason, PSD-93 KO and mice with a visual cortex restricted knock-down of PSD-95 were also tested in the visual water task. 


\subsubsection{Visual water task}

As a second, very reliable method to assess visual acuity in mice, the so called visual water task was used. This is a visual discrimination task that is based on reinforcement learning (Prusky et al., 2000; Prusky et al., 2004; Prusky and Douglas, 2004) (Figure 8 A-B).

A

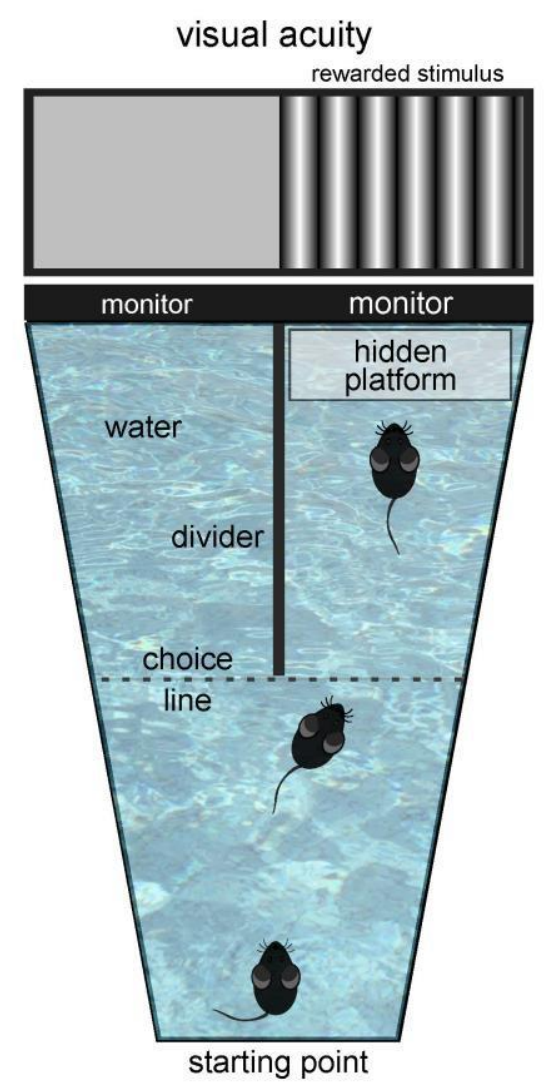

B

\section{front view}

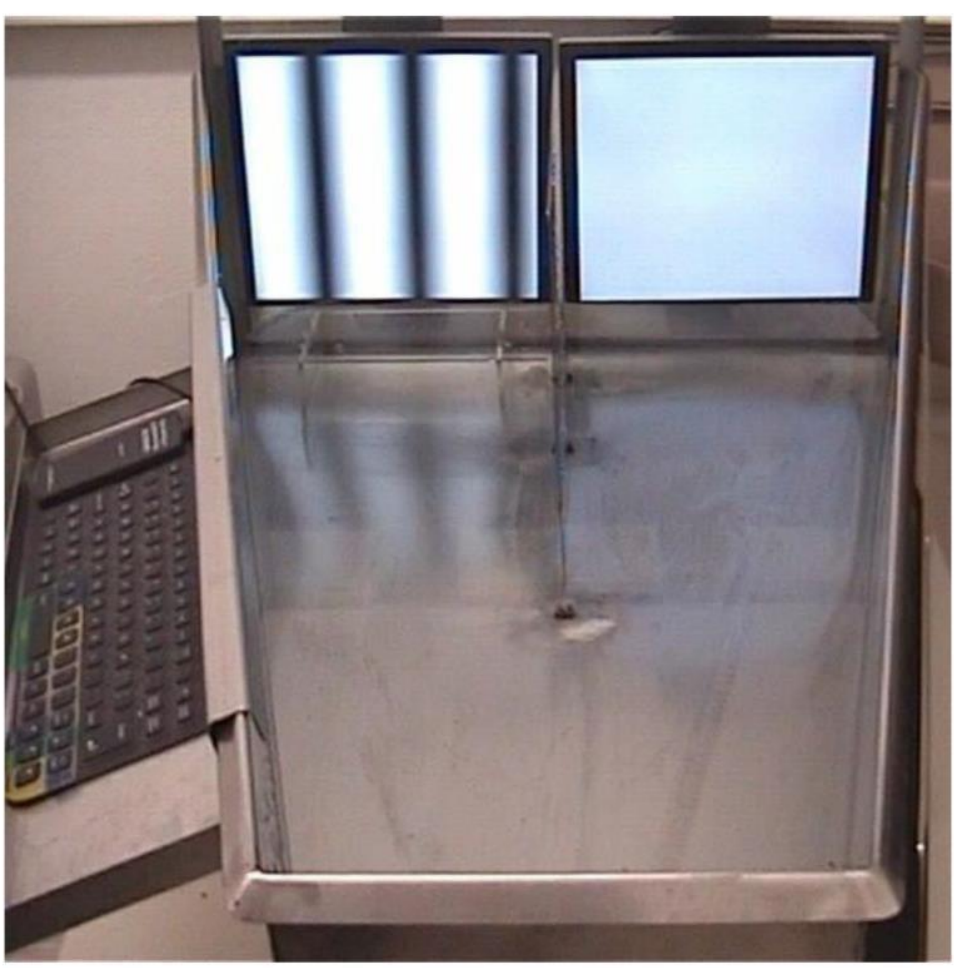

Figure 8: The visual water task. (A) Top view from the visual water task which consists of a big water tank, a midline divider, two monitor screens and a transparent platform. The pool is filled with water until the platform is fully covered and hence invisible for the mice. From the starting point at the beginning of the tank, animals learn to swim to one side of the pool on which the vertical stimulus is presented on the monitor screen. The reward is the hidden platform from where the mice are returned to their holding boxes. (B) Front view of two monitor screens, the hidden platform on the left and the midline divider. The stimulus screen is presenting a vertical, sinusoidal stationary grating whereas the reference screen projects only isoluminent grey with the same brightness intensity as the stimulus screen.

The main component of the visual water task is a trapezoidal steel tank measuring $120 \mathrm{~cm}$ in length, $40 \mathrm{~cm}$ in height and $25 \mathrm{~cm}$ width at the starting point and $80 \mathrm{~cm}$ and the end of the tank. The pool is fully divided with a steel plate at the beginning of the training and later 
partially divided with a plate of $45 \mathrm{~cm}$ length. The back of the steel tank is made of translucent acrylic plastic covering two identical 17 -inch monitor screens facing the inner side of the tank. By dividing the pool in the middle with the steel plate, two alleys are created with each ending in front on one monitor screen located in $15 \mathrm{~cm}$ height regarding the tank bottom. Both screens are connected to an Apple MacPro creating a stationary, vertical sinewave grating with a low spatial frequency of $0.086 \mathrm{cyc} / \mathrm{deg}$ on the stimulus screen and an isoluminent grey background on the reference screen, both generated by the software Vista $X$ 3.5.004 (CerebralMechanics, Lethbridge, Alberta, Canada).

The tank was filled with lukewarm water to a level of $15 \mathrm{~cm}$ to fully cover a translucent platform placed in front of the stimulus screen (33 cm length, $13 \mathrm{~cm}$ width and $14 \mathrm{~cm}$ height). Mice are instinctive swimmers (Prusky et al., 2000) and were trained to swim to the stimulus screen. The hidden platform underneath the water surface served as a reward to get out of the water. The high reflection level of the illuminating screens ensured that the mice were not able to see the platform underneath the water surface and experiments were performed in darkness to insure better visibility of the two monitor screens.

In the visual water task, mice were released into the water at the starting point (Figure 8, A) and trained to distinguish between the two monitor screens. When reaching the rewarded stimulus screen under which the hidden platform was located, the animals were immediately taken out of the water and returned to their holding cage - an acrylic plastic box bedded with tissue placed on a heating pad to prevent cooling and to accelerate fur drying. When an animal was swimming to the reference screen instead of swimming to the rewarded stimulus screen, it had to swim back around the partial divider in order to finally reach the rewarding platform. This additional time the mice had to spent in water is considered as a penalty since mice usually prevent being in water.

The position of the sinusoidal grating with the platform is alternated in a pseudorandom sequence over the training and test trials.

\subsubsection{Determination of the maximum spatial frequency threshold}

To test visual acuity, mice had to be trained to discriminate a stationary, vertical sinusoidal grating of alternating black and white bars displayed on one screen (rewarded stimulus) from an isoluminent gray which was displayed on the other monitor screen (reference screen). The 
mean of the luminescence values of the black and white bars was equal to that of the luminescence value of the gray screen.

Before the actual testing phase, mice had to be trained in the visual water task. The training phase was divided into two parts: the pre-training phase in which animals were accustomed to the general conditions of the task (Figure 9) and the training phase.

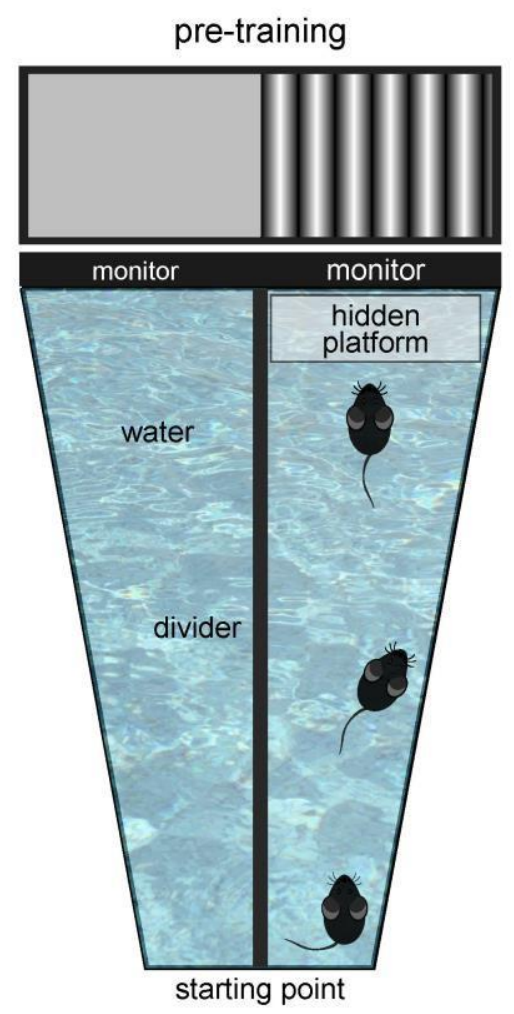

Figure 9: Pre-training phase in the visual water task. The water tank is fully divided by a metal plate and mice are released into the water at the starting point and trained to swim towards the stimulus screen showing a sinusoidal vertical grating of 0.086 cycles per degree. As soon as the animals reach the hidden platform underneath the water surface positioned below the monitor screen, they are returned to their heated holding boxes.

During the pre-training phase, the water tank was divided into two parts using the long divider spanning the whole length of the pool. The monitor screen displayed the stimulus at full contrast and lowest spatial frequency (0.086 cyc / deg) and the translucent platform was placed in front of the screen underneath the water surface. The mice were then released into the water at the starting point and returned to their warmed acrylic holding box immediately after reaching the platform. The pre-training phase took up to three days until the animals 
were fully habituated to this new environment. Each animal swam 5 times on the first day, 10 times on the second day and 15 times on the third day.

After the initial pre-training phase, animals had to learn to discriminate the stimulus screen from the isoluminent gray during the training phase. Therefore, the water tank was then divided only until the midline of the pool, creating a choice line, such that the mouse had to decide in which direction to swim. Contrast was set at maximum and spatial frequency at minimum (0.086 cyc / deg). The stimulus was displayed in a pseudorandom manner on the left $(L)$ or right $(R)$ screen following the pattern LRLRRLRLL, repeatedly. This pseudorandom pattern prevented the mice from simply learning the order of the position where the screen was displaying the rewarded stimulus. The animals were trained daily in two blocks of 15 consecutive trials each. Between the two blocks, a break of at least 90 minutes was maintained which allowed the mice to recover. During the break, mice were returned to their home cages for water and food supply. In case an animal developed a tendency to swim either always left or right, a pattern displaying the stimulus twice as often at the non-biased side was used to counteract for that. If a mouse achieved accuracy of at least $90 \%$ correct decisions in 3 consecutive blocks of 10 trials each, it was conveyed to the final testing phase. In the final testing phase, the settings and implementation were generally the same as for the training phase. However, the spatial frequency was then increased stepwise and the animals had to pass a specific criterion of swimming at least $70 \%$ correct in a block of 10 trials to reach the next higher frequency. In case an animal did not pass the $70 \%$ correct swims criterion anymore, the spatial frequency was lowered by three steps at once, which was defined as a so called 'break'. If a mouse reached three breaks at an identical or very similar spatial frequency, the experiment was completed for that specific animal. A break is defined as the spatial frequency of the grating on the stimulus screen which the mouse cannot distinguish from the isoluminent grey screen anymore. The spatial frequencies of the steps prior to each of the breaks were then used to calculate the mean visual acuity threshold, which was then defined as the spatial frequency the animal was still able to perceive. The rules underlying the test phase were as follows: 
Table 5: Criterion rules to pass to the next higher spatial frequency in the testing phase.

\begin{tabular}{|c|c|c|c|}
\hline \multirow[b]{2}{*}{ Test phase: } & \multicolumn{3}{|c|}{ Spatial frequency range in cycles per degree (cyc/deg) } \\
\hline & $0.086-0.172$ & $0.201-0.372$ & $\geq 0.401$ \\
\hline Criterion to pass & $1 / 1$ & $3 / 3$ & $5 / 5$ \\
\hline to the next higher & $3 / 4$ & $5 / 6$ & $7 / 10$ \\
\hline (cyc/deg): number & $7 / 10$ & $7 / 10$ & \\
\hline $\begin{array}{l}\text { of correct trials / } \\
\text { number of trials }\end{array}$ & $\begin{array}{l}\text { not fulfilled: } \\
\qquad \rightarrow \text { break } \\
\text { lowered spatial } \\
\text { frequency of } 3 \\
\text { steps }\end{array}$ & $\begin{array}{l}\text { not fulfilled: } \\
\qquad \rightarrow \text { break } \\
\text { lowered spatial } \\
\text { frequency of } 3 \\
\text { steps }\end{array}$ & $\begin{array}{l}\text { not fulfilled: } \\
\qquad \rightarrow \text { break } \\
\text { lowered spatial } \\
\text { frequency of } 3 \\
\text { steps }\end{array}$ \\
\hline
\end{tabular}




\subsubsection{Determination of the minimum angle difference in the visual water task}

Besides measuring visual acuity of the mice in the visual water task, one can also determine the ability of the mice to discriminate stimuli of different orientations (orientation discrimination). To this end, mice were trained to swim towards a vertical square wave grating of alternating black and white bars on the stimulus screen, while on the reference screen a horizontal square wave grating of the same spatial frequency was presented. The reference stimulus was then rotated in $5^{\circ}$ steps throughout the experiment, thereby becoming more and more similar to the vertical stimulus the mice were trained for (Figure 10).

\section{orientation discrimination}

rewarded stimulus

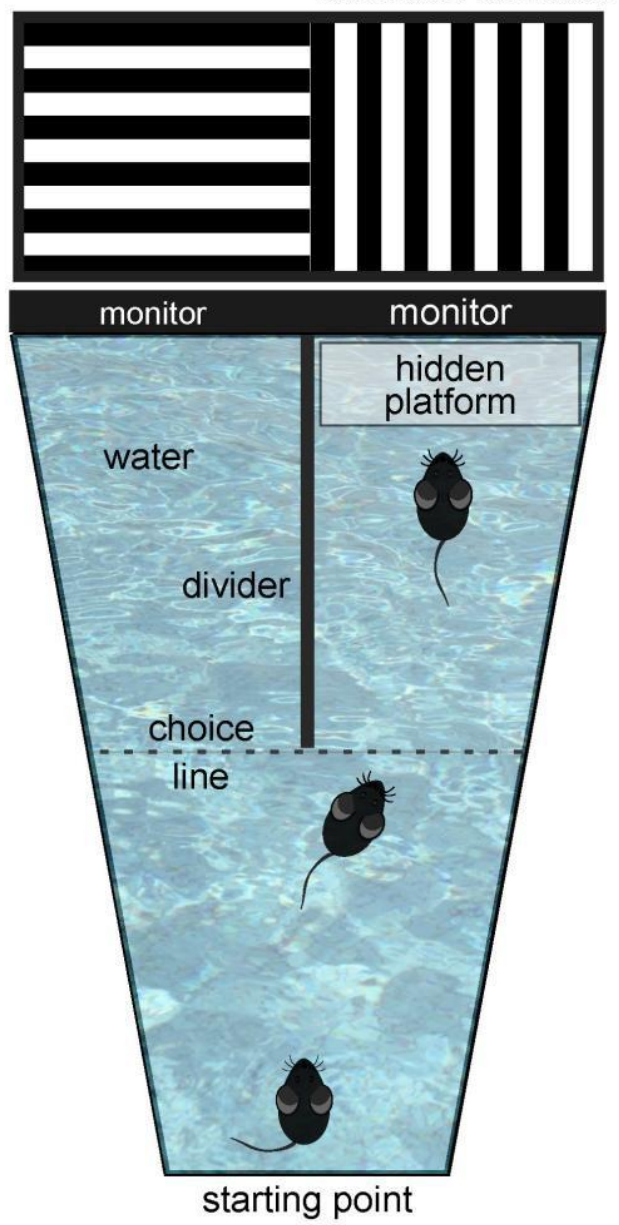

Figure 10: schematic overview of testing orientation discrimination in the visual water task. To test orientation discrimination, mice were trained to discriminate between a horizontal square wave grating (rewarded stimulus) from a vertical square wave grating (reference screen). The angle of the horizontal grating on the reference screen is adjusted to the vertical grating in $5^{\circ}$ steps until the animals were not able to distinguish the orientations of the gratings on the two screens anymore. (Figure modified from Prusky et al., 2000). 
Since the mice had already been tested for visual acuity in the VWT and were used to the general conditions of this behavioral task, no pre-training was required for testing the orientation discrimination and the experiment was only subdivided into training- and testing phases. The rules underlying the testing phase were as follows (Figure 11):

\begin{tabular}{|c|c|c|c|}
\hline \multirow{2}{*}{ Testing phase } & \multicolumn{3}{|c|}{ Angle difference of reference stimulus vs. rewarded stimulus in ${ }^{\circ}$} \\
\hline & $90-75$ & $60-45$ & $30-5$ \\
\hline \multirow{3}{*}{$\begin{array}{l}\text { Criterion to pass } \\
\text { the next higher } \\
\text { step ( } 5^{\circ} \text { rotation) }\end{array}$} & $1 / 1$ & $3 / 3$ & $5 / 5$ \\
\hline & $3 / 4$ & $5 / 6$ & $7 / 10$ \\
\hline & $7 / 10$ & $7 / 10$ & \\
\hline \multirow{4}{*}{$\begin{array}{l}\text { Number of correct } \\
\text { trials / number of } \\
\text { trials }\end{array}$} & not fulfilled: & not fulfilled: & not fulfilled: \\
\hline & $\rightarrow$ break & $\rightarrow$ break & $\rightarrow$ break \\
\hline & $15^{\circ}$ rotation & $15^{\circ}$ rotation & $15^{\circ}$ rotation \\
\hline & backwards & backwards & backwards \\
\hline
\end{tabular}

angle difference

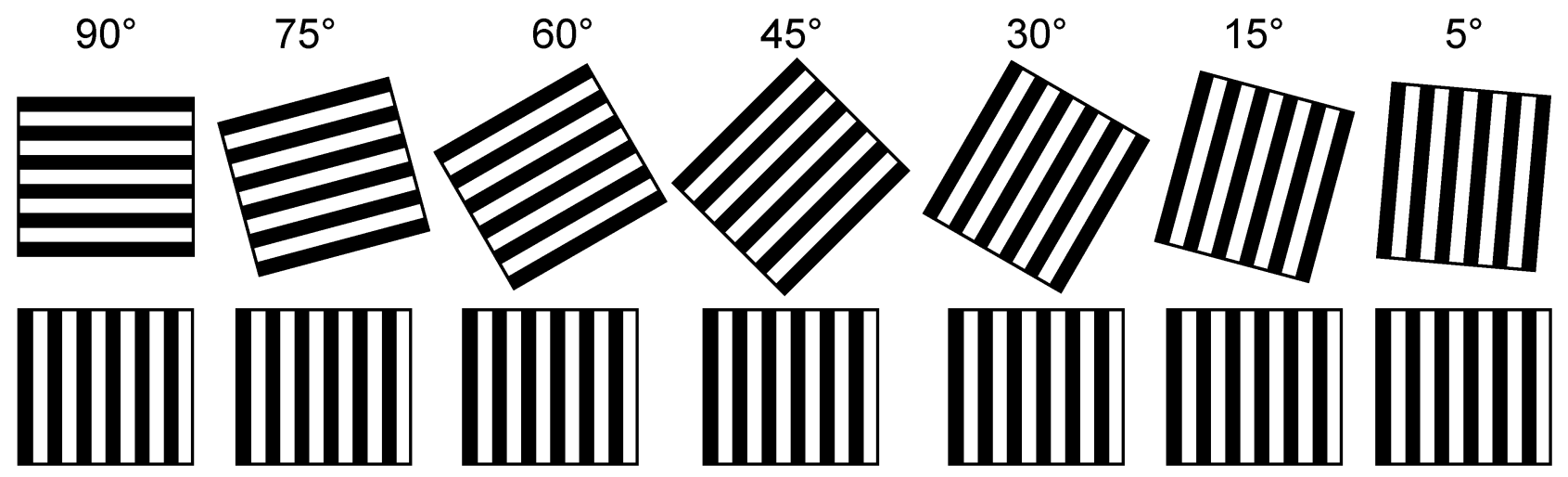

Figure 11: Angle difference between test stimulus and reference stimulus. Upper row: Reference stimulus. Lower row: rewarded test stimulus. Dependent on the animal's accuracy, the orientation of the reference stimulus was rotated in $5^{\circ}$ steps thereby increasing the resemblance of both stimuli orientations. When achieving $70 \%$ correct decisions in one block, the difference of the orientations was increased by $5^{\circ}$ steps and in case of failing the $70 \%$ correct swims criterion, it was decreased by steps of $15^{\circ}$. 


\subsection{Surgical preparations for optical imaging of intrinsic signals in vivo}

Surgery and preparation of the test animals were largely performed according to the protocol developed by Kalatsky and Stryker (2003).

At first, the animal was anesthetized with $2 \%$ of the narcotic gas Halothane in a $1: 1$ mixture of $\mathrm{O}_{2} / \mathrm{N}_{2} \mathrm{O}$ in an acrylic plastic box bedded with tissue. To achieve general anesthesia, gas inhalation with halothane is superior to most injectable drugs in both safety and efficacy, since it is easy to adjust the anesthetic depth. Furthermore, the anesthetics are eliminated from the blood by exhalation and do not rely on drug metabolism for being removed from the body. Additionally, there is less chance for drug-induced toxicity. The dosage of gas anesthesia can vary among individual animals and anesthesia was therefore administered to effect.

To test if the animal reached the general anesthesia level, a toe pinch test was performed. If no reflex could be elicited, the animal was placed on a heating pad and the halothane $-\mathrm{O}_{2} /$ $\mathrm{N}_{2} \mathrm{O}$ supply was maintained with a tube attached to the nose of the animal. The halothane concentration was then lowered to $1 \%$ and the toe-pinch test was performed consecutively over the whole course of the experiment to insure general anesthesia. For administering the correct amount of drugs before the surgery, I calculated the correct drug dosages according to the animal's weight. The antipsychotic and sedative drug chlorprothixene $(5 \mathrm{mg} / \mathrm{kg}$ body weight; Sigma-Aldrich, Steinheim, Germany) was injected into the adductor muscle of the right hind leg. The immunosuppressant glucocorticosteroid dexamethasone, which decreases inflammation and prevents brain swelling, was applied with a dosage of $5 \mathrm{mg} / \mathrm{kg}$ body weight subcutaneously (s.c.) (reactive agent dexamethasonedihydrophosphate-dinatrium, Merck Serono, Darmstadt, Germany) as well as atropine (3 mg / kg body weight; s.c.; Köhler-Chemie, Bensheim, Germany), a drug used as cycloplegic to dilate the pupils, to prevent low heart rates during intubation and to inhibit saliva production and mucus by acting on the parasympathetic nervous system. Finally, $0.9 \%$ sodium chloride solution $(0.2 \mathrm{ml}$; B Braun, Melsungen, Germany) was injected (s.c.) to prevent dehydration of the animal and maintaining the animal's water-and electrolyte- balance throughout the experiment.

After injecting the drugs, the mouse was placed in a stereotaxic frame and body temperature was maintained at $37^{\circ} \mathrm{C}$ and monitored by using a rectal thermometer. Electrocardiograph leads were then attached at the left foreleg and right hind leg to monitor the heart rate 
continuously throughout the experiment. In case of a monocular deprivation, the deprived eye was re-opened by cutting the surgical knots and removing all sutures carefully. The eye was then cautiously flushed and drying was prevented by applying eye drops (Corneregel fluid, Bausch \& Lomb, Berlin, Germany). Finally, both eyes were swabbed with silicon oil (Carl Roth, Karlsruhe, Germany) and covered with aluminum foil to prevent exposure to light and drying.

The head and neck was shaved thoroughly to remove the fur, disinfected using $70 \%$ ethanol (Carl Roth, Karlsruhe, Germany) and locally anesthetized with xylocaine gel (reactive agent lidocaine hydrochloride, AstraZeneca, Wedel, Germany). Subsequently, the scalp and underlying connective tissue was removed, thereby carefully exposing the skull which was rinsed with 0.9 \% sodium chloride solution (Sigma - Aldrich, Steinheim, Germany). The skull was then covered with a wet pad soaked in saline before hand - hot, fluidized agarose gel ( 2.5 \%; Biomol, Hamburg, Germany) filled in a syringe was applied on the skull to cover the area above the visual cortex. Before complete re - solidification of the agarose, a rectangular glass cover slip was placed above the occipital lobe of the left hemisphere. Additionally, agarose gel was applied around all sides of the cover slip to ensure that the region of interest was fully covered. To prevent the skull from drying - out, the glass cover slip and agarose gel were lined with Vaseline. A light-tight, black cardboard crown was placed on the head of the mouse between the eyes and the exposed skull to protect the eyes from light coming from the cold light source which was used in the experiment (Figure 12). 


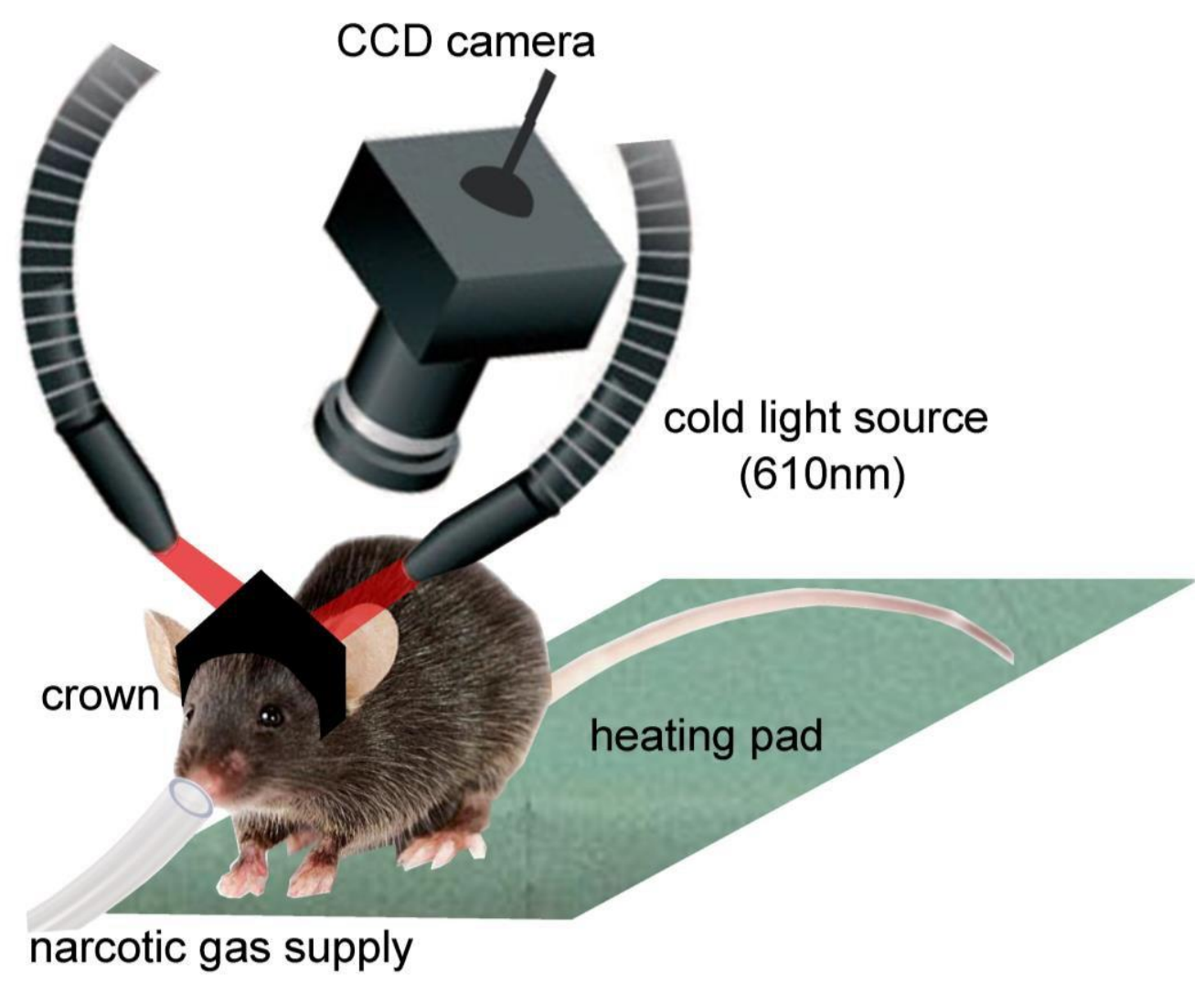

Figure 12: Schematic overview of the surgery setup. The mouse is anesthetized via narcotic gas supply through a tube attached to the nose and sitting on a heating pad, thereby keeping the body temperature constant at 37 ${ }^{\circ} \mathrm{C}$. A cold light source with a wavelength of $610 \mathrm{~nm}$ is illuminating the skull over the visual cortex and visual cortical activity (light absorption of deoxyhemoglobin) is captured with a CCD - camera. A black crown positioned on the head is protecting the eyes from red light exposure.

\subsubsection{Acquisition of intrinsic signals}

Mouse visual cortical responses were recorded using in vivo optical imaging of intrinsic signals (Blasdel \& Salama, 1986; Grinvald et al., 1986; Bonhoeffer and Grinvald, 1993), which has been further optimized to analyze OD-plasticity by Kalatsky and Stryker (2003). The intrinsic signals obtained from the visual cortex primarily result from a hemodynamic shift due to an increased neuronal activity in response to appropriate visual stimulation. Active neurons require oxygen, resulting in a temporarily increased level of deoxyhemoglobin in the blood of the surrounding vasculature. The wavelength of light absorption of oxygenated and deoxygenated hemoglobin differs from each other (Figure 13), a property which can be used to visualize changes in neuronal activity in response to visual stimulation in vivo. 


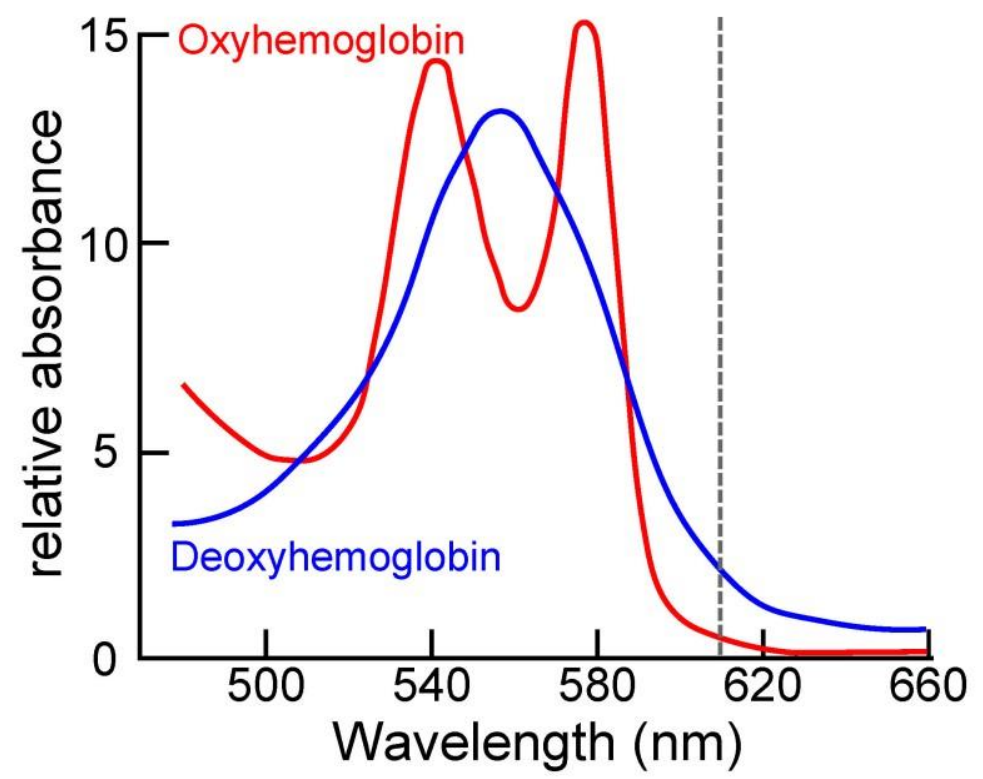

Figure 13: A schematic overview of the absorption spectra of oxyhemoglobin $\left(\mathrm{HbO}_{2}\right)$ in red and deoxyhemoglobin $(\mathrm{Hbr})$ in blue displaying the choices of wavelengths of filters $(610 \mathrm{~nm})$ for the illumination of V1 used for optical imaging of intrinsic signals.

To identify the exact position of the visual cortex beneath the skull, it is required to focus the CCD camera which converts light into electrical signals (Dalsa 1M30, Dalsa, Waterloo, Canada) on the local vasculature above the brain. To this end, the left hemisphere around the V1 was illuminated with green cold light of 550 nm (KL 2500 LCD, Zeiss, Jena, Germany). An interference filter ( $550 \pm 10 \mathrm{~nm}$ ) was placed between the camera and the objective to prevent light scattering. We imaged a cortical area of $4.6 \times 4.6 \mathrm{~mm} 2$ and the reflected light was then recorded with the camera equipped with two $135 \times 50$ mm lenses (Nikon, Melville, New York, USA) arranged in a tandem manner and positioned in parallel $\left(25^{\circ}\right.$ rotated rightwards and $7^{\circ}$ backwards) to the visual cortical surface. A picture of the V1 surface vasculature was taken to verify the focus and position of the camera. Subsequently, the focal plane of the camera was lowered by $600 \mathrm{~nm}$ which is corresponding to the visual cortical layer IV from which we record V1 activation. To finally visualize intrinsic signals, V1 was illuminated with cold red light with a wavelength of $610 \mathrm{~nm}$ and a respective interference filter was placed between the camera and the objective to prevent light scattering of inappropriate wavelengths that could otherwise also be absorbed by deoxygenated hemoglobin. 


\subsubsection{Acquisition of phase and activity maps}

The mouse visual cortex consists of a binocular and a monocular region. While in the monocular region, inputs from the contralateral eye are processed exclusively, inputs from both eyes are processed in the binocular part. To determine the degree of ocular dominance, it is therefore important that only the binocular zone is stimulated to prevent overestimation of contralateral inputs resulting from partial co-stimulation of the monocular part of the V1. A 24 -inch LCD monitor screen (BL2400PT, Benq, Taipeh, Taiwan) was placed in front of the mouse in a distance of $25 \mathrm{~cm}$ and with the vertical midline of the screen in line with the mouse. A white bar measuring $2^{\circ}$ in height and $20^{\circ}$ in width with regard to the animal's visual field served as stimulus and was displayed on black screen background. Defining the midline of the monitor screen as $0^{\circ}$, the stimulus was positioned between $-5^{\circ}$ (left hand from the midline) and $15^{\circ}$ (right - hand from the midline). As can be seen in Figure 14, measures and position of the stimulus were chosen such that only the binocular region of the left V1 was visually stimulated. 


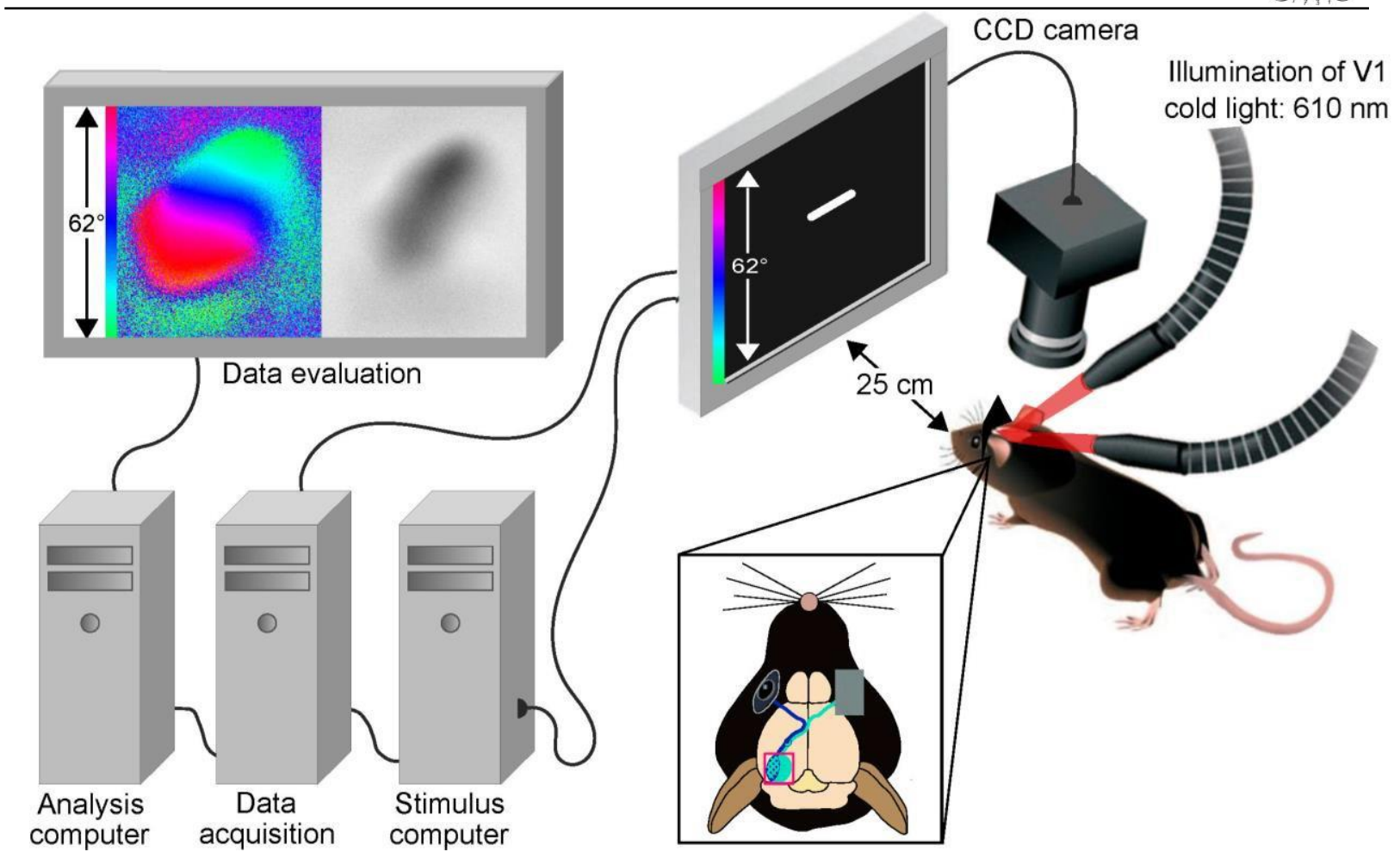

Figure 14: Schematic overview of the setup used for optical imaging of intrinsic signals in vivo. A $20^{\circ}$ wide horizontally moving white bar is projected on a computer monitor positioned $25 \mathrm{~cm}$ in front of the mouse which serves as a stimulus merely activating the binocular region of the left visual cortex. The resulting hemodynamic

shift in response to this stimulus is visualized using illumination of the visual cortical area with cold red light with a wavelength of $610 \mathrm{~nm}$. The reflection of the light is then recorded with a CCD camera transmitting the data to an acquisition computer. Using an analysis computer, the data are subsequently analyzed to depict cortical maps and calculate the ocular dominance index.

The stimulus used was programmed to move horizontally either up- or downwards with a velocity of $10^{\circ} / \mathrm{s}$ within a screen section of $80^{\circ}$, resulting in a period of 8 seconds. Between the two stimulus periods, there is no delay, resulting in a continuous stimulus passing the defined screen section every 8 seconds. The software ContStim (VK Imaging, Houston, Texas, USA) was used to display the stimulus. Choosing an appropriate stimulus frequency is of major importance to prevent the acquisition of intrinsic signals from being biased by background noise such as heart rate, respiration or vasomotor pulses. The stimulus frequency was determined accordingly to minimize interference such that remaining background noise was excluded from the intrinsic signals via Fourier analysis. In addition, the experiment was performed in darkness and as the non - stimulated eye was covered with aluminum foil, the measured activity changes in the $\mathrm{V} 1$ almost exclusively represented the response to the 
stimulus of the single eye. The stimulus was alternately presented to each eye respectively, first moving upwards $\left(90^{\circ}\right)$ then downwards $\left(270^{\circ}\right)$, while the other eye was covered with aluminum foil. Frames were acquired at a rate of $30 \mathrm{Hertz}$ temporally and then binned to 7.5 Hertz and stored as $512 \times 512$-pixel images after spatial binning of the camera image.

Eye drops (Corneregel fluid, Bausch \& Lomb, Berlin, Germany) were repeatedly applied throughout the experiment to prevent the eyes from drying out. The measured hemodynamic shift occurred with a certain delay (Hemodynamic delay; $\phi D)$ to the respective stimulation corresponding to increased neuronal activity. The delay was relativized by integrating the cortical maps from stimulation with both upward - and downward - moving stimuli. These integrated maps are referred to as absolute cortical maps. Under the stimulus pattern and conditions described here, it was possible to acquire cortical maps for both phase and activity.

\subsection{Visual stimuli}

\subsubsection{Acquisition of map quality from elevation and azimuth maps}

To acquire map quality and cortical maps from both, the monocular and the binocular region of the left V1, the monitor screen was positioned with an angle of $45^{\circ}$ to the right eye of the mouse. The left eye was covered with aluminum foil and the experiment was performed in darkness. The distance between the screen and the right eye of the mouse was $25 \mathrm{~cm}$ and the midline of the screen was aligned to the mouse (Figure 15). A white horizontal bar (width $94^{\circ}$, height $\left.2^{\circ}\right)$ moving either from left to right $\left(180^{\circ}\right)$ or from right to left $\left(0^{\circ}\right)$, as well as a horizontal bar (width $2^{\circ}$, height $\left.62^{\circ}\right)$ moving either upwards $\left(90^{\circ}\right)$ or downwards $\left(270^{\circ}\right)$ served as a stimulus to acquire cortical maps from the binocular and monocular region of the left hemispherical V1, which could be used to determine map quality. To test maximum response and map quality, the drifting bars were shown across the full screen $\left(94^{\circ}\right.$ azimuth and $62^{\circ}$ elevation, respectively). Therefore, both stimuli (either horizontal or vertical direction) were presented to the right eye with the following order: $90^{\circ}, 270^{\circ}, 0^{\circ}, 180^{\circ}$ and each run lasted for five minutes. The bars were $2^{\circ}$ wide, corresponding to a spatial frequency of $0.25 \mathrm{cyc} / \mathrm{deg}$ with a drifting speed of $10 \%$ second and appeared at a rate of 0.125 Hertz The maps acquired by displaying visual stimulation of V1 are referred to as azimuth maps (for vertical moving bars) and elevation maps for horizontally moving bars. 

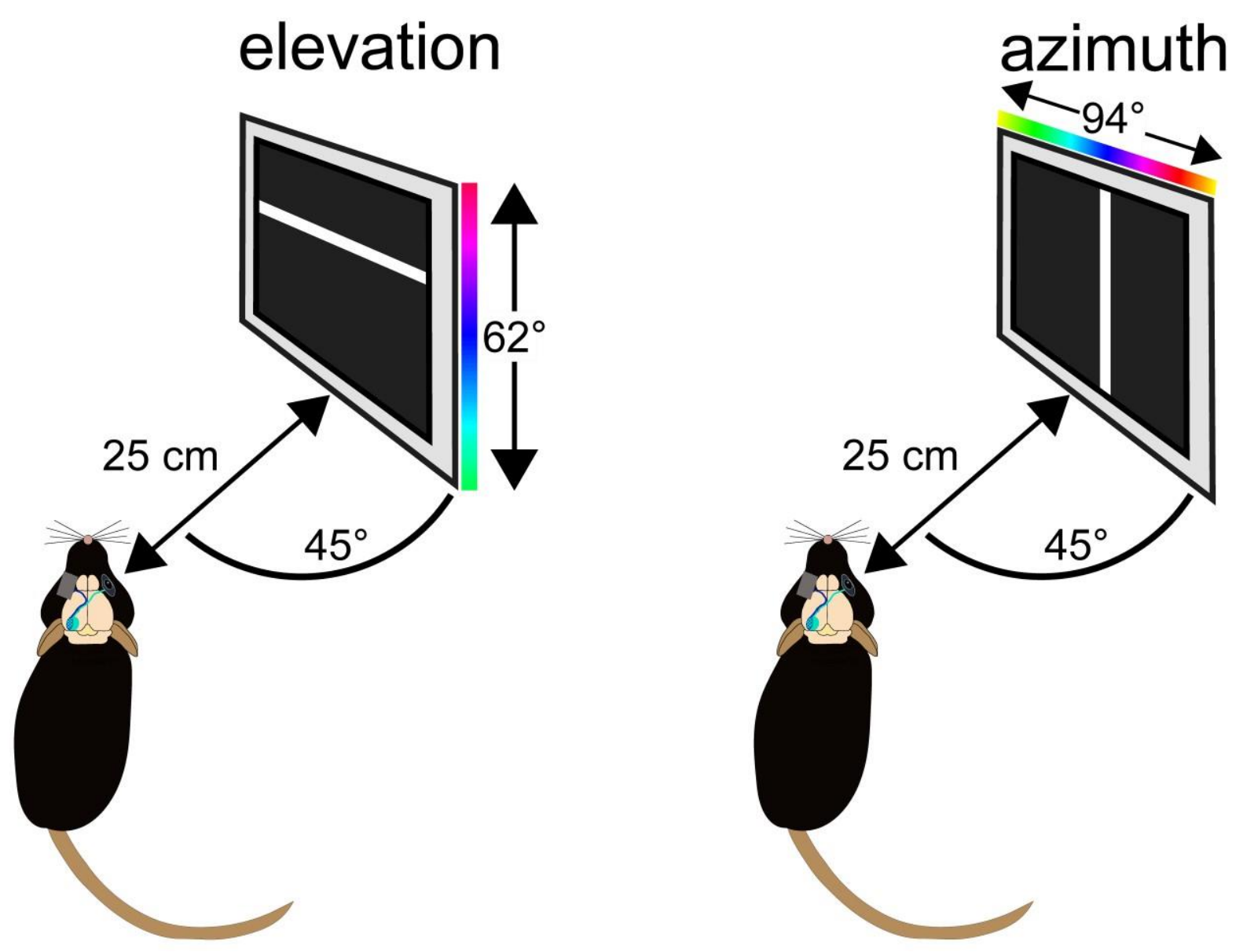

Figure 15: Position of the stimulus monitor and visual stimuli. For stimulating the visual cortex of the left hemisphere as much as possible, the monitor screen was positioned $25 \mathrm{~cm}$ in front of the mouse's right eye with an angle of $45^{\circ}$. Visual stimuli to measure maximum response and map quality of elevation and azimuth maps. The stimulus consists of a white moving bar of $2^{\circ}$. The monitor had a visual angle of $62^{\circ}$ height and $94^{\circ}$ width. The position of the stimulus within the visual field was color-coded to test for retinotopy.

In respect to the hemodynamic delay (of about one to two seconds), the resulting map of one direction (for example $90^{\circ} \uparrow$ ) was only a map of relative retinotopy (Cang et al., 2005b). Therefore, maps of absolute retinotopy were computed by reversing the stimulus $\left(270^{\circ} \downarrow\right)$, which leads to the reversal of the response sequence in which the delay is the same. The software subtracted the response time to the reversed stimulus $\left(270^{\circ} \downarrow\right)$ from the response time to the direct one $\left(90^{\circ} \uparrow\right)$ and computed an absolute response map without the hemodynamic delay (Cang et al., 2005b). The same computation was done for maps resulting from the azimuth orientation respectively $\left(0^{\circ} \rightarrow\right.$ and $\left.180^{\circ} \leftarrow\right)$. 


\subsection{Data Analysis}

\subsubsection{Maximum cortical activation and map quality}

Recorded maps from the CCD camera were calculated from the acquired frames using Fourier analysis to extract the signal at the stimulation frequency using the custom software iman (image analysis; VK Imaging, Houston, Texas) and saved by the software ContImage (VK imaging, Houston, Texas). The area of the visual cortex was cropped to $300 \times 300$ pixels with mapans (map analysis single; VK Imaging, Houston, Texas) and the signal of the activated region was normalized with respect to its surrounding background. Maps acquired with the stimulus bar moving up and down (elevation orientation) as well as left and right (azimuth orientation) were combined by mapanm (map analysis multiple; VK Imaging, Houston, Texas) to an average map to correct for the hemodynamic delay. The color - coded retinotopic phase map encodes the visually activated region in the V1 by referring to the position of the stimulus bar on the stimulus monitor. The phase component of the signal is used for the calculation of retinotopy (retinotopic phase map, Figure 16).

To create V1 activity maps, the reduction in light reflectance as a result from the conversion from oxy - to deoxyhemoglobin was used and hence the hemodynamic shift as an indirect indicator for neuronal activation. The higher the hemodynamic shift in response to visual stimulation, the higher is the change in light reflectance of V1. The amplitude component represents the intensity of visual cortical activation expressed as fractional change in reflectance $\times 10^{-4}$ (raw magnitude response map, Figure 16). The strongest pixel within the selected area of the visual cortex is set as the value of maximum response $\left(\times 10^{-4}\right)$ (Figure 16). 


\section{elevation}

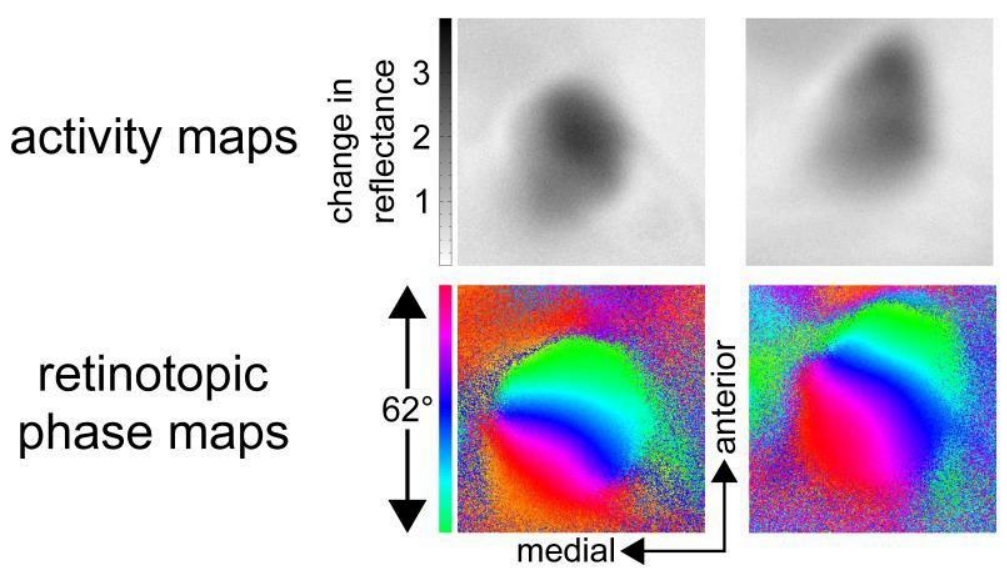

\section{azimuth}

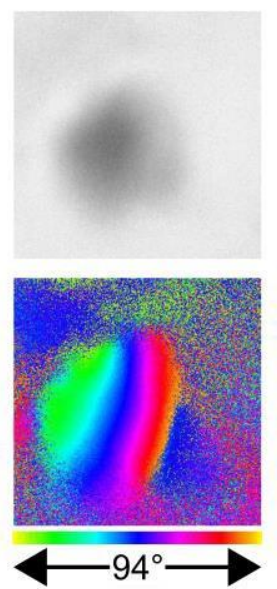

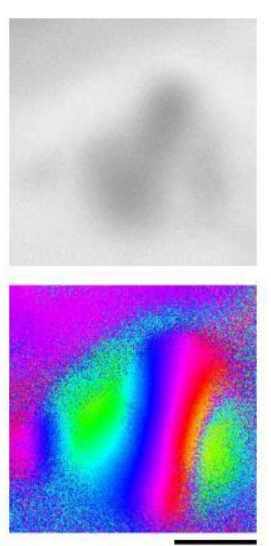

Figure 16: Maps of the left visual cortex after visual stimulation with a horizontal or vertical moving bar respectively; acquired by optical imaging of intrinsic signals. Upper row: Raw magnitude response maps which indicate the activity of the visual cortex. The active area is visualized by dark colored pixel, the darker the pixel, the higher is the maximum response. The maximum response is specified as a change in reflectance $\mathrm{x} 10^{-4}$. Bottom row: Retinotopic phase maps which are color coded according to the position (in degree) of the bar on the monitor screen. Scale bar: $1 \mathrm{~mm}$.

To assess the quality of retinotopic maps, we used the calculation introduced by Cang (Cang et al., 2005a) by using the software MATLAB (The MathWorks, Natick, Massachusetts, USA). Both the elevation and azimuth maps were used to select the most responsive 20.000 pixels in the visual cortex. For every pixel, the difference between its position and the mean position of its 24 surrounding pixels was calculated. For maps of high quality, the position differences are very small based on smooth progression. The standard deviation of the position difference was then used as an index of the quality of retinotopic maps, with small values indicating high map quality and high values indicating low map quality (Cang et al., 2005b).

\subsection{Ocular dominance index and visual cortical maps}

The ocular dominance index (ODI) serves as a measure for ocular dominance which is important for testing OD-plasticity, since the binocular part of the V1 is usually dominated by inputs after visual stimulation of the contralateral eye. Monocular deprivation can induce a shift of V1 activation towards the ipsilateral eye to what we refer to as ocular dominance (OD) plasticity. To quantify OD-plasticity, an OD-score of each pixel in the binocularly activated region was calculated. Since both eyes of the mouse are stimulated separately in the experiment, one can define cortical activation measured for both eyes independently and 
calculate the ocular dominance index (ODI) accordingly with the following formula:

$$
O D I=\frac{\mathrm{C}-\mathrm{I}}{\mathrm{C}+\mathrm{I}}
$$

In which $\mathrm{C}$ and I refer to the cortical activation after visual stimulation of either the contra (C) or the ipsilateral eye (I), respectively. The ODI values can range between -1 and +1 , with positive values indicating contralateral dominance and negative values indicating ipsilateral dominance, whereas 0 represents an equilibrium between the cortical activation induced by contra - or ipsilateral eye inputs. ODIs from blocks of four runs were calculated, in which the averaged maps for each eye had at least a response magnitude of $1.0 \times 10-4$. Typically, we obtained three to six ODIs per animal which were averaged for further quantification and data display with MATLAB (averagemaps, written by Dr. Simon Gengler). The ODIs were color - coded in a 2 - dimensional map of the OD scores (OD map) with cold, blue colors representing negative values (ipsilateral eye dominance), and warm, colors representing positive values (contralateral eye dominance) (Figure 17).

A

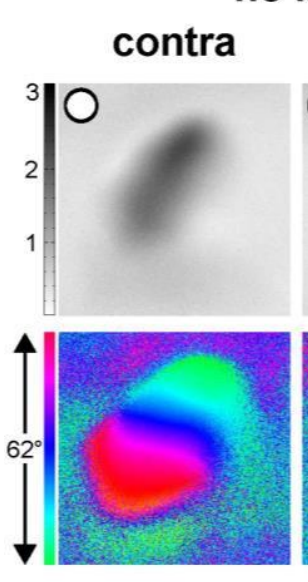

no MD

\section{ipsi}
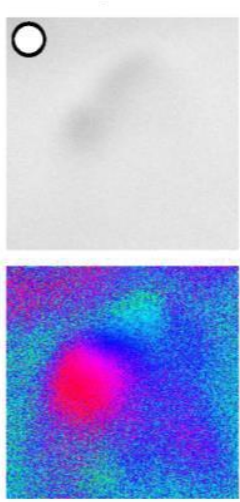

B

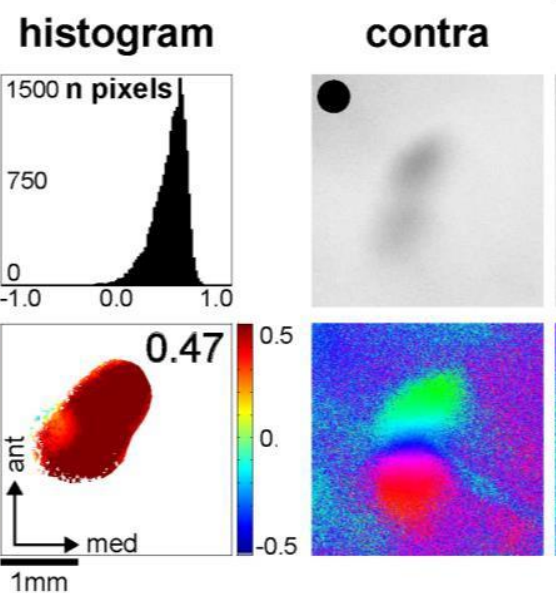

after 4 days of MD ipsi
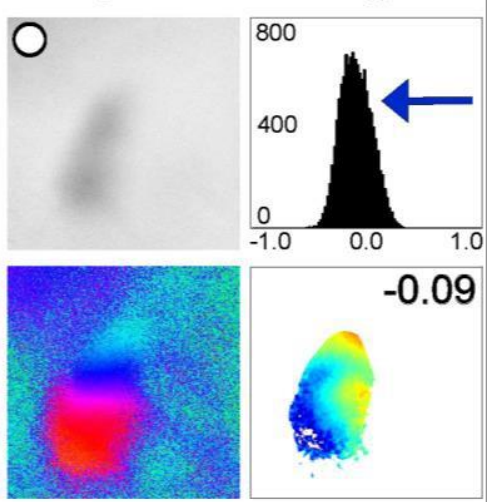

histogram
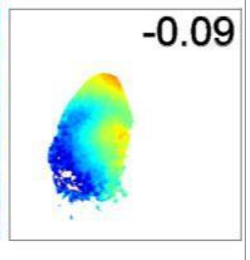

Figure 17: Quantification of the activity, phase and color coded ocular dominance maps and the histograms of the OD index. (A) The maximum response maps of the left visual cortex after visual stimulation (with a $20^{\circ}$ horizontal bar) of the contralateral (left) and the ipsilateral (right) eye, respectively. The response is restricted to the binocular zone of V1. White circles indicate that the respective eye remained open whereas black circles indicate monocular deprivation 4 days prior to optical imaging of the respective eye. The greyscale bar with numbers indicates the maximum response in change in reflectance $\times 10^{-4}$ in V1 induced by either contra- or ipsilateral eye stimulation. Second row: phase maps color coded with respect to the location of the moving 
stimulus bar on the according position on the monitor screen. (B) Histogram of the single OD scores and their distribution in number of pixels. The ocular dominance index, the average of all single OD-scores calculated from the maximum response maps of contra $(C)$ and ipsi $(I)$ are shown in the left corner of the histogram. OD-map: The calculation of OD scores for every pixel where the active region is defined as the cortical area within every pixel magnitude is at least $30 \%$ of that of the pixel with the maximum response magnitude. The pixels are colorcoded while cold colored pixel (e.g. blue, green) represent an ocular dominance in the binocular zone of the visual cortex of the ipsilateral eye, whereas warm colored pixel (e.g. yellow, red) represent an ocular dominance of the contralateral eye, respectively. (Abbreviations: $\mathrm{OD}=$ ocular dominance, contra / $\mathrm{C}=$ contralateral eye, ipsi / I = ipsilateral eye). Scale bar: $1 \mathrm{~mm}$.

\subsubsection{AAV transduction in $P 0$ mice}

For adeno - associated virus (AAV) delivery to knock down PSD-95 or PSD-93 in the visual cortex, P 0-P 2 mouse pups were anesthetized on ice for about four minutes and immobilized with a holder on the injection table which surface temperature was kept constant at $4{ }^{\circ} \mathrm{C}$. The intersection of the sagittal and the lambdoid suture (lambda) was visually identified through the translucent skin. Injections of AAV (13.4 $\mathrm{nL}$ each, $23 \mathrm{~nL} / \mathrm{s})$ were delivered at two positions bilaterally into V1 (from lambda, in $\mathrm{mm}: \mathrm{AP}+0.1, \mathrm{ML} \pm 1.25$, $\mathrm{DV}-0.8$ and $\mathrm{AP}+0.3, \mathrm{ML} \pm 1.8$, DV - 0.8) using a glass capillary with a Nanoject II microinjector (Drummond) (Figure 18). Afterwards, the pups were transferred to a heating plate for recovery and returned to their home cage. 


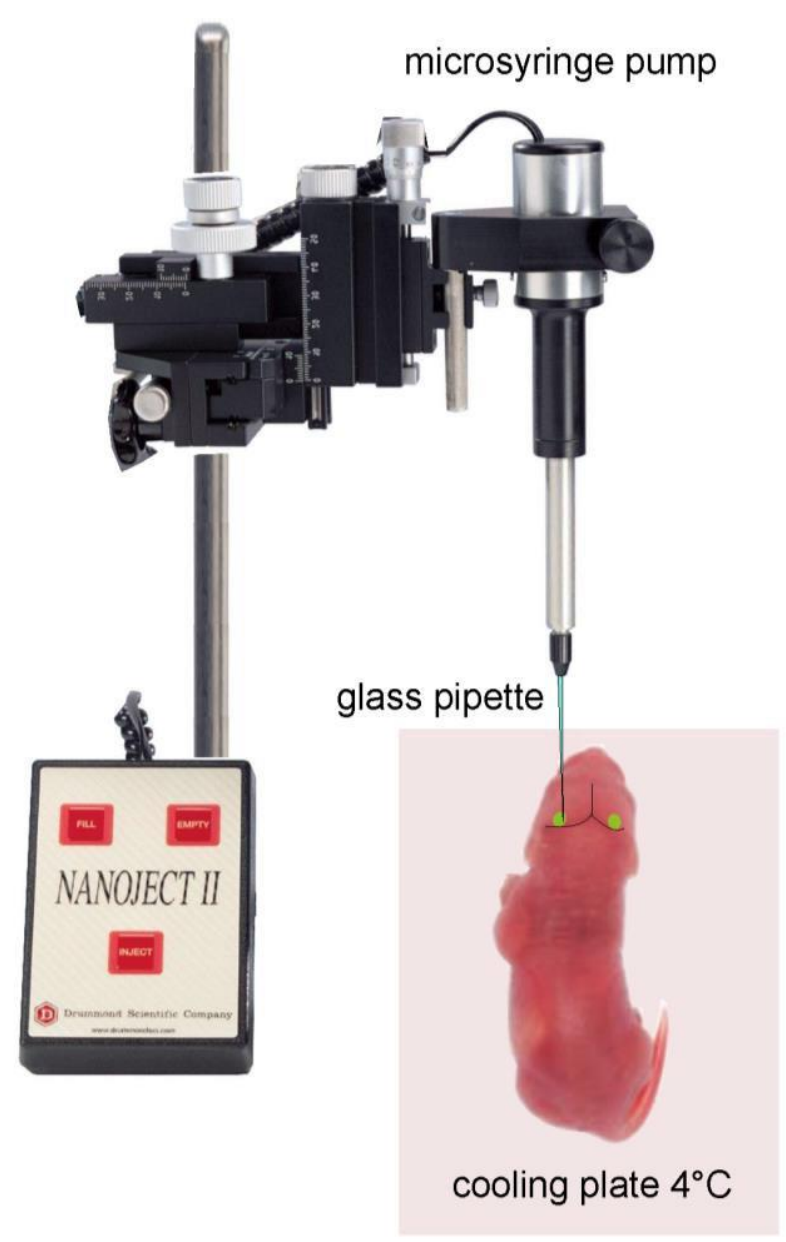

Figure 18: Schematic representation of the virus injection setup for mouse pups at P 0 - P 2: The mouse is cooled down on ice and placed on a cooling plate with a constant temperature of $4^{\circ} \mathrm{C}$. The virus is injected into the V1 of both hemispheres at two different injections sites per hemispheres with a micro syringe pump using a glass pipette.

\subsubsection{Adult virus injection}

For virus injections in adult mice (> P 40, P 143), animals were deeply anesthetized with a mixture of fentanyl $(0.07 \mathrm{mg} / \mathrm{kg})$, midazolam $(7.0 \mathrm{mg} / \mathrm{kg})$, and medetomidine $(0.7 \mathrm{mg} / \mathrm{kg})$. Fentanyl is a potent, synthetic opioid analgesic with a rapid onset and short duration of action and is commonly used as a pain reliever as well as an anesthetic in combination with benzodiazepines like midazolam, a short - acting benzodiazepine enhancing the effect of gamma-Aminobutyric acid (GABA) on GABAA receptors resulting in neural inhibition and sedation (Olkkola et al., 2008). Medetomidine is a synthetic drug commonly used as a surgical anesthetic. After general anesthesia, the animal was placed on a heating pad to prevent cooling and the head was fixed with a stereotaxic apparatus (Stoelting) and the eyes were protected from drying out with Bepanthen salve (Roche) and covered with aluminum foil to 
protect from light exposure. The skin over the visual cortex was cleaned with $70 \%$ ethanol and carefully pushed aside. In each hemisphere, two holes were drilled into the skull with following directions from Bregma in $\mathrm{mm}$ : $\mathrm{AP}-3.8, \mathrm{ML} \pm 2.4$, $\mathrm{DV}-1.6$ and $\mathrm{AP}-2.8, \mathrm{ML} \pm 3.0$, DV - 1.4. According to the positions of the wholes, $0.5 \mu \mathrm{AAV}(100 \mathrm{~nL} / \mathrm{min})$ was injected at two positions bilaterally into V1 (from Bregma, in $\mathrm{mm}$ : AP - 3.8, ML \pm 2.4 , DV - 1.6 and AP 2.8, $\mathrm{ML} \pm 3.0, \mathrm{DV}-1.4)$ using a glass pipette controlled by a micro syringe pump controller (WPI) (Figure 19).

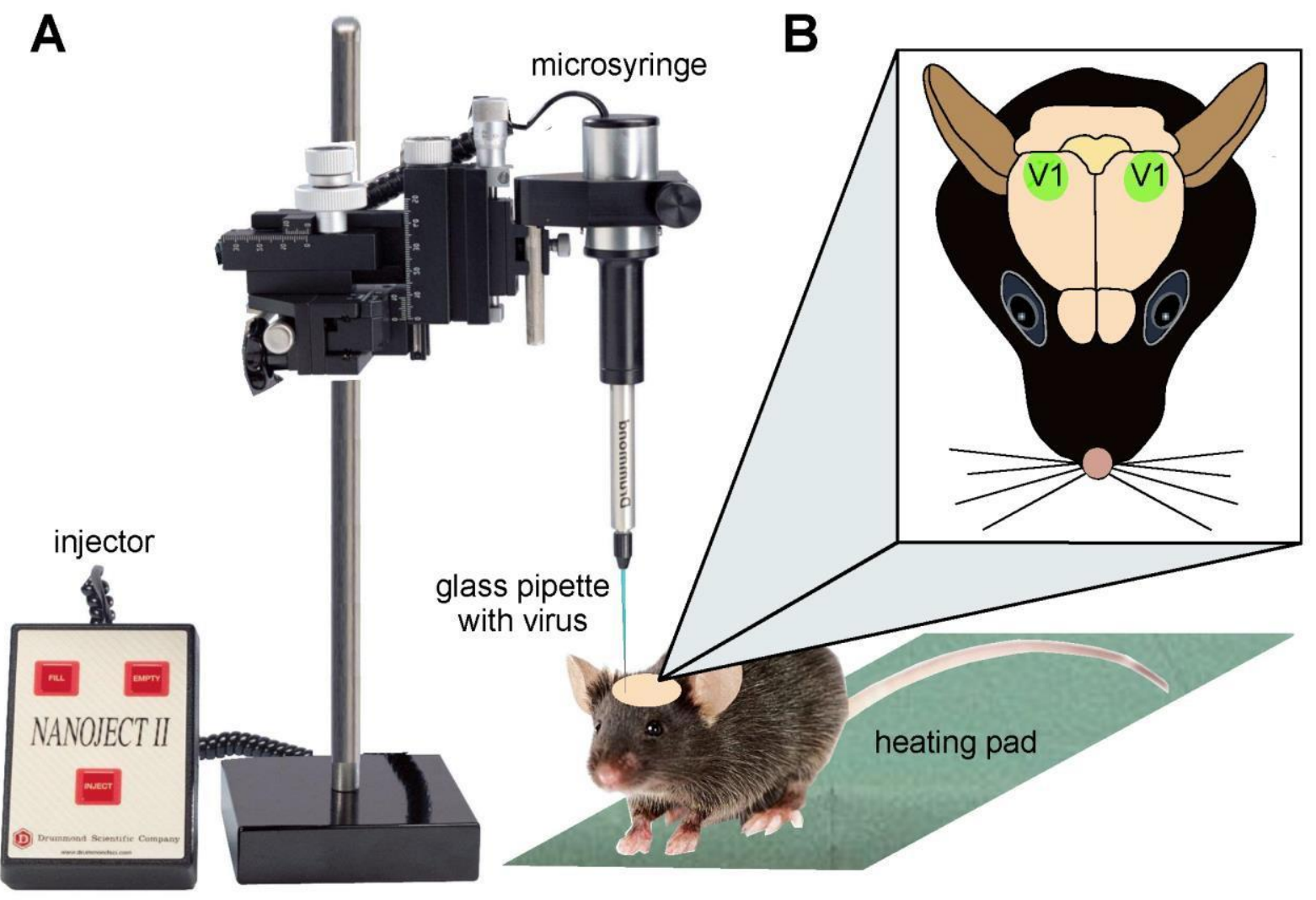

Figure 19: Schematic representation of the virus injection setup: (A) The mouse is deeply anesthetized and placed on a heating pad. The head is fixed with a stereotactic apparatus and the virus is injected into the V1 of both hemispheres at two different injections sites per hemispheres with a micro syringe pump using a glass pipette. (B) The position of the injected virus in the visual cortex of both hemispheres.

Afterwards, the skin was sutured back using 4 - 0 silk (Ethicon, Norderstedt) and covered with Braunovidon salve (active component: Povidone - iodine, B Braun, Melsungen, Germany) to prevent inflammation. The anesthesia was then reversed using atipamezole $(2.5 \mathrm{ml} / \mathrm{kg}), \mathrm{a}$ synthetic $\alpha 2$ adrenergic receptor antagonist used for reversing the sedative and analgesic 
effects of medetomidine. To prevent pain, Rimadyl ( $5 \mu \mathrm{g} / \mathrm{g}$ mouse; active agent: Carprofen; Pfizer, Berlin) and buprenorphine ( $4.25 \mathrm{ml} / \mathrm{kg})$, which is a semisynthetic opioid derivative of thebaine, were injected. Finally, the animal was returned to the home cage and provided with a heating lamp until it fully recovered from anesthesia.

\subsection{Perfusion}

Mice were perfused and the brains removed after the optical imaging experiments. Mice were injected with an i.p. injection of $0.3 \mathrm{ml}$ chloral hydrate (Sigma Aldrich, Steinheim, Germany) while still being under deep general anesthesia. Subsequently, they were transferred to a Styrofoam plate and fixated. The thoracic cavity was opened and the heart carefully exposed. A cannula was inserted into the left ventricle of the heart and the right atrium cut open. With a perfusion pump (MC - MS CA8 / 6, Ismatec, Glattbrugg-Zürich, Switzerland), the mouse was perfused with $50 \mathrm{ml} 0.9 \%$ saline containing $0.05 \mathrm{ml}$ heparin for two minutes with $25 \mathrm{ml} / \mathrm{min}$ to flush the blood out of the body. Afterwards, the tissue was fixated using $75 \mathrm{ml}$ of $4 \%$ paraformaldehyde (PFA) for 3 minutes with $25 \mathrm{ml} / \mathrm{min}$. The brain was then carefully removed and stored in a falcon tube with $4 \%$ PFA at $4{ }^{\circ} \mathrm{C}$ overnight. On the following day, the brains were transferred to phosphate buffer saline (PBS) and stored at $4{ }^{\circ} \mathrm{C}$ until further analysis.

\subsection{Confocal image analysis of viral transfected brain slices}

Brains of mice that had obtained an AAV - sh95 mediated knockdown of PSD-95 or an injection of AAV - shlc in the visual cortex at P $0-P 2$ or P 40, were further analyzed for transfection efficiency of the respective virus. As GFP is bicistronically expressed by the construct, its fluorescence can provide information about the general success of the virus transfection. Brains were sliced $(300 \mu \mathrm{m})$ and prepared in PBS by using a vibratome including V1. After three incubations ( $1 \mathrm{~min}$ ) in PBS, brain slices were incubated with $3 \mu \mathrm{g} / \mathrm{ml}$ DAPI for 10 minutes. After an additional PBS incubation, they were mounted on glass slides and covered with glass cover slips. After drying, they were cleaned with ethanol and confocal images were acquired with a Carl Zeiss LSM 710 microscope using a $10 \times$ objective and a resolution of 2.048 $\times 2.048$ pixels. Imaging parameters were maintained constant for the acquisition of different conditions throughout the experiments. 


\subsection{Analysis of virus transfected brain slices with a fluorescent microscope}

Brains of mice that had obtained either an AAV - sh95 mediated knockdown of PSD-95 in the visual cortex of both hemispheres or an injection of AAV - shlc at > P 110 or P 0 injections of AAV - sh93 mediated knockdown of PSD-93 were further analyzed for the transfection efficiency of the respective virus. Brains were cut with a vibratome in $200 \mu \mathrm{m}$ thick slices and mounted on a glass slide. Images from coronal brain sections were acquired with a Nikon SMZ18 research stereomicroscope using a wide-field fluorescence microscope excitation light source (X-Cite 120Q) with a 120-watt lamp to deliver rich spectral excitation energy and uniform wide-field fluorescence illumination to the sample plane (Excelitas Technologies Corp.). Images were taken with AxioCam HSm (Carl Zeiss) camera. Imaging parameters were maintained constant for the acquisition throughout the experiments.

\subsection{Statistical analysis}

Statistical analyses were done using GraphPad Prism (version 5.04). All intra - and intergroup comparisons were analyzed by a two - tailed Student $\mathrm{t}$ - test (with Bonferroni correction). The intergroup comparison of the enhancement of "visual acuity" and "contrast sensitivity" were analyzed by ANOVA with repeated measurements and Bonferroni correction. The levels of significance were set as $* p<0.05 ; * *<0.01 ; * * p<0.001$. Data are represented as means \pm SEM. 


\subsection{Solutions of ingredients used and recipes}

Table 6:

\begin{tabular}{|c|c|}
\hline Agarose $(2.5 \%)$ in saline & $\begin{array}{c}5 \text { g agarose mixed with } 200 \mathrm{ml} 0.9 \% \text { saline } \\
\text { boil and wait until suspension is } \\
\text { transparent fill in glass containers and } \\
\text { store in fridge }\end{array}$ \\
\hline Chloral hydrate & $\begin{array}{c}30 \mathrm{~g} \text { chloral hydrate in } 100 \mathrm{ml} \text { distilled } \\
\text { water }\end{array}$ \\
\hline Chlorprothixene & $\begin{array}{l}4 \mathrm{mg} \text { chlorprothixene mixed with } 1 \mathrm{ml} \\
\text { distilled water }\end{array}$ \\
\hline $\begin{array}{l}\text { Phosphate buffer in PB (PBS); } 0.1 \text { molar; } \mathrm{pH} \\
\text { 7.4) }\end{array}$ & $\begin{array}{c}\operatorname{mix} 3.75 \mathrm{~g} \mathrm{NaH}_{2} \mathrm{PO}_{4} \times 2 \mathrm{H}_{2} \mathrm{O} \\
9.75 \mathrm{~g} \mathrm{Na}_{2} \mathrm{HPO}_{4} \text { with distilled water to a } \\
\text { total volume of } 1 \text { liter }\end{array}$ \\
\hline Paraformaldehyde (PFA 4 \%; pH 7.4) & $\begin{array}{l}40 \mathrm{~g} \text { paraformaldehyde mixed in } 300 \mathrm{ml} \text { of } \\
\text { distilled water heated at } 60^{\circ} \mathrm{C} \text { until } \\
\text { suspension is translucent } \\
\text { add } 6-8 \text { drops of } 10 \mathrm{~m} \mathrm{NaOH} \text { filter solution } \\
\text { and adjust } \mathrm{pH} \text { to } 7.4 \\
\text { let solution cool down and add distilled } \\
\text { water to a total volume of } 1 \text { liter }\end{array}$ \\
\hline saline $(\mathrm{NaCl} ; 0.9 \%, \mathrm{pH} 7.0)$ & $0.9 \mathrm{~g}$ of $\mathrm{NaCl}$ in 1 liter distilled water \\
\hline Heparin in $0.9 \%$ saline & $\begin{array}{c}53.33 \mathrm{mg} \text { heparin (25.000 i.u.) in } 0.9 \mathrm{~g} \mathrm{NaCl} \\
\text { mixed in } 1 \text { liter distilled water }\end{array}$ \\
\hline
\end{tabular}




\begin{tabular}{|c|c|}
\hline Agarose & Biomol (1280) \\
\hline \multicolumn{2}{|l|}{ Atipamezole } \\
\hline Atropinsulfat (atropine sulfate) & $\begin{array}{l}\text { Dr. Franz Köhler Chemie (PZN: 1821288), } \\
\text { Benstein }\end{array}$ \\
\hline Braunovidon Salve (Povidone-iodine) & BBraun, Melsungen \\
\hline \multicolumn{2}{|l|}{ Buprenorphine } \\
\hline Chloral hydrate & Sigma (15307) \\
\hline Chlorprothixene hydrochloride & Sigma (C1671) \\
\hline Cornerege $\left.\right|^{\circledR}$ Fluid (eye drops) & Bausch \& Lomb, Berlin \\
\hline Dexa-ratiopharm ${ }^{\circledR}$ (dexamethasone) & ratiopharm GmbH (PZN: 7720996) \\
\hline di-Sodium hydrogen phosphate (Na2HPO4) & Roth (P030.2) \\
\hline Ethanol $70 \%$ denatured & Roth (T913.3) \\
\hline Fentanyl & Pfizer \\
\hline $\begin{array}{l}\text { Fortecortin } \\
\text { (dexametasondihydrogenphosphate- } \\
\text { dinatrium }\end{array}$ & Merck Serono, Darmstadt \\
\hline halothane & Sigma (B4388) \\
\hline Heparin (25.000 I.E.) & Rotexmedica GmbH (PZN: 3862340) \\
\hline Paraformaldehyde (PFA) & Roth (0335.3) \\
\hline gentamycin (gentamicin sulfate) & medphano, Berlin \\
\hline \multicolumn{2}{|l|}{ Medetomidin } \\
\hline \multicolumn{2}{|l|}{ Midazolam } \\
\hline Rimady $\left.\right|^{\circledR}$ (Carprofen) & $\begin{array}{c}\text { Pfizer (approval number: 400684.00.00), } \\
\text { Berlin }\end{array}$ \\
\hline saline ( $\mathrm{NaCl}, 0.9 \%$ solution for injection) & B. Braun Melsungen AG \\
\hline
\end{tabular}




\begin{tabular}{|c|c|}
\hline Silicone oil M 20.000 & Roth (4060.1) \\
\hline Sodium chloride (NaCl) & Roth (3957.1) \\
\hline $\begin{array}{c}\text { Sodium dihydrogen phosphate dehydrate } \\
(\mathrm{NaH} 2 \mathrm{PO} 4 \times 2 \mathrm{H} 2 \mathrm{O})\end{array}$ & Roth (T879.2) \\
\hline sodium hydroxide (NaOH) & VWR (28.244.262) \\
\hline $\begin{array}{c}\text { Xylocain }{ }^{\oplus} \mathrm{Gel} 2 \%(\text { lidocaine hydrochloride } \\
\left.\mathrm{H}_{2} \mathrm{O}\right)\end{array}$ & AstraZeneca GmbH, Wedel \\
\hline
\end{tabular}




\section{Results}

\subsection{The juvenile form of OD-plasticity is preserved in PSD-95 KO mice until late adulthood}

Experience-dependent cortical plasticity is typically tested with ocular dominance (OD) plasticity (OD-plasticity) in the primary visual cortex (V1), which can be induced by monocular deprivation (MD). In the binocular region of the mouse V1, neurons respond to sensory inputs coming from both eyes, but visual cortical activation is dominated by afferents from the contralateral (contra) eye with only little input coming from the ipsilateral (ipsi) eye. ODplasticity is clearly age dependent and maximal during the critical period (CP), in which a brief period of MD for four days induces an OD-shift of visually evoked responses in V1 towards the open eye (Hubel \& Wiesel, 1970; Gordon \& Stryker, 1996; Dräger, 1978). This juvenile OD- shift is mainly mediated by a reduction of deprived eye responses in V1 and is temporally confined to the critical period for OD-plasticity between P 20 - P 35 (Sawtell et al., 2003; Kaneko et al., 2008; for review see Espinosa and Stryker, 2012). After the CP, OD-plasticity is gradually decreasing and can only be induced after seven days of MD in standard cage (SC) raised mice until P 110 (Lehmann \& Löwel, 2008). After P 110, the classical OD-plasticity is gone in standard cage WT mice and cannot be induced even after longer deprivation times up to 14 days (Lehmann \& Löwel, 2008).

A molecular candidate that is possibly regulating the cellular basis of this critical period plasticity in mice is the postsynaptic density (PSD) protein PSD-95. It is generally found in the postsynaptic density of excitatory synapses (Karnau et al., 95; El-Husseini, 2000; Cho et al., 1992; Schenker et al., 1996; Hunt et al., 1996) and its expression increases contemporaneously with the onset of eye opening and therefore also with the beginning of visual experience (Yoshii et al., 2003). PSD-95 is required for activity-driven synapse stabilization in the brain and promotes the maturation of AMPA receptor-silent excitatory synapses in hippocampal neurons (Ehrlich et al., 2007; Stein et al., 2003; Béïque et al., 2006; Levy et al., 2015) and in the visual cortex (Huang et al., 2015).

Fagiolini et al. (2003) found that OD-plasticity in juvenile PSD-95 KO mice displays the same characteristics as it does in WT mice after 4 days of MD. Since it was unknown if adult PSD-95 KO mice would show similar OD-plasticity as WT mice and if the protein PSD-95 is important 
for the closure of the CP for OD-plasticity, adult PSD-95 KO mice were tested for OD-plasticity and further, if the protein is essential for the closure of critical periods (experiments done by Götze and Stodieck, Löwel laboratory, Göttingen).

It was tested whether the loss of PSD-95 affects the closure of the CP for OD-plasticity in young adult (< P 110) and old (> P 110) PSD-95 KO mice. Additionally, the number of silent synapses in the V1 of WT and PSD-95 KO animals was measured (Huang: doctoral thesis, 2013; Huang et al., 2015), which revealed that in PSD-95 KO mice, the number of AMPA silent synapses was similar to that of WT mice (55\%) before eye opening but remained high throughout the development and did not even decline after the CP for OD-plasticity (Figure 20).

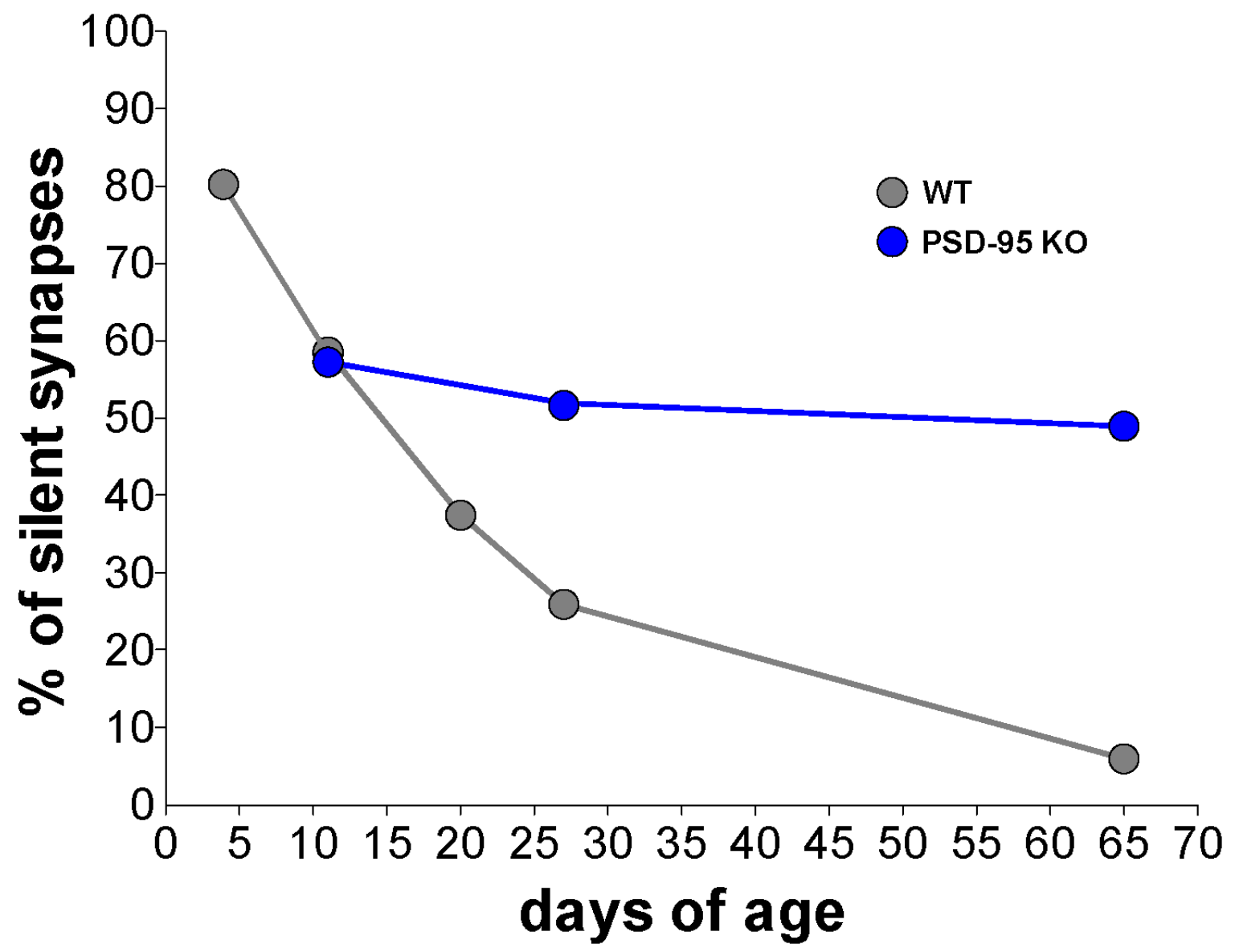

Figure 20: Number of AMPA receptor silent synapses in percent (\%) measured in the VC of WT and PSD-95 KO mice. The number of AMPA receptor silent synapses is gradually decreasing in WT mice (gray) from $80 \pm 0.05 \%$ (P $3-P 5)$ to $58.6 \pm 0.04 \%$ (P $10-P 12)$ and further to $37.5 \pm 0.06 \%$ (P $19-P 21)$. During the critical period, the numbers of AMPA silent synapses reach $26 \pm 0.05 \%$ (P $25-$ P 30) which further decrease even below $9 \%$ after the critical period. In contrast, the numbers of AMPA receptor silent synapses remain high in PSD-95 KO mice (blue) throughout life about 55 \%. Data obtained by Huang (doctoral thesis, 2013; Huang et al., 2015). 
These data indicate that PSD-95 is not only responsible for anchoring AMPARs but also absolutely required for the experience-dependent maturation of excitatory synapses onto pyramidal cells in the visual cortex after eye opening (Huang et al., 2015).

Using optical imaging of intrinsic signals in vivo (Cang et al., 2005), OD-plasticity was measured in WT and PSD-95 KO mice by comparing the response amplitudes in the binocular region of the visual cortex after visual stimulation of either the contralateral (contra) or the ipsilateral (ipsi) eye in control mice without MD and in mice after either 4 or 7 days of MD.

Experiments were performed by Bianka Götze (number of tested animals: $n=33$ ) and Sophia Stodieck (number of tested animals: $n=28$ ).

All control animals without MD showed a stronger visual cortical activation after visual stimulation of the contra eye than after visual stimulation of the ipsi eye, demonstrating the ocular dominance of the contralateral eye in the binocular region of V1 (Figure 21). The ocular dominance indices in both WT (P 97 - 488) and PSD-95 KO (P 83 - 496) mice without MD showed positive values indicating contralateral dominance (Figure 21) as well as the representative 2-dimensional color coded ocular dominance maps in the binocular part of the left V1, which showed warm, red colors (Figure 21). 
A
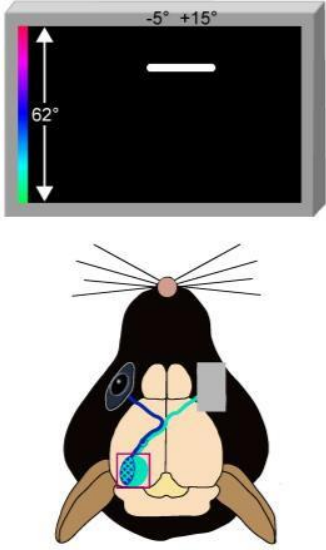

B
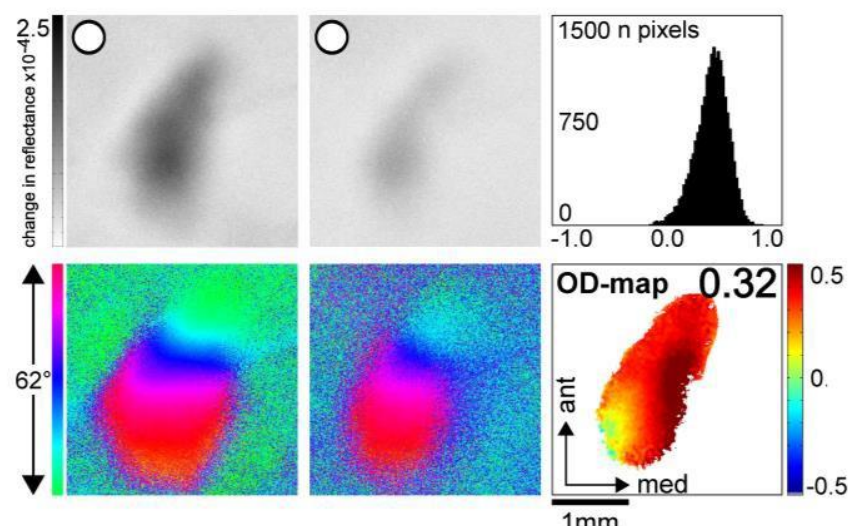

\section{histogram}

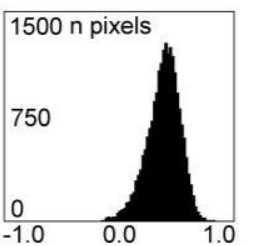

PSD-95 KO no MD

D
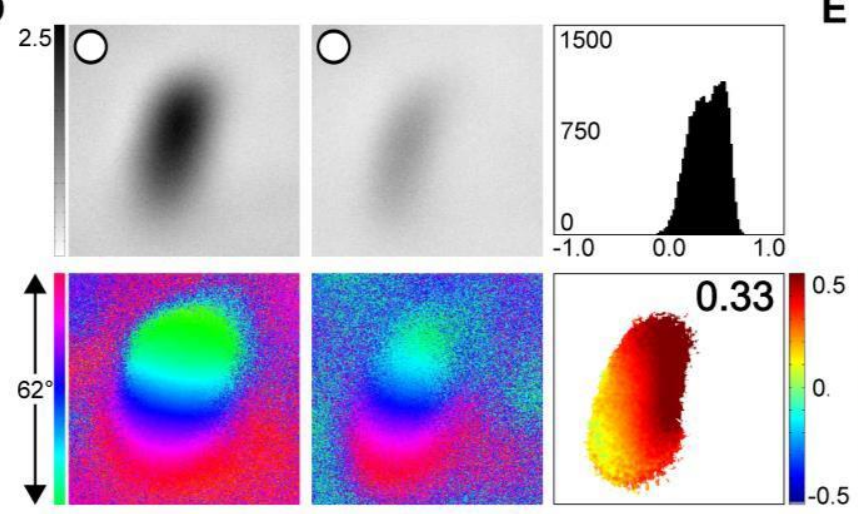

E
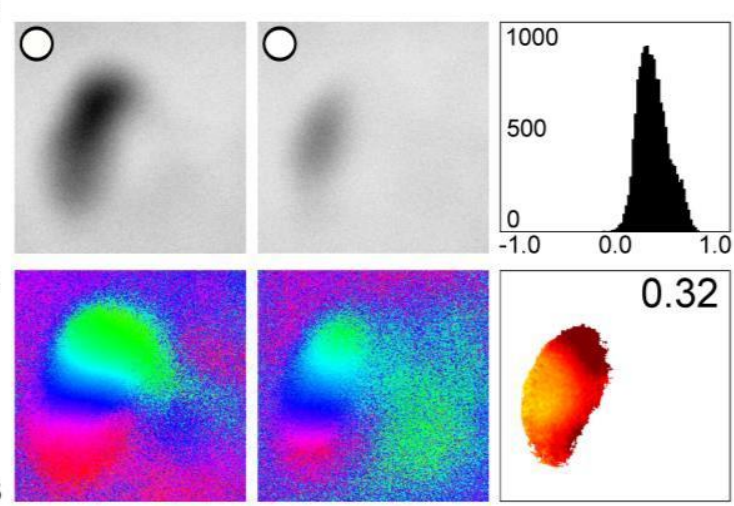

Figure 21: Representative examples of ocular dominance maps and indices in WT and PSD-95 KO mice without

MD obtained with optical imaging of intrinsic signals in vivo. (A) Optical imaging maps of contralateral (contra) and ipsilateral (ipsi) cortical responses to visual stimulation of either eye with a horizontal moving bar (elevation) of $20^{\circ}$ are displayed from (B, C) two WT mice and (D, E) two PSD-95 KO mice without MD, respectively. Grayscale coded response magnitude maps (top rows, expressed as fractional change in reflectance $\times 10^{-4}$ ), color-coded phase maps of retinotopy (bottom rows), histograms of the ocular dominance (OD)-scores (top right of panels) and color-coded OD-maps (right bottom) including the average ocular dominance index (ODI) value, are illustrated. In control mice of both genotypes, the activity patches evoked by stimulation on the contra eye were always darker than those evoked by ipsi eye stimulation. The average ODI was always positive and warm, red colors prevailed in the 2-dimensional OD-map, indicating contralateral dominance. 
WT mice after 7 days of MD showed a stronger visual cortical activation after visual stimulation of the ipsi eye than after visual stimulation of the contra eye, demonstrating OD- plasticity and ocular dominance of the ipsilateral eye in the binocular region of V1 (Figure $22 \mathrm{~A}, \mathrm{~B}$ ). In contrast to that, PSD-95 KO mice showed a change of OD which was mediated by a significant weakening of cortical responses after visual stimulation of the deprived, contralateral eye (Figure $22 \mathrm{C}, \mathrm{D})$. The ocular dominance indices in both WT (P 74 - 82) and PSD-95 KO (P 70 - 99) mice showed negative ODI values indicating ipsilateral dominance as well as the representative 2-dimensional color coded ocular dominance maps in the binocular part of the left V1, which showed cold, blue and green colors and the histogram of $(C-I / C+I)$ was shifted to the left (Figure 22$)$.

A
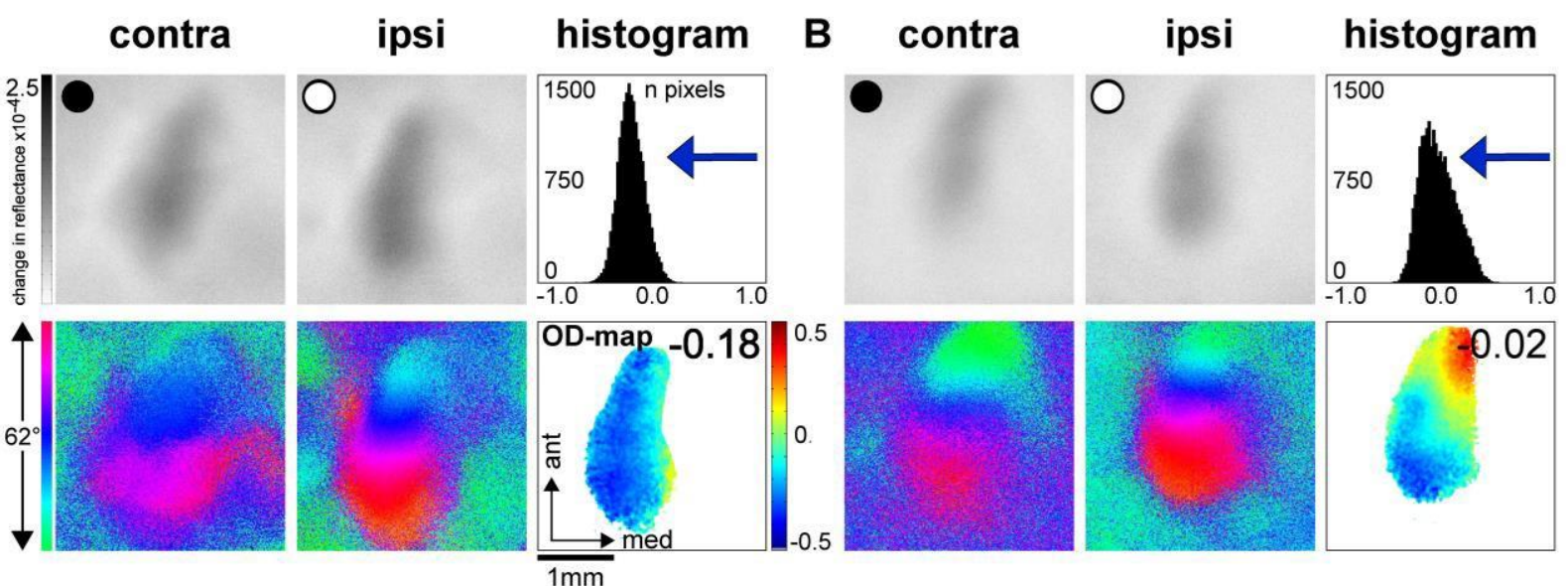

C

PSD-95 KO after 7 days of MD
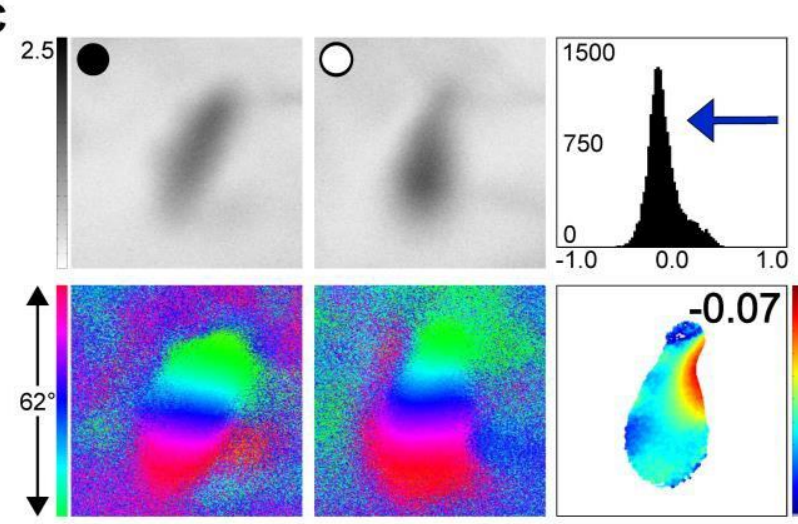

D
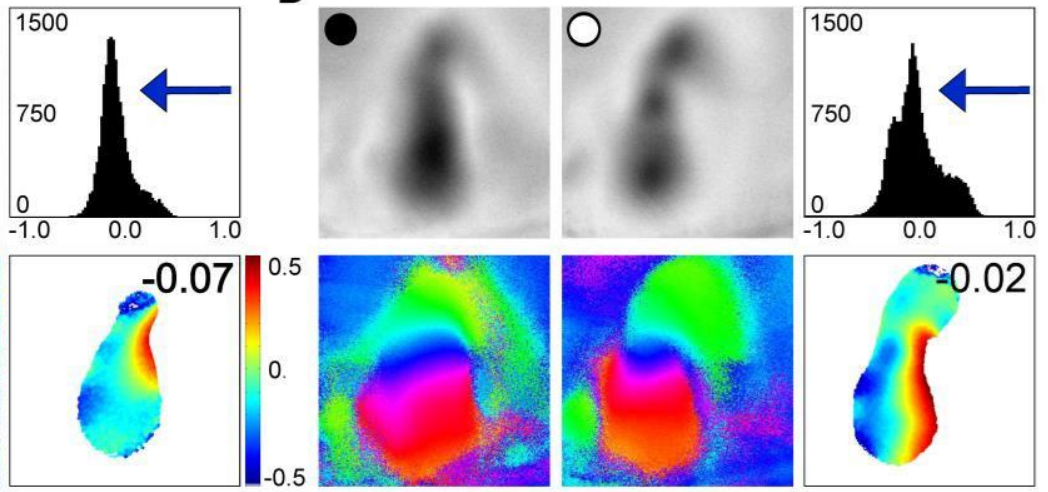

Figure 22: Representative examples of ocular dominance maps and indices in WT and PSD-95 KO mice after 7 days of MD (< P110) obtained with optical imaging of intrinsic signals in vivo. Optical imaging maps of contra- and ipsilateral cortical responses to visual stimulation of either eye with a horizontal moving bar (elevation) of $20^{\circ}$ are displayed from (A, B) two WT mice and (C, D) two PSD-95 KO mice after 7 days of MD, respectively. Grayscale coded response magnitude maps (top rows, expressed as fractional change in 
reflectance $\times 10^{-4}$ ) with black circles indicating monocular deprivation of the eye and white circles indicating that the respective eye remained open. Color-coded phase maps of retinotopy (bottom rows), histograms of the OD-scores (top right of panels) and color-coded OD-maps (right bottom including the average ODI value), are illustrated. After 7 days of MD there was an OD-shift in both genotypes. Activity patches evoked by stimulation of the contralateral (contra) eye were equally strong to that after ipsi eye stimulation, the histogram shifted to the left, the average ODI was negative and cold, blue colors prevailed in the 2dimensional OD-map. Hence, indicating ipsilateral dominance and ocular dominance plasticity.

In contrast to young adult WT mice (<P 110) which showed OD-plasticity, 7 days of MD in WT mice older than P 110 (P 123 - 311) revealed a stronger visual cortical activation after visual stimulation of the contra eye than after visual stimulation of the ipsi eye, demonstrating ocular dominance of the contralateral eye in the binocular region of V1 (Figure $23 \mathrm{~A}, \mathrm{~B}$ ). Ocular dominance indices had positive values and the 2-dimensional color coded ocular dominance maps showed warm, red colors.

In contrast, 7 days of MD in old PSD-95 KO mice (P 232 - 507) induced a change of the OD which was mediated by a significant weakening of cortical responses after visual stimulation of the deprived, contralateral eye (Figure 23 C, D). The ocular dominance indices had negative values and the representative 2-dimensional color coded ocular dominance maps in the binocular part of the left V1 showed cold, blue and green colors. The histogram of $(\mathrm{C}-\mathrm{I} / \mathrm{C}+\mathrm{I})$ was shifted to the left, indicating ipsilateral dominance and hence OD-plasticity (Figure $23 \mathrm{C}$, D). 


\section{WT after 7 days of MD}

A contra

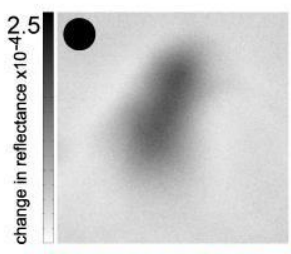

ipsi

histogram

B

contra
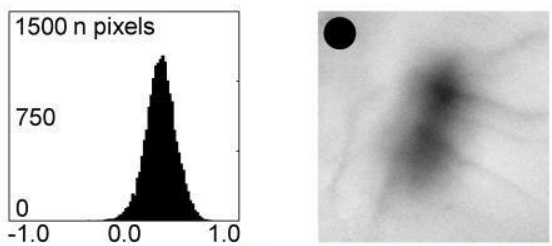

ipsi

histogram
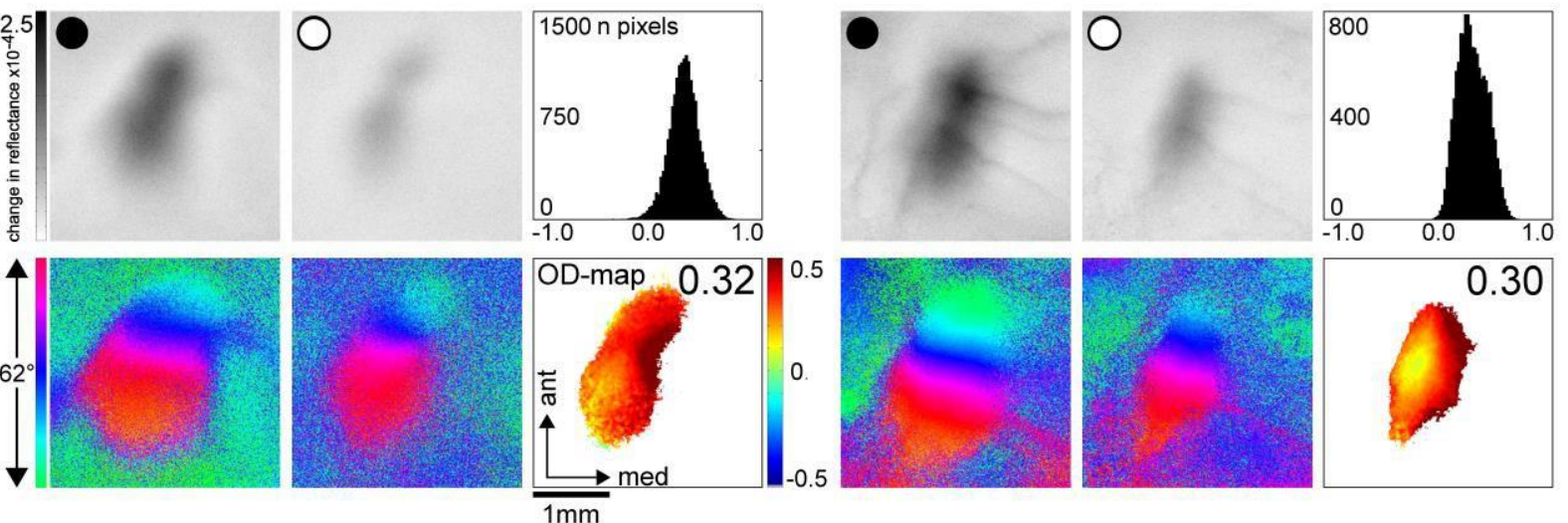

C

PSD-95 KO after 7 days of MD
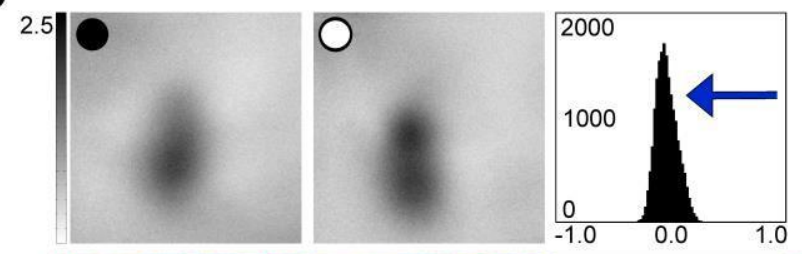

D
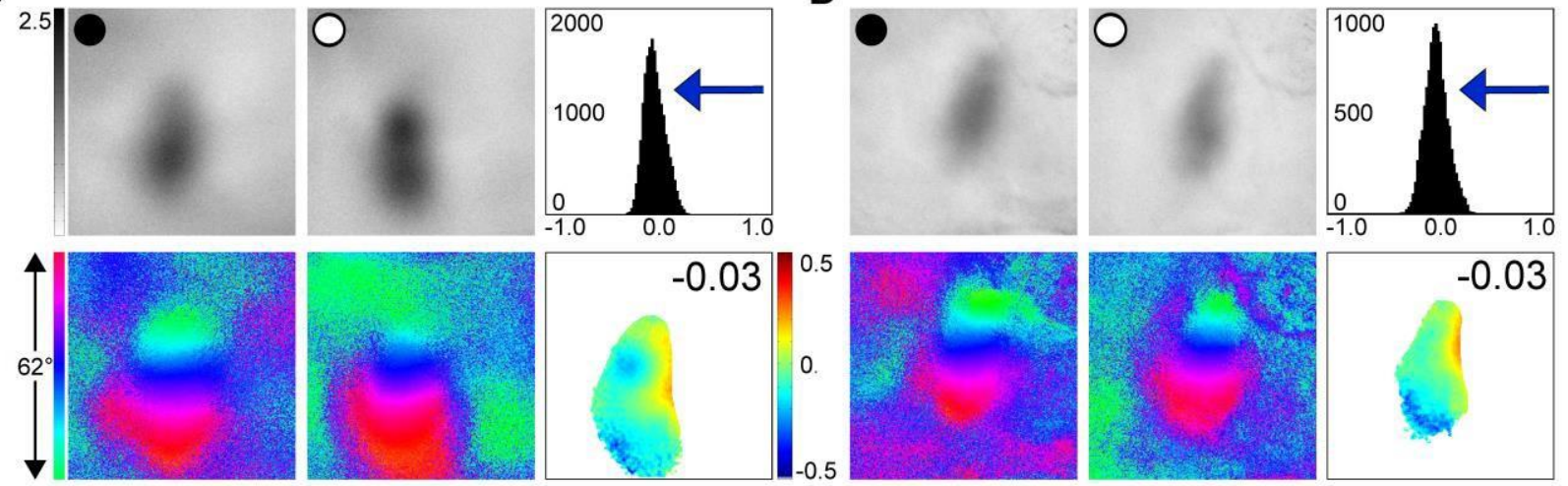

Figure 23: Representative examples of ocular dominance maps and indices in WT and PSD-95 KO mice after 7 days of MD (> P110) obtained with optical imaging of intrinsic signals in vivo. Optical imaging maps of contralateral and ipsilateral cortical responses to visual stimulation of either eye with a horizontal moving bar (elevation) of $20^{\circ}$ are displayed from (A, B) two WT mice and (C, D) two PSD-95 KO mice after 7 days of MD, respectively. Grayscale coded response magnitude maps (top rows, expressed as fractional change in reflectance $\mathrm{x}$ 10-4) with black circles indicating monocular deprivation of the eye and white circles indicating that the respective eye remained open. Color-coded phase maps of retinotopy (bottom rows), histograms of the ODscores (top right of panels) and color-coded OD-maps (right bottom including the average ODI value), are illustrated. After 7 days of MD in WT mice, the activity patches evoked by stimulation of the contra eye were always darker than those evoked after ipsi eye stimulation. The average ODI was always positive and warm, red colors prevailed in the 2-dimensional OD-map, indicating contralateral dominance. In contrast, 7 days of MD in PSD-95 KO mice induced an OD-shift towards zero. Activity patches evoked by stimulation of the contralateral eye were equally strong to that after ipsilateral eye stimulation. The histogram shifted to the left, the average ODI was negative and cold, blue colors prevailed in the 2-dimensional OD-map, indicating ipsilateral dominance and hence ocular dominance plasticity. 
Since PSD-95 KO animals display a prolonged period of cortical plasticity (Huang et al., 2015), it was investigated whether this was due to reduced cortical inhibition. Therefore, the drug diazepam was administered to investigate if increased intracortical inhibition would prevent this prolonged period for OD-plasticity. To test this, ocular dominance indices and maximum response magnitude in $\mathrm{V} 1$ were measured after seven days of MD under daily treatment with diazepam (which increases the efficiency of GABAergic inhibition).

In WT mice (< P110) OD-plasticity was completely blocked after diazepam treatment. In contrast, PSD-95 KO mice still showed a strong OD-shift after seven days of MD and diazepam treatment.

WT mice treated with diazepam revealed a stronger visual cortical activation after visual stimulation of the contra eye than after visual stimulation of the ipsi eye, demonstrating ocular dominance of the contralateral eye in the binocular region of V1 (Figure 24 A, B). Ocular dominance indices were positive and 2-dimensional color coded ocular dominance maps showed warm, red colors, indicating contralateral dominance.

In contrast to WT mice, 7 days of MD in PSD-95 KO mice treated with diazepam still induced a change of the $O D$ with equally strong cortical responses after visual stimulation of the deprived eye to that after ipsi eye stimulation (Figure 24 C, D). The ocular dominance indices in these mice showed negative values and the representative 2-dimensional color coded ocular dominance maps in the binocular part of the left V1 showed cold, blue and green colors. The histogram of $(\mathrm{C}-\mathrm{I} / \mathrm{C}+\mathrm{I})$ was shifted to the left, indicating ipsilateral dominance (Figure 24 C, D). 


\section{WT after 7 days of MD + Diazepam}

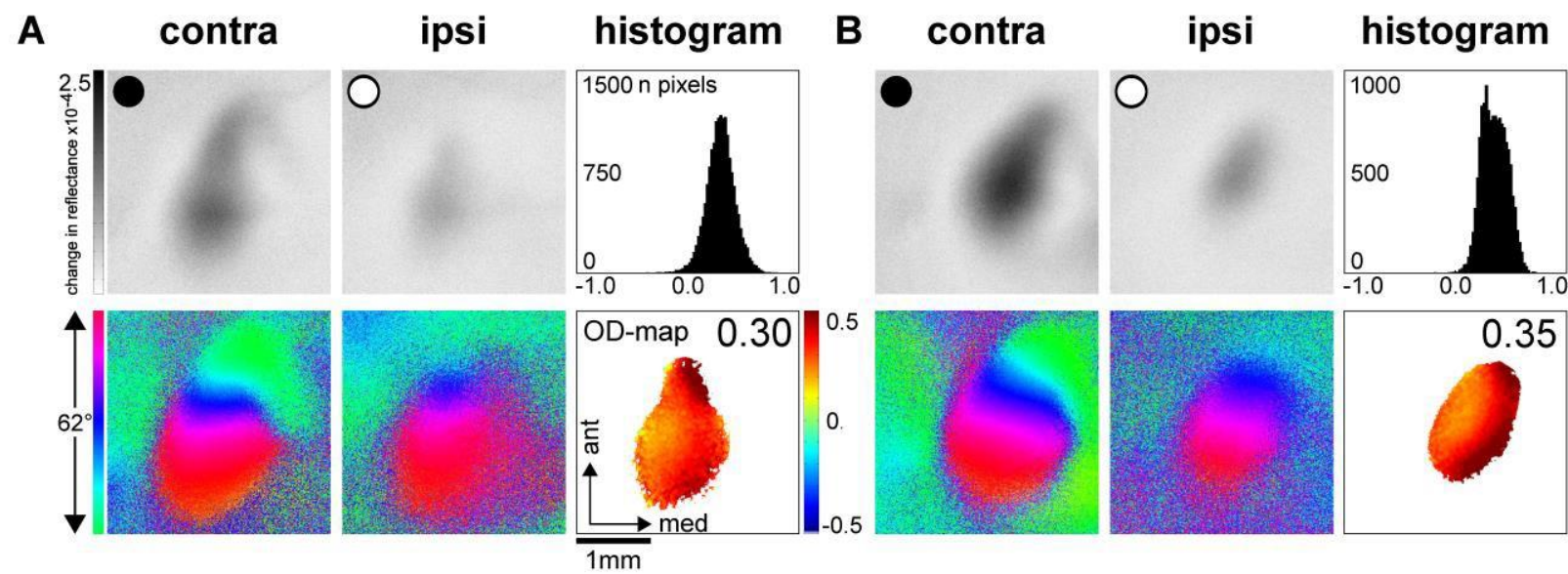

PSD-95 KO after 7 days of MD + Diazepam

C

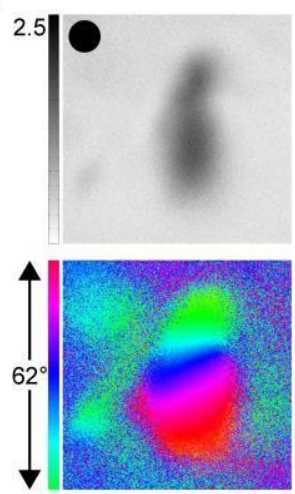

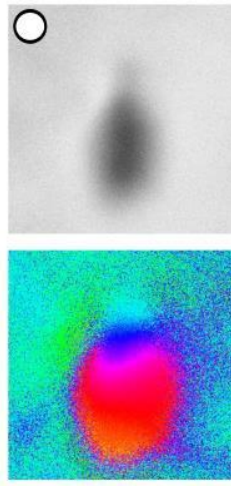

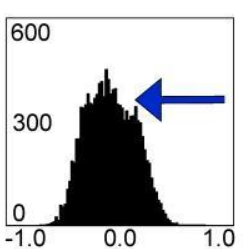

D
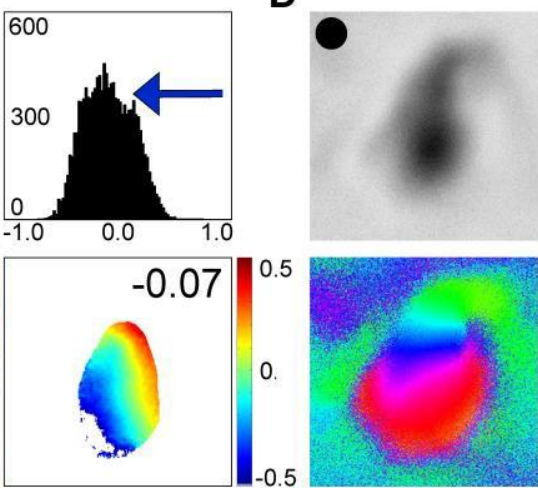
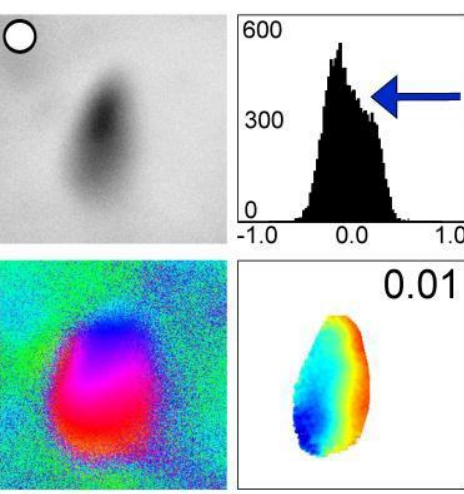

Figure 24: Representative examples of ocular dominance maps and indices in WT and PSD-95 KO mice after 7 days of MD and Diazepam treatment. Optical imaging maps of contralateral and ipsilateral cortical responses to visual stimulation of either eye with a horizontal moving bar (elevation) of $20^{\circ}$ are displayed from (A, B) two WT mice and (C, D) two PSD-95 KO mice after 7 days of MD and Diazepam treatment during the MD-period, respectively. Grayscale coded response magnitude maps (top rows, expressed as fractional change in reflectance $\mathrm{x}$ $10^{-4}$ ) with black circles indicating monocular deprivation of the eye and white circles indicating that the respective eye remained open. Color-coded phase maps of retinotopy (bottom rows), histograms of the ODscores (top right of panels) and color-coded OD-maps (right bottom including the average ODI value), are illustrated. After 7 days of MD in WT mice, the activity patches evoked by stimulation of the contra eye were always darker than those evoked after ipsi eye stimulation. The average ODI was always positive and warm, red colors prevailed in the 2-dimensional OD-map, indicating contralateral dominance. In contrast, 7 days of MD in PSD-95 KO mice induced an OD-shift towards zero even after treatment with Diazepam. The activity patches evoked by stimulation of the contra eye were equally strong to that after ipsi eye stimulation. The histogram shifted to the left, the average ODI was negative and cold, blue colors prevailed in the 2-dimensional OD-map, indicating ipsilateral dominance and hence ocular dominance plasticity. 
Quantification of the obtained data revealed that WT control animals without MD had an average ODI of $0.30 \pm 0.03(n=6)$ which was similar to that of PSD-95 KO mice $0.32 \pm 0.03$ ( $n$ = 7; Figure $26 \mathrm{~A}$ ) and statistical comparison between the two genotypes revealed no significant difference (t-test; $p=0.55)$.

In WT mice (P 74 - 82), OD-plasticity was induced after 7 days of MD (ODI < P 110 after 7d MD: $0.01 \pm 0.03 ; n=9)$, whereas it was lacking in mice after $P 110(P 123-311)$ as described before (Lehmann and Löwel, 2008) (ODI > P 110 after 7d MD: $0.30 \pm 0.02 ; n=8 ; p<0.001 ; t-$ test).

Similarly, to young adult WT mice, 7 days of MD in PSD-95 KO mice induced an OD-shift towards the open eye (ODI PSD-95 KO < P 110 after 7d MD: $0.00 \pm 0.02 ; n=8 ; p<0.001$; ttest). Surprisingly, also older animals showed OD-plasticity after 7 days of MD until at least $P$ 507 (P 232 - 507) (ODI > P 110 after 7d MD: $0.01 \pm 0.02 ; n=7 ; p<0.001$; t-test). This OD-shift was mediated by a reduction of deprived eye responses in V1 (contra no MD: $2.57 \pm 0.12$; after $7 \mathrm{~d}$ MD: $1.5 \pm 0.24 ; p<0.01$; t-test), which is a typical characteristic of juvenile ODplasticity during the CP (Sawtell et al., 2003). Ipsi eye evoked responses remained stable after MD (ipsi no MD: $1.30 \pm 0.12$; after 7 d MD: $1.37 \pm 0.22 ; p=0.81$; t-test).

In contrast to PSD-95 KO mice, the OD-shift in young adult WT-mice (< P 110) after 7 days of MD was mediated by increased visual cortical activity after open (ipsi) eye stimulation, which is a characteristic for the adult OD-plasticity (ipsi no MD: $0.96 \pm 0.13$; after $7 \mathrm{~d}$ MD: $1.6 \pm 0.19$; $p<0.05$; t-test). Cortical activation after contra eye stimulation remained similar before and after MD in WT mice (contra no MD: $1.73 \pm 0.17$; after $7 \mathrm{~d}$ MD: $1.6 \pm 0.19 ; \mathrm{p}=0.92$; t-test) (Figure $26 \mathrm{~B}$ ).

After these findings, 4 days of MD were performed in adult PSD-95 KO mice (P 186 - 480), because this short period of $M D$ is usually a hallmark of juvenile OD-plasticity during the $C P$ in WT mice raised in standard cages. Indeed, we could induce OD-plasticity in PSD-95 KO mice after only 4 days of MD (ODI: $-0.06 \pm 0.06 ; n=7)$ (Figure 25$)$, whereas in WT mice $(<P 110), 7$ days of MD were required to induce an OD-shift. Interestingly, after only 4 days of MD in adult PSD-95 KO mice, the OD-shift was also mediated by a reduction of deprived eye responses and therefore juvenile-like (contra after $4 \mathrm{~d}$ MD: $1.26 \pm 0.16 ; \mathrm{p}<0.001$; t-test) (Figure $26 \mathrm{~B}$ ). 


\section{PSD-95 KO after 4 days of MD}

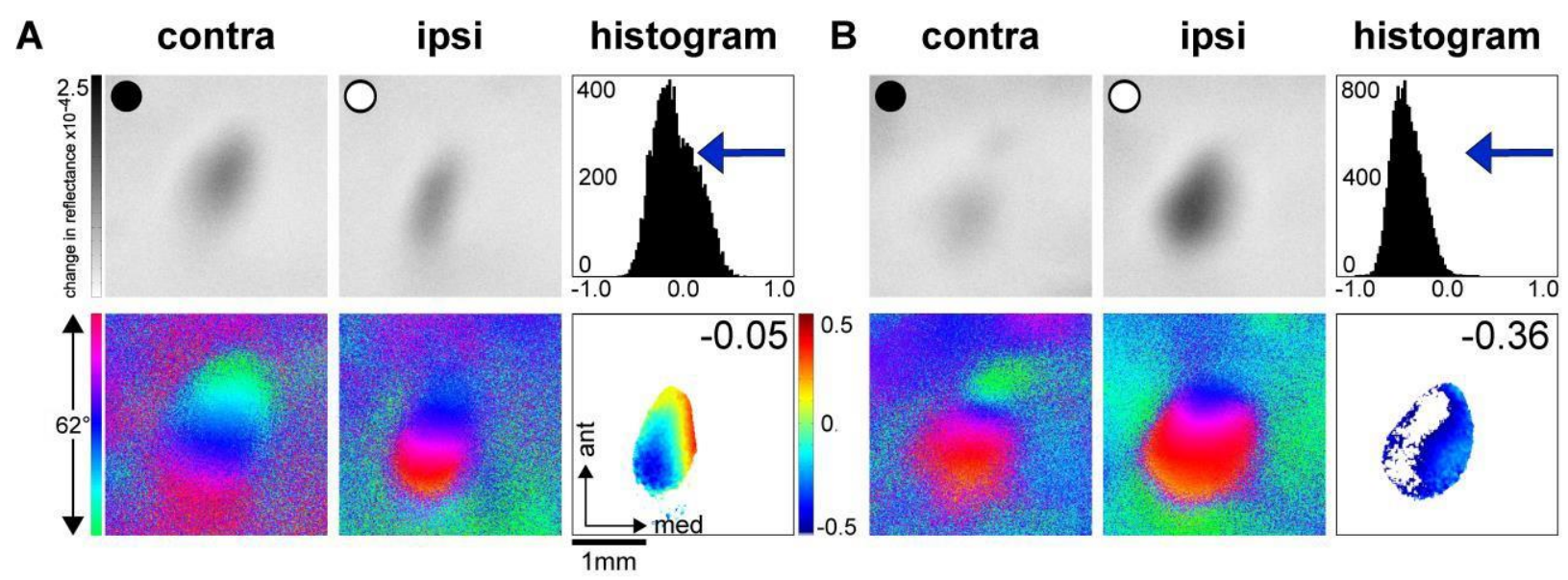

Figure 25: Representative examples of ocular dominance maps and indices in PSD-95 KO mice after 4 days of

MD. Optical imaging maps of contralateral and ipsilateral cortical responses to visual stimulation of either eye with a horizontal moving bar (elevation) of $20^{\circ}$ are displayed from two PSD-95 KO mice after 4 days of MD, respectively. Grayscale coded response magnitude maps (top rows, expressed as fractional change in reflectance $x$ $10^{-4}$ ) with black circles indicating monocular deprivation of the eye and white circles indicating that the respective eye remained open. Color-coded phase maps of retinotopy (bottom rows), histograms of the ODscores (top right of panels) and color-coded OD-maps (right bottom including the average ODI value), are illustrated. Four days of MD in PSD-95 KO mice induced an OD-shift towards zero, which is usually beyond the phase for OD-plasticity in standard cage raised WT mice (> P 110). Activity patches evoked by stimulation of the contra eye were less strong in comparison to ipsi eye stimulation. The histogram shifted to the left, the average ODI was negative and cold, blue colors prevailed in the 2-dimensional OD-map, indicating ipsilateral dominance and hence ocular dominance plasticity. 


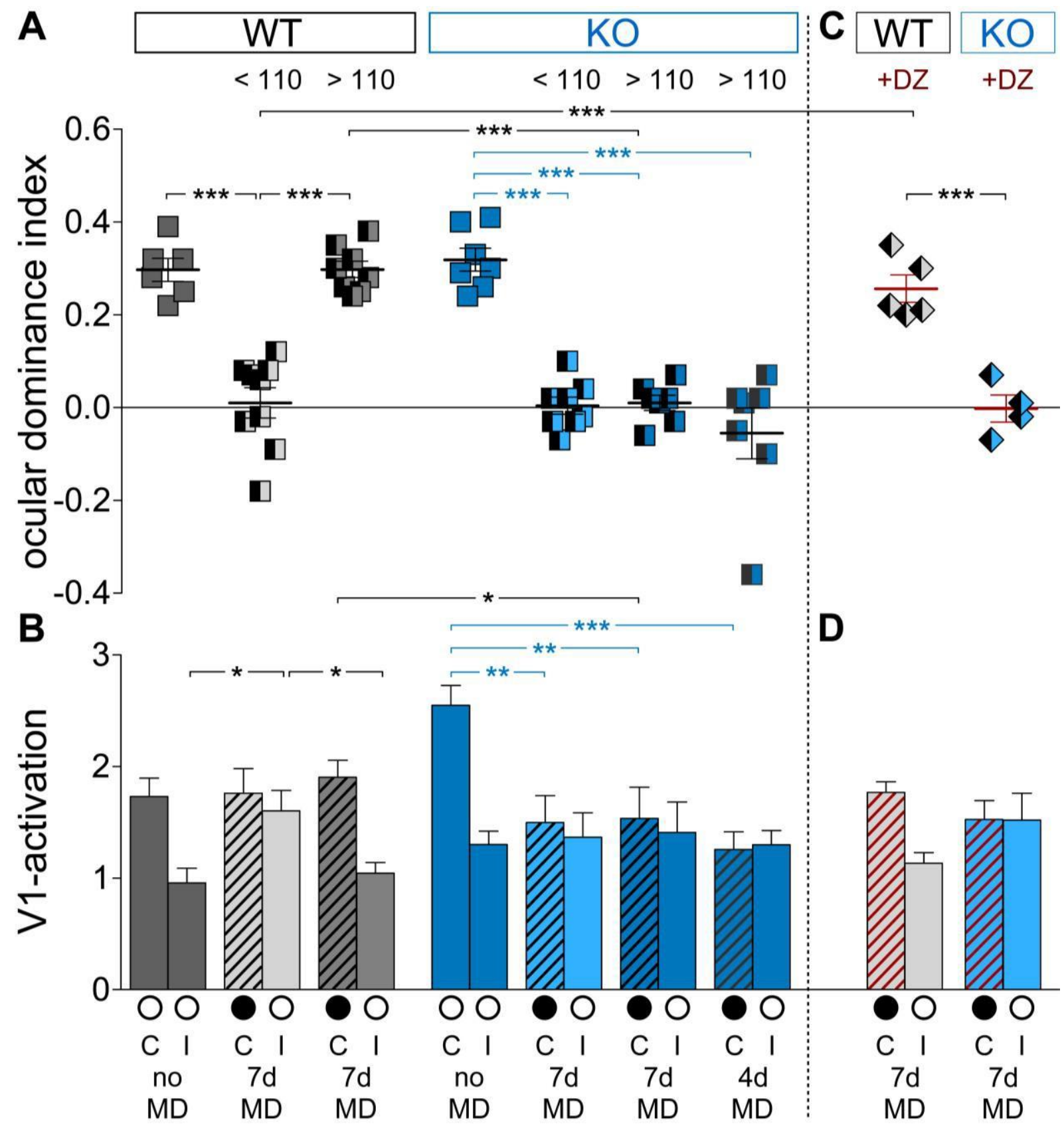

Figure 26: Ocular dominance indices and average response magnitudes of WT $(n=6)$ and PSD-95 KO mice $(n=$ 7) without MD, after seven days of $M D<P 110$ (WT, $n=9$ / KO, n = 8), > P 110 (WT, n= 8/ KO, n = 7), after 4 days of MD in PSD-95 KO ( $=7)$ and after 7 days of MD with diazepam treatment $(W T, n=5 / K O, n=4)$. (A) Positive ODI values indicate dominance of the contralateral eye, negative ODI values indicate ipsilateral dominance. Unicolored square symbols represent ODI values of individual animals without MD, half-filled square symbols represent individual animals with MD; means are marked by thick horizontal lines and the standard deviation is illustrated with small horizontal lines. WT (gray) and PSD-95 KO mice (blue) without MD showed ocular dominance of the contralateral eye and similar ODI values ( $p=0.55, t$-test). Seven days of monocular deprivation in adult WT and PSD-95 KO mice (< P 110) induced a significant OD-shift towards the open eye ( $p<$ 0.001 , t-test for both comparisons). After seven days of MD, there was no significant difference between both genotypes (t-test, $p>0.05)$. In contrast to that, seven days of monocular deprivation in old WT mice (>P 110) 
could not induce an OD-shift towards the open eye anymore ( $p=0.98$, t-test). In contrast, 7 days of MD old PSD$95 \mathrm{KO}$ mice (> P 110) still induced a significant OD-shift towards the open eye ( $<<0.001, t$-test). After seven days of $M D$, there was a significant difference between both genotypes $>P 110(p<0.001, t$-test). Only four days of MD in old PSD-95 KO mice (> P 110) were sufficient to induce a strong juvenile-like OD-shift towards zero $(P<$ 0.001 , t-test). (C) Diazepam treatment during a seven days MD-period in WT mice (<P 110) completely abolished the OD-shift, whereas it still persisted in PSD-95 KO mice (t-test, $\mathrm{p}<0.001$ ). (B) Maximum cortical responses expressed as a change in reflectance $\times 10^{-4}$ by stimulation of the contralateral (C) or ipsilateral (I) eye in WT (gray) and PSD-95 KO mice (blue) without or with seven, or four days of MD. In all control animals without MD, cortical activation after visual stimulation of the contralateral eye was significantly higher than after ipsilateral eye stimulation ( $t$-test, $\mathrm{p}<0.01)$, reflecting the dominance of the contralateral eye in mice. In adult WT mice $(<\mathrm{P}$ 110) after seven days of $M D$, response strengths of the two eyes were no longer significantly different since the non-deprived eye responses were significantly increased compared to controls ( $t$-test, $p<0.05$ ). In adult PSD-95 KO mice, seven days of induced similar response strength of the two eyes that were no longer significantly different. In contrast to WT mice, deprived-eye responses were significantly reduced compared to controls ( $t$ test, $p<0.01$ ). In old WT mice (> P 110) after seven days of MD, the response strengths of both eyes remained significantly different (t-test, $p<0.001$ ), whereas in PSD-95 KO mice after seven days of MD, the response strengths of both eyes were not significantly different. Deprived-eye responses were still significantly reduced compared to controls (t-test, $p>0.01$ ) as well as in PSD-95 KO mice after 4 days of MD (t-test, $p<0.001)$. (D) In adult WT mice (<P 110), the potentiation of the response amplitude of the open eye was absent after diazepam treatment and the response strengths of the both eyes remained significantly different ( $t$-test, $p<0.01$ ). Interestingly, even after diazepam treatment, PSD-95 KO mice exhibited OD-plasticity and an OD-shift mediated by a juvenile-like weakening of deprived eye responses in V1. Number of tested animals from Bianka Götze ( $n=$ 33) and Sophia Stodieck $(n=28)$.

\subsection{Neural circuit changes are less stable in PSD-95 KO mice}

It was shown that in the hippocampus of PSD-95 KO mice, long-term synaptic potentiation (LTP) is enhanced (Migaud et al., 1998) and spines in PSD-95 deficient neurons are less stable after LTP induction (Ehrlich et al., 2007). Since we showed prolonged OD-plasticity in adult and even old PSD-95 KO mice (Huang et al., 2015), it was tested whether cortical plasticity is less stable in PSD-95 KO mice. Therefore, seven days of MD were performed and the initially deprived eye re-opened for either two or four days. Finally, optical imaging of intrinsic signals after either two- or four days after reopening the previously deprived eye, was performed (experiments were done together with Bianka Götze (number of tested animals: $n=20$ ), Löwel laboratory, Göttingen).

After 7 days of MD in WT mice (P 77 - 84), the previously deprived eye was re-opened for two days. Ocular dominance indices were similar to that of mice after 7 days of MD. Mice still 
showed negative ODI values as well as cold colors in the representative 2-dimensional color coded ocular dominance maps in the binocular part of the left V1- The histograms of $(\mathrm{C}-\mathrm{I} / \mathrm{C}+\mathrm{I})$ were shifted to the left, indicating ipsilateral dominance (Figure $27 \mathrm{~A}, \mathrm{~B}$ ).

After 4 days of reopening the previously deprived eye in WT mice (P 88 - 97), OD-plasticity was fully recovered. The ocular dominance indices were positive, the 2-dimensional color coded ocular dominance maps showed warm, red colors and the histogram of $(\mathrm{C}-\mathrm{I} / \mathrm{C}+\mathrm{I})$ was shifted to the right indicating contralateral dominance (Figure 27 C, D).

\section{WT 2 days reopen}

A
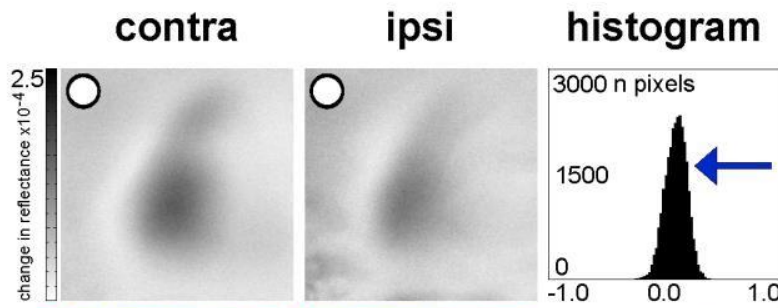

B
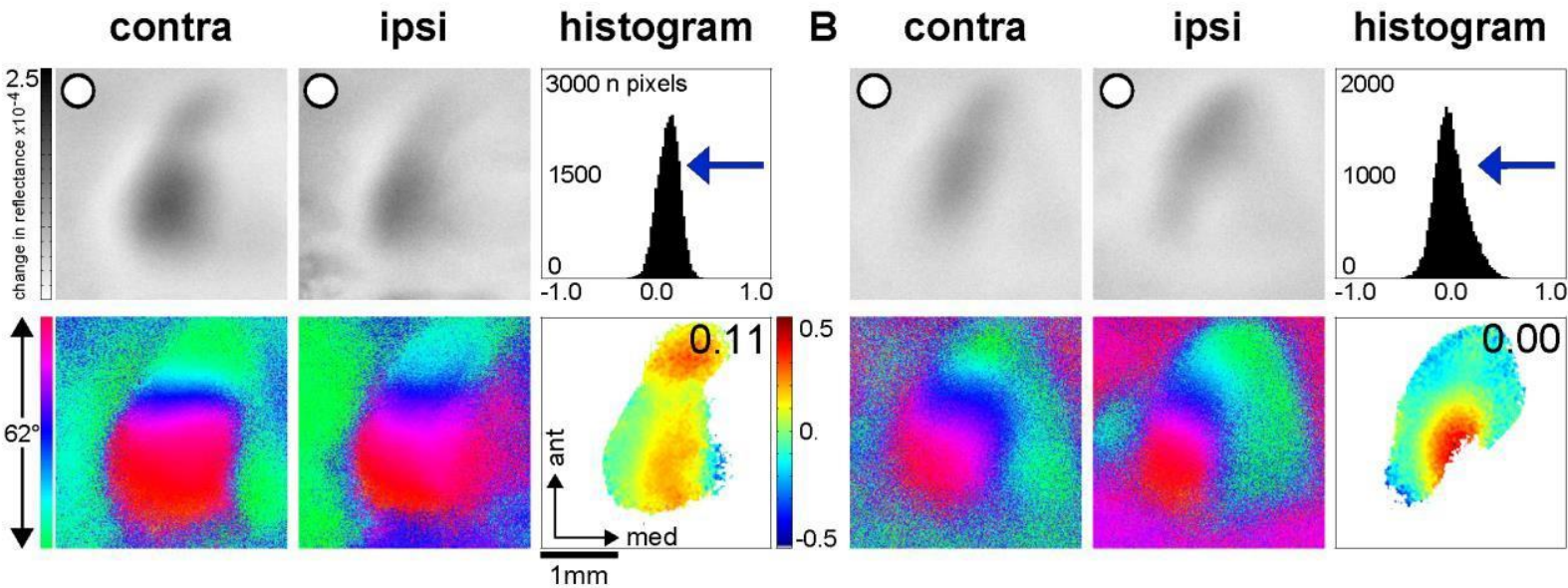

WT 4 days reopen
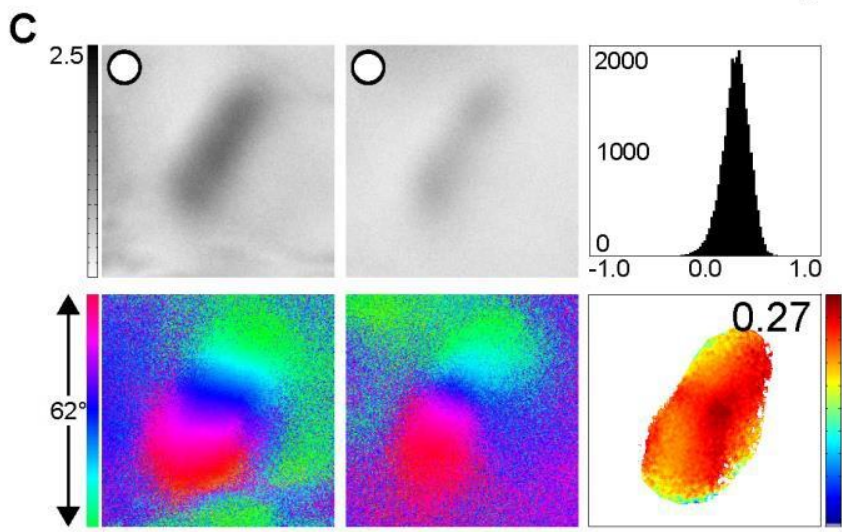

D
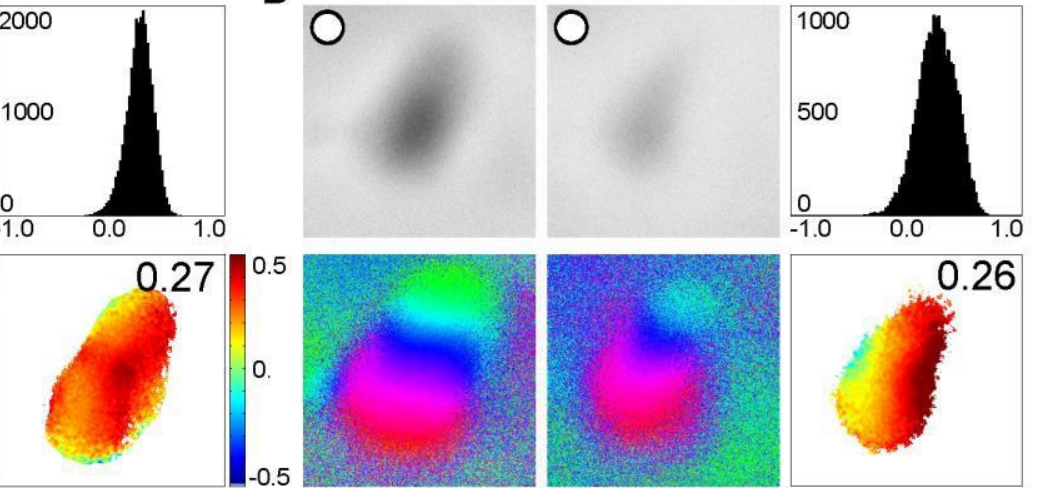

Figure 27: Representative examples of ocular dominance maps and indices in WT mice $(<\mathrm{P} 110)$ after 7 days of MD and two days or four days after reopening the previously deprived eye. Optical imaging maps of ipsiand contralateral cortical responses after visual stimulation of either eye with a horizontal moving bar (elevation) of $20^{\circ}$ are displayed from (A, B) two WT mice after 7 days of MD and two days or (C, D) 4 days after reopening the previously deprived contralateral eye, respectively. Grayscale coded response magnitude maps (top rows, expressed as fractional change in reflectance $\times 10^{-4}$ ) with white circles indicating that the respective eye remained open. Color-coded phase maps of retinotopy (bottom rows), histograms of the OD-scores (top right of panels) and color-coded OD-maps (right bottom including the average ODI value), are illustrated. After 7 days of MD and two days of reopening the contra eye, there was still an OD-shift towards zero. Activity patches 
evoked by stimulation of the contralateral (contra) eye were equally strong to that after ipsi eye stimulation, the ODI histogram shifted to the left, the average ODI was negative, and cold, blue colors prevailed in the 2dimensional OD-map, indicating ipsilateral dominance and hence ocular dominance plasticity. In contrast to that, after 4 days of reopening the previously deprived contra eye, the OD-shift was fully recovered with stronger visual cortical activation after contra eye stimulation than after ipsi eye stimulation. Positive ODI values and warm, red colors prevailed in the 2-dimensional OD-maps, indicating contralateral dominance.

In contrast to WT mice, already two days of reopening the previously deprived contralateral eye in PSD-95 KO mice (P 76 - 83), fully recovered the previous OD-shift after MD. The ocular dominance indices were positive, the 2-dimensional color coded ocular dominance maps showed warm, red colors and the histogram of $(C-I / C+I)$ was shifted to the right, indicating contralateral dominance (Figure 28).

A
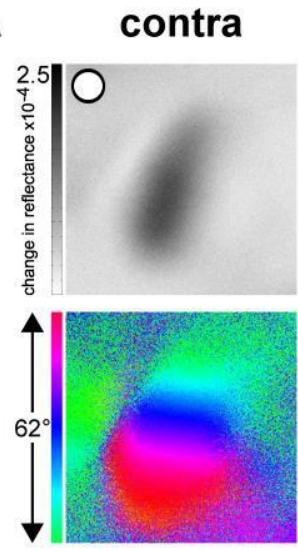

PSD-95 KO 2 days reopen

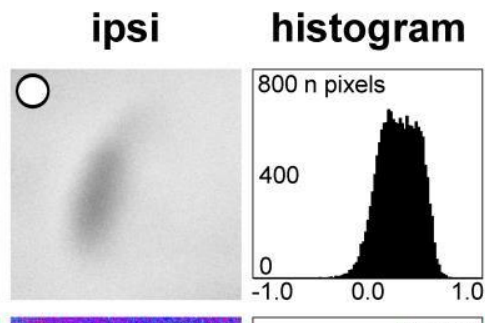

B
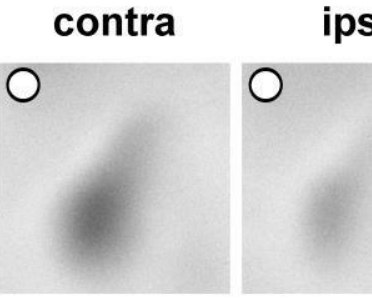

ipsi

histogram

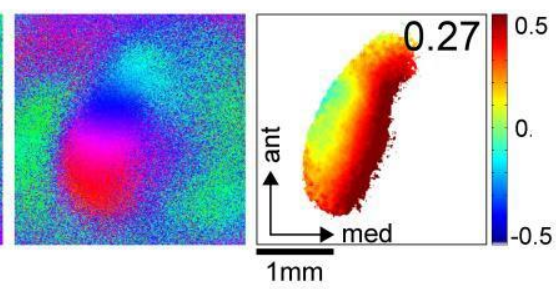

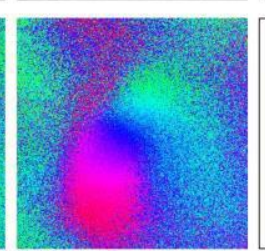
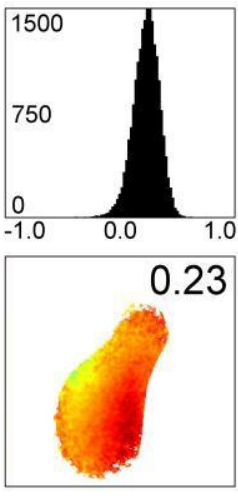

Figure 28: Representative examples of ocular dominance maps and indices of PSD-95 KO mice after 7 days of MD and reopening the previously deprived eye for two days. (A, B) Optical imaging maps of ipsi- and contralateral cortical responses to visual stimulation of either eye with a horizontal moving bar (elevation) of $20^{\circ}$ are displayed from two PSD-95 KO mice after 7 days of MD and reopening of the contra eye for two days. Grayscale coded response magnitude maps (top rows, expressed as fractional change in reflectance $\times 10^{-4}$ ), color-coded phase maps of retinotopy (bottom rows), histograms of the OD-scores (top right of panels) and color-coded OD-maps (right bottom including the average ODI value), are illustrated. After two days of reopening the previously deprived contra eye, the OD-shift of PSD-95 KO mice was fully recovered with stronger visual cortical activation after contra eye stimulation than after ipsi eye stimulation. Positive ODI values and warm, red colors prevailed in the 2-dimensional OD-maps, indicating contralateral dominance. 
Quantification of the data revealed that in PSD-95 KO mice, only 2 days of reopening the previously deprived eye were sufficient to recover the OD-shift which was induced after 7 days of MD (ODI after 7d MD: $0.00 \pm 0.02 ; n=8$; after $2 d$ reopen: $0.27 \pm 0.01 ; n=5 ; p<0.001$; t-test), whereas OD-plasticity persisted in WT mice after 2 days of reopening the previously deprived eye (ODI after $7 \mathrm{~d}$ MD: $0.01 \pm 0.03 ; n=9$; after $2 \mathrm{~d}$ reopen: $0.09 \pm 0.03 ; n=4 ; p=$ 0.2 ; t-test). It was only recovered after 4 days of reopening the previously deprived eye (ODI: $0.27 \pm 0.01 ; n=3 ; p<0.01 ;$ t-test) (Figure 29).
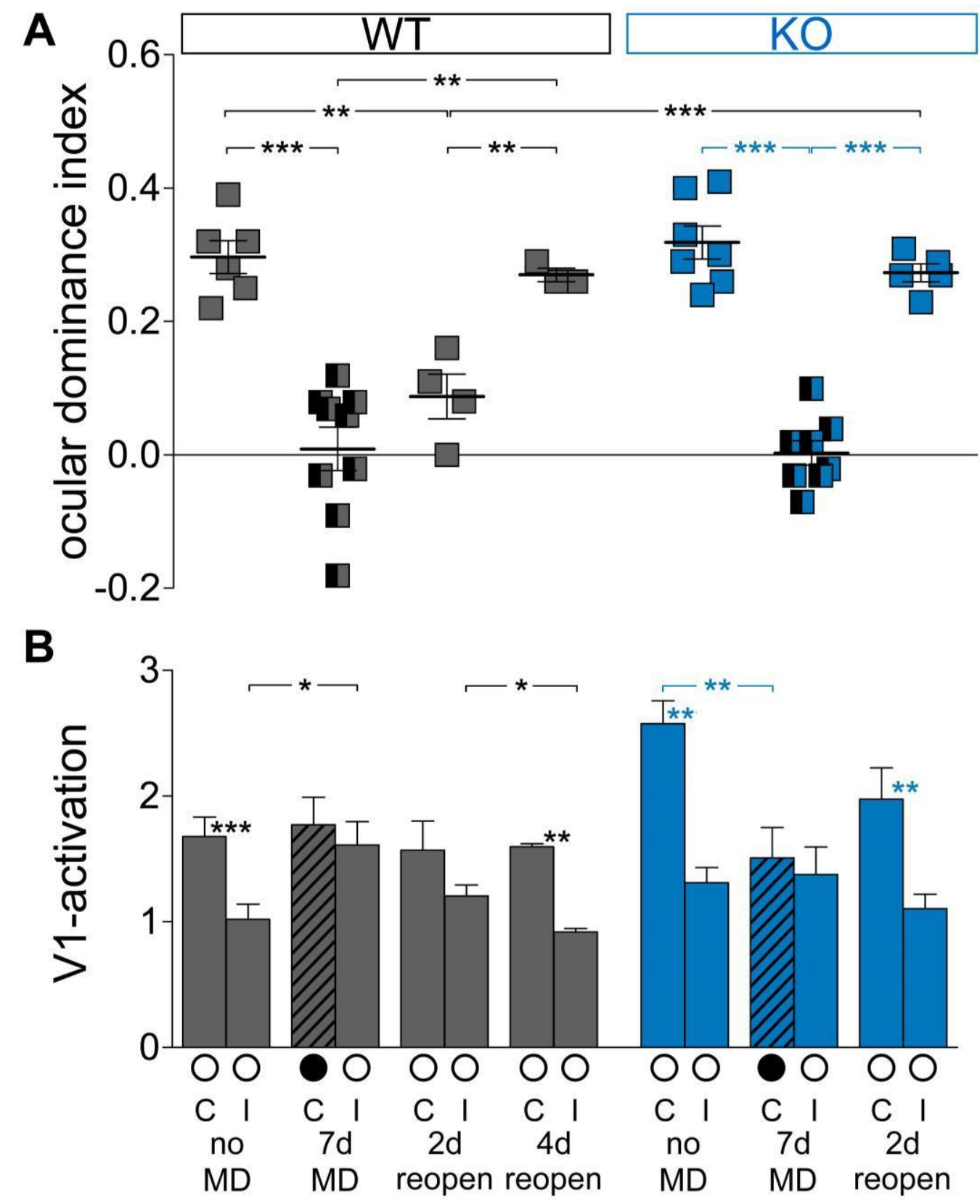

Figure 29: Ocular dominance indices and average response magnitudes of $\mathrm{WT}(\mathrm{n}=6)$ and PSD-95 KO mice $(\mathrm{n}=$ 7) without MD, after seven days of $M D<P 110(W T, n=9$ / KO, $n=8)$, after reopening the previously deprived eye for two days (WT, $n=4$ / KO, $n=5$ ), and after four days of reopening the contralateral eye in WT mice ( 
= 3). (A) Positive ODI values indicate dominance of the contralateral eye, negative ODI values indicate ipsilateral eye dominance. Unicolored square symbols represent ODI values of individual animals without MD, or a reopened contralateral eye. Half-filled square symbols represent individual animals with MD; means are marked by thick horizontal lines and the standard deviation is illustrated with small horizontal lines. WT (gray) and PSD$95 \mathrm{KO}$ mice (blue) without MD showed ocular dominance of the contralateral eye and similar ODI values ( $\mathrm{t}$-test, $\mathrm{p}$ $=0.55)$. Seven days of monocular deprivation in adult WT and PSD-95 KO mice ( $\angle P 110)$ induced a significant ODshift towards the open eye (t-test, $p<0.001$ for both comparisons). After seven days of MD, there was no significant difference between both genotypes ( $t$-test, $p>0.05$ ). Reopening the previously deprived eye for two days in WT mice revealed no change and animals still exhibited an OD-shift towards zero. In contrast, the ODshift after MD was completely recovered in PSD-95 KO mice after two days ( $t$-test, $p<0.001$ ). After 4 days of reopening the formerly deprived eye, the OD-shift was also recovered in WT mice (t-test, $p<0.01)$. (B) Maximum cortical response amplitudes in the visual cortex (expressed as a change in reflectance $\times 10^{-4}$ ) after visual stimulation of the contralateral (C) or ipsilateral (I) eye in WT (gray) and PSD-95 KO mice (blue), respectively. In all control animals without MD, cortical activation after visual stimulation of the contralateral eye was significantly higher than after ipsilateral eye stimulation (t-test, $p<0.01$ ), reflecting dominance of the contralateral eye in mice. In adult WT mice (< P 110) after seven days of MD, response strengths of the two eyes were no longer significantly different. The non-deprived eye responses were significantly increased compared to controls (t-test, $p<0.05$ ). In contrast to WT mice, seven days of MD in adult PSD-95 KO mice induced response strength of the two eyes that were equally strong. Deprived-eye responses were significantly reduced compared to controls (t-test, $p<0.01$ ). After two days of reopening the contralateral eye after MD in WT mice, visual cortical responses of both eyes were still equally strong (t-test, $p>0.05$ ). In contrast, the reduction of contra eye responses in PSD-95 KO mice after MD was reversed with stronger cortical activation after contra eye stimulation in comparison to that after ipsi eye stimulation (t-test, $p<0.01$ ). In WT mice, the previously deprived eye had to be re-opened for four days to recover from the OD-shift after MD with stronger cortical activation after contra eye stimulation in comparison to that after ipsi eye stimulation ( $p<0.01$, t-test). Number of tested animals from Bianka Götze $(n=20)$ and Sophia Stodieck $(n=22)$.

These data reveal that changes in the visual cortex of adult PSD-95 KO mice are more transient and reverse faster than in WT mice, indicating that functional properties of neurons may not be consolidated and stabilized in networks without PSD-95. 


\subsection{V1-specific knockdown of PSD-95 expression before maturation (P 0 ) restores juvenile-like OD-plasticity}

Since a global knockout of PSD-95 can preserve a juvenile form of OD-plasticity lifelong, it was tested whether a knockdown (KD) of PSD-95 only in the visual cortex is already sufficient to prevent the closure of the CP for OD-plasticity and whether it can restore juvenile-like ODplasticity in adult WT mice. To this end, PSD-95 expression was silenced in WT mice (P $0-P$

2) selectively only in the visual cortex of both hemispheres with an adeno-associated virus (AAV) expressing a short hairpin (sh) RNA targeting PSD-95 (AAV - sh95), or an AAV expressing short hairpin RNA luciferase tagged with GFP for controls (AAV - shlc) (most virus injections were done by Huang; Schlüter laboratory, ENI, Göttingen). I tested OD-plasticity after 4 days of $M D$ at $~ P ~ 80$ with optical imaging of intrinsic signals in vivo.

Control injected (AAV - shlc) animals after 4 days of MD showed a stronger visual cortical activation after visual stimulation of the contra eye than after visual stimulation of the ipsi eye, demonstrating unchanged ocular dominance of the contralateral eye in the binocular region of $\mathrm{V} 1$ after $\mathrm{MD}$ (Figure $30 \mathrm{~A}, \mathrm{~B}$ ). Ocular dominance indices were positive, and the representative 2-dimensional color coded ocular dominance maps in the binocular part of the left V1 showed warm, red colors, indicating contralateral dominance

In contrast to that, WT mice with a visual cortex restricted knockdown of PSD-95 in both hemispheres showed a weakening of visual cortical activation after visual stimulation of the contra eye after 4 days of MD. Hence, demonstrating OD-plasticity and ocular dominance of the ipsilateral eye in the binocular region of V1 (Figure $30 \mathrm{C}, \mathrm{D}$ ). Ocular dominance indices were negative and the 2-dimensional color coded ocular dominance maps showed cold, blue and green colors and the histogram of $(C-1 / C+1)$ was shifted to the left (Figure $30 \mathrm{C}, \mathrm{D})$. 


\section{WT shcl control after 4 days of MD}

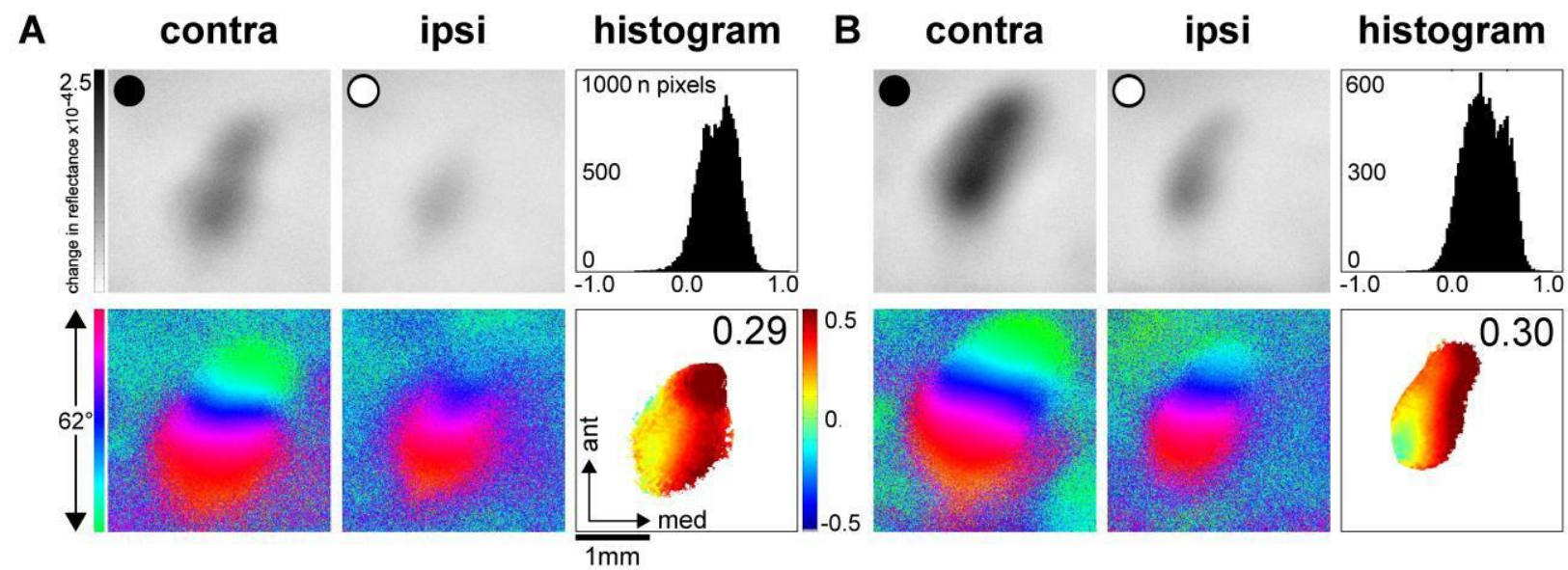

\section{WT sh95 knockdown after 4 days of MD}

C
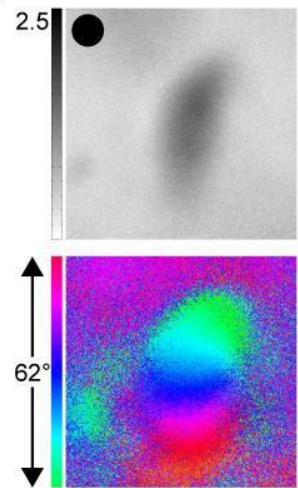
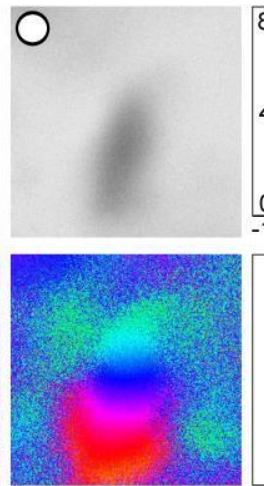
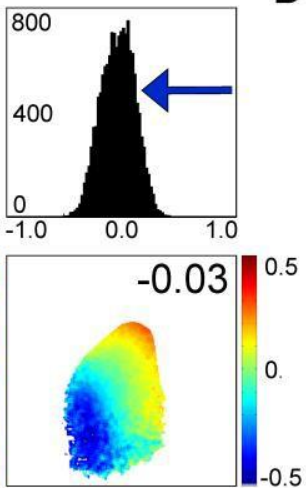

D

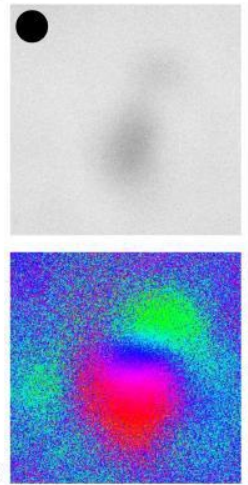

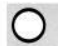

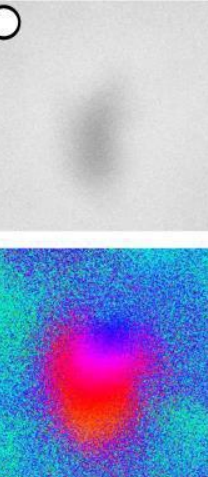

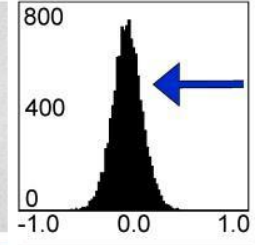

$-0.05$

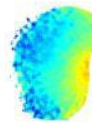

Figure 30: Representative examples of ocular dominance maps and indices in WT mice with injections of AAV shlc (control) or AAV - sh95 (PSD-95 knockdown) in V1 at P 0 - P 2 after 4 days of MD obtained with optical imaging of intrinsic signals in vivo. Optical imaging maps of contralateral and ipsilateral cortical responses to visual stimulation of either eye with a horizontal moving bar (elevation) of $20^{\circ}$ are displayed from (A, B) two WT control mice (AAV - shlc) and (C, D) two WT mice with a visual cortex restricted knockdown of PSD-95 after 4 days of MD, respectively. Grayscale coded response magnitude maps (top rows, expressed as fractional change in reflectance $\times 10^{-4}$ ) with black circles indicating monocular deprivation of the eye and white circles indicating that the respective eye remained open. Color-coded phase maps of retinotopy (bottom rows), histograms of the OD-scores (top right of panels) and color-coded OD-maps (right bottom including the average ODI value), are illustrated. After 4 days of MD, control mice showed activity patches which were stronger after visual stimulation of the contra eye than after stimulation of the ipsi eye. ODI values were positive and warm, red colors prevailed in the according color coded 2-dimensional OD-map, indicating contralateral dominance. In contrast to that, mice with a knockdown of PSD-95 exhibited an OD-shift towards zero. The activity patches evoked by stimulation of the contra eye were equally strong to that after ipsi eye stimulation. The histogram shifted to the left and the average ODI was negative. Cold, blue colors prevailed in the 2-dimensional OD-map, indicating ipsilateral dominance and hence ocular dominance plasticity. 
Quantification of the data revealed that WT mice with V1-specific PSD-95 knockdown in both hemispheres showed OD-plasticity after only 4 days of MD (ODI: $0.01 \pm 0.02 ; n=7$ ), whereas age-matched controls (AAV - shlc) did not show OD-plasticity (ODI: $0.28 \pm 0.02 ; n=4 ; p<$ 0.001 ; t-test). Interestingly, the OD-shift of PSD-95 knockdown mice was as strong as in mice with a global PSD-95 KO ( $p=0.3$, t-test) and also mediated by a reduction of deprived eye responses in V1, indicating juvenile-like OD-plasticity (shlc: contra after $4 \mathrm{~d}$ MD: $1.61 \pm 0.27$; $n=4$; sh95: contra after 4 d MD: $0.99 \pm 0.13 ; n=7$; t-test: $p<0.05)$. Cortical activation after ipsi eye stimulation remained unchanged (shlc: ipsi after 4 d MD: $0.98 \pm 0.18 ; n=4$; sh95: ipsi after 4 d MD: $0.99 \pm 0.15 ; n=7$; t-test: $p=0.97$ ) (Figure 32).

Recently, it was shown that by inactivating one hemisphere in mouse V1 (P 84 - 101), activity evoked by the ipsilateral eye was significantly reduced in the binocular part of V1 of the opposing hemisphere (Dehmel \& Löwel, 2014). These data suggest that cortico -cortical interactions play a major role for determining the ocular dominance in adult mouse V1 (Dehmel \& Löwel, 2014).

To test whether both hemispheres contributed to the OD-shift in animals with a knockdown of PSD-95 in the visual cortex after 4 days of MD, PSD-95 expression was silenced with AAV sh95 either contralateral or ipsilateral to the deprived eye in WT mice (P 0 - P 2). Optical imaging of intrinsic signals was performed after 4 days of $M D$ at $\sim P 80$.

Silencing PSD-95 expression in the contralateral hemisphere to the deprived eye (left hemisphere and location of the recording site) in WT mice revealed a weakening of visual cortical activation after visual stimulation of the contra eye after 4 days of MD. Hence, demonstrating OD-plasticity and ocular dominance of the ipsilateral eye in the binocular region of V1 (Figure $31 \mathrm{~A}, \mathrm{~B}$ ). The ocular dominance indices were negative and the 2dimensional color coded ocular dominance maps showed cold, blue and green colors, and the histogram of $(C-I / C+I)$ was shifted to the left (Figure $31 \mathrm{~A}, \mathrm{~B}$ ).

Contrary to these findings, a visual cortex restricted knockdown of PSD-95 only in the right, ipsilateral hemisphere to the deprived eye, did not change visual cortical activation after 4 days of MD. Cortical activation after visual stimulation of the contra eye was still stronger than after visual stimulation of the ipsi eye. The 2-dimensional color coded ocular dominance maps showed warm, red colors and the histogram of $(C-I / C+I)$ and the ocular dominances indices remained positive, indicating contralateral dominance (Figure 31 C, D). 


\section{WT sh95 knockdown left hemisphere after 4 days of MD}

A

\begin{abstract}
contra
\end{abstract}
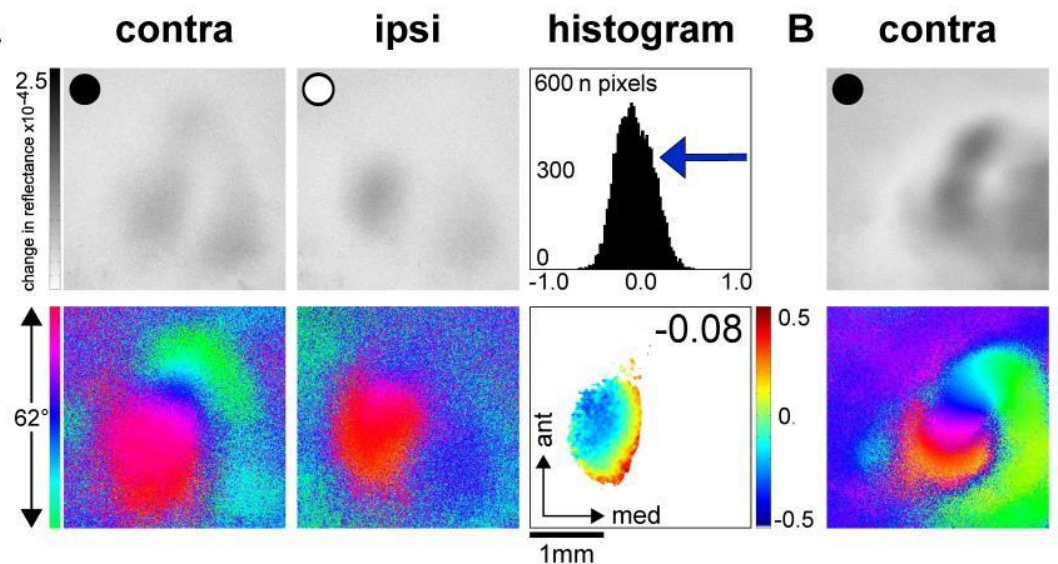

ipsi
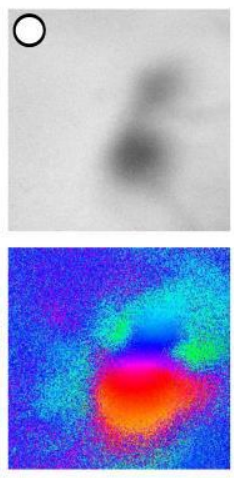

histogram
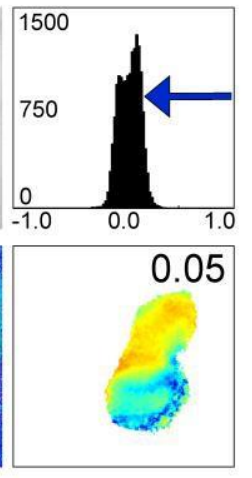

WT sh95 knockdown right hemisphere after 4 days of MD

C
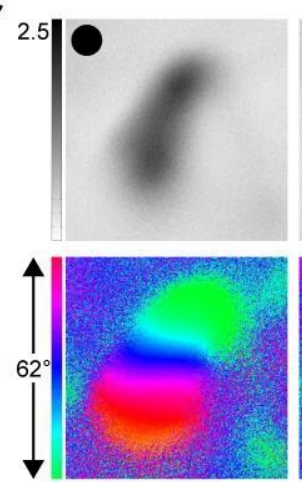
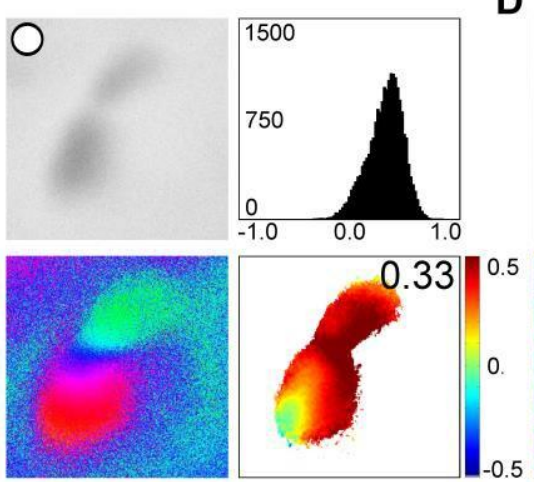

D

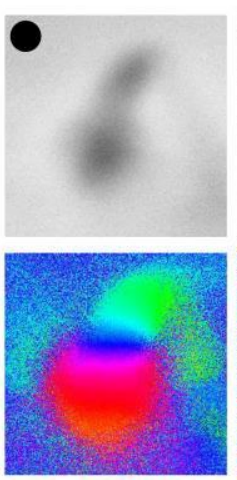

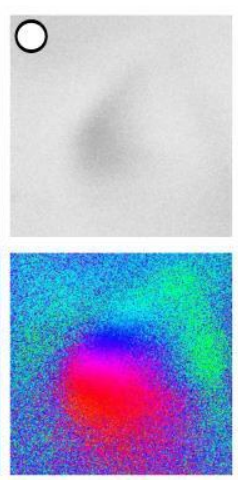
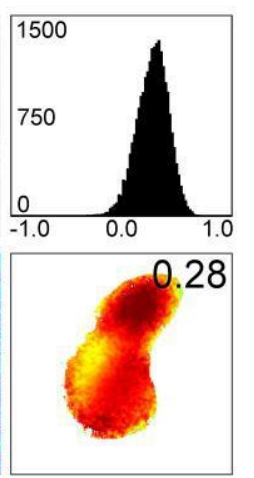

Figure 31: Representative examples of ocular dominance maps and indices in WT mice with a visual cortex restricted knockdown of PSD-95 either in the visual cortex of the left or the right hemisphere at P 0 - P 2 after 4 days of MD obtained with optical imaging of intrinsic signals in vivo. Optical imaging maps of contralateral and ipsilateral cortical responses to visual stimulation of either eye with a horizontal moving bar (elevation) of $20^{\circ}$ are displayed from $(\mathbf{A}, \mathbf{B})$ two WT mice with a knockdown of PSD-95 in the visual cortex of the left hemisphere (contralateral to the deprived eye) or (C, D) in the right hemisphere (ipsilateral to the deprived eye) after 4 days of $M D$, respectively. Grayscale coded response magnitude maps (top rows, expressed as fractional change in reflectance $\times 10^{-4}$ ) with black circles indicating monocular deprivation of the eye and white circles indicating that the respective eye remained open. Color-coded phase maps of retinotopy (bottom rows), histograms of the ODscores (top right of panels) and color-coded OD-maps (right bottom including the average ODI value), are illustrated. After 4 days of MD, mice with a knockdown of PSD-95 in the left visual cortex showed an OD-shift towards zero. Activity patches evoked by stimulation of the contralateral (contra) eye were equally strong to that after ipsi eye stimulation, the ODI histogram shifted to the left, the average ODI was negative and cold, blue colors prevailed in the 2-dimensional OD-map. Hence, indicating ipsilateral dominance and ocular dominance plasticity. In contrast to that, WT mice with a knockdown of PSD-95 in the right visual cortex showed activity patches which were stronger after visual stimulation of the contra eye than after stimulation of the ipsi eye. Positive ODI values and warm, red colors prevailed in the according color coded 2-dimensional OD-map, indicating contralateral dominance. 
Recording the contralateral visual cortex to the deprived eye in mice with a PSD-95 knockdown in the visual cortex contralateral to the deprived eye revealed a strong OD-shift towards zero (ODI: $0.05 \pm 0.03 ; n=7$ ). This OD-shift was absent in mice with a knockdown ipsilateral to the deprived eye (ODI: $0.24 \pm 0.01 ; n=5 ; p<0.001 ; t$-test) (Figure 32).

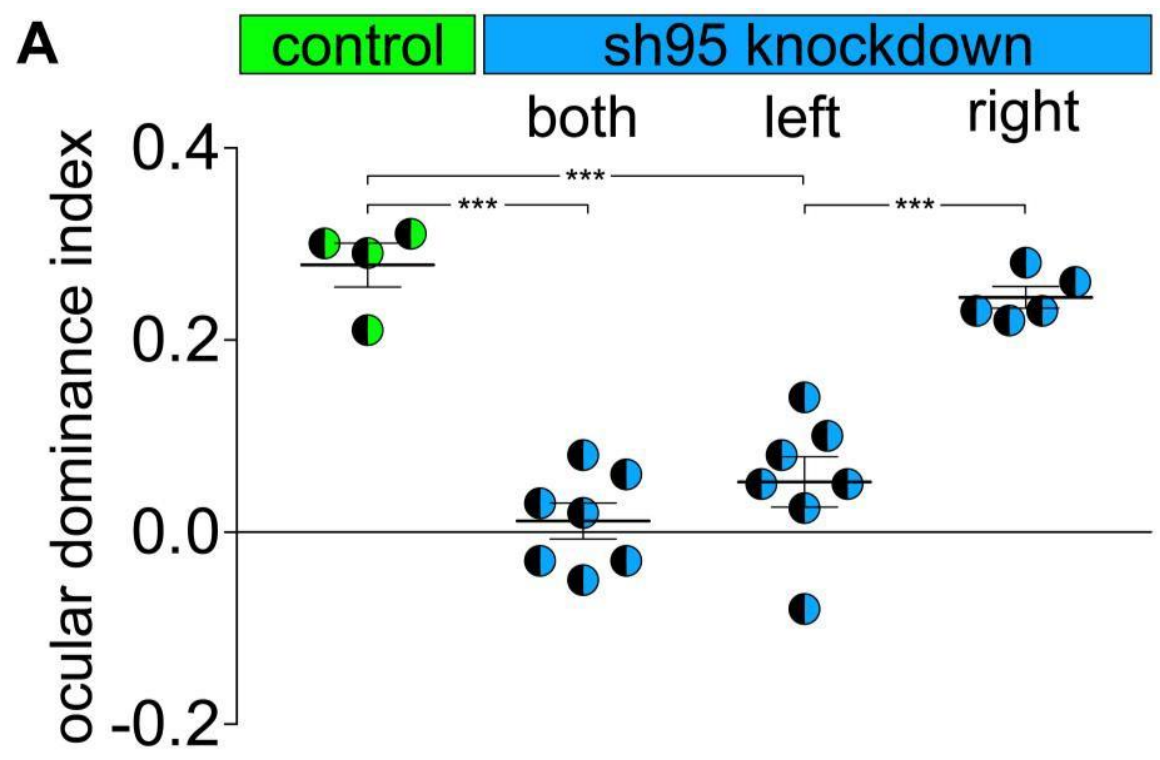

B

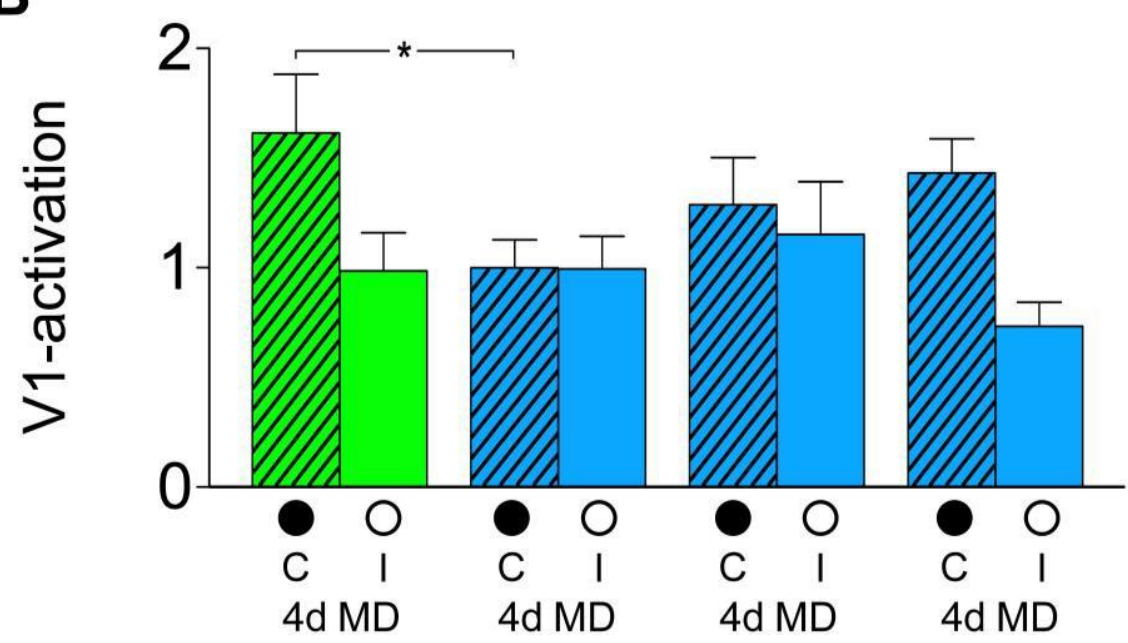

Figure 32: Ocular dominance indices and average response magnitudes of WT mice with injections of either AAV - shlc (control) $(n=4)$ or AAV - sh95 (knockdown of PSD-95) in the visual cortex of both hemispheres $(n=$ 7), only in the left hemisphere $(n=7)$, or in the right hemisphere $(n=5)$ after 4 days of MD. (A) Positive ODI values indicate dominance of the contralateral eye, negative ODI values indicate ipsilateral dominance. Halffilled circles represent ODI values of individual animals after 4 days of MD; means are marked by thick horizontal lines and the standard deviation is illustrated with small horizontal lines. Control mice (AAV - shlc, green) with 4 days of MD showed ocular dominance of the contralateral eye and positive ODI values after 4 days of MD. In contrast, WT mice with a knockdown of PSD-95 (blue) in both visual cortices showed a strong OD-shift towards zero which was significantly different from control mice (t-test, $\mathrm{p}<0.001$ ). Four days of monocular deprivation in WT mice with a knockdown of PSD-95 only in the left hemisphere (contralateral to the deprived eye), showed 
a strong OD-shift towards zero ( $p<0.001$; t-test), whereas mice with a knockdown in the right hemisphere did not show such OD-shift ( $p=0.2$, t-test). (B) Cortical response amplitudes in the visual cortex expressed as a change in reflectance $\times 10^{-4}$ after visual stimulation of the contralateral (C) or ipsilateral (I) eye in control (green) and PSD-95 knockdown mice (blue) after four days of MD. In control animals, cortical activation after visual stimulation of the contralateral eye was significantly higher than after ipsilateral eye stimulation $(t$-test, $p<$ 0.01), reflecting dominance of the contralateral eye in mice. In WT mice with a knockdown of PSD-95 either in both or only in the left visual cortex after four days of MD, the response amplitudes of the two eyes were no longer significantly different. Deprived eye responses were significantly reduced compared to controls ( $t$-test, $p$ < 0.05). In contrast to that, WT mice with a knockdown of PSD-95 only in the right hemisphere still exhibited higher visual cortical responses after stimulation of the contra eye than after stimulation of the ipsi eye ( $t$-test, $p$ $>0.05)$.

According to these data, a knockdown of PSD-95 only in the visual cortex contralateral to the deprived eye was sufficient to induce juvenile-like OD-plasticity (Huang et al., 2015). These data indicate that OD-plasticity and the reduction of contralateral eye responses in V1 are confined to the contralateral visual cortex.

Afterwards, it was tested whether it is possible to reverse the maturation of excitatory synapses in V1 and therefore restore juvenile-like OD-plasticity by silencing PSD-95 in WT mice shortly after the critical period for OD-plasticity (see: chapter 3.5).

\subsection{AAV transduction analysis of V1-specific PSD-95 knockdown and control mice (P 0)}

After optical imaging of intrinsic signals, animals were transcardially perfused and the brains sliced and mounted on glass slides. Confocal images were acquired with a Carl Zeiss LSM 710 microscope with a $10 \times$ objective to analyze transfection efficiency of the control virus (AAV shlc) or PSD-95 knockdown (AAV - sh95) mice (Figure 33). 


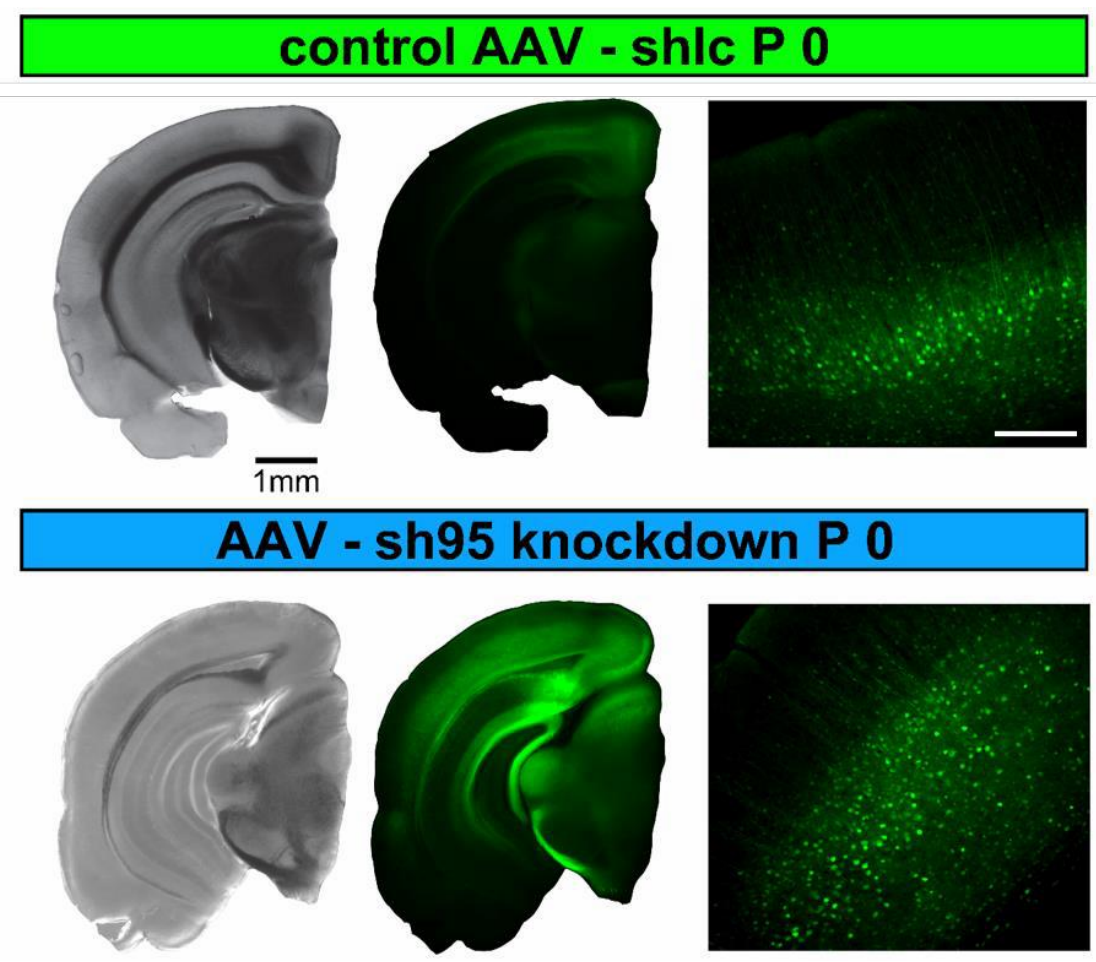

Figure 33: Analysis of AAV transduction rate from confocal brain images of AAV-shlc control and AAV-sh95 of P $\mathbf{0}$ transduced neurons. GFP fluorescence was captured for cellular level analysis. (Scale bar, $200 \mu \mathrm{m}$ ). Images were acquired by Huang (Schlüter laboratory, ENI, Göttingen).

\subsection{V1-specific knockdown of PSD-95 expression after maturation (P 40) restores juvenile-like OD-plasticity}

After the critical period (P 40), PSD-95 was knocked down (AAV - sh95), or controls (AAV shlc) selectively in the visual cortex of both hemispheres. Optical imaging of intrinsic signals was performed after 4 days of MD at $\sim P 80$ in both groups (Huang et al., 2015).

After four days of MD, control injected (AAV - shlc) animals showed a stronger visual cortical activation after visual stimulation of the contra eye than after visual stimulation of the ipsi eye, demonstrating and unchanged ocular dominance of the contralateral eye in the binocular region of $\mathrm{V} 1$. The ocular dominance indices were positive, indicating contralateral dominance and the representative 2-dimensional color coded ocular dominance maps in the binocular part of the left V1 showed warm, red colors (Figure 34 A, B).

In contrast to that, animals with a visual cortex restricted knockdown of PSD-95 in both hemispheres showed a weakening of visual cortical activation after visual stimulation of the contra eye after 4 days of MD, demonstrating OD-plasticity and ocular dominance of the 
ipsilateral eye in the binocular region of V1. The ocular dominance indices were negative, and the 2-dimensional color coded ocular dominance maps showed cold, blue and green colors. The histogram of $(C-I / C+I)$ was shifted to the left (Figure $34 \mathrm{C}, \mathrm{D})$.

\section{WT shlc control P40 after 4 days of MD}

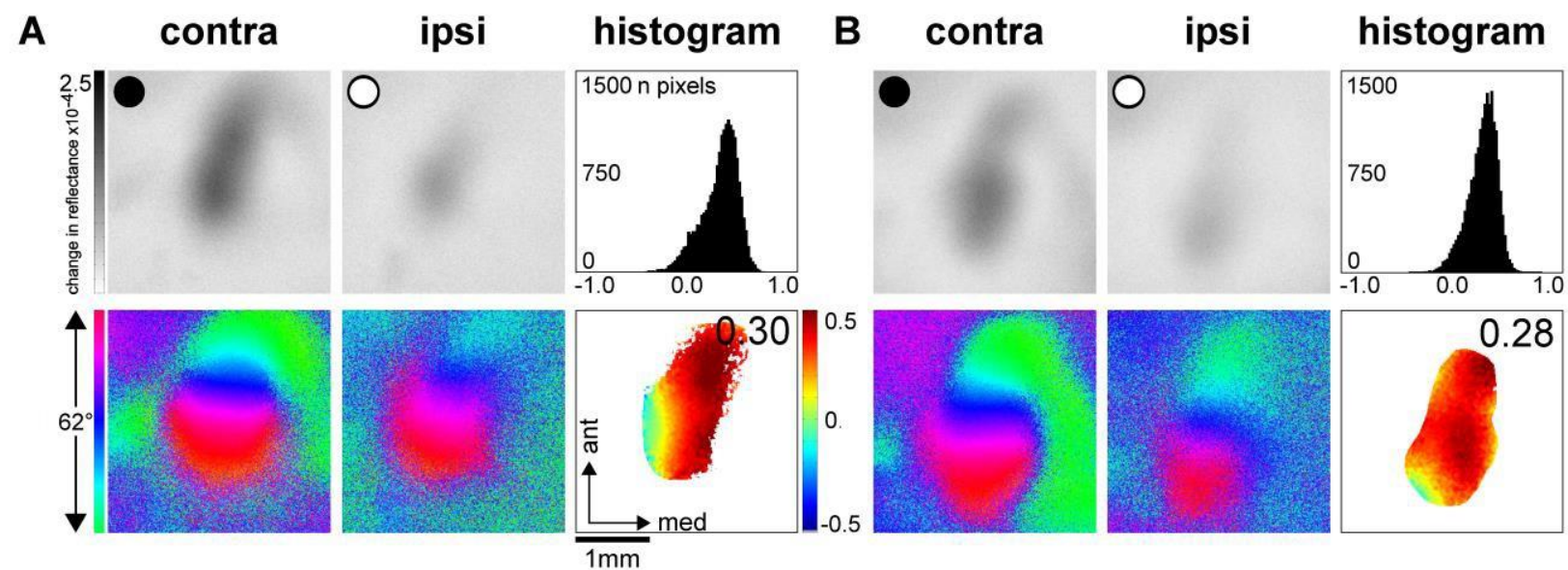

WT sh95 knockdown P40 after 4 days of MD

C

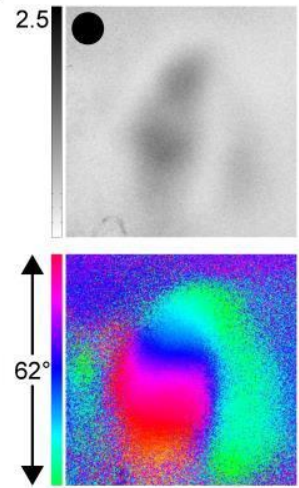

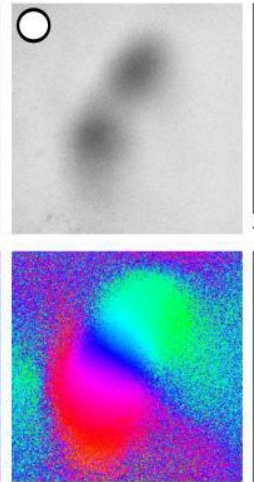

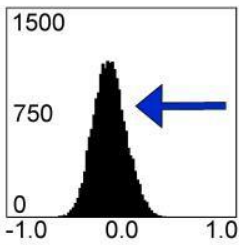

D
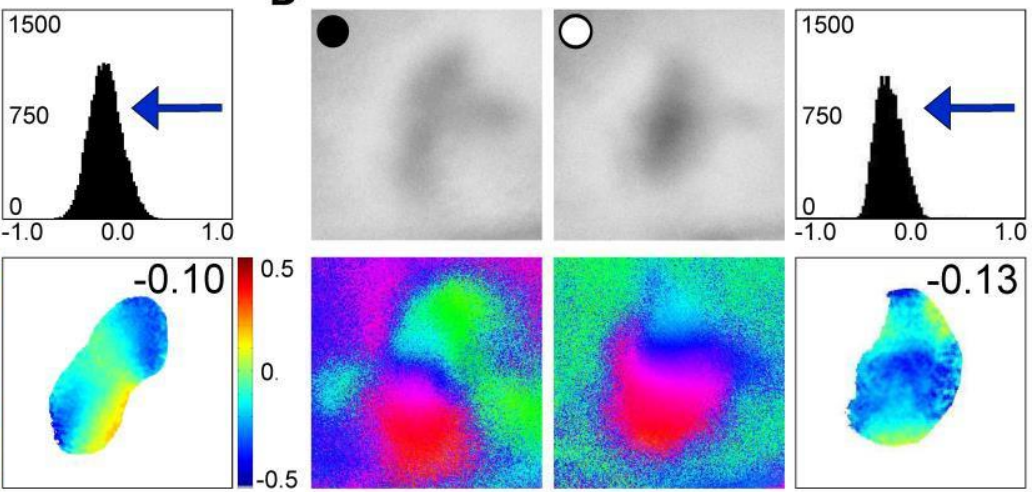

Figure 34: Representative examples of ocular dominance maps and indices in WT mice with injections of AAV shlc (control) or AAV - sh95 (PSD-95 knockdown) in V1 at P 40 after 4 days of MD obtained with optical imaging of intrinsic signals in vivo. Optical imaging maps of contralateral and ipsilateral cortical responses to visual stimulation of either eye with a horizontal moving bar (elevation) of $20^{\circ}$ are displayed from (A, B) two WT control mice (AAV - shlc) and (C, D) two WT mice with a visual cortex restricted knockdown of PSD-95 after 4 days of MD, respectively. Grayscale coded response magnitude maps (top rows, expressed as fractional change in reflectance $\times 10^{-4}$ ) with black circles indicating monocular deprivation of the eye and white circles indicating that the respective eye remained open. Color-coded phase maps of retinotopy (bottom rows), histograms of the OD-scores (top right of panels) and color-coded OD-maps (right bottom including the average ODI value), are illustrated. After 4 days of MD, control mice showed activity patches which were stronger after visual stimulation of the contra eye than after stimulation of the ipsi eye. Positive ODI values and warm, red colors prevailed in the according color coded 2-dimensional OD-map, indicating contralateral dominance. In contrast to that, mice with a knockdown of PSD-95 exhibited a strong OD-shift towards zero. Activity patches evoked by stimulation of the 
contra eye was equally strong to that after ipsi eye stimulation. The histogram shifted to the left, the average ODI was negative, and cold, blue colors prevailed in the 2-dimensional OD-map. Hence, indicating ipsilateral dominance and ocular dominance plasticity.

Quantification of the data revealed that WT mice transduced with AAV - sh95 showed a juvenile-like OD-shift after 4 days of MD (ODI: $-0.02 \pm 0.02 ; n=10)$, whereas control mice (AAV - shlc) did not show OD-plasticity (ODI: $0.25 \pm 0.01 ; n=8 ; p<0.001 ;$ t-test) (Figure 35).
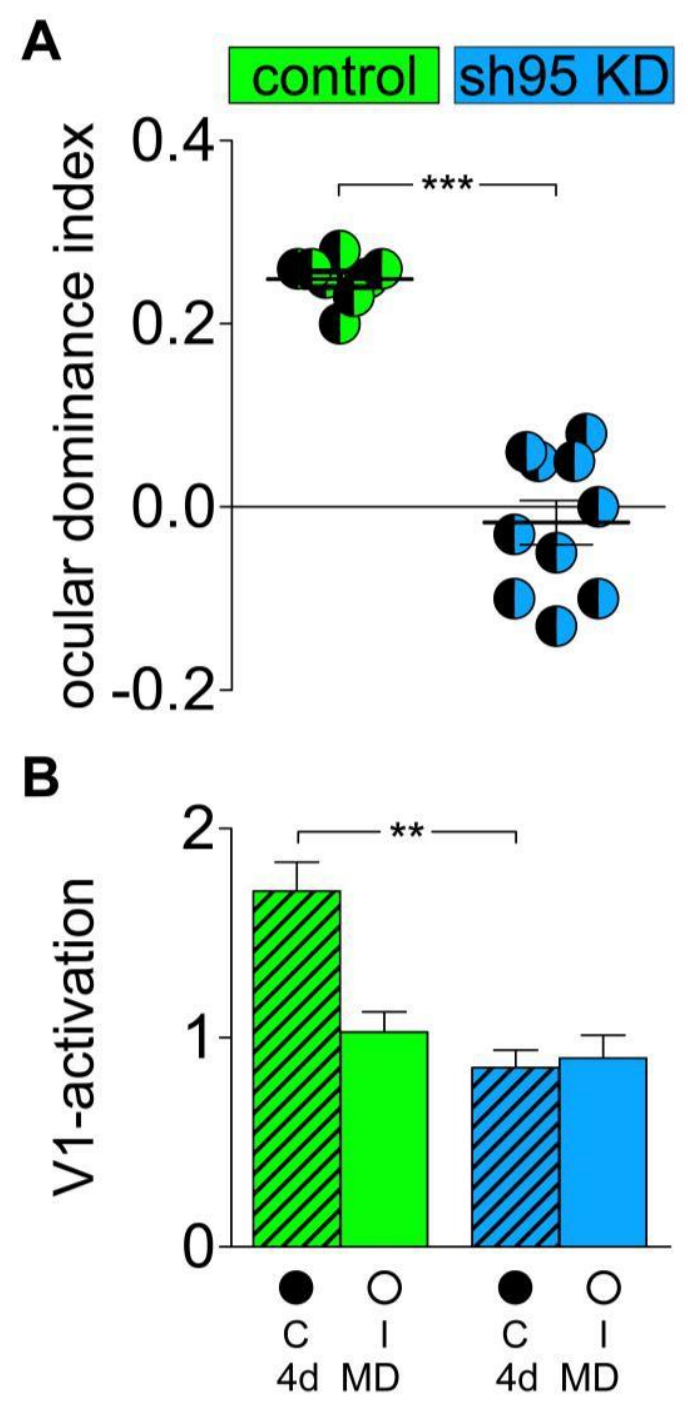

Figure 35: Ocular dominance indices and average response magnitude of WT mice with injections of either AAV - shlc (control, $n=8$ ) or AAV - sh95 (knockdown of PSD-95, $n=10$ ) in the visual cortex of both hemispheres after 4 days of MD. (A) Positive ODI values indicate dominance of the contralateral eye, whereas negative ODI values indicate ipsilateral dominance. Half-filled circles represent ODI values of individual animals after 4 days of MD; means are marked by thick horizontal lines and the standard deviation is illustrated with small horizontal lines. Control mice (AAV - shlc, green) with 4 days of MD showed ocular dominance of the contralateral eye and positive ODI values. In contrast, mice with a knockdown of PSD-95 (blue) showed a strong OD-shift towards zero which was significantly different from control injected mice (t-test, $p<0.001)$. (B) Cortical response amplitudes 
in the visual cortex expressed as a change in reflectance $\times 10^{-4}$ after visual stimulation of the contralateral (C) or ipsilateral (I) eye in control mice (green) and PSD-95 knockdown mice (blue) after four days of MD. In control animals, cortical activation after visual stimulation of the contralateral eye was significantly higher than after ipsilateral eye stimulation ( $t$-test, $p<0.001$ ), reflecting the dominance of the contralateral eye in mice. In WT mice with a knockdown of PSD-95 after four days of MD, the response amplitudes of the two eyes were no longer significantly different. The deprived eye responses were significantly reduced compared to controls ( $t$ test, $\mathrm{p}<0.01)$.

\subsection{AAV transduction analysis of V1-specific PSD-95 knockdown and control mice (P} 40)

After optical imaging of intrinsic signals, animals were transcardially perfused and the brains sliced and mounted on glass slides. Confocal images were acquired with a Carl Zeiss LSM 710 microscope with a $10 \times$ objective to analyze transfection efficiency of the control virus (AAV shlc) or PSD-95 knockdown (AAV - sh95) mice (Figure 36). 

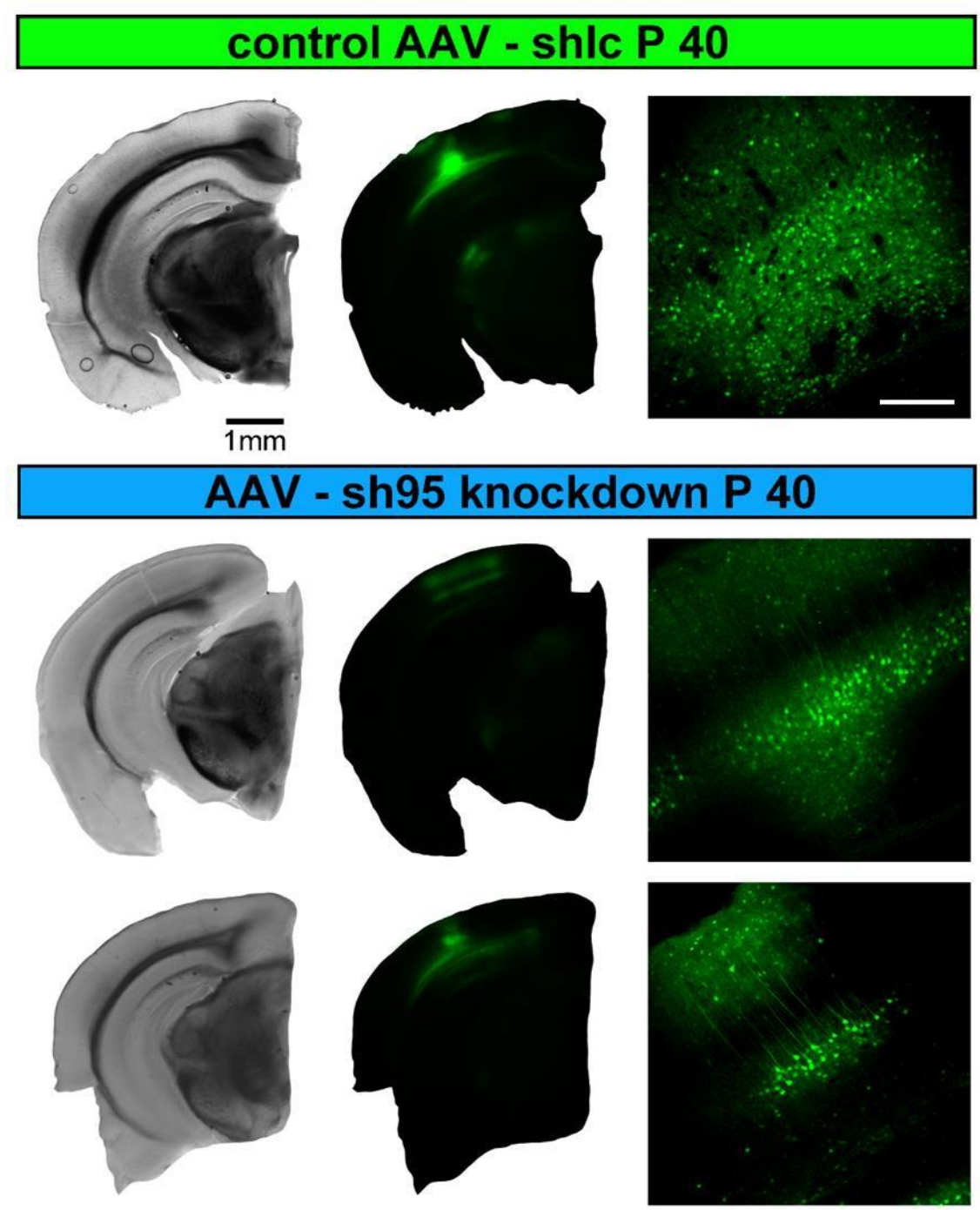

Figure 36: Analysis of AAV transduction rate from confocal brain images of AAV - shlc control and AAV - sh95 of P 40 transduced neurons. GFP fluorescence was captured for cellular level analysis. (Scale bar $200 \mu \mathrm{m}$ ). Images were acquired by Huang (Schlüter laboratory, ENI, Göttingen).

\subsection{Normal visual acuity and orientation discrimination before and after a selective knockdown of PSD-95 in the visual cortex of WT mice}

It was investigated whether a knockdown of PSD-95 in the VC of mice (P 0) would result in a similar phenotype as observed in mice with a global knockout of the protein. PSD-95 KO mice exhibit normal visual acuity (Huang et al., 2015; see: Figure 38 A) but impaired orientation discrimination (Figure 38 B) which was tested in the visual water task (Götze and Wenzel; Löwel laboratory, Göttingen).

WT mice ( P 60) that were either transduced with AAV - sh95 (PSD-95 knockdown) or AAV shlc serving as controls in both VC were tested in the visual water task (Prusky, 2000), which 
is a behavioral task based on reinforcement learning (Figure $37 \mathrm{~A}$ ). Both visual acuity and orientation discrimination was examined.

Mice with a knockdown of PSD-95 in both VC showed normal visual acuity and had similar values to that of control injected (AAV - shlc) mice (AAV - sh95: $0.51 \pm 0.01$ cycles/degree (cyc/deg); $n=4 ; A A V$ - shlc: $0.54 \pm 0.02 ; n=5 ; p=0.23 ; t$-test). The values were comparable to that of WT and PSD-95 KO mice (PSD-95 KO: $0.51 \pm 0.03 \mathrm{cyc} / \mathrm{deg}, \mathrm{n}=8 ; \mathrm{p}=0.95$, t-test; WT: $0.56 \pm 0.04 \mathrm{cyc} / \mathrm{deg}, \mathrm{n}=8, \mathrm{p}=0.4$; t-test) (Figure $38 \mathrm{~B}$ ).

Likewise, orientation discrimination was similar in PSD-95 knockdown and control injected mice (AAV - sh95: $15 \pm 3.3^{\circ} ; n=5 ; A A V-$ shlc: $14.6 \pm 1.3 ; n=4 ; p=0.94 ; t$-test; Figure $37 \mathrm{C}$ ).

A

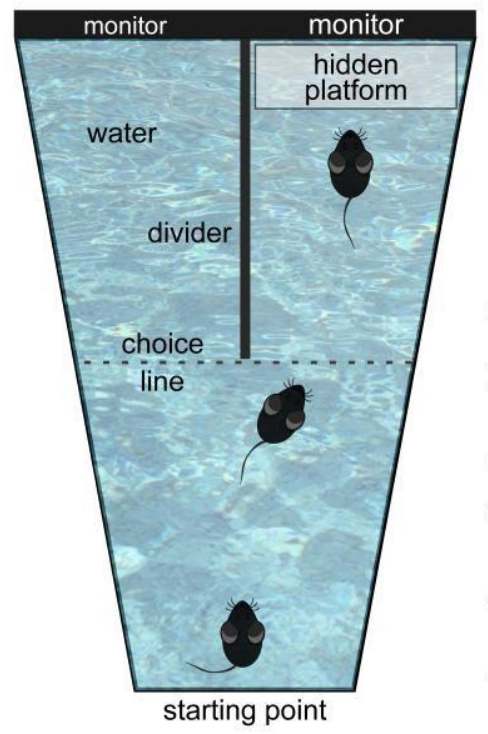

B

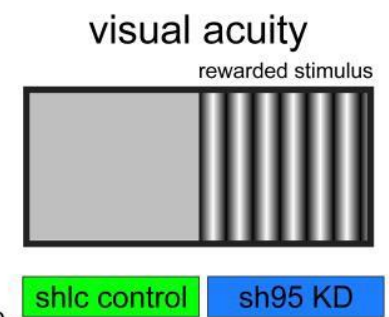

C

\section{orientation discriminaton}

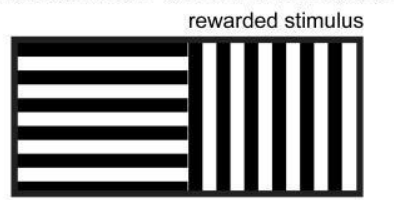

shlc control sh95 KD

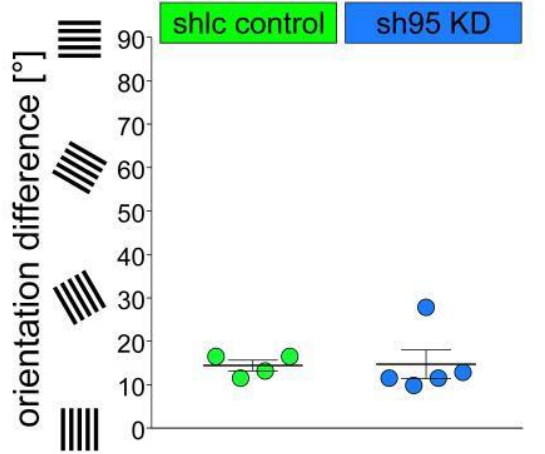

Figure 37: Maximum spatial frequency threshold and minimum angle difference of WT mice with VC restricted injections of AAV - shlc (control) or AAV - sh95 (knockdown of PSD-95) at PO - P2; determined in the visual water task (Prusky, 2000). Maximally perceived spatial frequency (visual acuity) and orientation discrimination of WT mice injected with AAV - shlc (control; $n=4$ ) or a knockdown of PSD-95 (AAV - sh95; $n=5$ ) in the visual cortex. (A) Schematic overview of the visual water task which allows testing of both visual acuity and orientation discrimination of mice. (B) To determine visual acuity, mice are trained to swim towards a monitor screen displaying a stationary, sinusoidal grating of black and white bars, while isoluminent gray is displayed on the reference screen. Visual acuity of mice with a knockdown of PSD-95 in the visual cortex was similar to that of control mice ( $p=0.23$, t-test). (C) To test orientation discrimination mice are trained to distinguish a horizontal grating from a vertical grating. In front of the stimulus screen, a hidden platform is placed underneath the water surface serving as a reward. Orientation discrimination was not impaired and similar in both groups $(p=0.94, t-$ test). Each data point indicates the mean of 3 values obtained from each animal. Bars represent mean \pm SEM. 
Orientation discrimination of PSD-95 knockdown and control injected mice was comparable to values from that of WT mice (WT: $21 \pm 3^{\circ} ; n=7 ; p=0.2$; t-test) (Figure 38 A). However, they were significantly different from values obtained from PSD-95 KO mice (PSD-95 KO: $51 \pm$ $4.6^{\circ} ; n=11 ; p<0.001 ; t$-test) (Figure 38 B). PSD-95 KD mice showed significantly better orientation discrimination than ubiquitous PSD-95 KO mice which were clearly impaired (PSD$95 \mathrm{KO}: 51.2 \pm 4.6^{\circ} ; \mathrm{n}=11 ; \mathrm{p}<0.001 ; \mathrm{t}$-test) (Figure $38 \mathrm{~B}$ ).

A
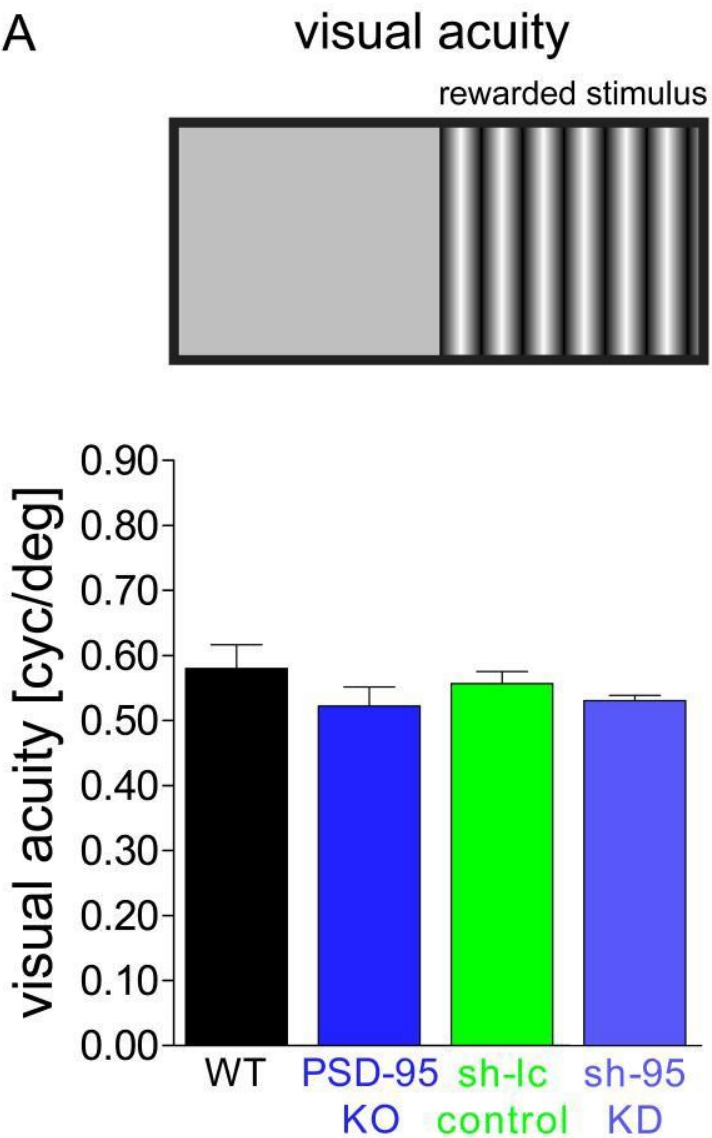

B orientation discrimination rewarded stimulus
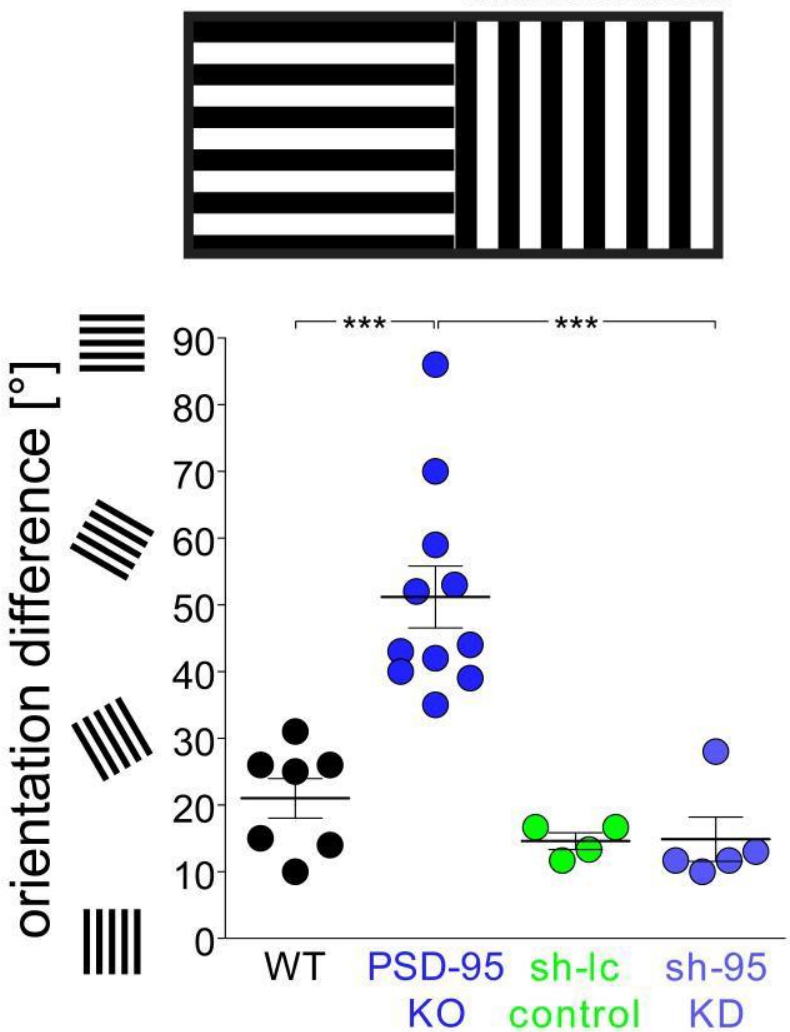

Figure 38: Maximum spatial frequency threshold and minimum angle difference of WT, PSD-95 KO, WT AAV shlc and PSD-95 KD mice determined in the visual water task (Prusky, 2000). (A) Maximally perceived spatial frequency (visual acuity) of WT mice $(n=8)$, PSD-95 KO mice $(n=8)$ and WT mice injected with AAV - shlc (control; $n$ $=4$ ) or a knockdown of PSD-95 (AAV - sh95; $n=5$ ) in the visual cortex. Visual acuity (cyc/deg) was similar in all tested groups ( $p>0.05$; t-test). (B) Orientation discrimination of WT mice $(n=7)$, PSD-95 KO mice $(n=11)$ and WT mice injected with AAV - shlc (control; $n=4$ ) or a knockdown of PSD-95 (AAV - sh95; $n=5$ ) in the visual cortex. Orientation discrimination was clearly impaired in PSD-95 KO mice compared to that of WT mice ( $p<$ 0.001, t-test) and significantly different in comparison to PSD-95 knockdown mice ( $<<0.001, t$-test). WT mice either injected with AAV -shlc (control) or AAV - sh95 (PSD-95 KD) in the visual cortex obtained similar values to that of WT mice ( $p>0.05$ for all comparisons, t-test). Each data point indicates the mean of 3 values from each animal. Bars represent mean \pm SEM. (WT and ubiquitous PSD-95 KO mice were tested by Götze and Wenzel, Löwel laboratory, ENI, Göttingen). 
To get better insights if a loss of PSD-95 only in the visual cortex might influence visual capabilities, visual acuity and orientation discrimination was also tested in WT mice before and after a knockdown of PSD-95 in the VWT.

WT mice (P $84-111 ; n=12)$ showed normal visual acuity $(0.58 \pm 0.01 \mathrm{cyc} / \mathrm{deg})$ and orientation discrimination $\left(11.6 \pm 0.94^{\circ}\right)$ measured in the VWT (Figure 39$)$ and both visual capabilities did not change after a knockdown of PSD-95 in the visual cortex neither for visual acuity (before injection: $0.57 \pm 0.02 \mathrm{cyc} / \mathrm{deg}$; after PSD-95 KD: $0.57 \pm 0.02 \mathrm{cyc} / \mathrm{deg}, \mathrm{n}=7 ; \mathrm{p}=0.3$, t-test) nor for orientation discrimination (before injection: $12.1 \pm 1.1^{\circ}$; after PSD-95 KD: $11.2 \pm 0.8^{\circ}, \mathrm{n}=$ 7; $p=0.6$, t-test) (Figure 39). Similarly, control animals injected with AAV - shlc also showed unaltered visual acuity (before injection: $0.58 \pm 0.02 \mathrm{cyc} / \mathrm{deg}$; after injection: $0.58 \pm 0.04$ cyc/deg, $n=5 ; p=0.8$, t-test) and orientation discrimination (before injection: $11 \pm 1.7^{\circ}$; after injection: $9.2 \pm 1.3^{\circ}, n=5 ; p=0.4$; t-test) before and after the injection (Figure 39). PSD-95 $K D$ and control injected animals had also similar visual acuity ( $p=0.9$; t-test) and orientation discrimination ( $p=0.2$; t-test). (VWT experiments were done by Lübben (Bachelor student) and Stodieck).

A
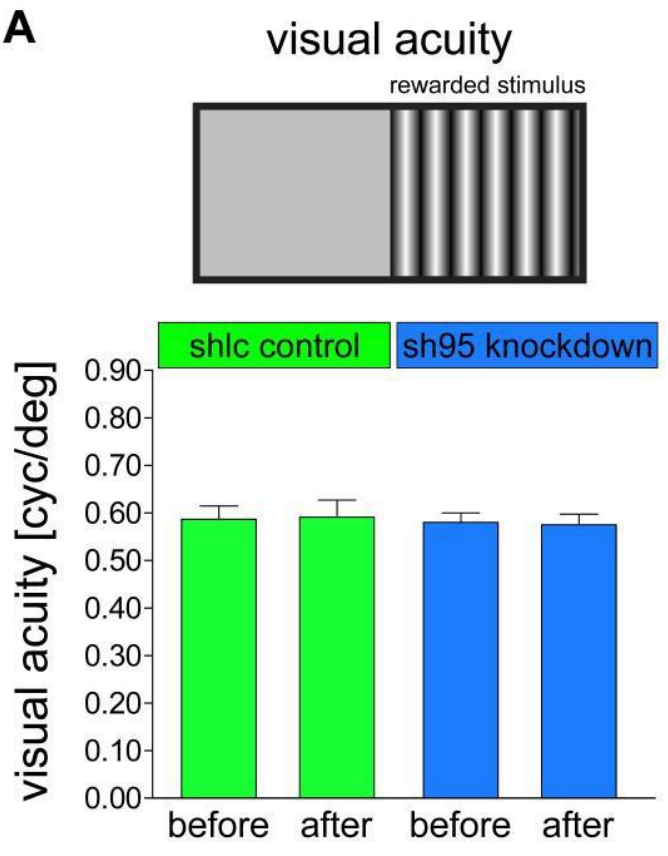

B
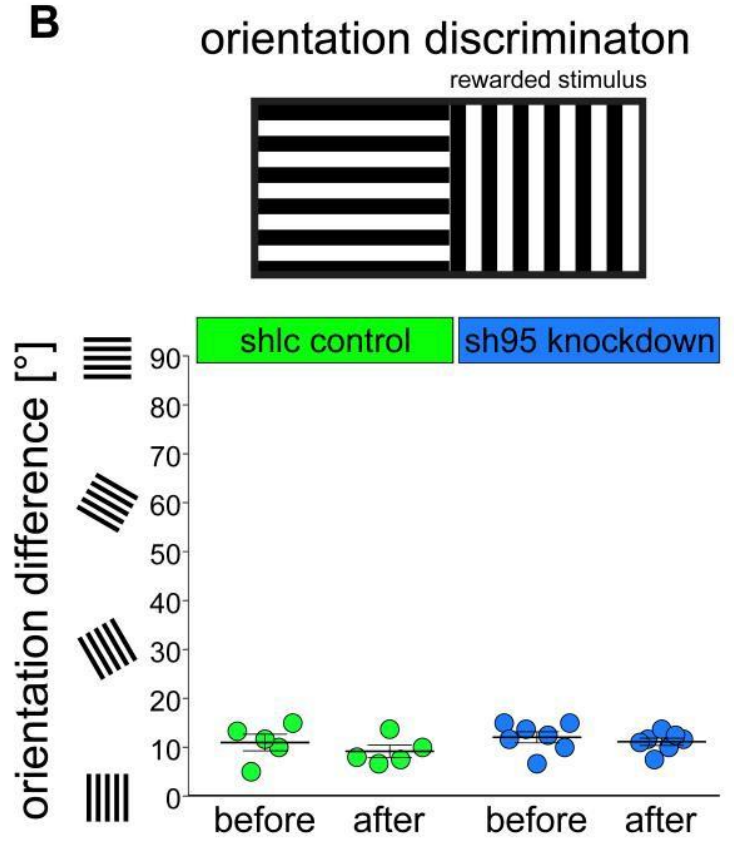

Figure 39: Maximum spatial frequency threshold and minimum angle difference determined in the visual water task in WT mice before and after a knockdown of PSD-95 in the visual cortex. Maximally perceived spatial frequency (visual acuity) and orientation discrimination of WT mice before and after injection with AAV - shlc (control; $n=5$ ) or a knockdown of PSD-95 (AAV - sh95; $n=7$ ) in the visual cortex. (A) Visual acuity of WT mice with a knockdown of PSD-95 in the visual cortex was similar to that of control mice ( $p=0.7, t$-test) and did 
not change after virus injection in the control group $(p=0.8)$ or in mice after knockdown of PSD-95 ( $p=0.3)$. (B) Likewise, orientation discrimination was not impaired after a visual cortex restricted knockdown of PSD-95 ( $p=$ $0.2, t$-test) and did not change in the respective groups before and after virus injection in the control group $(p=$ 0.38 , t-test) or in the knockdown group ( $p=0.55$, t-test). Each data point indicates the mean of 3 values obtained from each animal. Bars represent mean \pm SEM.

There are two possible explanations why there is a discrepancy between the orientation discrimination of ubiquitous PSD-95 KO mice and mice with a knockdown of PSD-95 only in the visual cortex. Either the transfection efficiency of the virus was not good enough to elicit a behavioral change or there could be other brain regions involved in which PSD-95 is responsible for normal orientation discrimination. However, this seems to be unlikely since testing the transfection efficiency in brain slices revealed no homogenous distribution of the virus in the visual cortex and variation between the different animals could be observed (see Figure 42). Furthermore, our collaborating group under the direction of Prof. Dr. Dr. Schlüter repeated these experiments and could indeed find impaired orientation discrimination in visual cortex restricted PSD-95 knockdown mice (preliminary data).

\subsection{V1-specific knockdown of PSD-95 expression after the phase for OD-plasticity (beyond P 110; Löwel \& Lehmann, 2008) restores juvenile-like OD-plasticity}

The same mice that were tested in the visual water task before and after virus injections with AAV - shlc or AAV - sh95 (> P 110), were also tested for OD-plasticity using optical imaging of intrinsic signals. In standard cage raised WT mice, OD-plasticity is gone after P 110. Hence, it was of interest to examine whether a visual cortex restricted knockdown of PSD-95 after the phase for OD-plasticity would also restore juvenile-like OD-plasticity as shown before in PSD95 knockdown mice $<$ P110.

Therefore, optical imaging of intrinsic signals was performed in control injected mice (AAV shlc) and PSD-95 knockdown mice (AAV - sh95) after 4 days of MD (P 182 - 215) and after 7 days of $M D(P 185-218)$ in the same animals.

After 4 days of MD, control injected (AAV - shlc) animals showed a stronger visual cortical activation after visual stimulation of the contra eye than after visual stimulation of the ipsi eye, demonstrating unchanged ocular dominance of the contralateral eye in the binocular 
region of V1 (Figure $40 \mathrm{~A}$ ). The ocular dominance indices were positive indicating contralateral dominance and the representative 2-dimensional color coded ocular dominance maps in the binocular part of the left V1 showed warm, red colors (Figure $40 \mathrm{~A}$ ). This did not change after 7 days of MD after which the binocular region of the V1 was still dominated by inputs coming from the contralateral eye and showed a higher cortical activation after contra eye stimulation than after ipsi eye stimulation. Similarly, the ocular dominance indices were positive and the 2-dimensional color coded ocular dominance maps in the binocular part of the left V1 showed warm, red colors indicating contralateral dominance (Figure $40 \mathrm{~B}$ ).

In contrast to that, WT mice with a visual cortex restricted knockdown of PSD-95 in both hemispheres showed stronger visual cortical activation after visual stimulation of the ipsi eye than after contra eye stimulation after 4 days of MD (Figure $40 \mathrm{C}$ ) as well as after 7 days of MD (Figure 40 D). Hence, demonstrating OD-plasticity and ocular dominance of the ipsilateral eye in the binocular region of V1. The ocular dominance indices were negative and the 2dimensional color coded ocular dominance maps showed cold, blue and green colors and the histogram of $(C-1 / C+I)$ was shifted to the left (Figure $40 \mathrm{C}, \mathrm{D})$. 
after $4 \mathrm{~d}$ of MD

\section{WT shlc control}

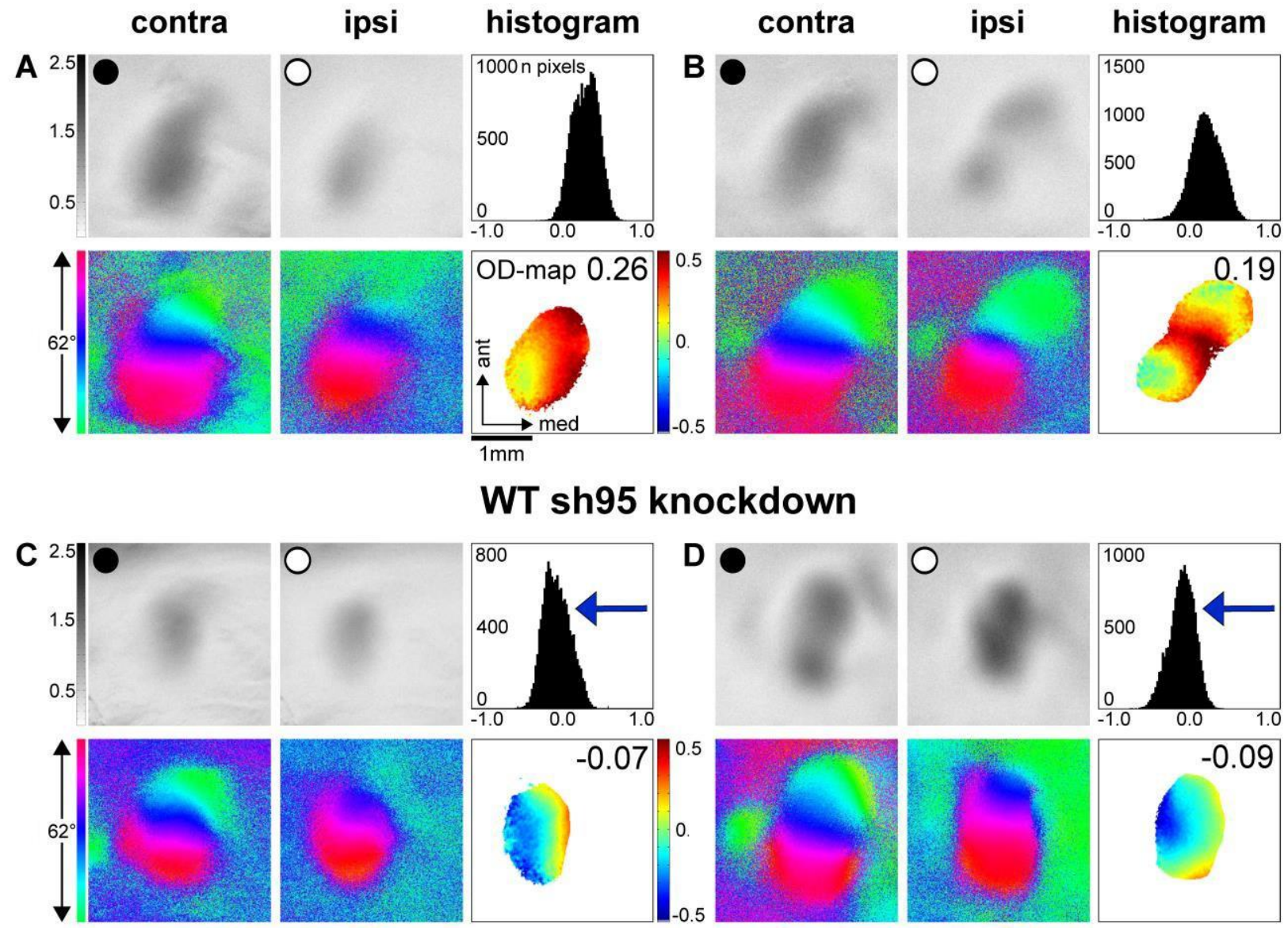

Figure 40: Representative examples of ocular dominance maps and indices in WT mice injected with a control virus (AAV - shlc) or a knockdown of PSD-95 (AAV - sh95) in the visual cortex after 4 and after 7 days of MD (> P 110) obtained with optical imaging of intrinsic signals in vivo. Optical imaging maps of contralateral and ipsilateral cortical responses to visual stimulation of either eye with a horizontal moving bar (elevation) of $20^{\circ}$ are displayed from (A, B) a control mouse (AAV - shlc) and (C, D) a mouse with a visual cortex restricted knockdown of PSD-95 after 4 and 7 days of MD, respectively. Grayscale coded response magnitude maps (top rows, expressed as fractional change in reflectance $\times 10^{-4}$ ) with black circles indicating monocular deprivation of the eye and white circles indicating that the respective eye remained open. Color-coded phase maps of retinotopy (bottom rows), histograms of the OD-scores (top right of panels) and color-coded OD-maps (right bottom including the average ODI value), are illustrated. (A) After 4 days as well as after 7 days of MD (B) in WT control mice, the activity patches evoked by stimulation of the contra eye were always darker than those evoked by ipsi eye stimulation. The average ODI was always positive and warm, red colors prevailed in the 2-dimensional OD-map, indicating contralateral dominance. In contrast to that, (C) 4 days and (D) 7 days of MD in WT mice with a knockdown of PSD-95 induced an OD-shift towards zero. Activity patches evoked by stimulation of the contralateral (contra) eye were equally strong to that after ipsi eye stimulation, the ODI histogram shifted to the left, the average ODI was negative and cold, blue colors prevailed in the 2-dimensional OD-map. Hence, indicating ipsilateral dominance and ocular dominance plasticity. 
These data reveal that even a late knockdown of PSD-95 renders mice plastic in the VC after 4 days of MD (ODI: $-0.05 \pm 0.04, n=6$ ) as well as after 7 days of MD (ODI: $0.04 \pm 0.04, n=6 ; p$ $=0.1$; t-test). In contrast, age-matched controls (AAV - shlc) were still dominated by sensory inputs coming from the contralateral eye and values were significantly different from mice with a PSD-95 KD after either 4 days of MD (ODI: $0.27 \pm 0.03, n=5 ; p<0.001 ; t$-test) and also after 7 days of $\mathrm{MD}$ (ODI: $0.23 \pm 0.03, n=3 ; p<0.05$; t-test) (Figure 41).

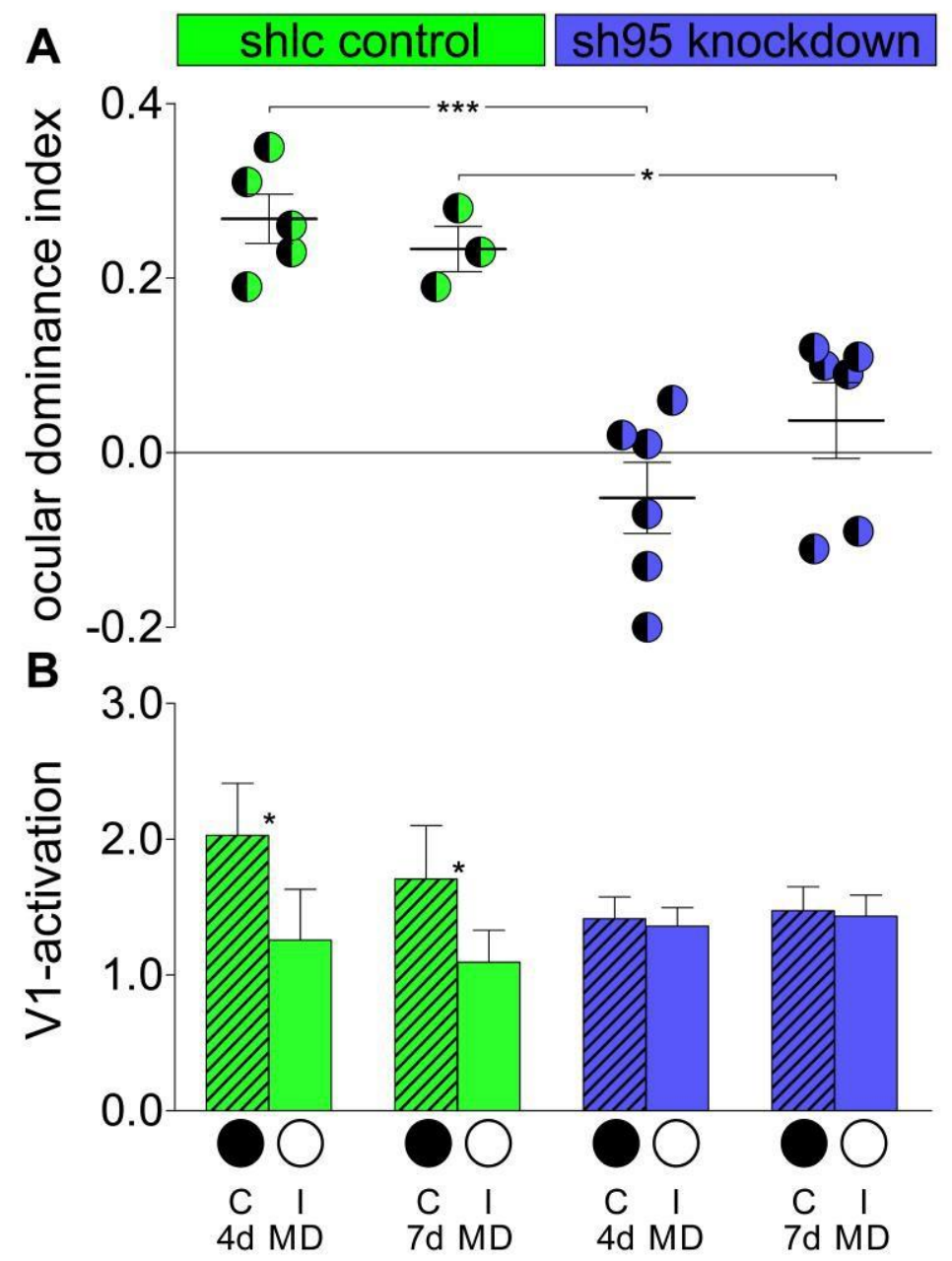

Figure 41: Ocular dominance indices and maximum response magnitude of WT mice with injections of either AAV - shlc (control, $n=5$ ) or AAV - sh95 (knockdown of PSD-95, $n=7$ ) in the visual cortex of both hemispheres after 4 and 7 days of MD. (A) Positive ODI values indicate dominance of the contralateral eye, whereas negative ODI values indicate ipsilateral dominance. Half-filled circles represent ODI values of individual animals after 4 or 7 days of MD; means are marked by thick horizontal lines and the standard deviation is illustrated with small horizontal lines. Control mice (AAV - shlc, green) after 4 and 7 days of MD showed ocular dominance of the contralateral eye and positive ODI, whereas mice with a knockdown of PSD-95 (blue) showed a strong OD-shift towards zero which was significantly different from control injected mice after 4 days $(p<0.001$, t-test) and also after 7 days of MD ( $p<0.05$, t-test). (B) Cortical response amplitudes in the visual cortex expressed as a change 
in reflectance $\times 10^{-4}$ after visual stimulation of the contralateral (C) or ipsilateral (I) eye in control mice (green) and PSD-95 knockdown mice (blue) after 4 or 7 days of MD. In control animals, cortical activation after visual stimulation of the contralateral eye was significantly higher than after ipsilateral eye stimulation $(p<0.05$, paired ttest), reflecting the dominance of the contralateral eye in mice. In WT mice with a knockdown of PSD-95 after 4 and 7 days of MD, the response amplitudes of the two eyes were no longer significantly different after 4 days ( $p=$ 0.9 , paired t-test) and after 7 days of $M D(p=0.7$, paired t-test).

These results indicate that a lack of PSD-95 only in the visual cortex is already sufficient to induce OD-plasticity in WT mice. The results suggest that PSD-95 is regulating the maturation of excitatory synapses and is required for the maturation and the stabilization of cortical networks. A knockdown of PSD-95 contralateral to the deprived eye was already sufficient to trigger juvenile-like OD-plasticity even after the CP (Huang et al., 2015) and beyond the phase for OD-plasticity above P 110 (unpublished data). 


\subsection{AAV transduction analysis of V1-specific PSD-95 knockdown and control mice (> P} 110)

After optical imaging of intrinsic signals, animals were transcardially perfused and in situ pictures were taken using a Sony alpha 6000 camera and a BlueStar Nightsea flashlight with yellow filter glasses to excite GFP fluorescence of the specimen (Figure 42).

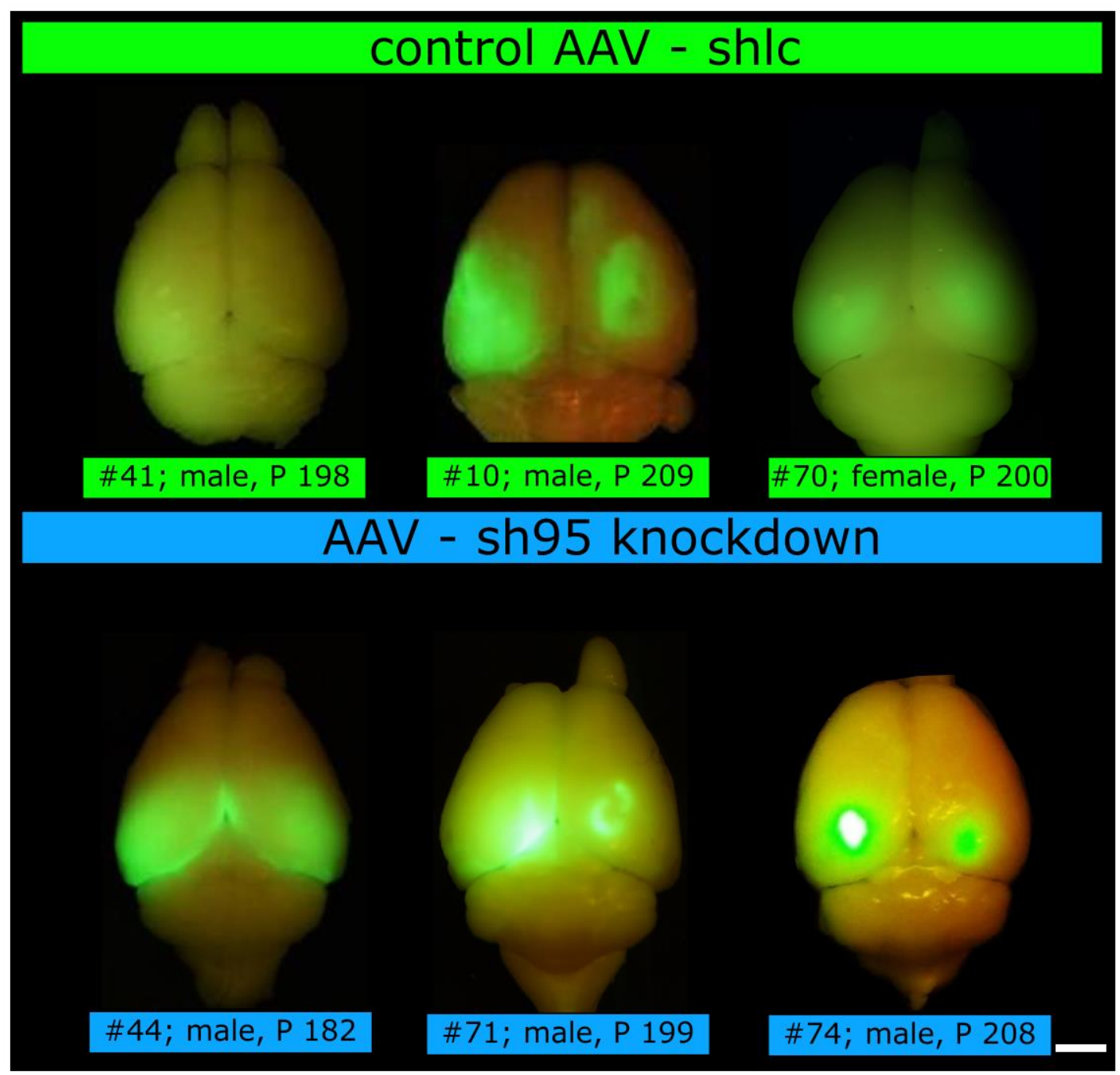

Figure 42: In situ brain images of virus injections of either AAV - shlc control or AAV - sh95 in > P 110 injected WT mice. Brain images were taken from above to illustrate GFP fluorescence in control animals (AAV - shlc) and PSD-95 knockdown animals (AAV - sh93). Scale bar $1 \mathrm{~mm}$. 
The brains were then sliced and mounted on glass slides. Confocal images were acquired with a Nikon SMZ18 microscope to analyze transfection efficiency of the control virus (AAV - shlc) or PSD-95 knockdown (AAV - sh93) mice (Figure 43).

The transfection efficiency in brain slices revealed no homogenous distribution of the virus in the visual cortex and variation between the different animals could be observed.

All brain sections were transfected with the respective virus and revealed GFP fluorescence. However, the transfection efficiency in brain slices revealed no homogenous distribution of the virus in the visual cortex and variation between the different animals could be observed. 


\section{AAV-shlc control}

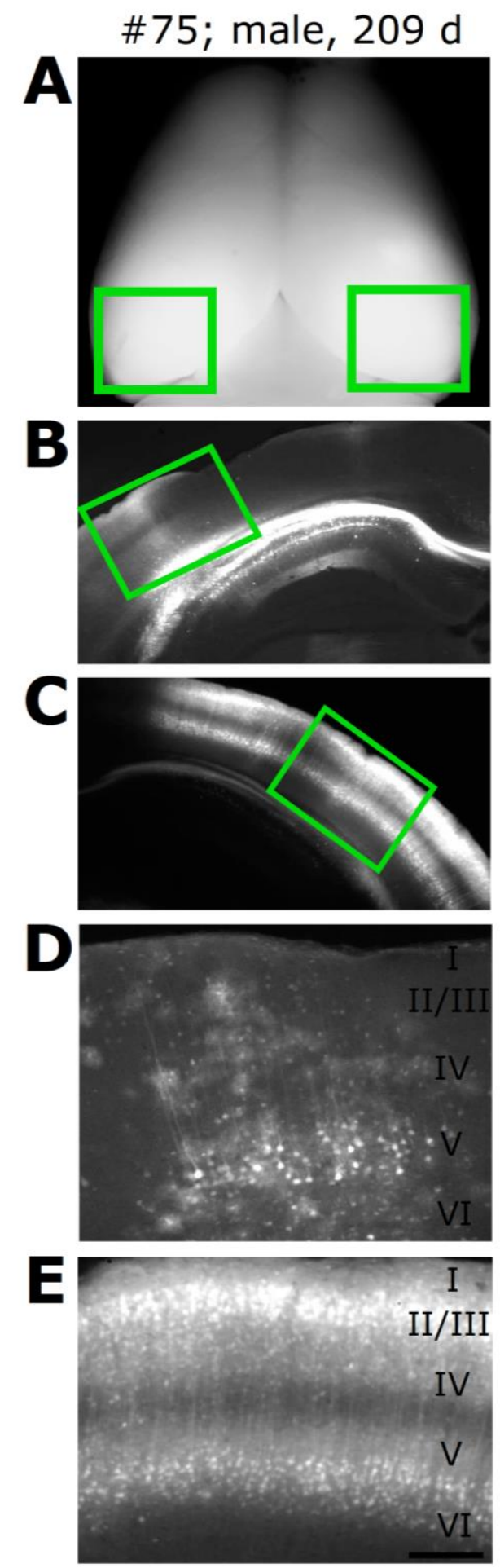

\section{AAV-sh95 KD}
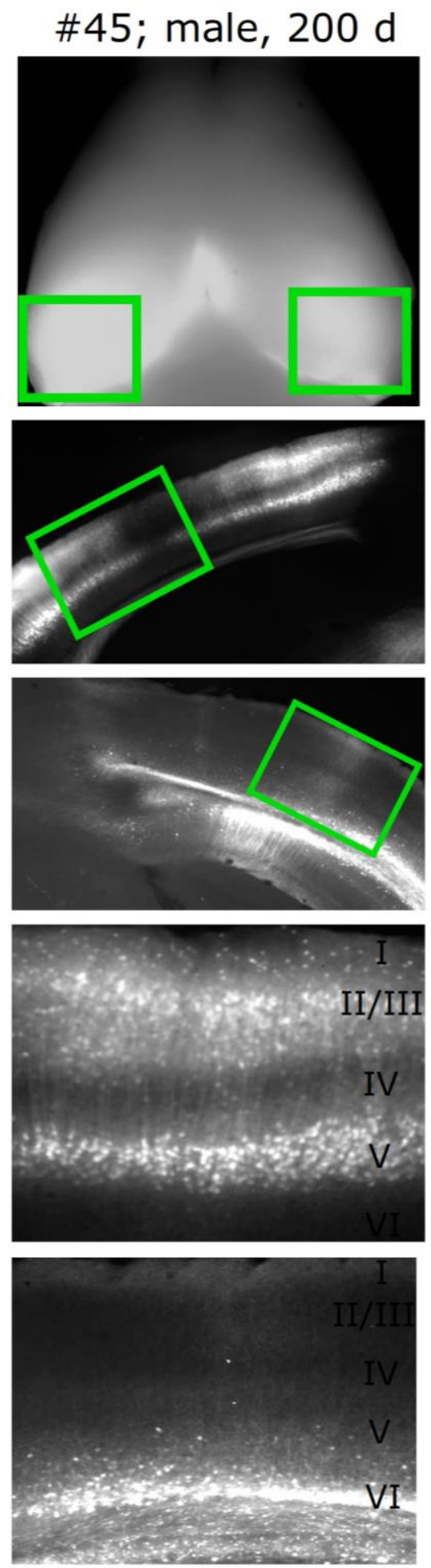

Figure 43: Microscopy images of virus transduction of either AAV - shlc control or AAV - sh95 WT mice injected after > P 110. (A) Brain images taken from above to illustrate GFP fluorescence in control animals (AAV - shlc) or PSD-95 knockdown animals (AAV - sh95). (B) Images obtained from brain slices of the left hemisphere from either control animals (left column) or PSD-95 knockdown mice (right column) and (C) the respective right hemisphere. Green rectangles represent the area of the visual cortex of the respective hemisphere. (D) Images of the visual cortex of either control animals (left column) or PSD-95 knockdown animals (right column) of the left hemisphere or (E) the right hemisphere, respectively. Scale bar $200 \mu \mathrm{m}$. 
Transfection efficiency was determined by the level of fluorescence of the visual cortex from microscopy images obtained from all animals (AAV - shlc controls and AAV - sh95 knockdowns) and analyzed with the program ImageJ. Fluorescence intensity was determined by the mean gray value of each visual cortex that was subtracted from background noise. Analysis with ImageJ revealed that the transfection efficiency was higher in AAV - shlc control animals than in AAV sh95 knockdown mice (Figure 44).

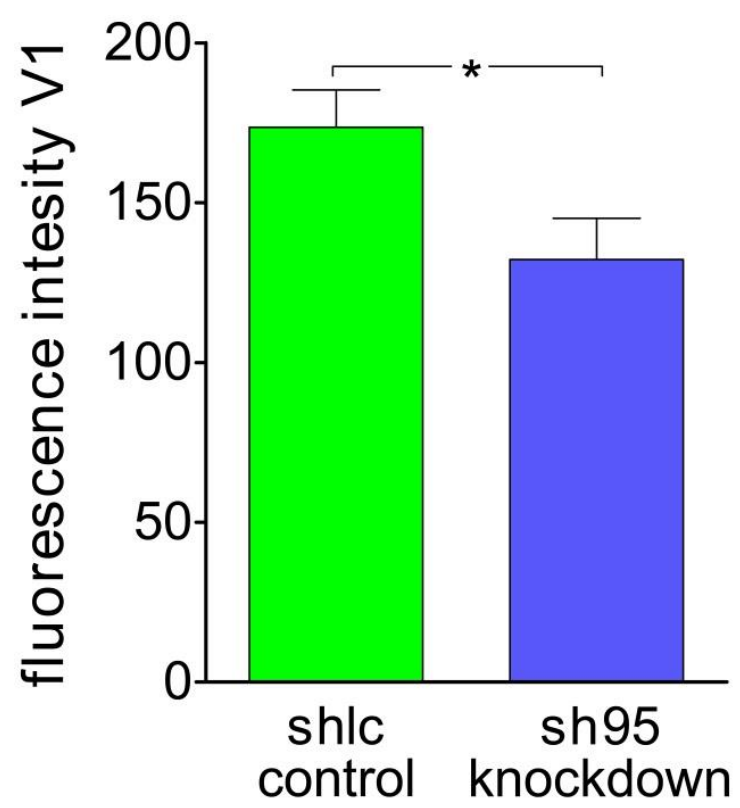

Figure 44: Fluorescence intensity measured in the visual cortex of AAV - shlc control and AAV - sh95 injected WT animals at > P 110. Fluorescence intensities in the visual cortex of control injected animals (AAV - shlc) were higher in comparison to PSD-95 knockdown animals (AAV - sh95) $(p=0.04)$.

However, there was no statistical difference when comparing transfection efficiency in the visual cortex and obtained ocular dominance indices in the respective animals of either AAV shlc control or AAV - sh95 knockdown animals (Figure 45). 


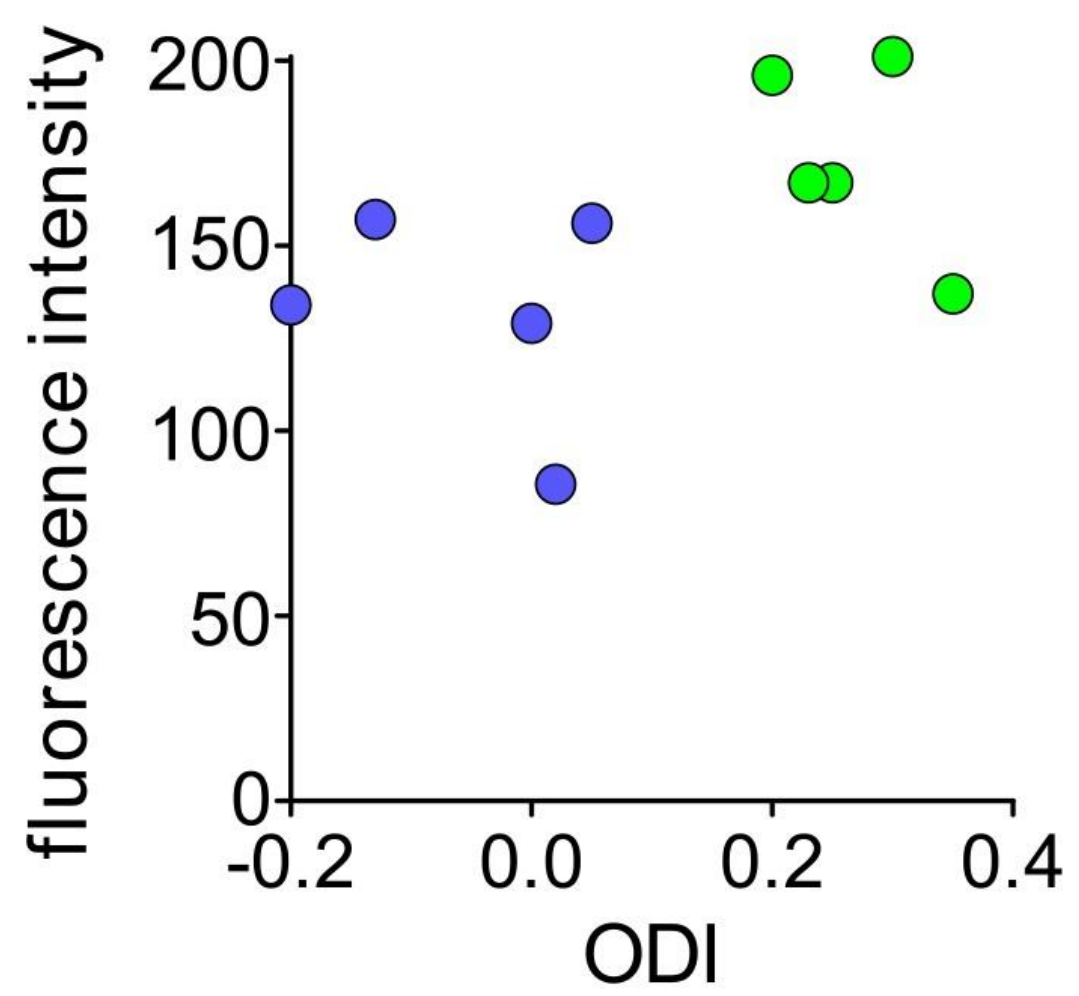

Figure 45: Fluorescence intensity measured in the visual cortex of AAV - shlc control (green symbols) and AAV - sh95 (blue symbols) injected WT animals at > P 110 plotted against ocular dominance indices. Fluorescence intensities in the visual cortex of control injected animals (AAV - shlc) or PSD-95 knockdown animals (AAV - sh95) did not correlate with their respective ocular dominance indices. 


\subsubsection{Visual acuity increase during 7 days of monocular deprivation}

In WT mice, visual acuity increases over the time course of monocular deprivation as shown in Prusky et al. (2006), where MD results in a reduction or loss of the responsiveness of cortical neurons to the closed eye and the threshold of the optomotor reflex increases in the open eye. This process depends on the structure of activity in the deprived eye (Blakemore, 1976; Bear \& Rittenhouse, 1999; Rittenhouse et al., 2006). Consequently, it was examined whether WT mice with a visual cortex restricted knockdown of PSD-95 show a similar visual acuity improvement over the 7-day time course of MD. After MD, control injected animals (AAV shlc) and PSD-95 knockdown animals were tested in the optomotor setup over the course of the MD-period daily. There was no significant difference between PSD-95 knockdown and control mice with both groups showing improved visual acuity over the time course of the 7 days MD-period. PSD-95 knockdown mice improved from $0.393 \pm 0.001 \mathrm{cyc} / \mathrm{deg}$ on day 0 to $0.502 \pm 0.002 \mathrm{cyc} /$ deg on day $7(n=7)$. WT control injected mice improved from $0.390 \pm 0.002$ cyc/deg on day 0 to $0.495 \pm 0.002 \mathrm{cyc} / \operatorname{deg}$ on day $7(n=5 ; p=0.2)$ (Figure $46 \mathrm{~A})$, corresponding to a total gain on baseline of $27.61 \pm 0.77 \%$ in PSD-95 knockdown mice and $28.61 \pm 1.15 \%$ in control mice ( $p=0.99$; $t$-test) (Figure 46 B).

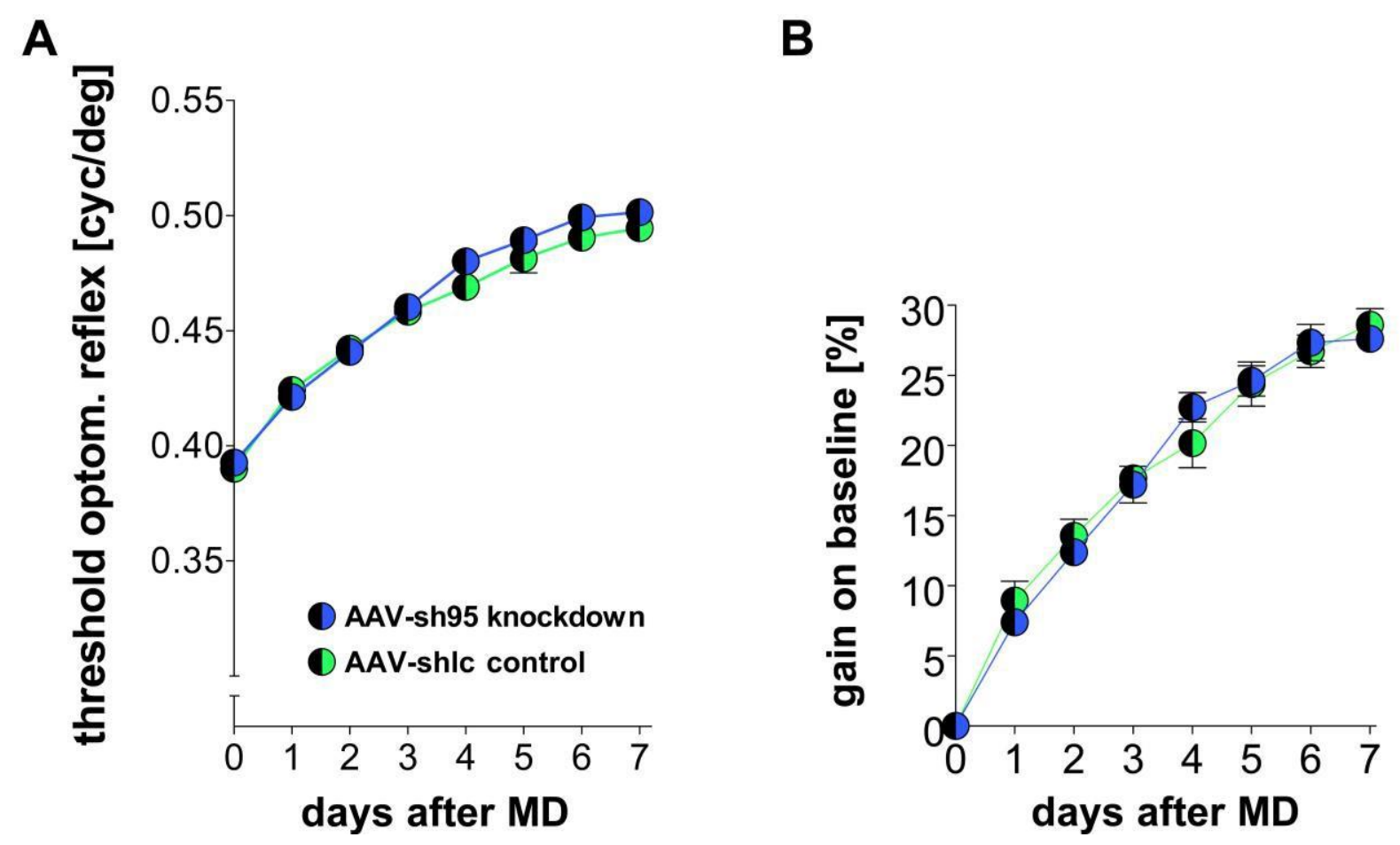

Figure 46: Time course and gain on baseline of visual acuity in WT control mice (AAV-shlc) and PSD-95 knockdown mice (AAV-sh95). Using the virtual-reality optomotor system, (A) visual acuity (maximum perceived spatial frequency) and (B) the gain on baseline over the time course of seven days of MD was determined. The 
graphs show enhancement of visual acuity after 7 days of MD in control mice (green) and PSD-95 knockdown mice (blue) with average values and error bars (SEM). The temporal course of the threshold of the optomotor reflex and the gain on baseline over the 7 days of MD was not significantly different between the two groups ( $p$ $>0.05)$.

The present data reveal that a visual cortex restricted knockdown of PSD-95 does not alter visual functions in WT mice that were measured in the visual water task (visual acuity and orientation discrimination) or in the virtual-reality optomotor system (threshold of the optomotor reflex). 


\subsection{PSD-93 KO mice during the critical period}

\subsubsection{Visual capabilities measured with the virtual-reality optomotor system}

\subsubsection{Maximum spatial frequency threshold (visual acuity)}

Since it was not known whether PSD-93 influences visual capabilities in mice, the virtualreality optomotor system (OPT; Prusky et al., 2004) was used to determine visual capabilities of PSD-93 KO and WT mice during the mid-critical period (mid CP; P 24 - 27) and late CP (P 28 - 35). The maximum spatial frequency at which the optokinetic response (OKR) of the mice was still elicited, was used to measure the visual acuity threshold of the optomotor reflex (from now referred to as 'visual acuity'). To determine general visual acuity, all mice were tested before monocular deprivation and the conditions were kept equal for all mice.

In control mice without MD, visual acuity of PSD-93 KO mice was not significantly different from age-matched WT mice and remained unchanged over the testing period during the mid CP (PSD-93 KO: $0.384 \pm 0.001 \mathrm{cyc} / \mathrm{deg} ; \mathrm{n}=5 ; \mathrm{WT}: 0.390 \pm 0.001 \mathrm{cyc} / \mathrm{deg} ; \mathrm{n}=3 ; \mathrm{p}>0.05$; unpaired t test) and during the late CP (PSD-93 KO: $0.385 \pm 0.001 \mathrm{cyc} / \mathrm{deg} ; n=5$; WT: $0.392 \pm$ $0.00 \mathrm{cyc} / \mathrm{deg} ; \mathrm{n}=5 ; \mathrm{p}>0.05$; unpaired $\mathrm{t}$ test). The data indicate that visual acuity of PSD-93 KO mice is not compromised and similar to that of WT mice (Figure 48).

A

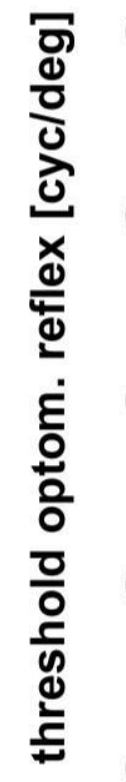

B

late critical period: P28-35

WT

Figure 48: Visual acuity of PSD-93 KO and WT mice during mid and late critical period measured with the virtual-reality optomotor system (Prusky et al., 2004). The maximum spatial frequency eliciting the optokinetic response of a mouse is defined as visual acuity. (A) Visual acuity of PSD-93 KO and WT mice during mid CP (P 24- 
27) and (B) during late CP (P 28-35) was similar in both genotypes and ages ( $p>0.05$ for all comparisons, t-test). Data represent mean \pm SEM.

\subsubsection{Visual acuity increase during four days of monocular deprivation}

It was examined whether WT and PSD-93 KO mice show a similar visual acuity improvement over the 4 days time course of MD both during mid and late CP.

During the mid CP (P 24 - 27), there was no significant difference between PSD-93 KO and WT mice with both groups showing improved visual acuity over the time course of the 4-day MDperiod with PSD-93 KO improving from $0.383 \pm 0.005 \mathrm{cyc} / \mathrm{deg}$ on day 0 to $0.464 \pm 0.008$ cyc/deg on day $4(n=6)$. WT mice improved from $0.374 \pm 0.003 \mathrm{cyc} /$ deg on day 0 to $0.459 \pm$ $0.006 \mathrm{cyc} / \mathrm{deg}$ on day $4(n=4)(p>0.05$, repeated measure ANOVA with Bonferroni correction) (Figure 49 A), corresponding to a total gain on baseline of $23 \pm 1.3 \%$ in WT and 22 $\pm 2.7 \%$ in PSD-93 KO mice ( $p=0.86$; t-test) (Figure $50 \mathrm{~A}$ ).
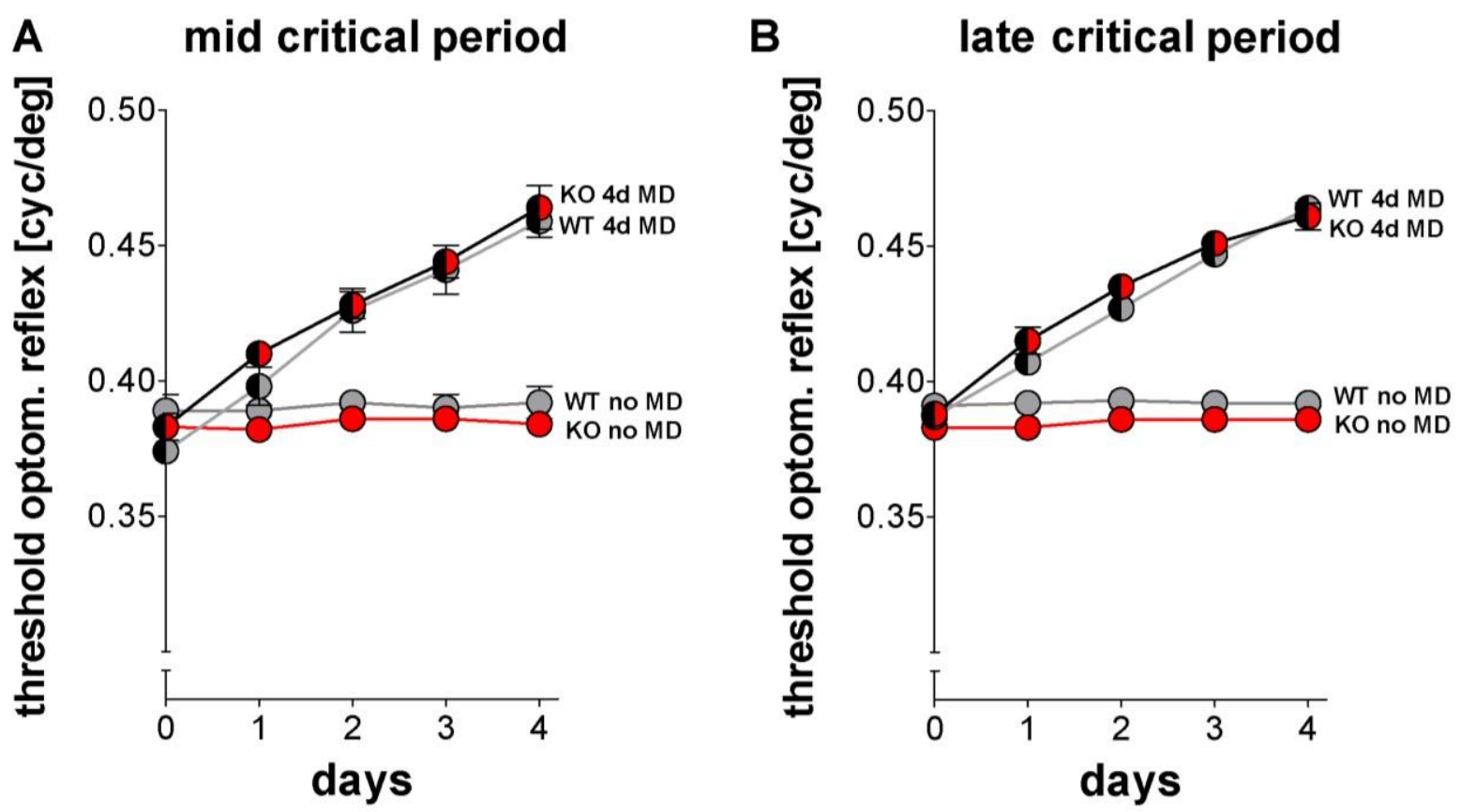

Figure 49: Time course of visual acuity during (A) the mid and (B) late critical period of WT and PSD-93 KO mice. Using the virtual-reality optomotor system, visual acuity (maximum perceived spatial frequency) of PSD$93 \mathrm{KO}$ and WT mice was determined. The graphs show the enhancement of visual acuity after 4 days of MD (halffilled circles) in WT (gray) and PSD-93 KO (red) mice and of animals without MD (filled circles). The average values of visual acuity of all animals in one group are presented in cycles/degree on the $y$-axis with error bars (SEM). 
During the late CP (P 28-35), there was no significant difference between PSD-93 KO and WT mice with both groups showing similar visual acuity improvement after 4 days of MD. PSD-93 KO mice improved from $0.388 \pm 0.004 \mathrm{cyc} / \mathrm{deg}$ on day 0 to $0.461 \pm 0.004 \mathrm{cyc} / \mathrm{deg}$ on day 4 ( $\mathrm{n}=$ 6). WT mice improved from $0.387 \pm 0.004 \mathrm{cyc} / \mathrm{deg}$ on day 0 to $0.464 \pm 0.003 \mathrm{cyc} / \mathrm{deg}$ on day 4 ( $n=7 ; p>0.05$, repeated measure ANOVA with Bonferroni correction) (Figure 49 B), corresponding to a total gain on baseline of $20 \pm 1.4 \%$ in WT and $19 \pm 1.4 \%$ in PSD-93 KO mice $(p=0.62$; t-test) (Figure $50 \mathrm{~A}, \mathrm{~B})$.
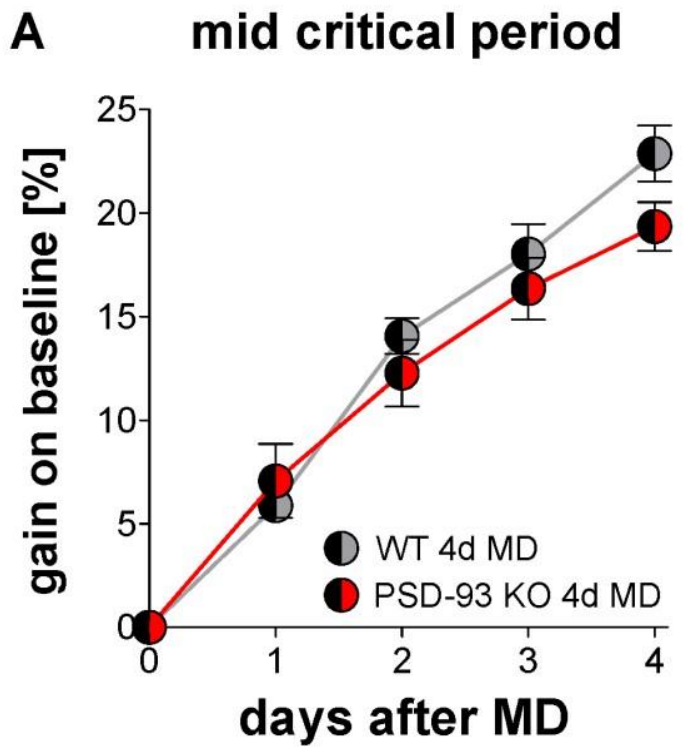

\section{B late critical period}

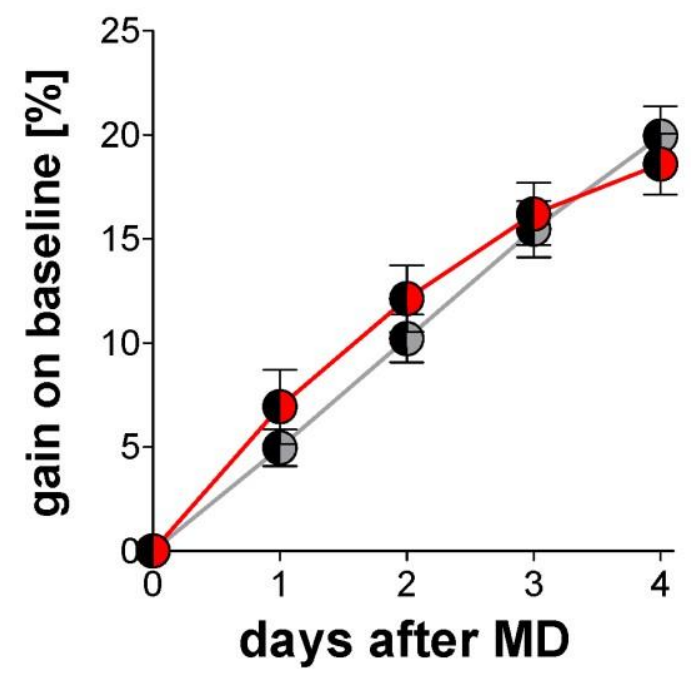

Figure 50: Gain on baseline of visual acuity of WT and PSD-93 KO mice after 4 days of MD during the mid and late critical period measured with the virtual optomotor system (Prusky, 2004). The graph shows the gain on baseline of visual acuity in WT and PSD-93 KO mice over 4 days of MD. The average gain on baseline of all animals in one group is presented in per cent on the y-axis with respective error bars (standard deviation). Both groups did not differ from each other in the gain on baseline (t-test, $p>0.05$, for all comparisons).

\subsection{Minimum contrast threshold (contrast sensitivity)}

\subsubsection{Contrast sensitivity of WT and PSD-93 KO mice during the mid CP}

In addition to the maximum spatial frequency threshold of the optomotor reflex, the minimum contrast threshold at which the OKR can be elicited, was also determined using the optomotor system. This threshold is a measure of contrast sensitivity and was determined at six different spatial frequencies: $0.031,0.064,0.092,0.103,0.192$, and $0.272 \mathrm{cyc} / \mathrm{deg}$ before and after 4 days of $M D$, respectively. At each of the spatial frequencies, the contrast was 
decreased stepwise until the OKR could not be observed anymore. Contrast sensitivity was determined for both PSD-93 KO and WT mice during the mid and late CP in control groups without MD and in mice after 4 days of MD.

WT mice during the mid CP showed a contrast sensitivity of $4 \pm 0.06$ at a spatial frequency of $0.031 \mathrm{cyc} / \mathrm{deg}, 12.8 \pm 0.53$ at $0.064 \mathrm{cyc} / \mathrm{deg}, 11.7 \pm 0.42$ at $0.092 \mathrm{cyc} / \mathrm{deg}, 11.5 \pm 0.39$ at 0.103 cyc/deg, $8.3 \pm 0.12$ at $0.192 \mathrm{cyc} / \mathrm{deg}$ and $3 \pm 0.04$ at $0.272 \mathrm{cyc} / \mathrm{deg}$. The semi log plot (Figure $51 \mathrm{~A}$ ) is showing a sickle shaped curve which was already described for C57BI/6J WT mice from Prusky et al. (2004).

PSD-93 KO mice during the mid CP showed contrast sensitivities at a spatial frequency of $0.031 \mathrm{cyc} / \mathrm{deg}, 4 \pm 0.03$, at $0.064 \mathrm{cyc} / \mathrm{deg} 12.8 \pm 0.17$, at $0.092 \mathrm{cyc} / \mathrm{deg} 11.6 \pm 0.13$, at 0.103 cyc/deg $11.2 \pm 0.15$, at $0.192 \mathrm{cyc} / \mathrm{deg} 8.0 \pm 0.09$ and at $0.272 \mathrm{cyc} / \mathrm{deg} 3.9 \pm 0.03$ as shown in Figure $51 \mathrm{~B}$.

A

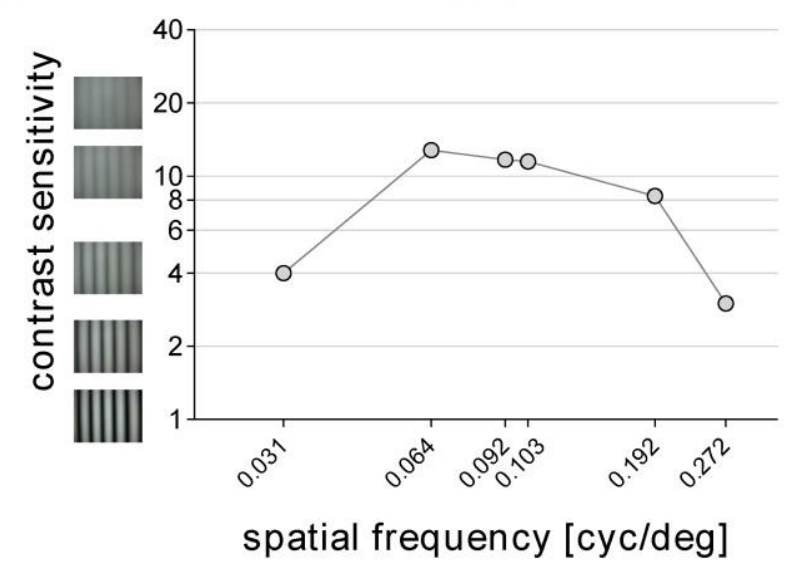

B

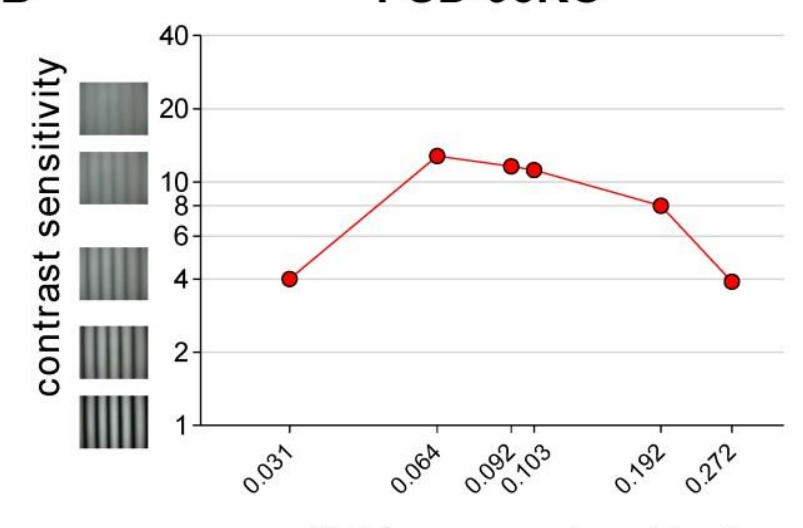

spatial frequency [cyc/deg]

Figure 51: Contrast sensitivity of (A) WT and (B) PSD-93 KO mice during the mid critical period measured with the virtual - reality optomotor system (Prusky et al., 2004). Contrast sensitivity is plotted as a function of six different spatial frequencies in cycles per degree (cyc/deg). Contrast sensitivity was similar in WT (gray) and PSD$93 \mathrm{KO}$ mice (red) ( $\mathrm{p}=0.47$; ANOVA) and maximal contrast sensitivity was $12.8 \pm 0.53$ in WT compared to $12.8 \pm$ 0.17 in PSD-93 KO mice at the spatial frequency of $0.064 \mathrm{cyc} / \mathrm{deg}$.

Hence, contrast sensitivities of PSD-93 KO and WT mice were similar for all measured six different spatial frequencies (mid CP: $p=0.88$; ANOVA), demonstrating that PSD-93 KO mice are not impaired in the optokinetic reflex for contrast sensitivity. In both genotypes, contrast sensitivities peaked at $0.064 \mathrm{cyc} / \mathrm{deg}$ as previously described by Prusky et al. (2004), and were lowest at 0.031 and $0.272 \mathrm{cyc} / \mathrm{deg}$, respectively. 
WT and PSD-93 KO mice during the mid CP were also tested after 4 days of MD to investigate their interocular plasticity of vision. Therefore, contrast sensitivity of the non-deprived eye was measured with the virtual-reality optomotor system after MD daily. As in the control groups without MD, contrast sensitivities were measured at six different spatial frequencies. On each day following monocular deprivation, the contrast sensitivity of all measured spatial frequencies increased substantially.

Contrast sensitivity in WT mice after 4 days of MD increased from day 0 to day 4 from $3.95 \pm$ 0.01 to $4.5 \pm 0.13$ at a spatial frequency of $0.031 \mathrm{cyc} / \mathrm{deg}$, from $12.65 \pm 0.88$ to $17.9 \pm 0.55$ at $0.064 \mathrm{cyc} / \mathrm{deg}$, from $11.58 \pm 0.74$ to $16.36 \pm 0.52$ at $0.092 \mathrm{cyc} / \mathrm{deg}$, from $11.3 \pm 0.71$ to $16 \pm$ 0.56 at $0.103 \mathrm{cyc} / \mathrm{deg}$, from $8.2 \pm 0.1$ to $10.54 \pm 0.9$ at $0.192 \mathrm{cyc} / \mathrm{deg}$, from $3.85 \pm 0.07$ to 4.4 \pm 0.07 at $0.272 \mathrm{cyc} / \mathrm{deg}$ with a peak of contrast sensitivity at $0.064 \mathrm{cyc} / \mathrm{deg}$ (Figure $52 \mathrm{~A}$ ). Likewise, contrast sensitivity of PSD-93 KO mice after 4 days of MD increased from day 0 to day 4 from $4.01 \pm 0.02$ to $4.5 \pm 0.08$ at a spatial frequency of 0.031 cyc $/ \mathrm{deg}$, from $13.03 \pm 0.3$ to $17.7 \pm 0.83$ at $0.064 \mathrm{cyc} / \mathrm{deg}$, from $11.9 \pm 0.39$ to $16.53 \pm 0.83$ at $0.092 \mathrm{cyc} / \mathrm{deg}$, from 11.5 \pm 0.4 to $16.03 \pm 0.77$ at $0.103 \mathrm{cyc} / \mathrm{deg}$, from $8.1 \pm 0.2$ to $10.34 \pm 0.45$ at $0.192 \mathrm{cyc} / \mathrm{deg}$, from $3.9 \pm 0.05$ to $4.8 \pm 0.59$ at $0.272 \mathrm{cyc} / \mathrm{deg}$ (Figure $52 \mathrm{~B}$ ). The genotype did not influence contrast sensitivity ( $p=0.47$; ANOVA) or the improvement of it after four days of $M D(p=0.55$; ANOVA).

A

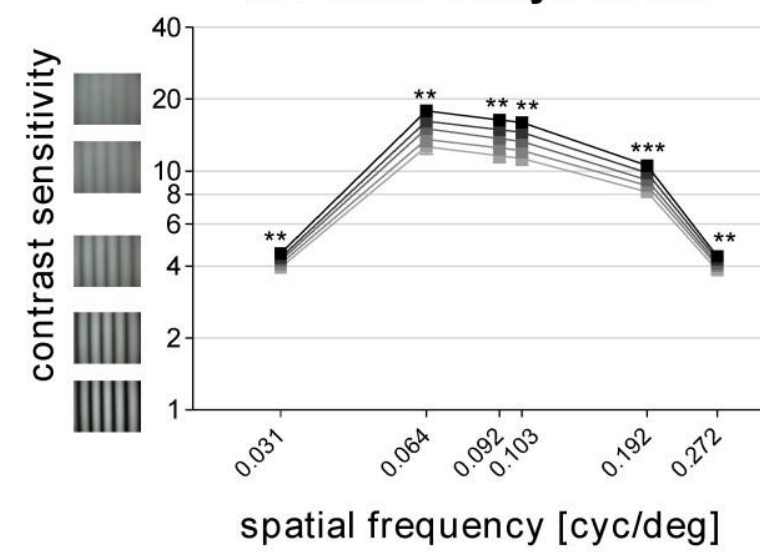

B PSD-93 KO after 4 days of MD

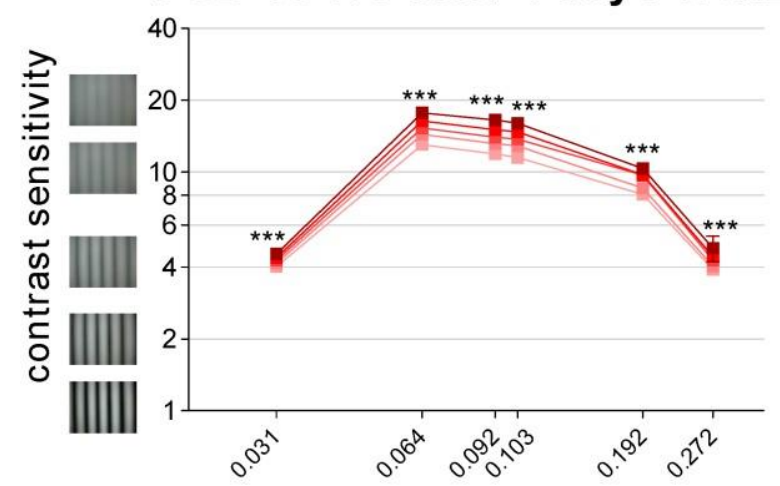

spatial frequency [cyc/deg]

Figure 52: Enhancement of contrast sensitivity of (A) WT and (B) PSD-93 KO mice during the mid-critical period after monocular deprivation measured with the virtual-reality optomotor system (Prusky et al., 2004). Contrast sensitivity is plotted as a function of six different spatial frequencies in cycles per degree (cyc/deg). Both WT (gray) and PSD-93 KO mice (red) showed a significant improvement of contrast sensitivity during the time course of $M D(p<0.01$; t-test). Genotype did not have an influence on the improvement of contrast sensitivity after MD ( $p=0.55$; ANOVA). 


\subsubsection{Contrast sensitivity of WT and PSD-93 KO mice during the late CP}

Contrast sensitivity was determined for both PSD-93 KO and WT mice during the late CP in control groups without MD and in mice after 4 days of MD, respectively.

WT mice showed contrast sensitivity of $4.1 \pm 0.05$ at a spatial frequency of $0.031 \mathrm{cyc} / \mathrm{deg}, 13$ \pm 0.22 at $0.064 \mathrm{cyc} / \mathrm{deg}, 12 \pm 0.25$ at $0.092 \mathrm{cyc} / \mathrm{deg}, 11.7 \pm 0.25$ at $0.103 \mathrm{cyc} / \mathrm{deg}, 8.4 \pm 0.2$ at $0.192 \mathrm{cyc} / \mathrm{deg}$ and $4 \pm 0.05$ at $0.272 \mathrm{cyc} / \mathrm{deg}$. The semi log plot (Figure $53 \mathrm{~A}$ ) is showing a sickle shaped curve which was already described for C57BI/6J WT mice from Prusky et al. (2004) and similar to that of WT mice during the mid CP ( $p>0.05$; ANOVA).

PSD-93 KO mice showed contrast sensitivity of $4 \pm 0.01$ at a spatial frequency of $0.031 \mathrm{cyc} / \mathrm{deg}$, $13 \pm 0.13$ at $0.064 \mathrm{cyc} / \mathrm{deg}, 11.9 \pm 0.1$ at $0.092 \mathrm{cyc} / \mathrm{deg}, 11.6 \pm 0.12$ at $0.103 \mathrm{cyc} / \mathrm{deg}, 8.1 \pm 0.1$ at $0.192 \mathrm{cyc} / \mathrm{deg}$ and $4 \pm 0.06$ at $0.272 \mathrm{cyc} / \mathrm{deg}$ as shown in Figure $53 \mathrm{~B}$.

A

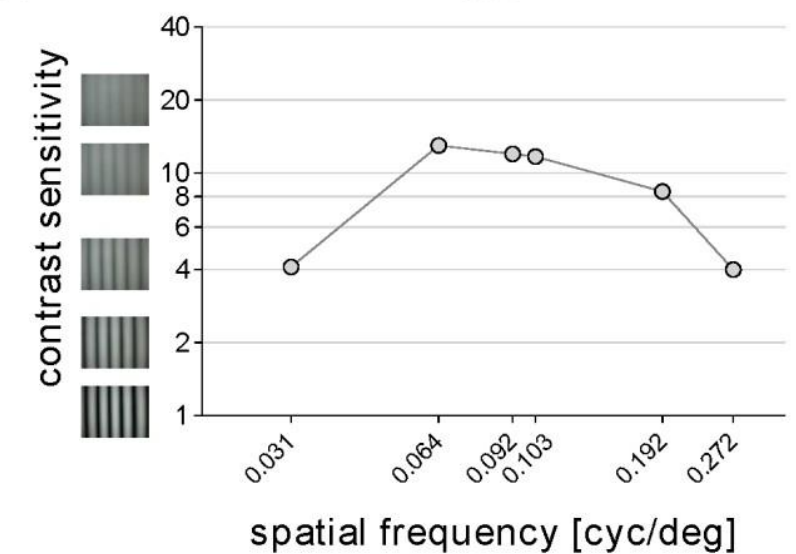

B
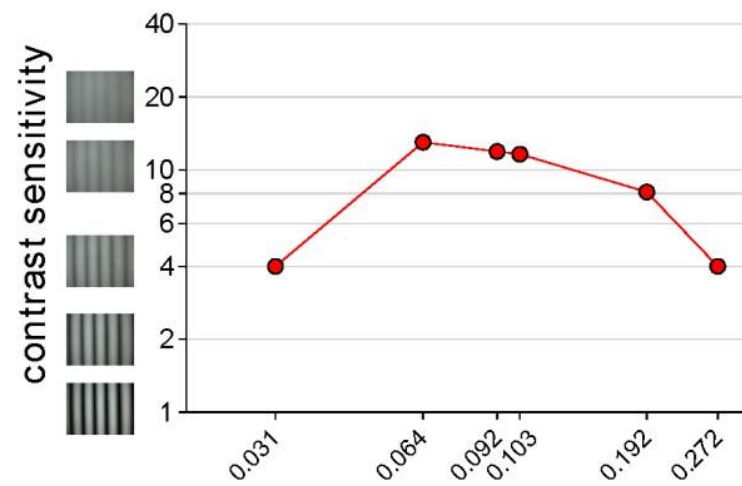

spatial frequency [cyc/deg]

Figure 53: Contrast sensitivity of (A) WT and (B) PSD-93 KO mice during the late critical period measured with the virtual-reality optomotor system (Prusky et al., 2004). Contrast sensitivity is plotted as a function of six different spatial frequencies in cycles per degree (cyc/deg). Contrast sensitivity was similar in WT (gray) and PSD$93 \mathrm{KO}$ mice (red) ( $=0.4 ;$ ANOVA) and maximal contrast sensitivity was $13 \pm 0.22$ in WT compared to $13 \pm 0.13$ in PSD-93 KO mice at the spatial frequency of $0.064 \mathrm{cyc} / \mathrm{deg}$.

Hence, contrast sensitivities of PSD-93 KO and WT mice were similar in all six different spatial frequencies ( $p=0.4$; ANOVA), demonstrating that PSD-93 KO mice are not impaired in the optokinetic reflex for contrast sensitivity. In both genotypes, contrast sensitivities peaked at 0.064 cyc/deg as previously described by Prusky et al. (2004) and were lowest at 0.031 and $0.272 \mathrm{cyc} / \mathrm{deg}$, respectively. 
Additionally, WT and PSD-93 KO mice during the late CP were also tested after 4 days of MD to investigate their interocular plasticity of vision. Therefore, contrast sensitivity of the nondeprived eye was measured from day zero to day four daily. As in the control groups without $\mathrm{MD}$, contrast sensitivities were measured at all six different spatial frequencies. On each day following monocular deprivation, the contrast sensitivity of all measured spatial frequencies increased substantially.

Contrast sensitivity in WT mice after 4 days of MD increased from day 0 to day 4 from $4.1 \pm$ 0.04 to $4.8 \pm 0.1$ at a spatial frequency of $0.031 \mathrm{cyc} / \mathrm{deg}$, from $13.4 \pm 0.27$ to $19.3 \pm 0.62$ at $0.064 \mathrm{cyc} / \mathrm{deg}$, from $12.6 \pm 0.33$ to $19.2 \pm 0.61$ at $0.092 \mathrm{cyc} / \mathrm{deg}$, from $12.2 \pm 0.31$ to $16.7 \pm$ 0.46 at $0.103 \mathrm{cyc} / \mathrm{deg}$, from $8.6 \pm 0.2$ to $11.2 \pm 0.4$ at $0.192 \mathrm{cyc} / \mathrm{deg}$, from $4.03 \pm 0.04$ to $4.7 \pm$ 0.01 at $0.272 \mathrm{cyc} / \mathrm{deg}$ with a peak of contrast sensitivity at $0.064 \mathrm{cyc} / \mathrm{deg}$ (Figure $54 \mathrm{~A}$ ). Likewise, contrast sensitivity of PSD-93 KO mice after 4 days of MD increased from day 0 to day 4 from $4.1 \pm 0.06$ to $5.1 \pm 0.22$ at a spatial frequency of $0.031 \mathrm{cyc} / \mathrm{deg}$, from $13.2 \pm 0.47$ to $18.6 \pm 0.94$ at $0.064 \mathrm{cyc} / \mathrm{deg}$, from $12.3 \pm 0.59$ to $17.4 \pm 1.2$ at $0.092 \mathrm{cyc} / \mathrm{deg}$, from $12.1 \pm$ 0.59 to $16.7 \pm 1.1$ at $0.103 \mathrm{cyc} / \mathrm{deg}$, from $8.2 \pm 0.26$ to $11.5 \pm 0.85$ at $0.192 \mathrm{cyc} / \mathrm{deg}$, from 4.1 \pm 0.09 to $5.02 \pm 0.23$ at $0.272 \mathrm{cyc} / \mathrm{deg}$ (Figure $54 \mathrm{~B}$ ). The genotype did not have an effect on contrast sensitivity $(p=0.4$; ANOVA), or the improvement of it after four days of $M D(p=0.47$; ANOVA).

A

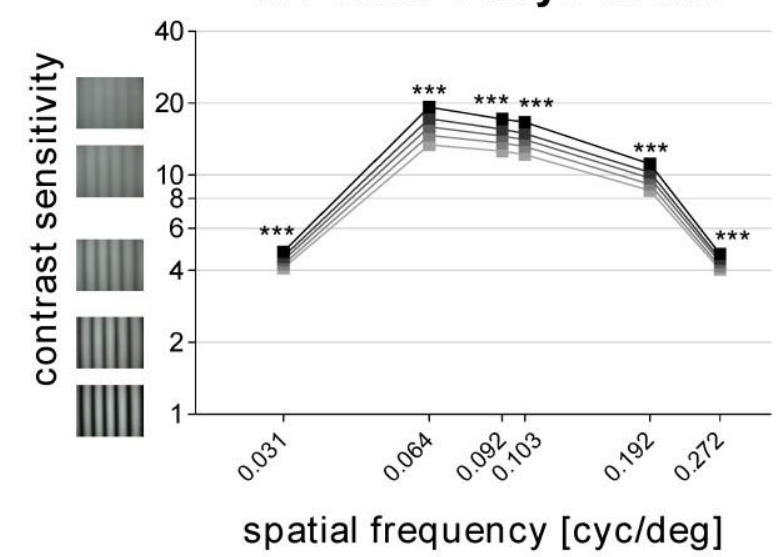

B PSD-93 KO after 4 days of MD

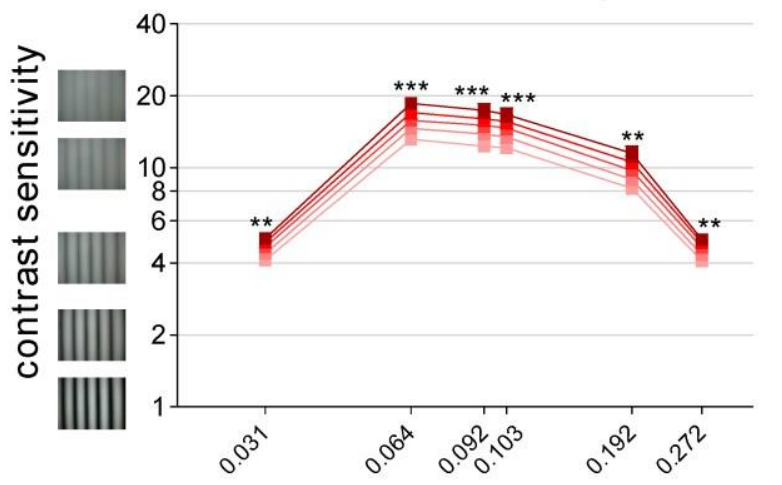

spatial frequency [cyc/deg]

Figure 54: Enhancement of contrast sensitivity of (A) WT and (B) PSD-93 KO mice during the late critical period after 4 days of monocular deprivation measured with the virtual-reality optomotor system (Prusky et al., 2004). Contrast sensitivity is plotted as a function of six different spatial frequencies in cycles per degree (cyc/deg). Both WT (gray) and PSD-93 KO (red) mice showed a significant improvement of contrast sensitivity after 4 days of $M D(p<0.01$; t-test) and genotype did not have an influence on the improvement of contrast sensitivity after MD ( $p=0.47$; ANOVA). 
Testing visual capabilities of PSD-93 KO mice in the optomotor setup revealed no visual deficits. PSD-93 KO mice were as good as WT mice in both the maximum spatial frequency threshold of the optomotor reflex (referred to as visual acuity) and contrast sensitivity. Since measurements in the optomotor setup rely on subcortical processes, visual acuity and orientation discrimination of PSD-93 KO and WT mice was additionally tested in the visual water task (VWT). Using this method allows to get better insights if a loss of PSD-93 might influence visual capabilities.

\subsection{Visual capabilities of PSD-93 KO and WT mice measured in the VWT}

To get better insights if a loss of PSD-93 might influence visual capabilities, visual acuity and orientation discrimination of PSD-93 KO and WT mice (P 60) was tested in the visual water task, a visual discrimination task based on reinforcement learning (Prusky et al., 2000).

WT mice $(n=8)$ showed normal visual acuity $(0.56 \pm 0.04 \mathrm{cyc} / \mathrm{deg})$ which was comparable to that of WT mice tested before (see: Figure 38 A; $p>0.05$, t-test). PSD-93 KO mice $(n=11)$ showed visual acuity of $0.57 \pm 0.05 \mathrm{cyc} / \mathrm{deg}$ which was similar to that of WT mice $(p=0.94, t-$ test) (Figure 55 B).

In contrast, testing orientation discrimination in these mice revealed that PSD-93 KO mice are severely impaired in orientation discrimination $\left(34 \pm 1.9^{\circ}\right)$ in comparison to WT mice $(12.4 \pm$ $2.7^{\circ} ; \mathrm{p}<0.001$, t-test) (Figure $55 \mathrm{C}$ ). 
A

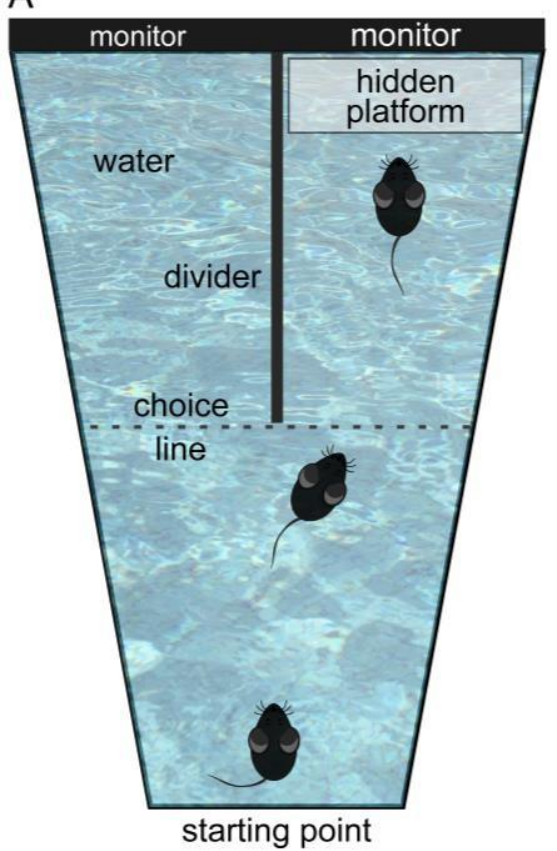

B
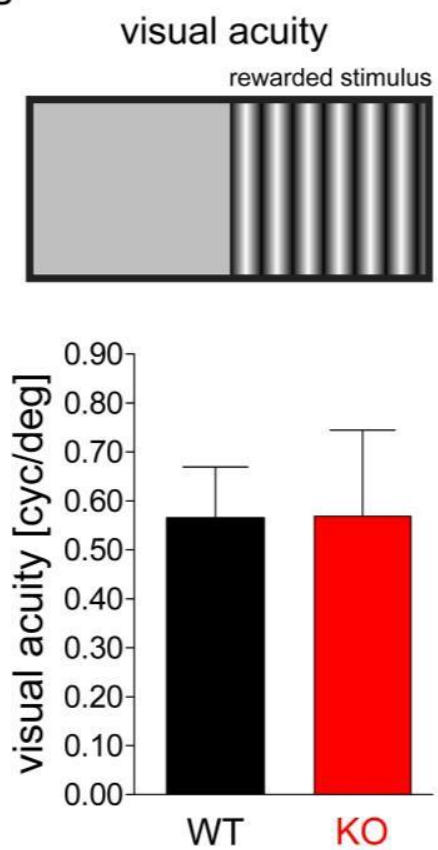

C
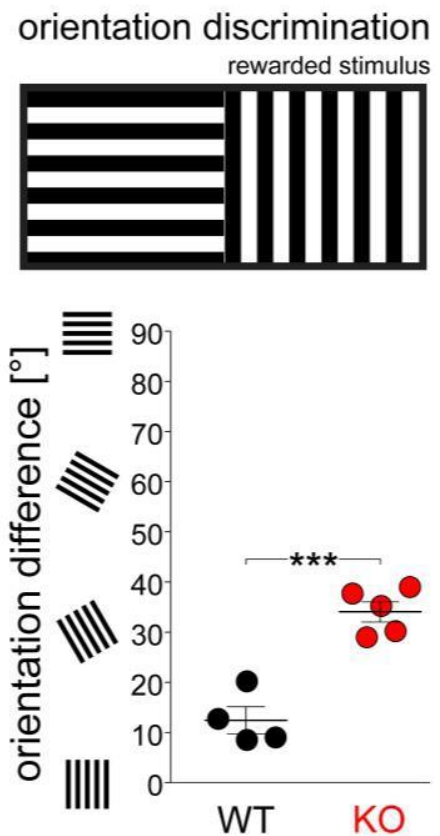

Figure 55: Maximum spatial frequency threshold and minimum angle difference of PSD-93 KO and WT mice determined in the visual water task (Prusky, 2000). (A) Schematic overview of the visual water task which allows testing of both visual acuity and orientation discrimination of mice. (B) To determine visual acuity, mice are trained to swim towards a monitor screen displaying a stationary, sinusoidal grating of black and white bars, while isoluminent gray is displayed on the reference screen. Visual acuity of PSD-93 KO mice was similar to that of WT control mice ( $p=0.94$, t-test). (C) To test orientation discrimination, mice are trained to distinguish a horizontal grating from a vertical grating. In front of the stimulus screen, a hidden platform is placed underneath the water surface serving as a reward. Orientation discrimination was significantly impaired in PSD-93 KO mice compared to WT control mice ( $p<0.001$, t-test). Each data point indicates the mean of 3 values obtained from each animal. Bars represent mean \pm SEM.

\subsection{OD-plasticity of WT and PSD-93 KO mice during mid critical period}

One very interesting question in this thesis was if PSD-93 KO mice show cortical plasticity. PSD-

93 is an important anchor protein in the PSD (McGee et al., 2006). A lack of PSD-93 leads to enhanced LTD and diminished LTP. Furthermore, PSD-93 KO mice lack AMPA receptor silent synapses in the visual cortex already during the critical period (Favaro, doctoral thesis, 2014; Schlüter laboratory, ENI, Göttingen), which was exactly the opposite finding to that of high AMPA receptor silent synapse numbers in PSD-95 KO mice throughout life (Huang et al., 2015) (Figure 56). These AMPA receptor silent synapses are very important for OD-plasticity (Huang et al., 2015) and prevent the closure of the critical period for OD-plasticity in PSD-95 KO mice (see: chapter 3.1.). 


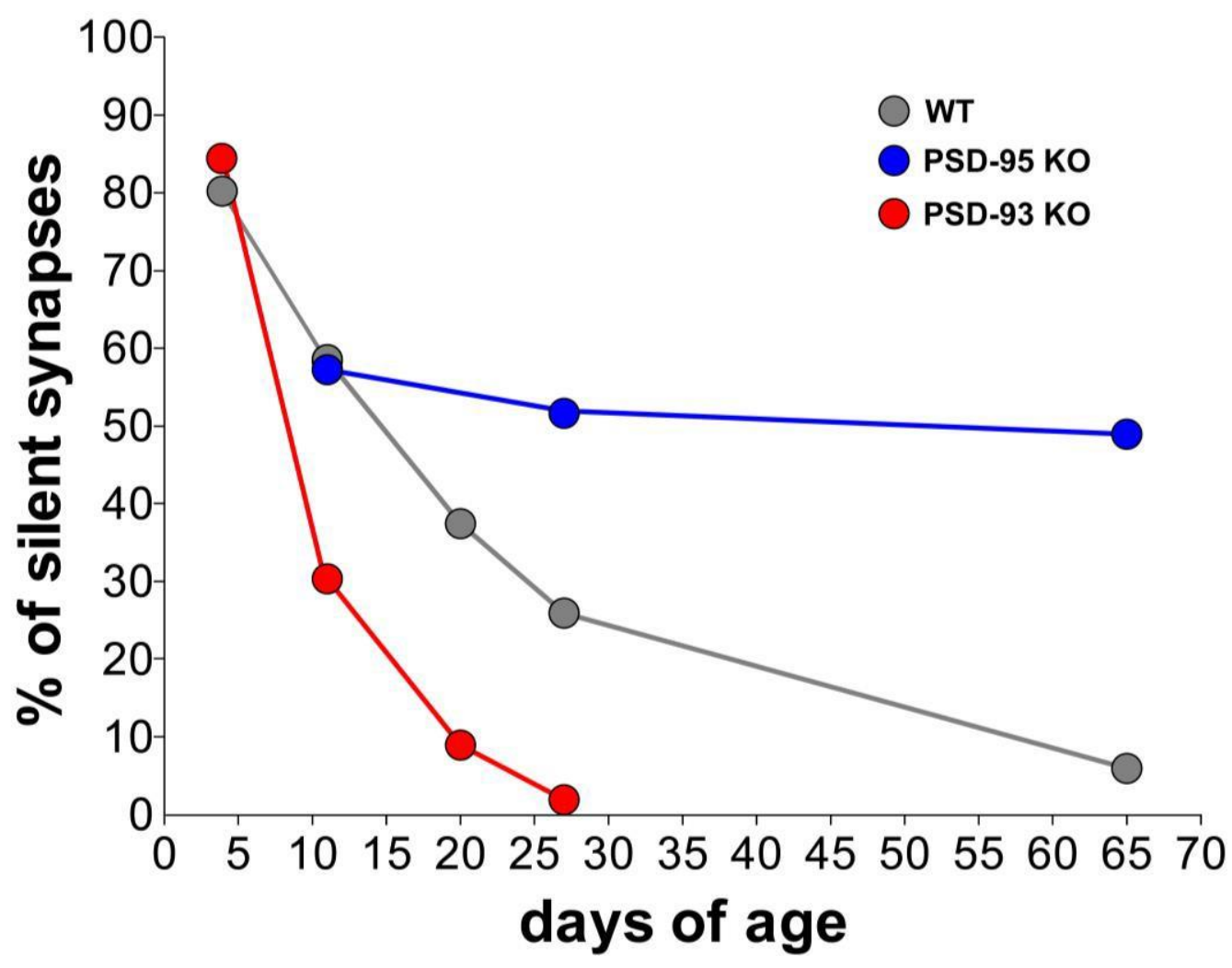

Figure 56: Number of AMPA receptor silent synapses in percent (\%) measured in the VC of WT, PSD-95 KO and PSD-93 KO mice. The number of AMPA receptor silent synapses is gradually decreasing in WT mice (gray) from 80 $\pm 0.05 \%$ (P $3-P 5)$ to $58.6 \pm 0.04 \%$ (P $10-P 12$ ) and further to $37.5 \pm 0.06 \%$ (P 19-P21). During the critical period, the numbers of AMPA silent synapses reach $26 \pm 0.05 \%$ (P $25-$ P 30) which further decrease even below $9 \%$ after the critical period. In contrast, the numbers of AMPA receptor silent synapses remain high in PSD-95 KO mice (blue) throughout life about 55 \%. In PSD-93 KO mice, AMPA receptor silent synapses were abundant before eye opening at P $3-$ P 5 (85 $\pm 0.04 \%)$ but already declined to $30 \pm 0.06 \%$ between P $10-P 12$. Interestingly, already at the very beginning of the critical period (P 20), AMPA receptor silent synapses dropped to $9 \pm 0.05 \%$ in PSD-93 KO mice and were nearly absent at P 25 ( $2 \pm 0.06 \%$ ). Data obtained by Huang (doctoral thesis, 2013; Huang et al., 2015) and Favaro (doctoral thesis, 2014).

PSD-95 and PSD-93 KO mice show exactly the opposite phenotype regarding the maturation of AMPA receptor silent synapses in the visual cortex (Figure 56), with heightened numbers in PSD-95 KO mice during and after the critical period and a rapid loss of them in PSD-93 KO already during the critical period. Since PSD-95 KO mice show OD-plasticity lifelong, it was of high interest to investigate whether OD-plasticity would be absent in PSD-93 KO mice. Therefore, visual cortical responses of PSD-93 KO and WT mice with and without 4 days of MD during mid and late $\mathrm{CP}$ were recorded using optical imaging of intrinsic signals in vivo developed by Kalatsky and Stryker (2003) and Cang et al. (2005a). Visual cortical response 
amplitudes in the binocular part of V1 after stimulation of either the contra- or the ipsilateral eye were compared, respectively. In this experiment, visual stimuli consisted of a horizontal bar $\left(2^{\circ}\right.$ wide, $20^{\circ}$ long) moving in two different directions $\left(90^{\circ}\right.$ and $\left.270^{\circ}\right)$ in the visual field of the mouse. The ocular dominance index (ODI) was calculated to quantify the shift of ocular dominance in V1. It serves as a measure for OD-plasticity and is defined as $(C-I) /(C+I)$ with $\mathrm{C}$ and I referring to V1 binocular region activity induced by the contra- or the ipsilateral eye. A regular WT control mouse without MD usually shows dominance of the contralateral eye and exhibits a positive ODI (Cang et al., 2005 a). In critical period standard cage raised mice, 4 days of MD drive the neurons in favor of the open (ipsilateral) eye and the ODI is negative. The same holds true for young adult WT mice (<P 110) after 7 days of MD. The bias of neurons in the binocular zone shifts to stronger inputs from the ipsilateral eye. Until now, it was not known whether PSD-93 KO mice would exhibit OD-plasticity after monocular deprivation. Optical imaging of intrinsic signals in PSD-93 KO mice was performed after 4 days of MD between P 28 - 35 and after 7 days of MD between P 50 - 80 (experiments done by Götze, Löwel laboratory, Göttingen). In both age groups, ocular dominance indices varied strongly (ODIs between 0.01-0.31), which did not allow a conclusion about the function of PSD-93 in OD-plasticity of mice.

Therefore, in the present PhD thesis, OD-plasticity was investigated in PSD-93 KO mice during the CP (P 24 - 35) using optical imaging of intrinsic signals after 4 days of MD (26 out of 53 experiments were performed by Hosang (Master thesis 2014)).

The critical period of mice starts around P 20 and closes around P 35 (Levelt \& Hübener, 2012). To allow a thorough examination of OD-plasticity during the time course of the critical period, the $\mathrm{CP}$ was subdivided into two parts: the mid $\mathrm{CP}$ ranging from $\mathrm{P} 24-\mathrm{P} 27$ and the late $\mathrm{CP}$ ranging from P 28 - 35. Since mice cannot be weaned before P 20, this age was the first possible point of time to monocularly deprive the animals. Consequently, P 24 was the first possible point of time where OD-plasticity could be tested.

In WT and PSD-93 KO mice without MD (control groups), visual stimulation of the contralateral eye (contra) eye induced stronger visual cortical activation than after visual stimulation of the ipsilateral (ipsi) eye. Activity patches obtained from the V1 after stimulation of the contra eye were always darker than those after ipsi eye stimulation. This phenomenon 
indicates the typical contralateral dominance in the binocular region of V1 in mice (Figure 57). Representative 2-dimensional ocular dominance maps in the left visual V1 of both genotypes showed warm, red colors indicating contralateral dominance (Figure 57 A - D), and all control mice exhibited positive ODI.

WT no MD

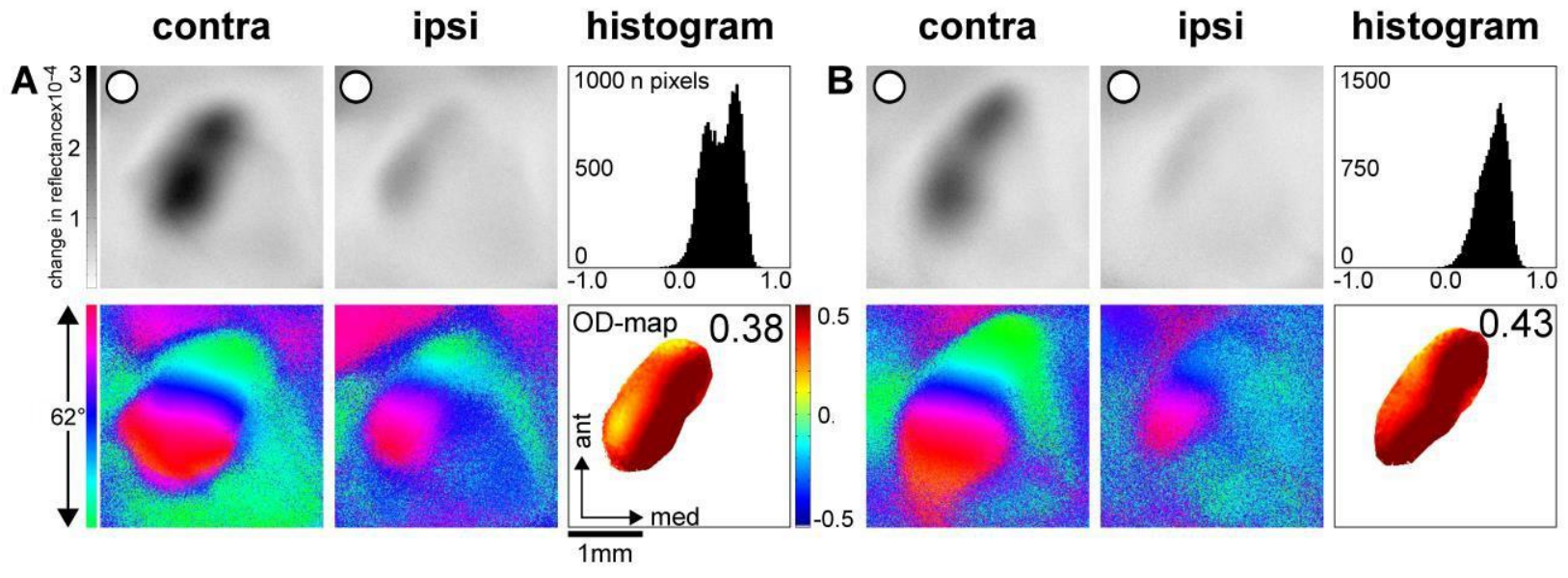

PSD-93 KO no MD
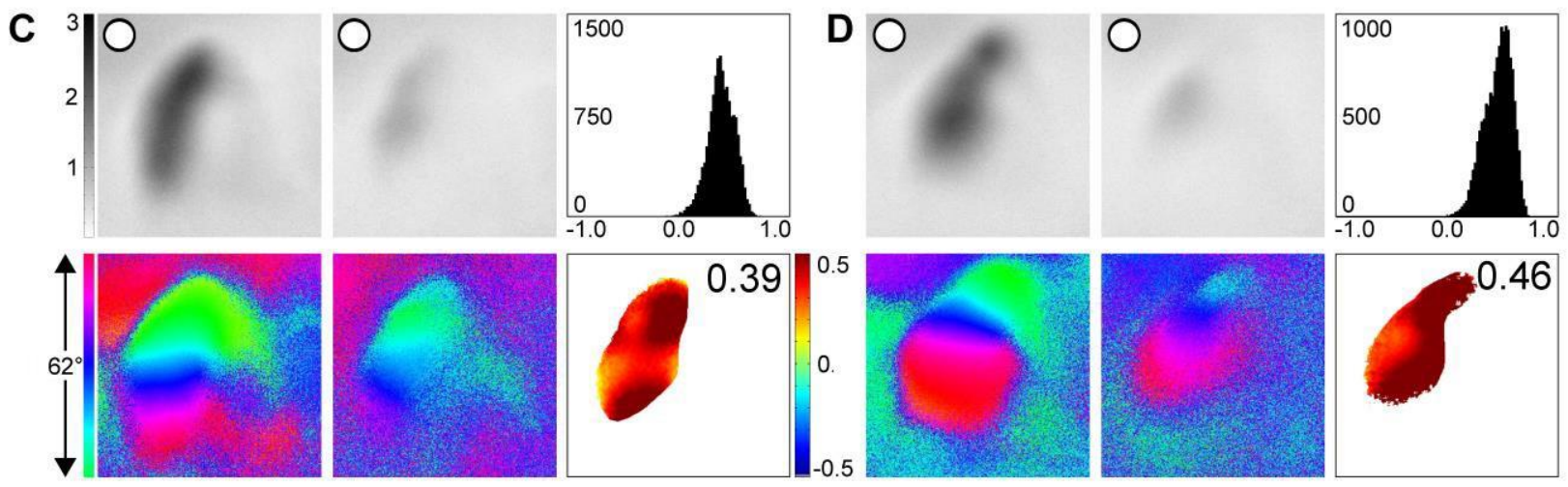

Figure 57: Representative examples of ocular dominance maps and indices in WT and PSD-93 KO mice without MD obtained with optical imaging of intrinsic signals in vivo. Optical imaging maps of contralateral and ipsilateral cortical responses to visual stimulation of either eye with a horizontal moving bar (elevation) of $20^{\circ}$ are displayed from (A, B) two WT mice and (C, D) two PSD-93 KO mice without MD, respectively. Grayscale coded response magnitude maps (top rows, expressed as fractional change in reflectance $\times 10^{-4}$ ), color-coded phase maps of retinotopy (bottom rows), histograms of the OD-scores (top right of panels) and color-coded OD-maps (right bottom including the average ODI value), are illustrated. In control mice of both genotypes, the activity patches evoked by stimulation on the contralateral (contra) eye were always darker than those evoked by ipsilateral (ipsi) eye stimulation. The average ODI was always positive and warm, red colors prevailed in the 2dimensional OD-map, indicating contralateral dominance. 
After 4 days of monocular deprivation, in both WT and PSD-93 KO mice, cortical activation was weakened after visual stimulation of the contra eye, whereas ipsi eye stimulation activated the cortex stronger, hence showing OD-plasticity. The representative 2-dimensional ocular dominance maps in the left V1 of both genotypes showed cold, blue colors indicating ipsilateral dominance (Figure 58), the histograms were shifted to the left and all mice exhibited negative ODI.

\section{WT after 4 days of MD}

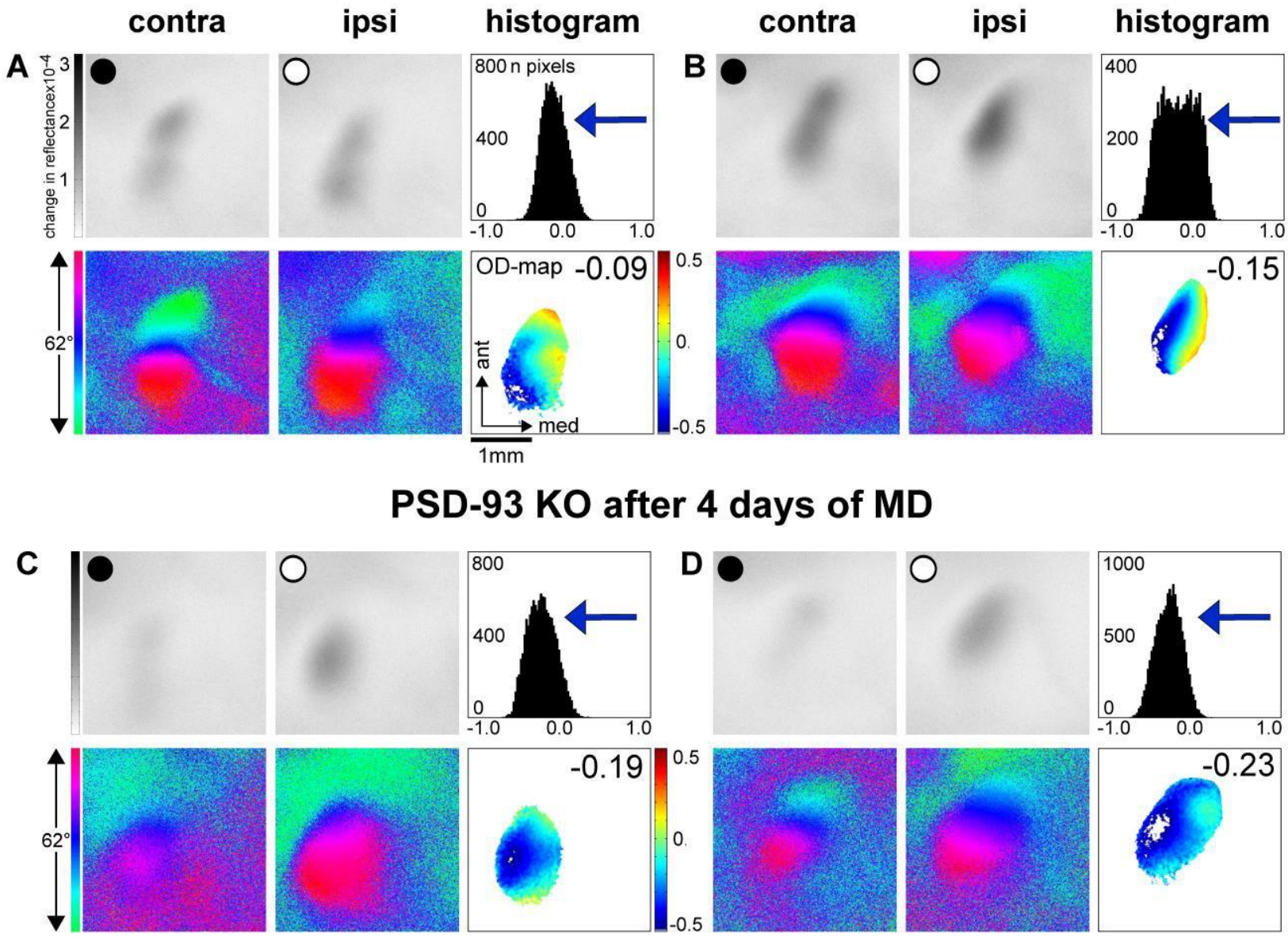

Figure 58: Representative examples of ocular dominance maps and indices in WT and PSD-93 KO mice after 4 days of MD obtained with optical imaging of intrinsic signals in vivo during the mid-critical period. Optical imaging maps of contralateral and ipsilateral cortical responses after visual stimulation of either eye with a horizontal moving bar (elevation) of $20^{\circ}$ are displayed from (A, B) two WT mice and (C, D) two PSD-93 KO mice after 4 days of $M D$, respectively. Grayscale coded response magnitude maps (top rows, expressed as fractional change in reflectance $\times 10^{-4}$ ) with black circles indicating monocular deprivation of the eye and white circles indicating that the respective eye remained open. Color-coded phase maps of retinotopy (bottom rows), histograms of the OD-scores (top right of panels) and color-coded OD-maps (right bottom including the average ODI value), are illustrated. After 4 days of $M D$, there was an OD-shift towards zero in both genotypes. Activity patches evoked by stimulation of the contralateral eye were equally strong to those after ipsi eye stimulation, 
the histogram shifted to the left and the average ODI was negative. Cold, blue colors prevailed in the 2dimensional OD-map, indicating ipsilateral dominance and hence ocular dominance plasticity.

In young standard cage raised WT mice during the mid CP, OD-plasticity could be observed after 4 days of MD with a strong OD-shift towards zero in comparison to WT mice without MD (ODI WT no MD: $0.39 \pm 0.02 ; n=3$; WT 4d MD: $-0.10 \pm 0.02 ; n=4 ; p<0.001 ; t$-test) as shown in Figure $56 \mathrm{~A}$. The OD-shift after MD was mediated by a decrease of deprived eye (contra) responses in V1 (contra WT no MD: $2.48 \pm 0.23$; WT 4d MD: $1.34 \pm 0.1 ; p<0.01$; t-test) (Figure 59 B), which is typical for juvenile OD-plasticity (Sawtell et al., 2003; Kaneko et al., 2008). In young PSD-93 KO mice, a similar effect could be observed. After 4 days of MD, PSD-93 KO mice showed a significant OD-shift (ODI KO no MD: $0.37 \pm 0.04 ; n=5 ;$ KO 4d MD: $-0.03 \pm 0.06$; $n=6 ; p<0.001 ; t$-test), which was also mediated by decreased deprived eye responses (contra KO no MD: $2.00 \pm 0.2 ; \mathrm{KO} 4 \mathrm{~d} \mathrm{MD}: 0.87 \pm 0.09 ; \mathrm{p}<0.001$; t-test). The OD-shift was similar to that of WT mice ( $p>0.05$, t-test; Figure 59 A, B). 


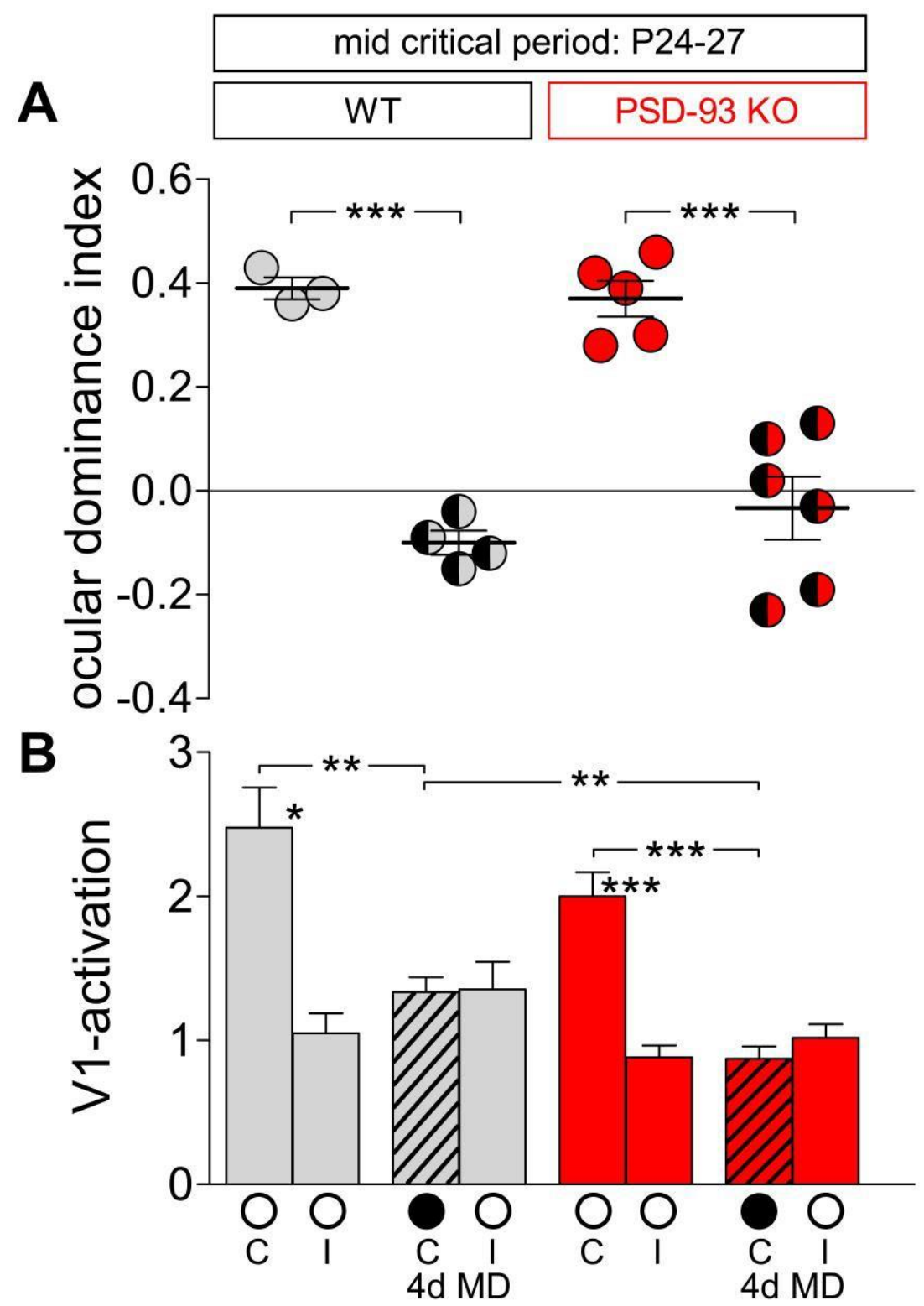

Figure 59: Ocular dominance indices and average response magnitudes of WT $(n=3)$ and PSD-93 KO mice without MD ( $n=5)$ and after four days of $M D(W T, n=4 / K O, n=6)$ during the mid-critical period. (A) A positive ODI indicates dominance of the contralateral eye, a negative ODI indicates ipsilateral dominance. Unicolored circles represent ODI values of individual animals without MD, half-filled circles represent individual animals after 4 days of MD; means are marked by thick horizontal lines and the standard deviation is illustrated with small horizontal lines. WT (gray) and PSD-93 KO mice (red) without MD showed ocular dominance of the contralateral eye and similar ODI values (t-test, $\mathrm{p}=0.51$ ). Four days of monocular deprivation in WT and PSD-93 KO mice induced a significant OD-shift towards the open eye (t-test, $p<0.001$ for both comparisons), which was not significantly different between both genotypes (t-test, $p=0.42$ ). (B) Average cortical responses expressed as a change in reflectance $x$ 10-4 by stimulation of the contralateral (C) or ipsilateral (I) eye in WT (gray) and PSD$93 \mathrm{KO}$ mice (red) without MD and after four days of MD. In all control animals without MD, cortical activation after visual stimulation of the contralateral eye was significantly higher than after ipsilateral eye stimulation (WT: $p<0.05$; KO: $p<0.001$; paired t-test), reflecting the dominance of the contralateral eye in mice. In both, 
WT and PSD-93 KO mice after four days of MD, response strength of the two eyes were no longer significantly different since the deprived eye responses were significantly reduced compared to controls (WT: $p<0.01 ; \mathrm{KO}: \mathrm{p}<$ 0.001 ; t-test). Number of tested animals from Leon Hosang $(n=12)$ and Sophia Stodieck $(n=6)$.

\subsection{OD-plasticity of WT and PSD-93 KO mice during late critical period}

Similar to WT and PSD-93 KO mice in the mid CP without MD, control animals in the late CP showed contralateral dominance with stronger visual cortical activation after contra eye stimulation than after visual stimulation of the ipsi eye. Representative 2-dimensional ocular dominance maps in the left V1 of both genotypes showed warm, red colors indicating contralateral dominance and all control mice exhibited positive ocular dominance indices (Figure 60).

\section{WT no MD}

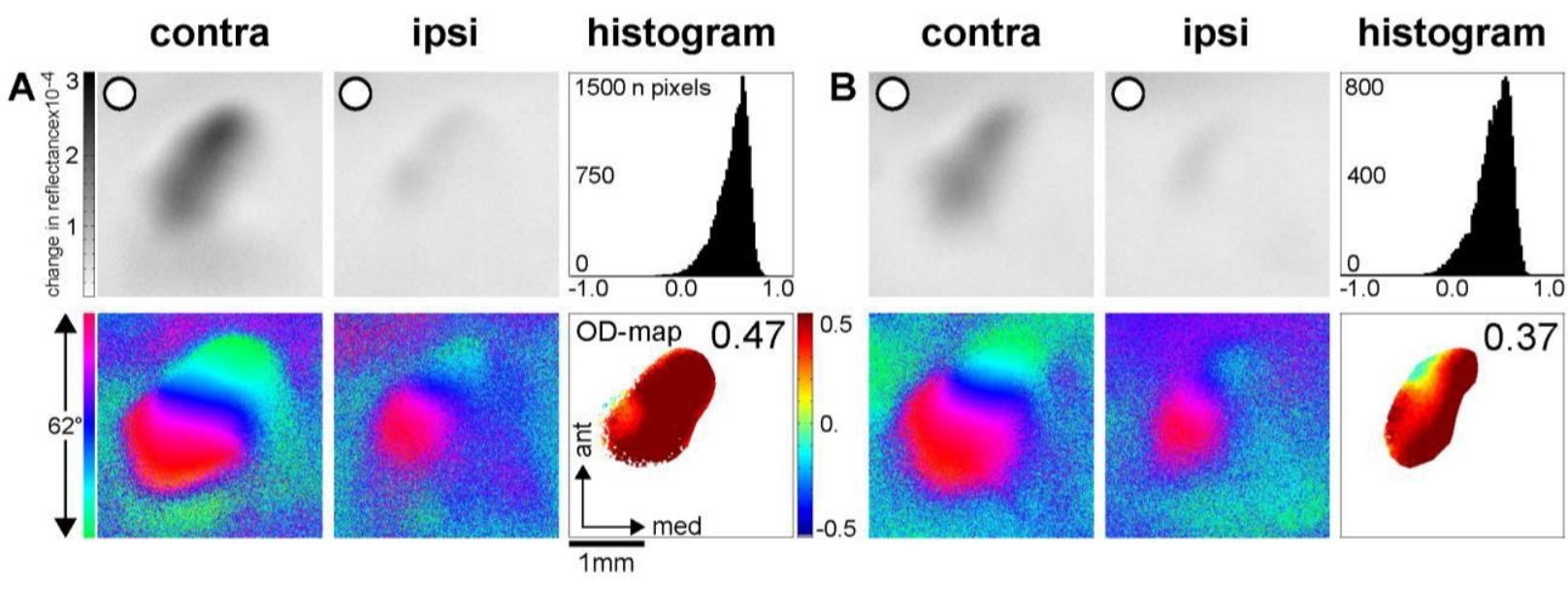

PSD-93 KO no MD
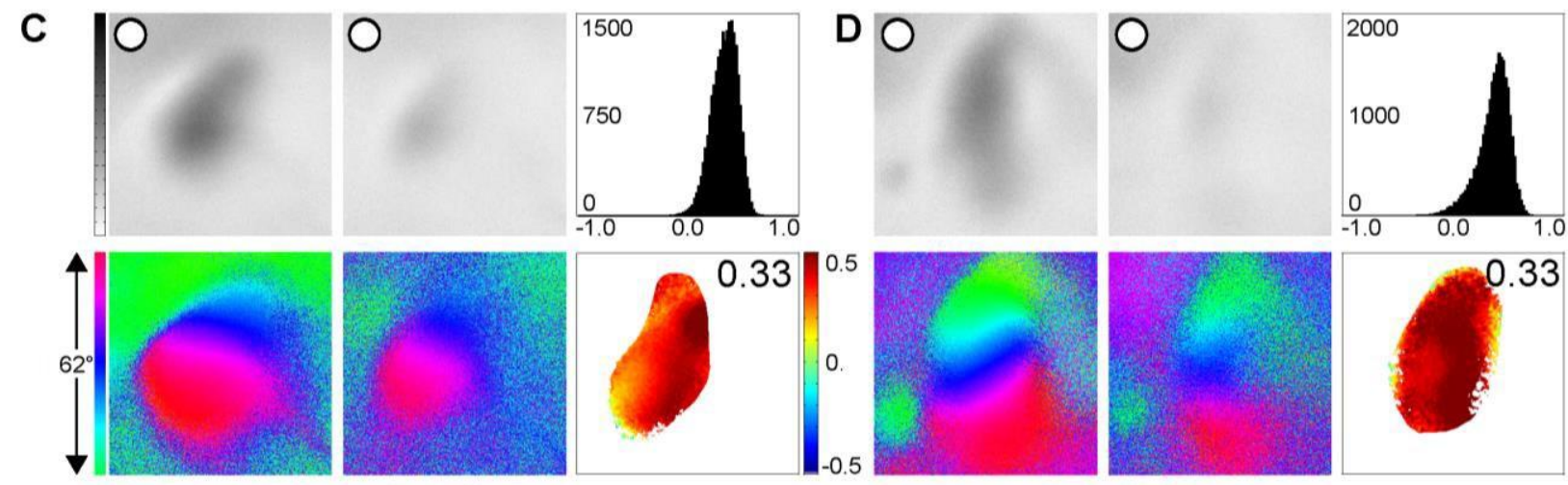

Figure 60: Representative examples of ocular dominance maps and indices in WT and PSD-93 KO mice without MD obtained with optical imaging of intrinsic signals in vivo during the late critical period. Optical imaging maps of contralateral and ipsilateral cortical responses to visual stimulation of either eye with a horizontal moving bar (elevation) of $20^{\circ}$ are displayed from (A, B) two WT mice and (C, D) two PSD-93 KO mice without MD, respectively. Grayscale coded response magnitude maps (top rows, expressed as fractional change in 
reflectance $\times 10^{-4}$ ), color-coded phase maps of retinotopy (bottom rows), histograms of the OD-scores (top right of panels) and color-coded OD-maps (right bottom including the average ODI value) are illustrated. In control mice of both genotypes, the activity patches evoked by stimulation on the contralateral (contra) eye were always darker than those evoked by ipsilateral (ipsi) eye stimulation. The average ODI was always positive and warm, red colors prevailed in the 2-dimensional OD-map, indicating contralateral dominance.

After 4 days of monocular deprivation, WT mice showed weakened cortical activity after visual stimulation of the contra eye, whereas ipsi eye stimulation activated the visual cortex stronger, hence showing OD-plasticity. The representative 2-dimensional ocular dominance maps in the left V1 of WT mice showed cold, blue colors, indicating ipsilateral dominance (Figure $61 \mathrm{~A}, \mathrm{~B}$ ). The histograms were shifted to the left and all mice exhibited negative ocular dominance indices. In contrast, this was not the case in PSD-93 KO mice. Here, 4 days of MD did not induce OD-plasticity and the cortical activation after visual stimulation of the contra eye remained higher than after visual stimulation of the ipsi eye. Additionally, the 2dimensional OD-maps showed warm, red colors indicating contralateral dominance and all mice showed positive ocular dominance indices (Figure $61 \mathrm{C}, \mathrm{D}$ ). 


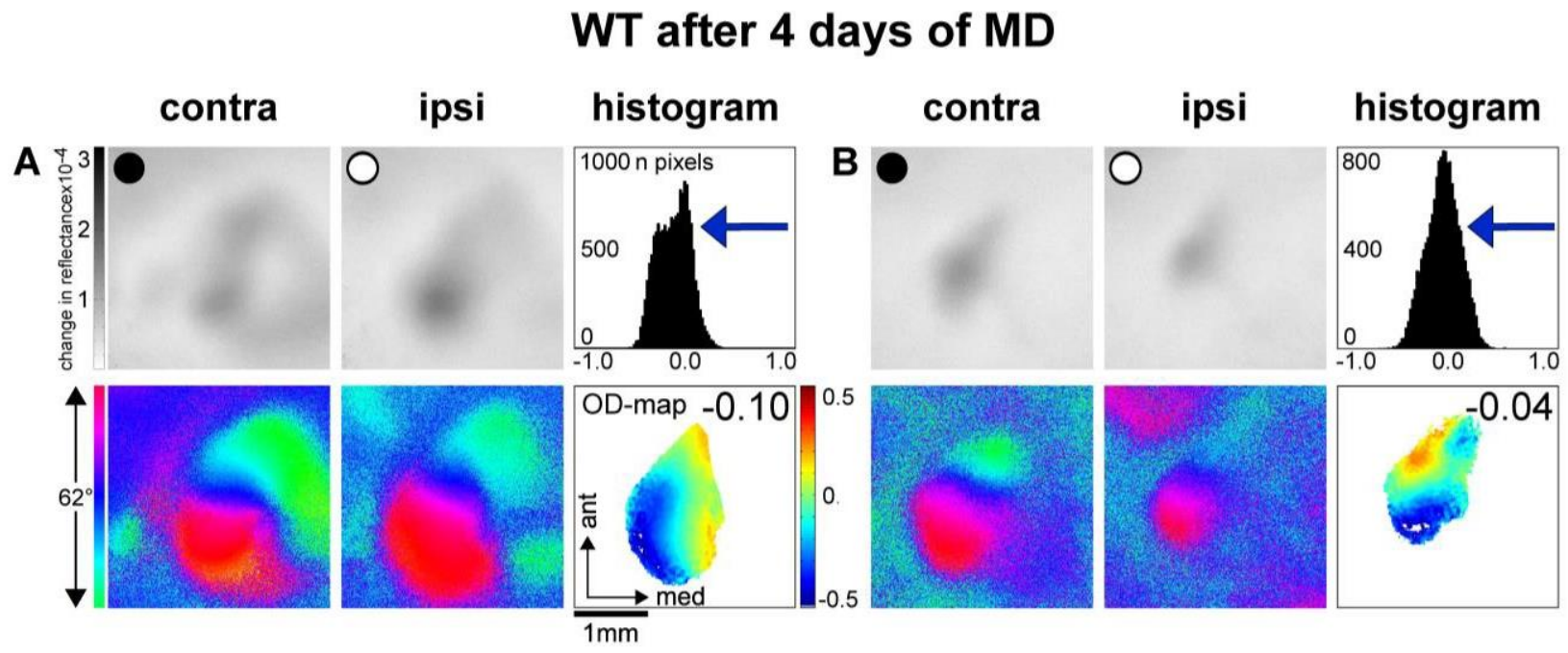

PSD-93 KO after 4 days of MD

C
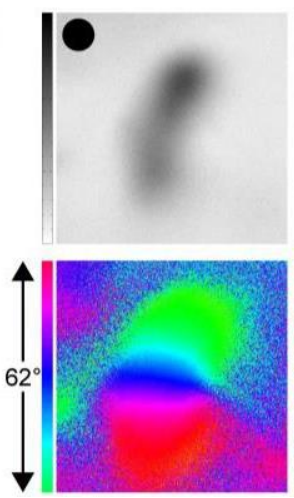
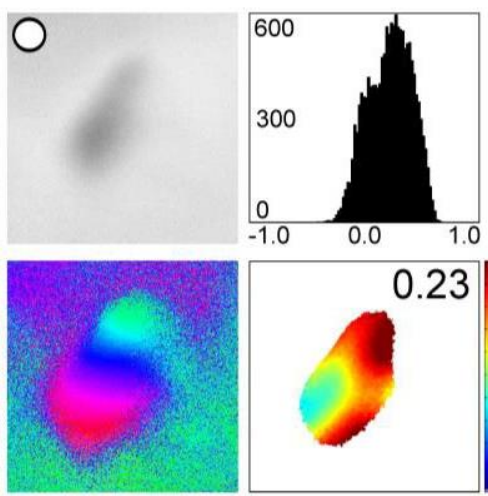

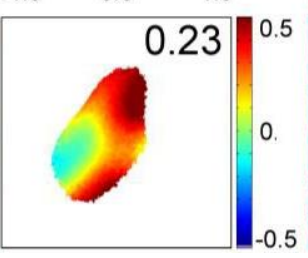

D
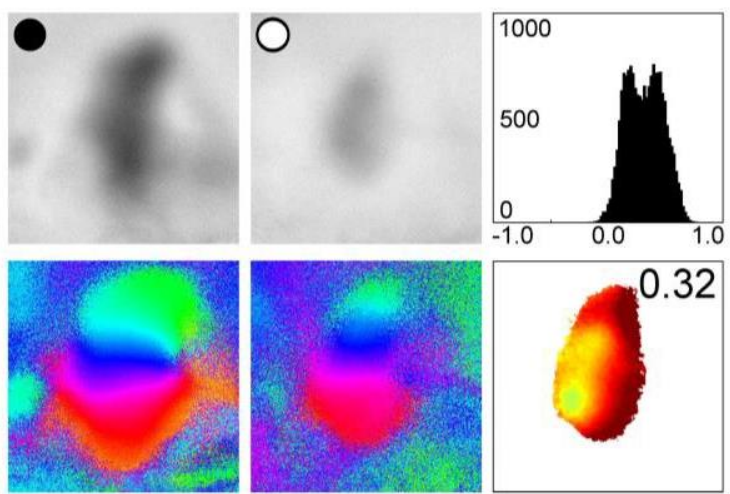
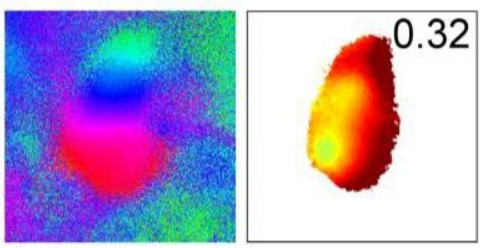

Figure 61: Representative examples of ocular dominance maps and indices of WT and PSD-93 KO mice after 4 days of MD obtained with optical imaging of intrinsic signals in vivo during the late-critical period. Optical imaging maps of contralateral and ipsilateral cortical responses to visual stimulation of either eye with a horizontal moving bar (elevation) of $20^{\circ}$ are displayed from (A, B) two WT mice and (C, D) two PSD-93 KO mice after 4 days of MD, respectively. Grayscale coded response magnitude maps (top rows, expressed as fractional change in reflectance $\times 10^{-4}$ ) with black circles indicating monocular deprivation of the eye and white circles indicating that the respective eye remained open. Color-coded phase maps of retinotopy (bottom rows), histograms of the OD-scores (top right of panels) and color-coded OD-maps (right bottom including the average ODI value), are illustrated. After 4 days of MD in WT mice, there was an OD-shift towards zero. Activity patches evoked by stimulation of the contralateral eye were equally strong to that after ipsi eye stimulation and the histogram shifted to the left. The average ODI was negative and cold, blue colors prevailed in the 2-dimensional OD-map, indicating ipsilateral dominance and hence ocular dominance plasticity. In contrast to that, in PSD-93 KO mice, the activity patches evoked by stimulation of the contra eye after 4 days of MD were still darker than those evoked by ipsi eye stimulation. The average ODI was always positive and warm, red colors prevailed in the 2-dimensional OD-map, indicating contralateral dominance and no ocular dominance plasticity. 
Quantification of the data revealed that already in the late CP (P 28 - 35), OD-plasticity was completely abolished in PSD-93 KO mice (ODI no MD: $0.32 \pm 0.02 ; n=5$; after 4 d MD: $0.28 \pm$ $0.02 ; n=6 ; p=0.17$; t-test), whereas it persists in WT mice after 4 days of MD (ODI no MD: $0.37 \pm 0.03 ; n=5$; after $4 d$ MD: $-0.01 \pm 0.02 ; n=7 ; p<0.001$; t-test) (Figure $62 \mathrm{~A}$ ). The ODshift in WT mice was similar to that of mice in the mid CP and also mediated by reduced contralateral eye responses (contra no MD: $1.85 \pm 0.14 ; n=5$; after 4 d MD: $1.15 \pm 0.18 ; n=$ 7; $p<0.05$; t-test), whereas ipsilateral eye responses remained unchanged (ipsi no MD: 0.81 $\pm 0.08 ; n=5 ;$ after 4 d MD: $1.04 \pm 0.14 ; n=7 ; p=0.23$; t-test) (Figure $62 \mathrm{~B}$ ).

These results already suggest that there is an earlier closure of the CP for OD-plasticity around P 28 when PSD-93 is lacking. 


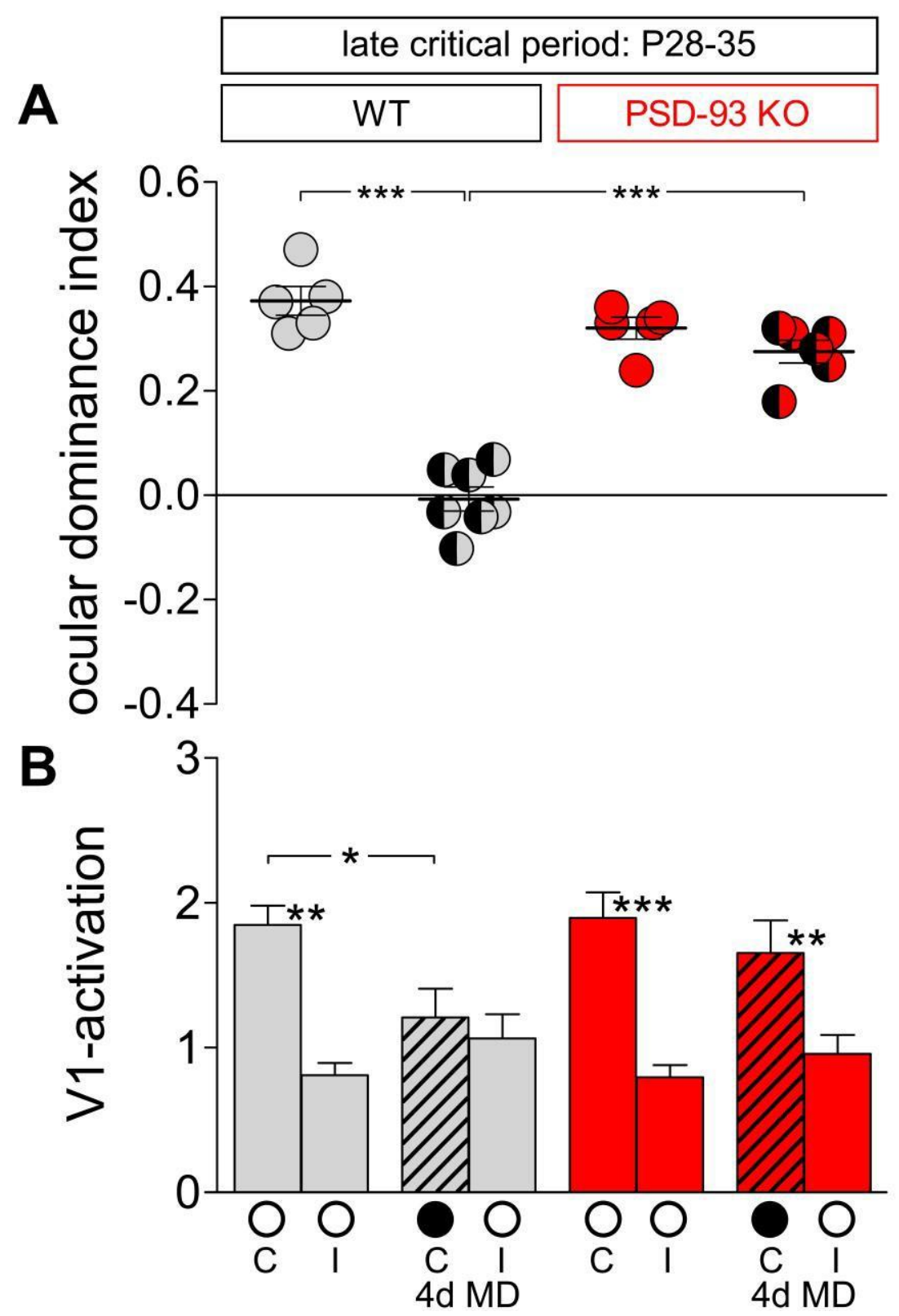

Figure 62: Ocular dominance indices and average response magnitudes of WT ( $=5$ ) and PSD-93 KO mice without MD ( $n=5)$ and after four days of $M D(W T, n=7 / K O, n=6)$ during the late-critical period. (A) A positive ODI indicates dominance of the contralateral eye, a negative ODI indicates ipsilateral dominance. Unicolored circles represent ODI values of individual animals without MD, half-filled circles represent individual animals after 4 days of MD; means are marked by thick horizontal lines and the standard deviation is illustrated with small horizontal lines. WT (gray) and PSD-93 KO mice (red) without MD showed ocular dominance of the contralateral eye and similar ODI values (t-test, $\mathrm{p}=0.17$ ). Four days of monocular deprivation in WT mice induced a significant OD-shift towards the open eye (t-test, $p<0.001$ ), which was significantly different from values of PSD-93 KO mice which did not show ocular dominance plasticity after MD (t-test, $\mathrm{p}<0.001)$. (B) Average cortical responses expressed as a change in reflectance $\times 10^{-4}$ by stimulation of the contralateral (C) or ipsilateral (I) eye in WT (gray) and PSD-93 KO mice (red) without MD and after four days of MD. In all control animals without MD, cortical activation after visual stimulation of the contralateral eye was significantly higher than after ipsilateral eye stimulation (WT: $p<0.01$; KO: $p<0.001$; paired t-test), reflecting the dominance of the contralateral eye in 
mice. In WT mice after four days of MD, response strength of the two eyes were no longer significantly different since the deprived eye responses were significantly reduced compared to controls (WT: $p<0.05 ; \mathrm{t}$-test). In contrast to that, cortical responses did not change in PSD-93 KO mice after MD and remained higher after contralateral eye stimulation in comparison to cortical activation after ipsilateral eye stimulation $(p<0.01$; paired t-test). Number of tested animals from Leon Hosang $(n=11)$ and Sophia Stodieck $(n=12)$.

\subsection{Dark rearing does not prevent the early maturation of PSD-93 KO mice}

Standard cage WT mice raised in complete darkness (dark rearing (DR)) show a prolonged CP for OD-plasticity. Funahashi et al. (2013) could show that during the development of mice, silent synapses were largely present before eye opening. This number of silent synapses significantly decreases during the critical period of OD-plasticity and they are almost absent in adulthood (Huang et al., 2015). However, this decline of silent synapse numbers during development was prevented by dark rearing (Funahashi et al., 2013).

According to these findings, the fraction of silent synapses in dark reared PSD-93 KO mice was investigated (Favaro; Schlüter laboratory, ENI, Göttingen). It was found that even dark rearing cannot prevent the rapid decline of silent synapse numbers in PSD-93 KO mice that were already largely absent during the CP (Figure 63).

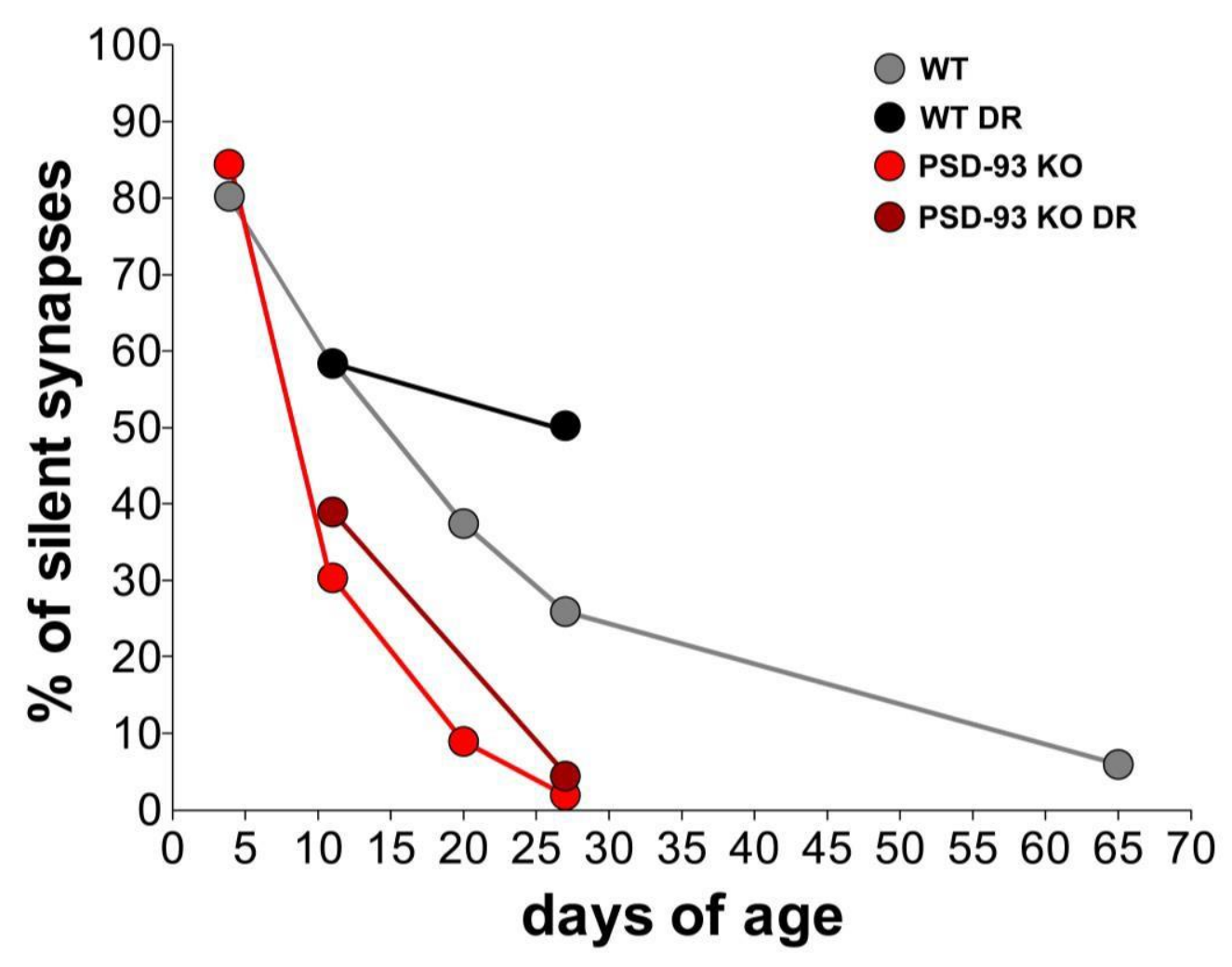


Figure 63: Number of AMPA receptor silent synapses in percent (\%) measured in the VC of WT and PSD-93 KO mice after dark rearing from birth. The number of AMPA receptor silent synapses is gradually decreasing in WT mice (gray) from $80 \pm 0.05 \%$ (P $3-P$ 5) to $58.6 \pm 0.04 \%$ (P $10-P 12$ ) and further to $37.5 \pm 0.06 \%$ (P 19- P21). During the critical period, the numbers of AMPA silent synapses reach $26 \pm 0.05 \%$ (P $25-$ P 30) which further decrease even below $9 \%$ after the critical period. In contrast, the numbers of AMPA receptor silent synapses remained high in WT mice (black) when reared in darkness (DR) about $49 \pm 0.05 \%$ during the critical period. In PSD-93 KO mice, AMPA receptor silent synapses were abundant before eye opening at P $3-$ P 5 (85 $\pm 0.04 \%$ ) but already declined to $30 \pm 0.06 \%$ between P $10-P 12$. Interestingly, already at the very beginning of the critical period (P 20), AMPA receptor silent synapses dropped to $9 \pm 0.05 \%$ in PSD-93 KO mice and were nearly absent at P 25 ( $2 \pm 0.06 \%)$. In PSD-93 KO mice, the fraction of silent synapses decreased over the time course of development which could not be prevented by dark rearing as shown for WT mice. The numbers of silent synapses decreased from $39.5 \pm 0.07 \%$ at P $10-$ P 12 to $5 \pm 0.05 \%$ already at P 25. Data obtained by Favaro (unpublished data).

Simultaneously, I performed optical imaging of intrinsic signals in dark reared PSD-93 KO and WT control mice without MD and after 4 days of MD between P 28 - 35 during the late CP. Dark reared WT and PSD-93 KO control mice without MD showed contralateral dominance with stronger visual cortical activation after contra eye stimulation than after visual stimulation of the ipsi eye. Representative 2-dimensional ocular dominance maps in the left V1 of both genotypes showed warm, red colors indicating contralateral dominance and all control mice exhibited positive ocular dominance indices (Figure 64). 


\section{WT dark rearing no MD}

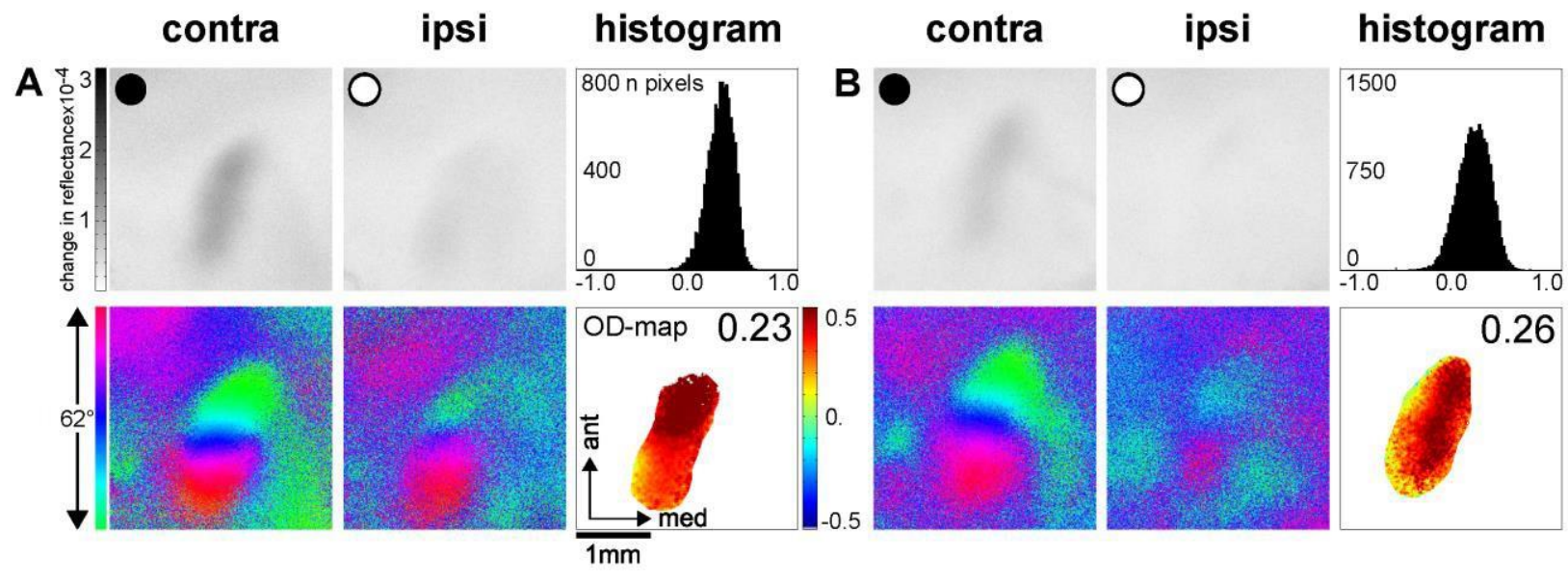

PSD-93 KO dark rearing no MD
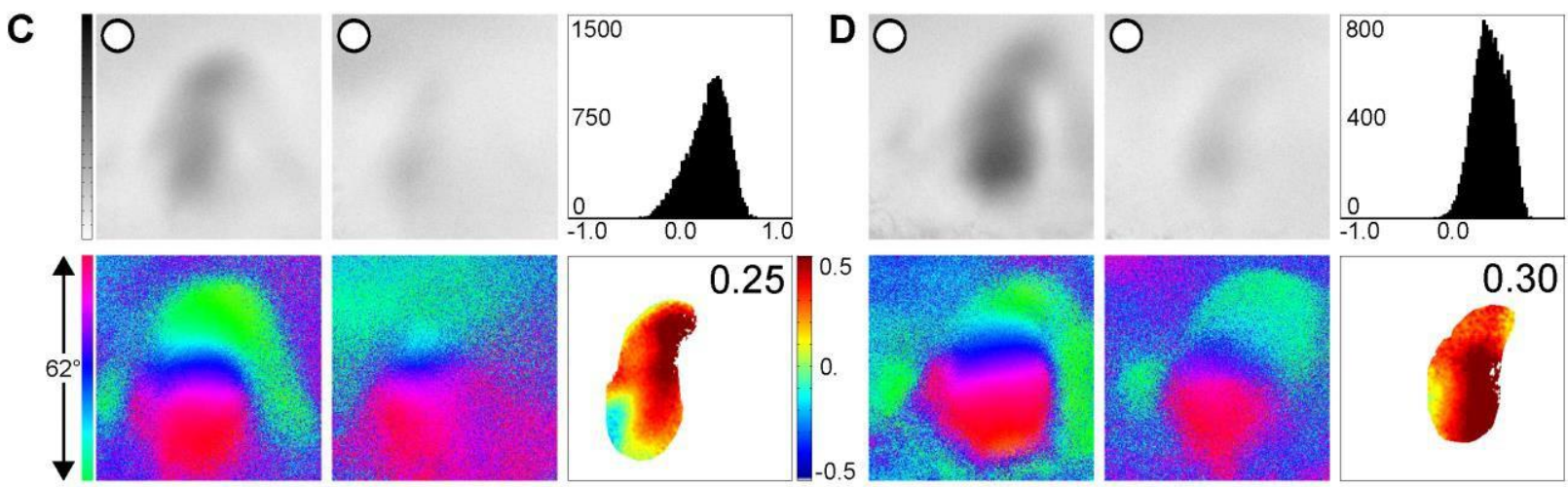

Figure 64: Representative examples of ocular dominance maps and indices in WT and PSD-93 KO mice reared in darkness without MD obtained with optical imaging of intrinsic signals in vivo during the late critical period. Optical imaging maps of contralateral and ipsilateral cortical responses to visual stimulation of either eye with a horizontal moving bar (elevation) of $20^{\circ}$ are displayed from (A, B) two WT mice and (C, D) two PSD-93 KO mice without MD, respectively. Grayscale coded response magnitude maps (top rows, expressed as fractional change in reflectance $\times 10^{-4}$ ), color-coded phase maps of retinotopy (bottom rows), histograms of the OD-scores (top right of panels) and color-coded OD-maps (right bottom including the average ODI value), are illustrated. In control mice of both genotypes, the activity patches evoked by stimulation of the contralateral (contra) eye were always darker than those evoked by ipsilateral (ipsi) eye stimulation. The average ODI was always positive and warm, red colors prevailed in the 2-dimensional OD-map, indicating contralateral dominance.

After 4 days of monocular deprivation, dark reared WT mice showed OD-plasticity with weakened cortical activation after visual stimulation of the contra eye, whereas ipsi eye stimulation activated the cortex stronger. The representative 2-dimensional ocular dominance maps in the left visual cortex of WT mice showed cold, blue colors indicating 
ipsilateral dominance and the histograms were shifted to the left. All tested WT mice exhibited negative ocular dominance indices and hence OD-plasticity (Figure $65 \mathrm{~A}, \mathrm{~B}$ ).

In contrast to dark reared WT mice, this was not the case in dark reared PSD-93 KO mice. Here, 4 days of MD could not induce OD-plasticity and the cortical activation after visual stimulation of the contra eye remained higher than after visual stimulation of the ipsi eye. Additionally, the 2-dimensional OD-map showed warm, red colors indicating contralateral dominance and all mice exhibited positive ocular dominance indices (Figure 65 C, D).

\section{WT dark rearing after 4 days of MD}

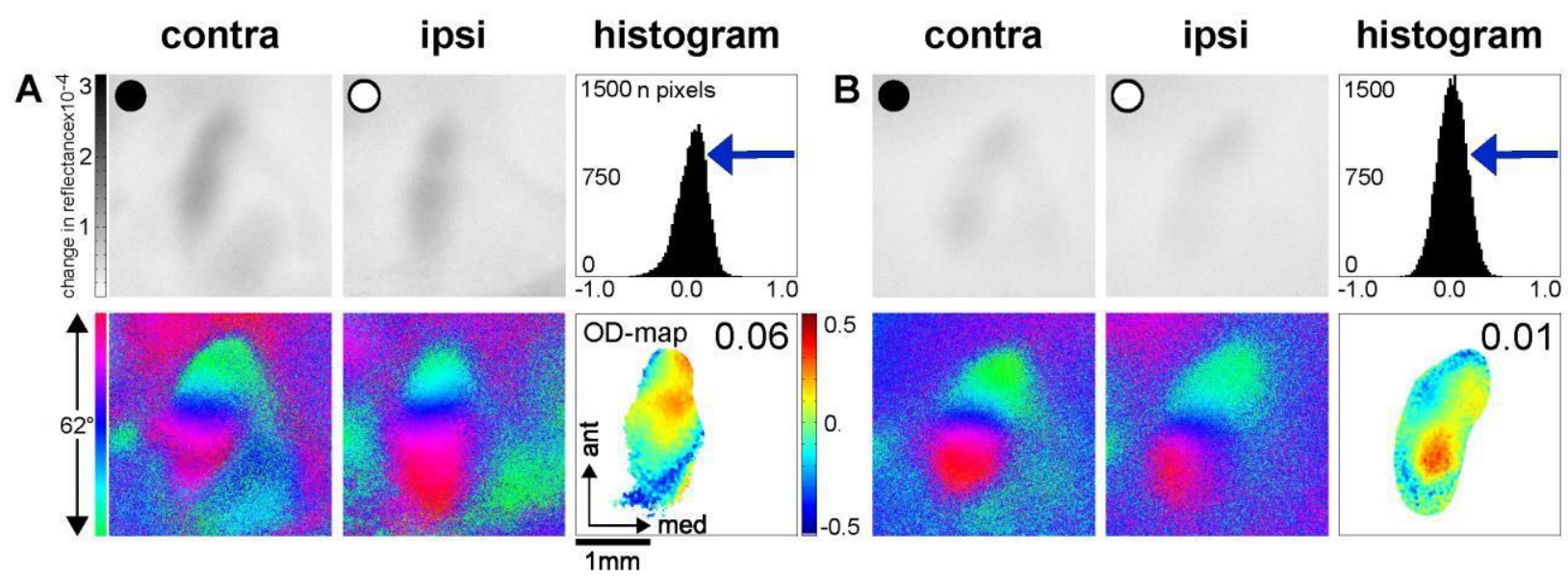

PSD-93 KO dark rearing after 4 days of MD
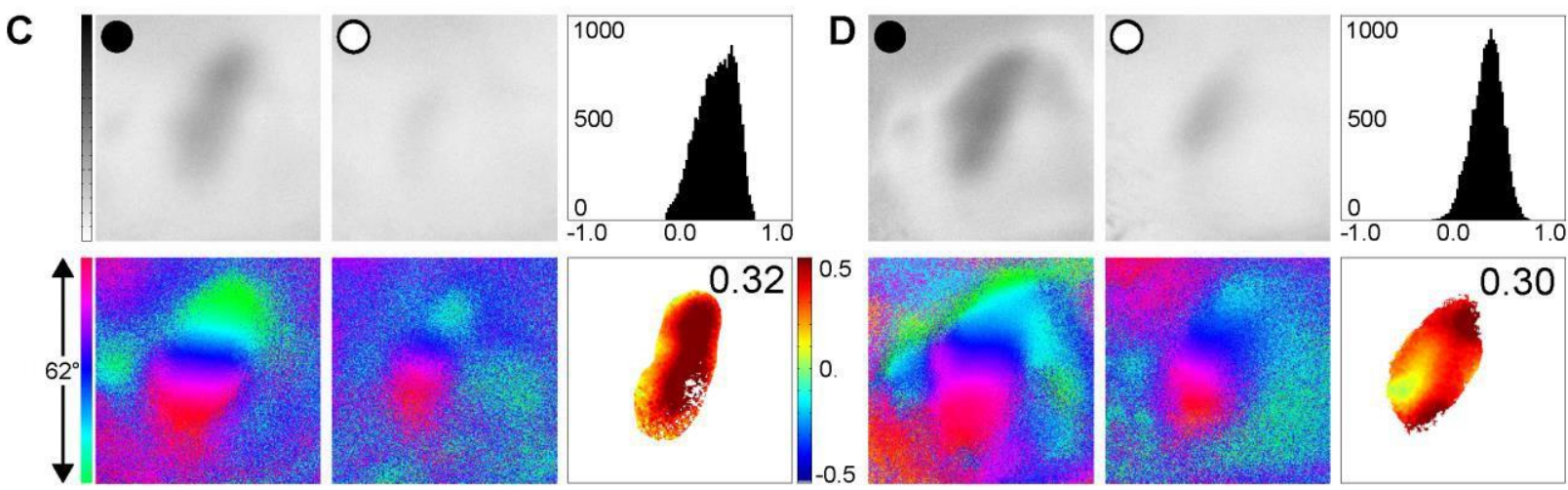

Figure 65: Representative examples of ocular dominance maps and indices in WT and PSD-93 KO mice reared in darkness after 4 days of MD obtained with optical imaging of intrinsic signals in vivo during the late critical period. Optical imaging maps of contralateral and ipsilateral cortical responses to visual stimulation of either eye with a horizontal moving bar (elevation) of $20^{\circ}$ are displayed from (A, B) two WT mice and (C, D) two PSD$93 \mathrm{KO}$ mice after 4 days of MD, respectively. Grayscale coded response magnitude maps (top rows, expressed as fractional change in reflectance $\times 10^{-4}$ ) with black circles indicating monocular deprivation of the eye and white circles indicating that the respective eye remained open. Color-coded phase maps of retinotopy (bottom rows), histograms of the OD-scores (top right of panels) and color-coded OD-maps (right bottom including the average ODI value), are illustrated. After 4 days of MD in WT mice, there was an OD-shift towards zero, the 
activity patches evoked by stimulation of the contra eye were equally strong to that after ipsi eye stimulation, the histogram shifted to the left and cold, blue colors prevailed in the 2-dimensional OD-map. The average ODI was negative, indicating ipsilateral dominance and hence ocular dominance plasticity. In contrast to that, in PSD$93 \mathrm{KO}$ mice, the activity patches evoked by stimulation of the contra eye after MD were still darker than those evoked after ipsi eye stimulation. The average ODI was positive and warm, red colors prevailed in the 2dimensional OD-map, indicating contralateral dominance and no ocular dominance plasticity.

The results reveal that PSD-93 KO mice do not show OD-plasticity even after dark rearing from birth during the late CP (P 28 - 35) (ODI no MD: $0.27 \pm 0.02 ; n=3$; after 4 d MD: $0.24 \pm 0.02$; $\mathrm{n}=6 ; \mathrm{p}=0.5$; t-test), whereas dark reared WT mice showed OD-plasticity with a strong ODshift towards zero (ODI no MD: $0.26 \pm 0.03 ; n=6$; after 4 d MD: $0.00 \pm 0.02 ; n=5 ; p<0.001$; t-test) as shown in Figure $67 \mathrm{~A}$.

This strong OD-shift in dark reared WT mice was mediated by a reduction of deprived eye responses (WT late CP contra no MD: $1.14 \pm 0.15 ; n=6$; WT late CP contra 4d MD: $0.7 \pm 0.14$; $\mathrm{n}=5 ; \mathrm{p}<0.05 ; \mathrm{t}$-test), whereas ipsilateral eye evoked responses in V1 remained unchanged (WT late CP ipsi no MD: $0.56 \pm 0.11 ; n=6$; WT late CP ipsi 4d MD: $0.61 \pm 0.1 ; p=0.74$; t-test) (Figure $67 \mathrm{~B}$ ).

This OD-plasticity persisted in WT mice even beyond the CP between P $40-50$. Dark reared WT mice with 4 days of MD beyond the CP showed OD-plasticity with weakened cortical activation after visual stimulation of the contra eye, whereas ipsi eye stimulation activated the cortex equally strong. The representative 2-dimensional ocular dominance maps in the left visual cortex of DR WT mice beyond the CP showed cold, blue colors indicating ipsilateral dominance, the histograms were shifted to the left and all mice exhibited negative ocular dominance indices (Figure $65 \mathrm{~A}, \mathrm{~B}$ ). In contrast, age-matched WT mice which were kept under normal light conditions still showed contralateral dominance after 4 days of MD with stronger visual cortical activation after contra eye stimulation than after visual stimulation of the ipsi eye. Representative 2-dimensional ocular dominance maps in the left V1 showed warm, red colors indicating contralateral dominance and all light reared WT mice exhibited positive ocular dominance indices (Figure $65 \mathrm{C}, \mathrm{D}$ ). 


\section{WT dark rearing after 4 days of MD - beyond critical period}
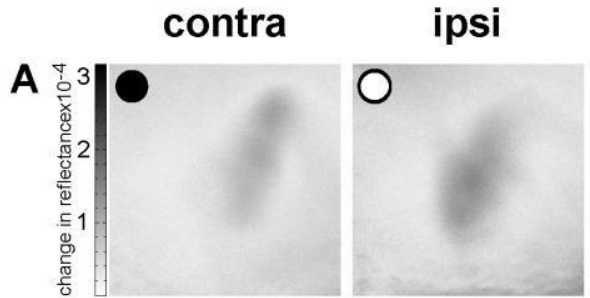

histogram

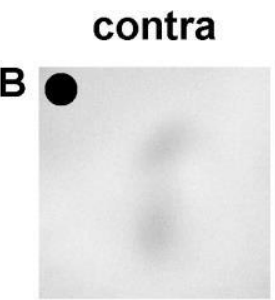

ipsi

histogram
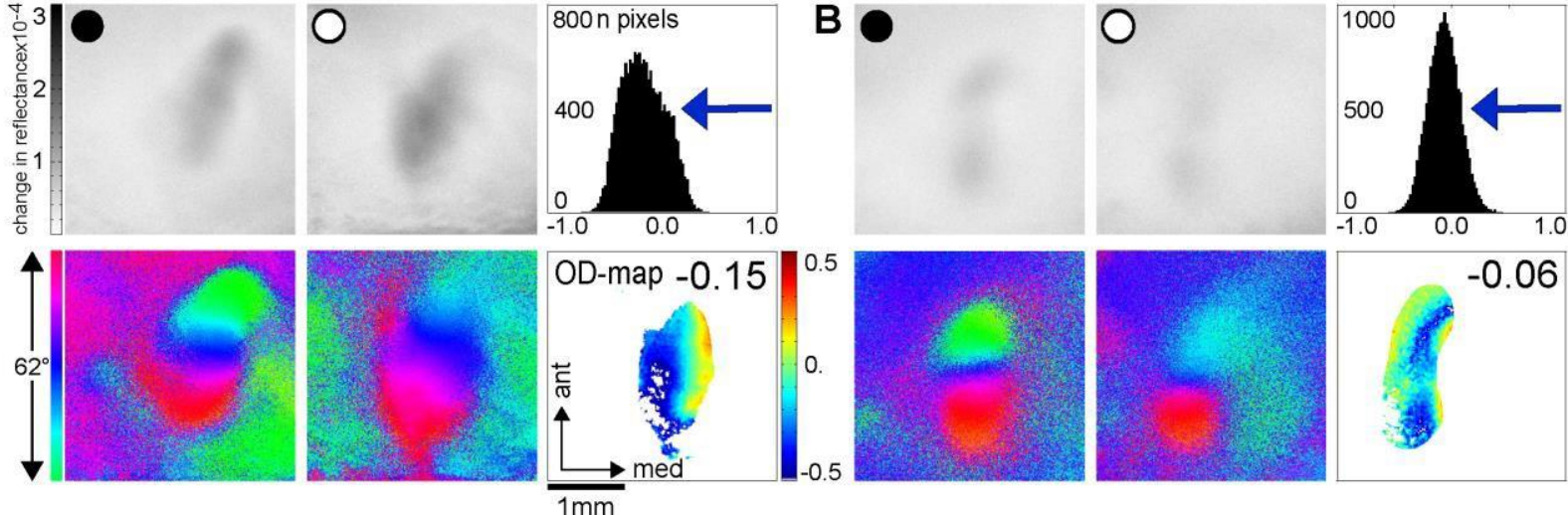

WT light rearing after 4 days of MD - beyond critical period
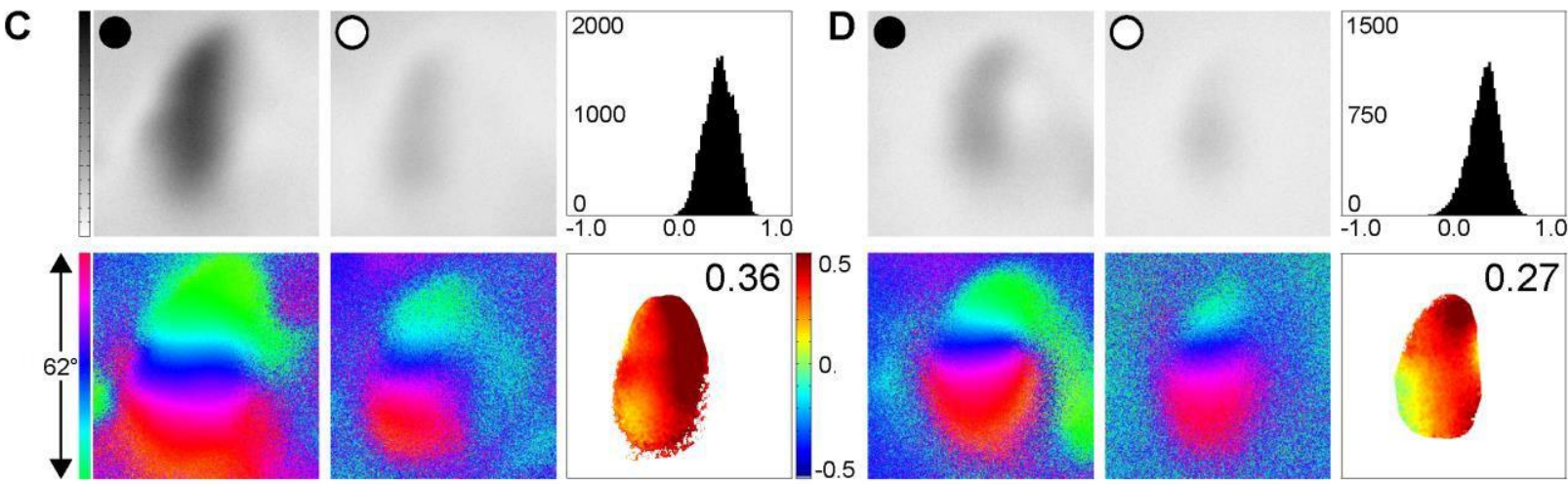

Figure 65: Representative examples of ocular dominance maps and indices of light reared and dark reared WT mice after 4 days of MD beyond the critical period for OD-plasticity (P 40 - 50). Optical imaging maps of contralateral and ipsilateral cortical responses to visual stimulation of either eye with a horizontal moving bar (elevation) of $20^{\circ}$ are displayed from (A, B) two dark reared WT mice and (C, D) two normally light reared WT mice after 4 days of MD, respectively. Grayscale coded response magnitude maps (top rows, expressed as fractional change in reflectance $\times 10^{-4}$ ) with black circles indicating monocular deprivation of the eye and white circles indicating that the respective eye remained open. Color-coded phase maps of retinotopy (bottom rows), histograms of the OD-scores (top right of panels) and color-coded OD-maps (right bottom including the average ODI value), are illustrated. After 4 days of MD in dark reared WT mice, there was an OD-shift towards zero. Activity patches evoked by stimulation of the contralateral eye were equally strong to that after ipsi eye stimulation and the histogram shifted to the left. The average ODI was negative and cold, blue colors prevailed in the 2-dimensional OD-map, indicating ipsilateral dominance and hence ocular dominance plasticity. In contrast to that, in normal light reared control mice, the activity patches evoked by stimulation of the contra eye after MD were still darker than those evoked after ipsi eye stimulation. The average ODI was always positive and warm, red colors prevailed in the 2-dimensional OD-map, indicating contralateral dominance and no ocular dominance plasticity. 
Quantification of the data revealed that all DR WT mice beyond the critical period showed ODplasticity after only 4 days of MD (ODI after $4 \mathrm{~d} M D$ : $-0.01 \pm 0.05 ; n=5)$, whereas age matched light-reared control mice did not exhibit OD-plasticity after 4 days of MD (ODI: $0.28 \pm 0.03 ; n$ $=5 ; p<0.001 ;$ t-test), as shown in Figure $67 \mathrm{~A}$. This is in line with studies showing similar plasticity after dark rearing in WT mice (Review: Hooks \& Chen, 2007), cats (Mower et al., 1981; Mower et al., 1991; Cynader, 1983) and rats (Fagiolini et al., 1994, Morales et al., 2002) among others.

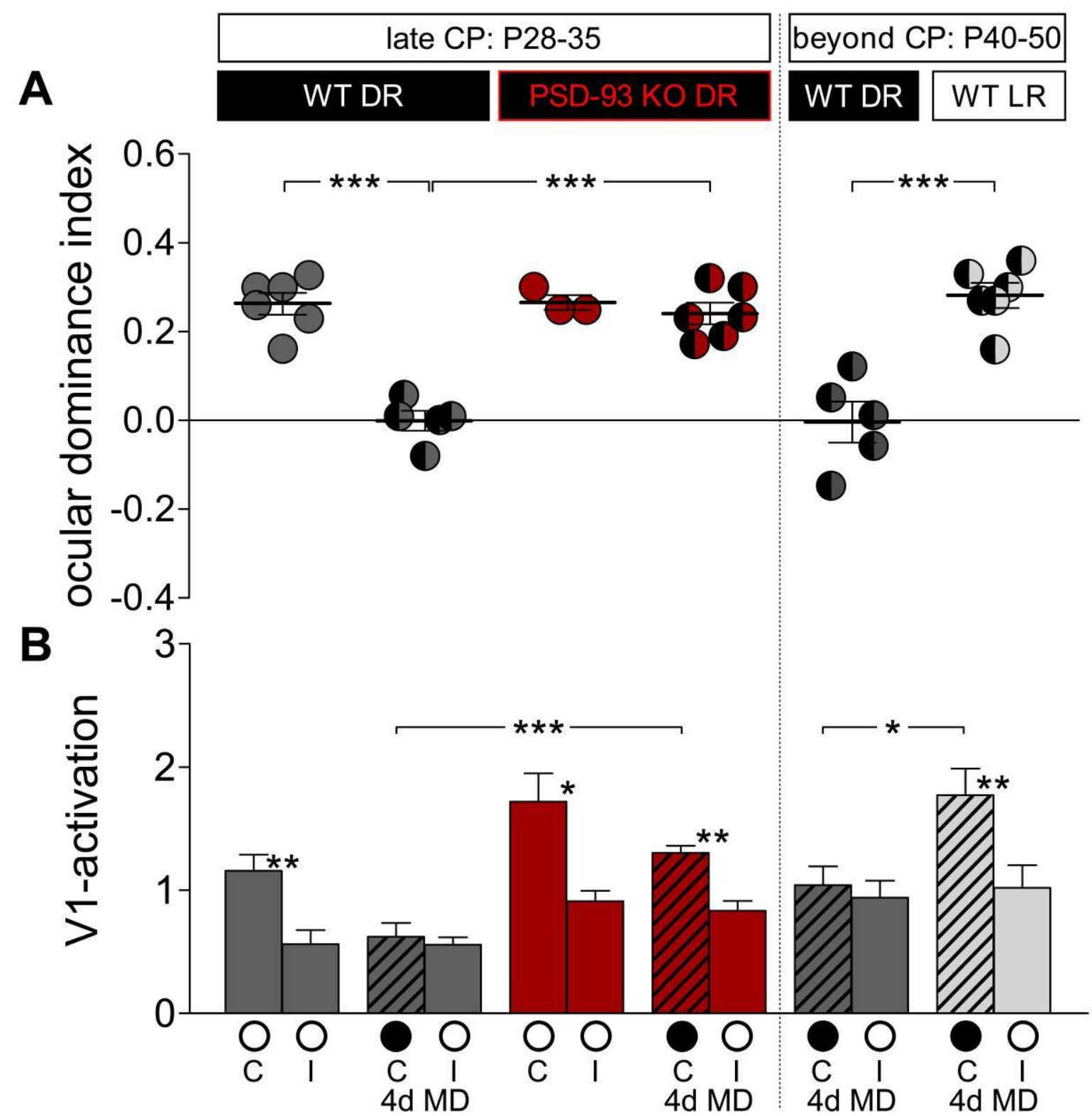

Figure 67: Ocular dominance indices and average response magnitudes of dark reared WT $(n=6)$ and PSD-93 KO mice without MD $(n=3)$ and after four days of MD (WT, $n=5 / K O, n=6)$ during the late-critical period and dark reared $(n=5)$ and light reared $(n=6)$ WT mice beyond the critical period after four days of MD. (A) A positive ODI indicates dominance of the contralateral eye, a negative ODI indicates ipsilateral dominance. 
Unicolored circles represent ODI values of individual animals without MD, half-filled circles represent individual animals after 4 days of MD; means are marked by thick horizontal lines and the standard deviation is illustrated with small horizontal lines. WT (gray) and PSD-93 KO mice (dark red) without MD showed ocular dominance of the contralateral eye and similar ODI values ( $t$-test, $p=0.93$ ). Four days of monocular deprivation in WT ( $t$-test, $p$ $<0.001)$ but not in PSD-93 KO ( $p=0.5)$ mice induced a significant OD-shift towards the open eye which was significantly different between both genotypes (t-test, $p<0.001$ ). After the critical period, dark rearing prolonged the sensitive phase for ocular dominance plasticity in WT mice, which still showed an OD-shift after 4 days of MD in comparison to normal light reared WT mice $(p<0.001)$. (B) Average cortical responses expressed as a change in reflectance $\times 10^{-4}$ by stimulation of the contralateral (C) or ipsilateral (I) eye in WT (gray) and PSD$93 \mathrm{KO}$ mice (dark red) without MD and after four days of MD. In all control animals without MD, cortical activation after visual stimulation of the contralateral eye was significantly higher than after ipsilateral eye stimulation (WT: $p<0.01$; KO: $p<0.05$; paired t-test), reflecting the dominance of the contralateral eye in mice. Only in WT mice after four days of MD, the response strengths of the two eyes were no longer significantly different since the deprived eye responses were slightly reduced compared to controls (WT: $p=0.052, t$-test). In contrast, in dark reared PSD-93 KO mice, cortical activation was still higher after visual stimulation of the contra eye compared to the ipsi eye ( $p<0.01$; paired t-test). Comparison of V1-activation in WT mice after the critical period revealed that dark reared mice had significantly reduced deprived eye responses in comparison to light reared mice after 4 days of MD ( $p<0.05$; t-test).

Thus, characteristic features of juvenile OD-plasticity were present in WT mice during the CP and even beyond the $\mathrm{CP}$, if reared in darkness. In contrast, OD-plasticity was completely absent in PSD-93 KO mice after P 27. These findings indicate that the critical period plasticity is closing earlier in PSD-93 KO mice and cannot be rescued by dark rearing. These results indicate that the faster maturation of PSD-93 KO mice is not an experience-dependent process since it was independent of visual experience.

To examine if plasticity can be preserved in PSD-93 KO mice during the critical period by other means, OD-plasticity was investigated in PSD-93 KO and WT mice after voluntary physical exercise.

\subsection{Running does not preserve OD-plasticity in PSD-93 KO mice}

It was shown that raising mice in an enriched environment (EE) that provides more social interactions, voluntary physical exercise with running wheels, and cognitive stimulation compared to mice raised in standard cages (SC), preserves juvenile-like OD-plasticity into late adulthood (Greifzu et al., 2014). Additionally, it was shown that not all components of the EE 
are needed to preserve OD-plasticity. Physical exercise alone (running in a wheel) is already sufficient to prolong plasticity and the sensitive phase for OD-plasticity in mice after 7 days of MD (Kalogeraki et al., 2014). Even running only during the MD-period of 7 days restored juvenile-like OD-plasticity in adult standard cage raised mice.

To test whether physical exercise e.g. running alone would also preserve OD-plasticity in PSD$93 \mathrm{KO}$ mice, mice were raised in a cage with a running wheel (RW) equipped with a counter which recorded the turns of the wheel. The counter was checked daily and the average numbers of turns was calculated for every mouse dividing the counted numbers of turns by the number of mice living in each cage (2-5 mice per cage). The average amount of RW-turns for PSD-93 KO mice was 7.452 turns per day per mouse, corresponding to $0.98 \pm 0.074 \mathrm{~km}$ per day per mouse. The diameter of the running wheel was $12.8 \mathrm{~cm}$ which was used to convert the turns of the wheel into kilometers. Between P 24 - 30, mice were either monocularly deprived for four days $(n=5)$ or without MD $(n=3)$ and OptoMotry was performed daily until optical imaging of intrinsic signals was performed between P 28 - 34.

In RW PSD-93 KO mice without MD (control group), visual stimulation of the contralateral (contra) eye induced stronger visual cortical activation than after visual stimulation of the ipsilateral (ipsi) eye, which means the activity patches obtained from the V1 after stimulation of the contra eye were always darker than those after ipsi eye stimulation. This phenomenon indicates the typical contralateral dominance in the binocular region of V1 in mice (Figure 68 A, B). Representative 2-dimensional ocular dominance maps in the left visual V1 showed warm, red colors indicating contralateral dominance and all control mice exhibited positive ODIs (Figure 68 A, B).

Surprisingly, even voluntary physical exercise and 4 days of MD did not induce any change in PSD-93 KO mice during the late CP. These mice still showed a positive ODI and a stronger V1activation after visual stimulation of the contra eye than after visual stimulation of the ipsi eye (Figure 68 C, D). 


\section{PSD-93 KO running wheel no MD}

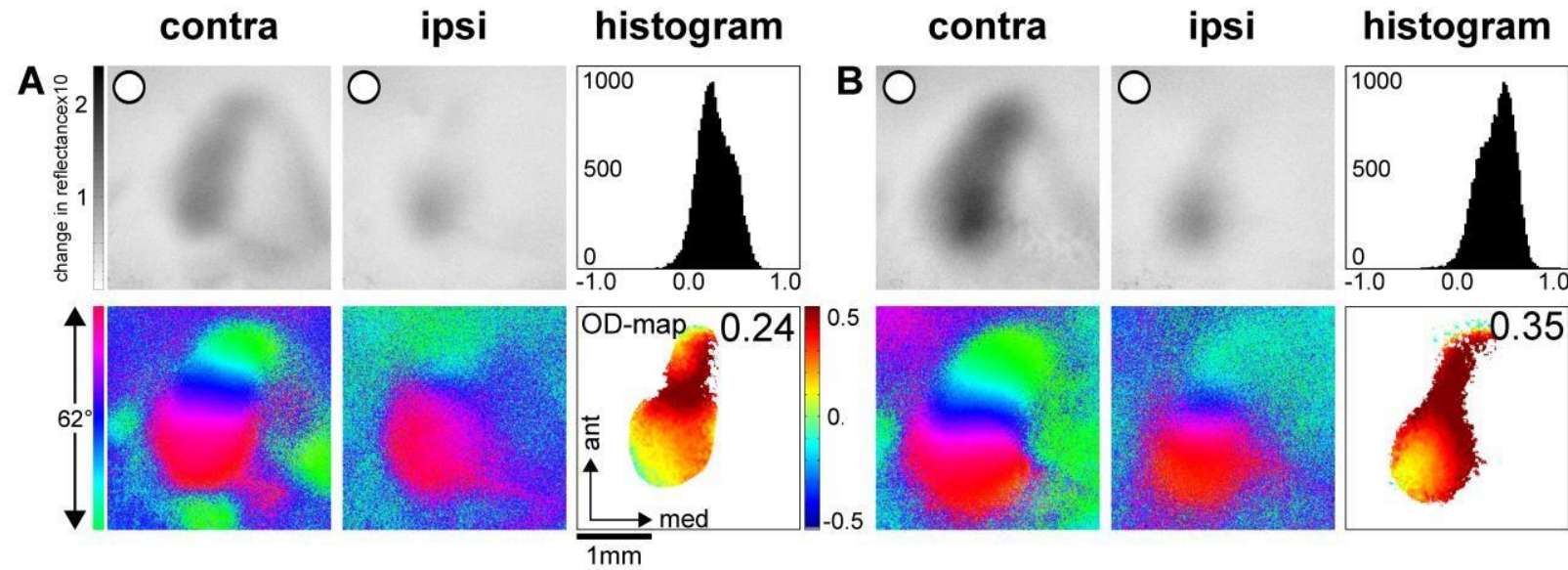

PSD-93 KO running wheel 4 days of MD
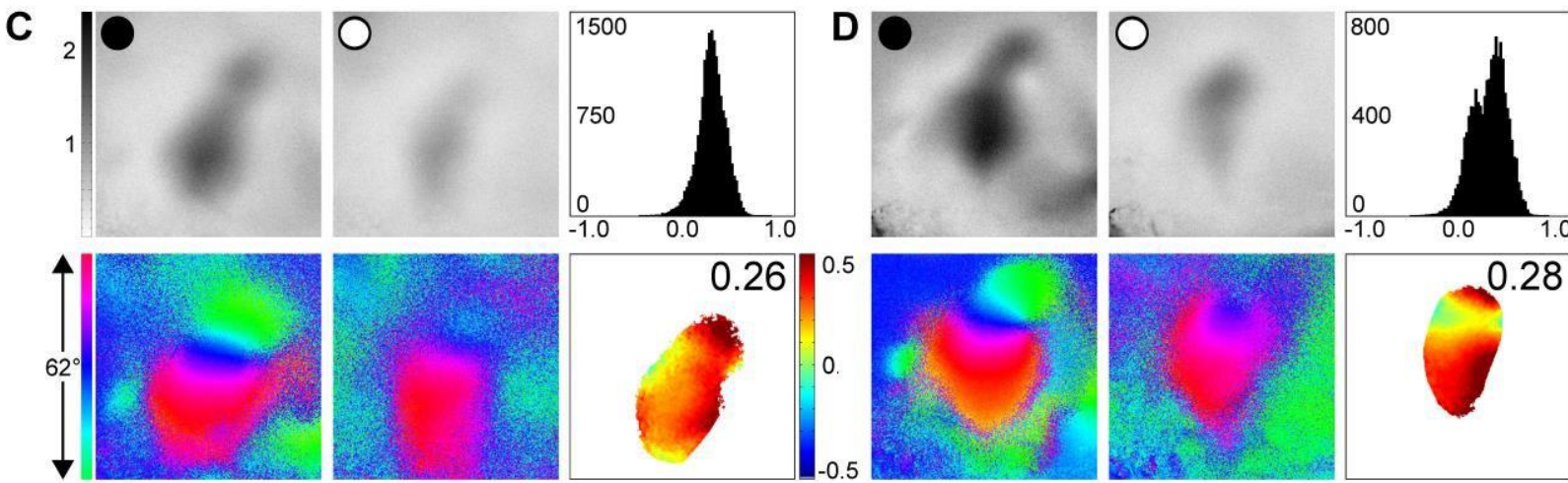

Figure 68: Representative examples of ocular dominance maps and indices of PSD-93 KO mice raised in a cage with a running wheel obtained with optical imaging of intrinsic signals in vivo during the late-critical period. Optical imaging maps of contralateral and ipsilateral cortical responses to visual stimulation of either eye with a horizontal moving bar (elevation) of $20^{\circ}$ are displayed from (A, B) two RW PSD-93 KO mice without MD and (C, D) two RW PSD-93 KO mice after 4 days of MD, respectively. Grayscale coded response magnitude maps (top rows, expressed as fractional change in reflectance $\times 10^{-4}$ ), color-coded phase maps of retinotopy (bottom rows), histograms of the OD-scores (top right of panels) and color-coded OD-maps (right bottom including the average ODI value), are illustrated. In control mice without MD as well as in mice after 4 days of MD, the activity patches evoked by stimulation on the contralateral (contra) eye were always darker than those evoked by ipsilateral (ipsi) eye stimulation. The average ODI was always positive and warm, red colors prevailed in the 2-dimensional OD-map, indicating contralateral dominance and no ocular dominance plasticity.

Quantification of the data revealed that already in the late CP (P $28-34)$ and even after voluntary physical exercise, OD-plasticity was completely abolished in PSD-93 KO mice (ODI no MD: $0.32 \pm 0.04 ; n=3$; after 4d MD: $0.25 \pm 0.02 ; n=5 ; p=0.13$; t-test) (Figure 69 A). These results suggest that there is an earlier closure of the CP for OD-plasticity around P 28 if PSD- 
93 is absent. This cannot be preserved by running which was shown before to be effective in WT mice (Kalogeraki et al., 2014).

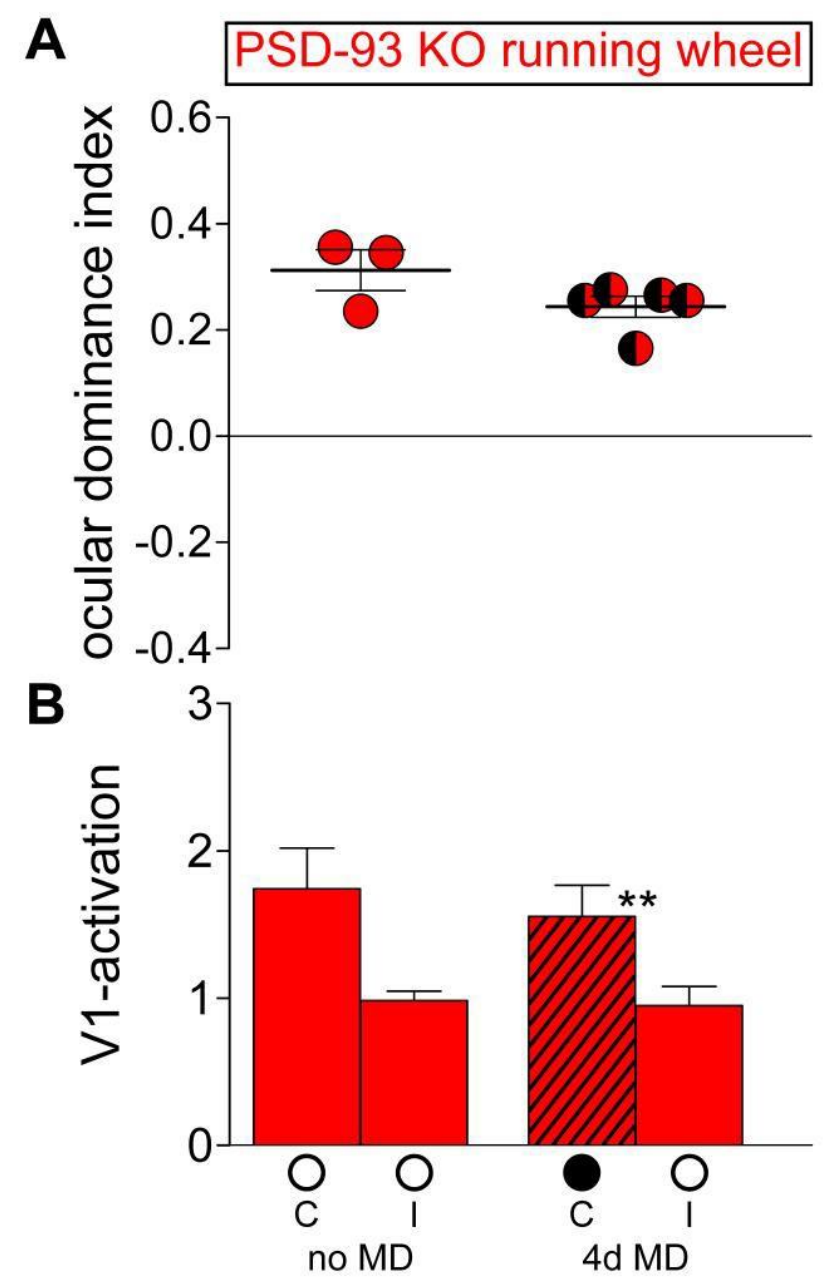

Figure 69: Ocular dominance indices and average response magnitudes of PSD-93 KO mice after physical exercise (running wheel) without MD $(n=3)$ and after four days of $M D(n=5)$ during the late-critical period.

(A) Positive ODI values indicate dominance of the contralateral eye, negative ODI values indicate ipsilateral dominance. Unicolored circles represent ODI values of individual animals without MD, half-filled circles represent individual animals after 4 days of $\mathrm{MD}$; means are marked by thick horizontal lines and the standard deviation is illustrated with small horizontal lines. PSD-93 KO mice without MD as well as mice after 4 days of MD showed ocular dominance of the contralateral eye and similar ODI values (t-test, $p=0.13$ ). (B) Average cortical responses expressed as a change in reflectance $\times 10^{-4}$ by stimulation of the contralateral (C) or ipsilateral (I) eye in PSD-93 KO mice without and with four days of MD. In all control animals without MD, cortical activation after visual stimulation of the contralateral eye was higher than after ipsilateral eye stimulation, reflecting the dominance of the contralateral eye in mice. After 4 days of MD, cortical responses did not change in PSD-93 KO mice and remained higher after contralateral eye stimulation in comparison to cortical activation after ipsilateral eye stimulation ( $p<0.01$; paired t-test). 


\subsection{Visual capabilities measured with the virtual-reality optomotor system in PSD-}

\section{$93 \mathrm{KO}$ mice after voluntary physical exercise in a running wheel}

\subsubsection{Maximum spatial frequency threshold (visual acuity)}

The virtual-reality optomotor system was used to determine the visual capabilities of PSD-93 KO mice after raising them in a standard cage with a running wheel allowing voluntary physical exercise. Mice were tested before and after four days of MD between P 24 - P 30. The maximum spatial frequency at which the optokinetic response (OKR) of the mice was still elicited was used to measure the visual acuity threshold of the optomotor reflex.

PSD-93 KO mice showed improved visual acuity over the time course of the 4-day MD-period from $0.391 \pm 0.002 \mathrm{cyc} / \mathrm{deg}$ on day 0 to $0.456 \pm 0.003 \mathrm{cyc} / \mathrm{deg}$ on day $4(\mathrm{n}=5)$ as shown in Figure $70 \mathrm{~A}$, corresponding to a total gain on baseline of $16.7 \pm 1.1 \%$ (Figure $70 \mathrm{~B}$ ). Mice without MD had visual acuity values of $0.390 \pm 0.004 \mathrm{cyc} / \mathrm{deg}(\mathrm{n}=3)$ which did not change over the testing period of 4 days (Figure $70 \mathrm{~A}$ ).

A

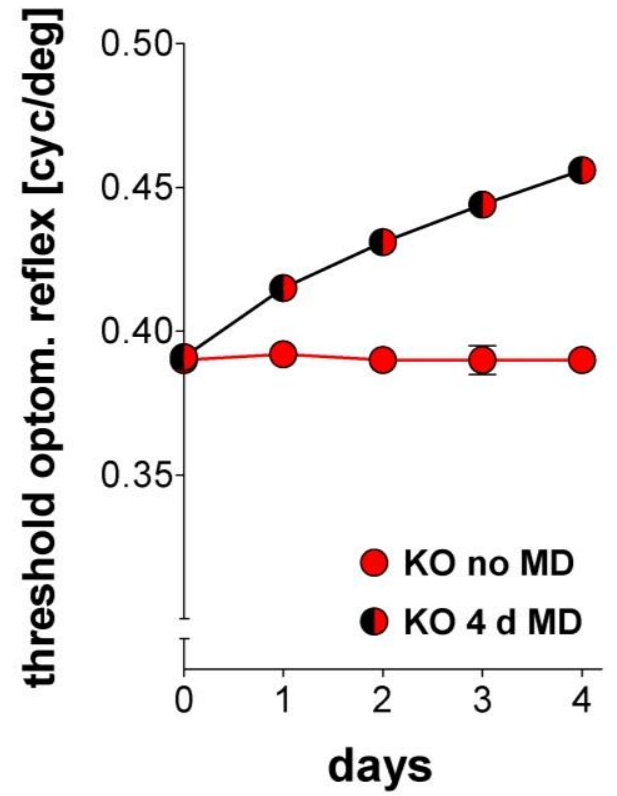

B

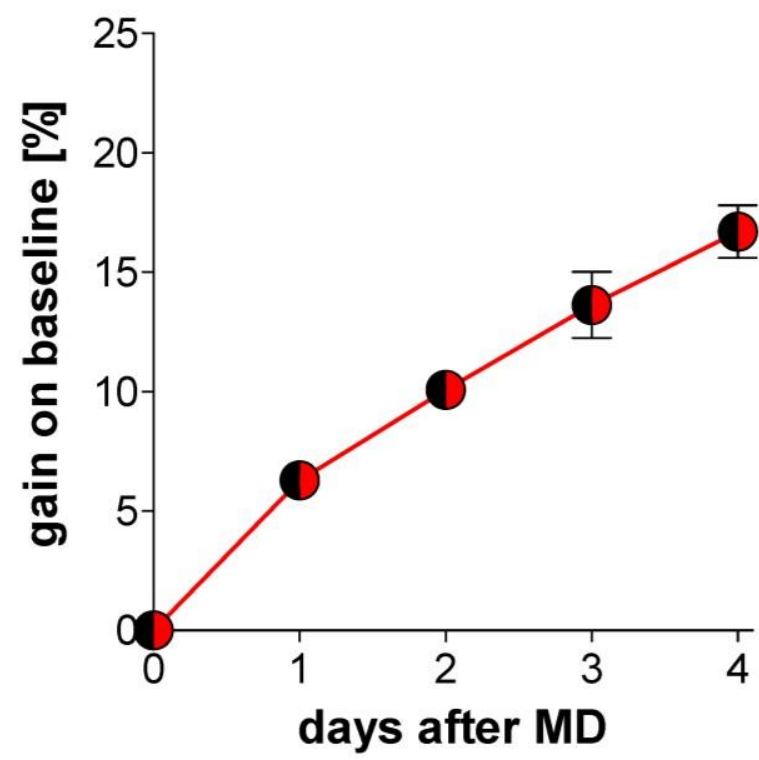

Figure 70: Time course of visual acuity and the gain on baseline during the late critical period of PSD-93 KO mice after voluntary physical exercise in a running wheel. Using the virtual-reality optomotor system, visual acuity (maximum perceived spatial frequency) of PSD-93 KO mice was determined. (A) The graph shows the enhancement of visual acuity after 4 days of MD (half-filled circles) in PSD-93 KO mice and of animals without MD (filled circles). The average values of visual acuity of all animals in one group are presented in cycles/degree on the y-axis with error bars (SEM). (B) Gain on baseline of visual acuity of PSD-93 KO mice after 4 days of MD. 
The average gain on baseline of all animals in one group is presented in per cent on the $y$-axis with respective error bars (standard deviation).

\subsubsection{Contrast sensitivity of PSD-93 KO mice during the late CP after voluntary physical exercise in a running wheel}

In addition to the maximum spatial frequency threshold of the optomotor reflex, the minimum contrast threshold at which the OKR was elicited was also determined using the optomotor system. Contrast sensitivity was determined at six different spatial frequencies: $0.031,0.064,0.092,0.103,0.192$, and $0.272 \mathrm{cyc} / \mathrm{deg}$ before and after 4 days of MD in PSD-93 KO mice. At each spatial frequency, the contrast was decreased stepwise until the OKR could not be observed anymore.

At a spatial frequency of $0.031 \mathrm{cyc} / \mathrm{deg}$, PSD-93 KO mice without MD showed a contrast sensitivity of $4.5 \pm 0.22$, at $0.064 \mathrm{cyc} / \mathrm{deg} 14.8 \pm 0.99$, at $0.092 \mathrm{cyc} / \mathrm{deg} 14.4 \pm 0.92$, at 0.103 cyc/deg $14.2 \pm 0.93$, at $0.192 \mathrm{cyc} / \mathrm{deg} 9.3 \pm 0.4$ and at $0.272 \mathrm{cyc} / \mathrm{deg} 4.5 \pm 0.23$ (Figure $71 \mathrm{~A}$ ). At a spatial frequency of $0.031 \mathrm{cyc} / \mathrm{deg}, \mathrm{PSD}-93 \mathrm{KO}$ mice showed contrast sensitivities of 4.4 \pm 0.12 which improved to $7.3 \pm 0.01$ after 4 days of $\mathrm{MD}$, at $0.064 \mathrm{cyc} / \mathrm{deg}$ it improved from $14.1 \pm 0.11$ to $20.6 \pm 0.64$, at $0.092 \mathrm{cyc} / \mathrm{deg}$ from $13.6 \pm 0.28$ to $20 \pm 0.86$, at $0.103 \mathrm{cyc} / \mathrm{deg}$ from $13.3 \pm 0.38$ to $19.4 \pm 0.9$, at $0.192 \mathrm{cyc} / \mathrm{deg}$ from $9.4 \pm 0.21$ to $13.4 \pm 0.38$ and at 0.272 cyc/deg from $4.5 \pm 0.1$ to $7.3 \pm 0.13$ (Figure $71 \mathrm{~B}$ ).

A

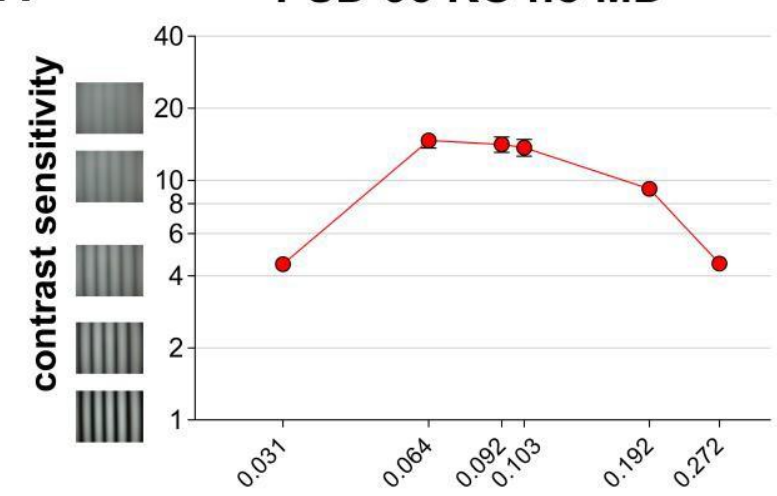

spatial frequency [cyc/deg]
B PSD-93 KO after 4 days of MD

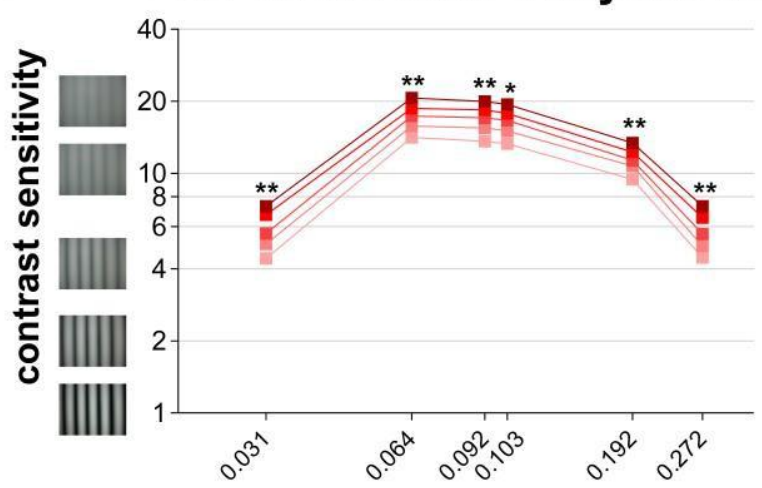

spatial frequency [cyc/deg]

Figure 71: Contrast sensitivity measured with the virtual-reality optomotor system (Prusky et al., 2004). Contrast sensitivity is plotted as a function of six different spatial frequencies in cycles per degree (cyc/deg). (A) Contrast sensitivity of PSD-93 KO mice without MD. (B) Enhancement of contrast sensitivity after 4 days of MD in PSD-93 KO mice during the late critical period. PSD-93 KO mice showed a significant improvement of contrast sensitivity after 4 days of MD ( $p<0.01$; t-test). 


\subsection{Visual cortex restricted knockdown of PSD-93 in WT mice}

Since it was shown that a visual cortex restricted knockdown of PSD-95 is sufficient to induce juvenile-like OD-plasticity in WT mice (Huang et al., 2015), it was investigated whether PSD93 manipulations selectively only the visual cortex are sufficient to induce an earlier closure of the CP for OD-plasticity in WT mice, as it was seen in PSD-93 KO mice before.

PSD-93 expression was silenced selectively only in the visual cortex of both hemispheres in WT mice (P 0 - P 2) with an adeno-associated virus (AAV) with a short hairpin (sh) RNA targeting PSD-93 (AAV - sh93). Control animals were injected with AAV sh - luciferase (AAV shlc). Animals were tested between P 28 - P 35 during the late CP after 4 days of MD with optical imaging of intrinsic signals in vivo.

Similar to WT and PSD-93 KO mice in the late CP, PSD-93 knockdown mice and controls without MD showed contralateral dominance with stronger visual cortical activation after contra eye stimulation than after visual stimulation of the ipsi eye. Representative 2dimensional ocular dominance maps in the left visual cortex of both experimental groups showed warm, red colors indicating contralateral dominance and all control mice exhibited positive ocular dominance indices (Figure 72). 


\section{WT AAV-shlc control no MD}

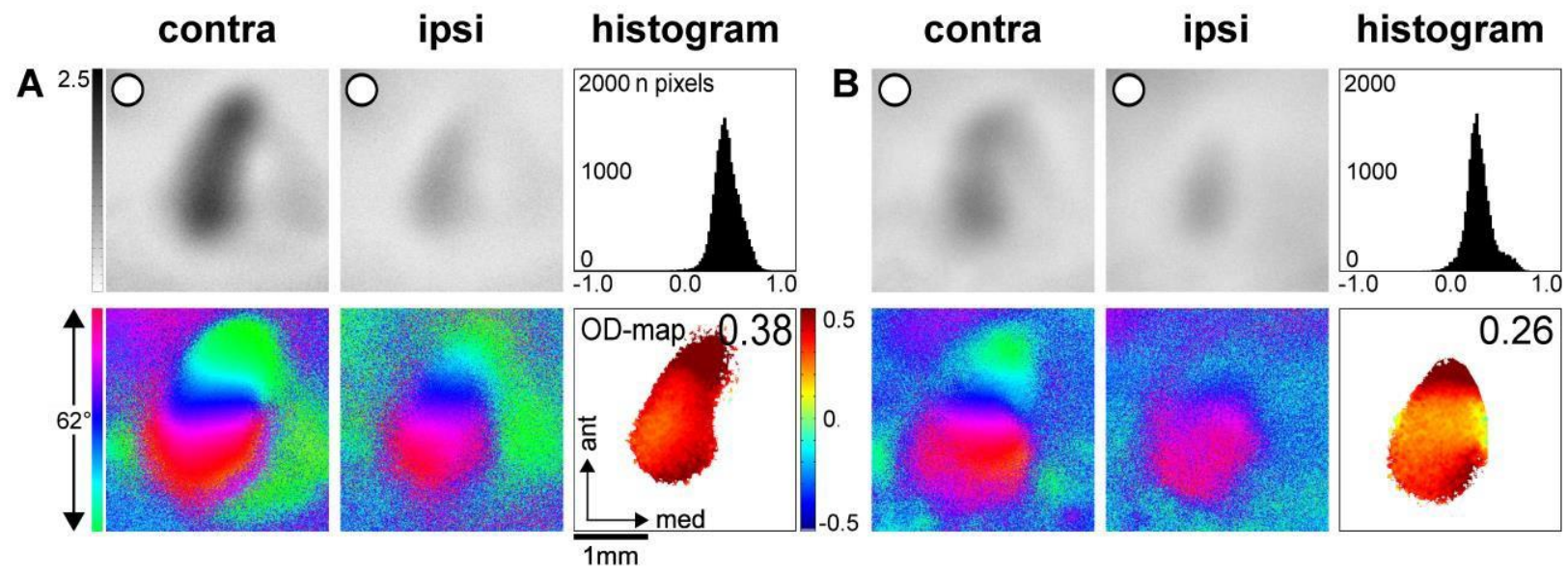

WT AAV-sh93 knockdown no MD
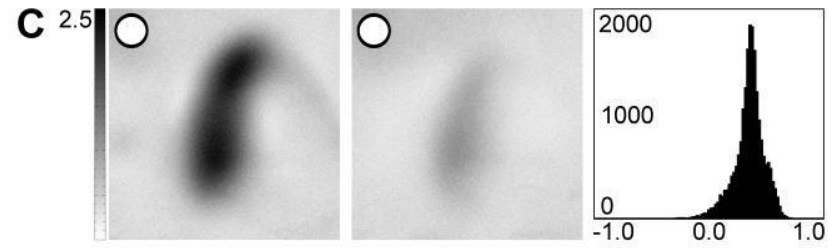

D 0
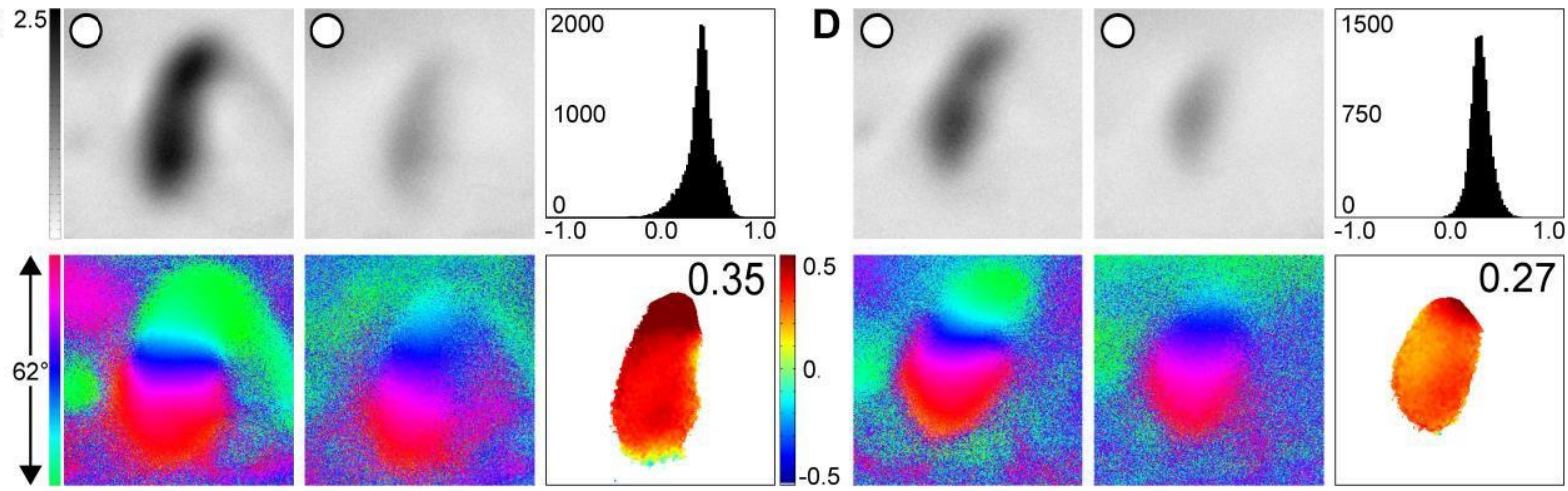

Figure 72: Representative examples of ocular dominance maps and indices in WT control mice without MD either injected with AAV - shlc (GFP controls) or AAV - sh93 (PSD-93 knockdown) in both visual cortices at P 0 -

P 2. Optical imaging maps of contralateral and ipsilateral cortical responses to visual stimulation of either eye with a horizontal moving bar (elevation) of $20^{\circ}$ are displayed from (A, B) two control mice and (C, D) two PSD93 knockdown mice without MD, respectively. Grayscale coded response magnitude maps (top rows, expressed as fractional change in reflectance $\times 10^{-4}$ ), color-coded phase maps of retinotopy (bottom rows), histograms of the OD-scores (top right of panels) and color-coded OD-maps (right bottom including the average ODI value), are illustrated. In both groups, the activity patches evoked by stimulation of the contralateral (contra) eye were always darker than those evoked by ipsilateral eye stimulation. The average ODI was positive and warm, red colors prevailed in the 2-dimensional OD-map, indicating contralateral dominance.

After 4 days of monocular deprivation, control injected mice showed OD-plasticity with weakened cortical activation after visual stimulation of the contra eye, whereas ipsi eye stimulation activated the visual cortex similarly strong as in mice without MD. The representative 2-dimensional ocular dominance maps in the left V1 showed cold, blue colors 
indicating ipsilateral dominance. The histograms were shifted to the left and all mice exhibited negative ocular dominance indices (Figure $73 \mathrm{~A}, \mathrm{~B}$ ).

In contrast to control injected mice, this was not the case in PSD-93 knockdown mice. Here, 4 days of MD could not induce OD-plasticity and the cortical activation after visual stimulation of the contra eye remained higher than after visual stimulation of the ipsi eye. Additionally, the 2-dimensional OD-map showed warm, red colors indicating contralateral dominance and all mice exhibited positive ocular dominance indices (Figure $73 \mathrm{C}, \mathrm{D}$ ).

\section{WT AAV-shlc control after 4 days of MD}

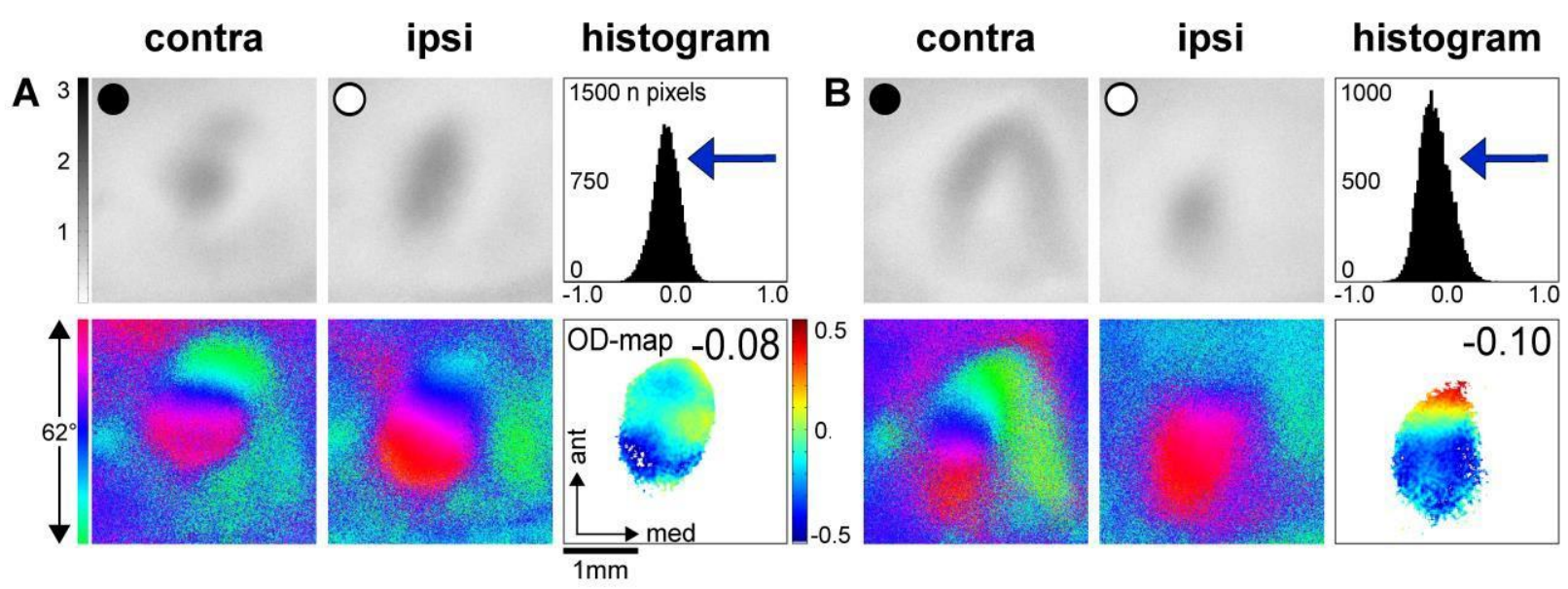

WT AAV-sh93 knockdown after 4 days of MD
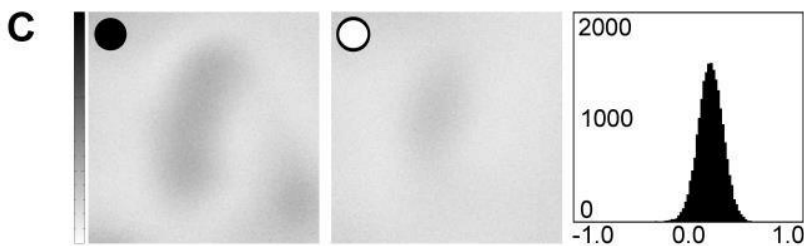

D
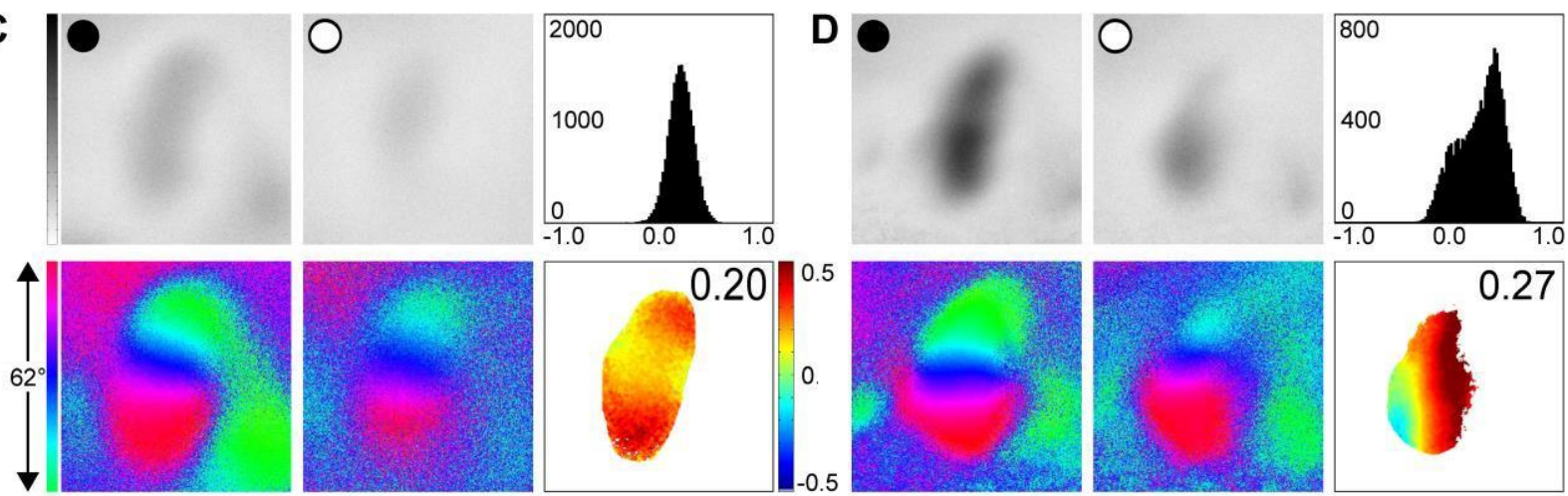

Figure 73: Representative examples of ocular dominance maps and indices in WT mice after four days of MD either injected with AAV - shlc (GFP controls) or AAV - sh93 (PSD-93 knockdown) in both visual cortices at P 0 P 2. Optical imaging maps of contralateral and ipsilateral cortical responses to visual stimulation of either eye with a horizontal moving bar (elevation) of $20^{\circ}$ are displayed from (A, B) two control mice and (C, D) two PSD93 knockdown mice after 4 days of $M D$, respectively. Grayscale coded response magnitude maps (top rows, expressed as fractional change in reflectance $\times 10^{-4}$ ) with black circles indicating monocular deprivation of the eye and white circles indicating that the respective eye remained open. Color-coded phase maps of retinotopy (bottom rows), histograms of the OD-scores (top right of panels) and color-coded OD-maps (right bottom including the average ODI value), are illustrated. After 4 days of MD in WT control injected mice, there was an 
OD-shift towards zero. Activity patches evoked by stimulation of the contralateral eye were equally strong to that after ipsi eye stimulation and the histogram shifted to the left. Average ODI were negative and cold, blue colors prevailed in the 2-dimensional OD-map, indicating ipsilateral dominance and hence ocular dominance plasticity. In contrast to that, in PSD-93 knockdown mice, the activity patches evoked by stimulation of the contralateral eye after MD were still darker than those evoked after ipsilateral eye stimulation. The average ODI was always positive and warm, red colors prevailed in the 2-dimensional OD-map, indicating contralateral dominance and no ocular dominance plasticity.

In mice with a visual cortex specific knockdown of PSD-93, 4 days of MD could not induce an OD-shift (ODI: $0.20 \pm 0.02 ; n=6$ ), whereas in age-matched control mice injected with AAV shlc, OD-plasticity was present after 4 days of MD (ODI: $-0.07 \pm 0.02 ; n=3, p<0.001$; t-test) (Figure $74 \mathrm{~A}$ ). This strong OD-shift in control injected WT mice was mediated by a reduction of deprived eye responses (WT shlc late CP contra no MD: $1.88 \pm 0.06 ; n=3$; WT shlc late $C P$ contra 4d MD: $0.81 \pm 0.08 ; n=3 ; p<0.001$; t-test), whereas ipsilateral eye evoked responses in V1 remained unchanged (WT shlc late CP ipsi no MD: $1.01 \pm 0.01 ; n=3$; WT shlc late CP ipsi 4d MD: $0.93 \pm 0.12 ; p=0.6$; t-test) (Figure 74 B). In PSD-93 knockdown mice, the visual cortex was still dominated by cortical activation of the contralateral eye after 4 days of MD (WT sh93 late CP contra no MD: $2.0 \pm 0.2 ; n=3$; WT sh93 late CP contra 4d MD: $1.4 \pm 0.2 ; n=6 ; p=0.1$; t-test). Furthermore, the ipsilateral eye evoked responses in V1 did not change after MD in PSD-93 knockdown mice (WT sh93 late CP ipsi no MD: $1.13 \pm 0.02 ; n=3$; WT sh93 late CP ipsi 4d MD: $0.91 \pm 0.12 ; n=6 ; p=0.26$; t-test) as shown in Figure $74 \mathrm{~B}$. 
A
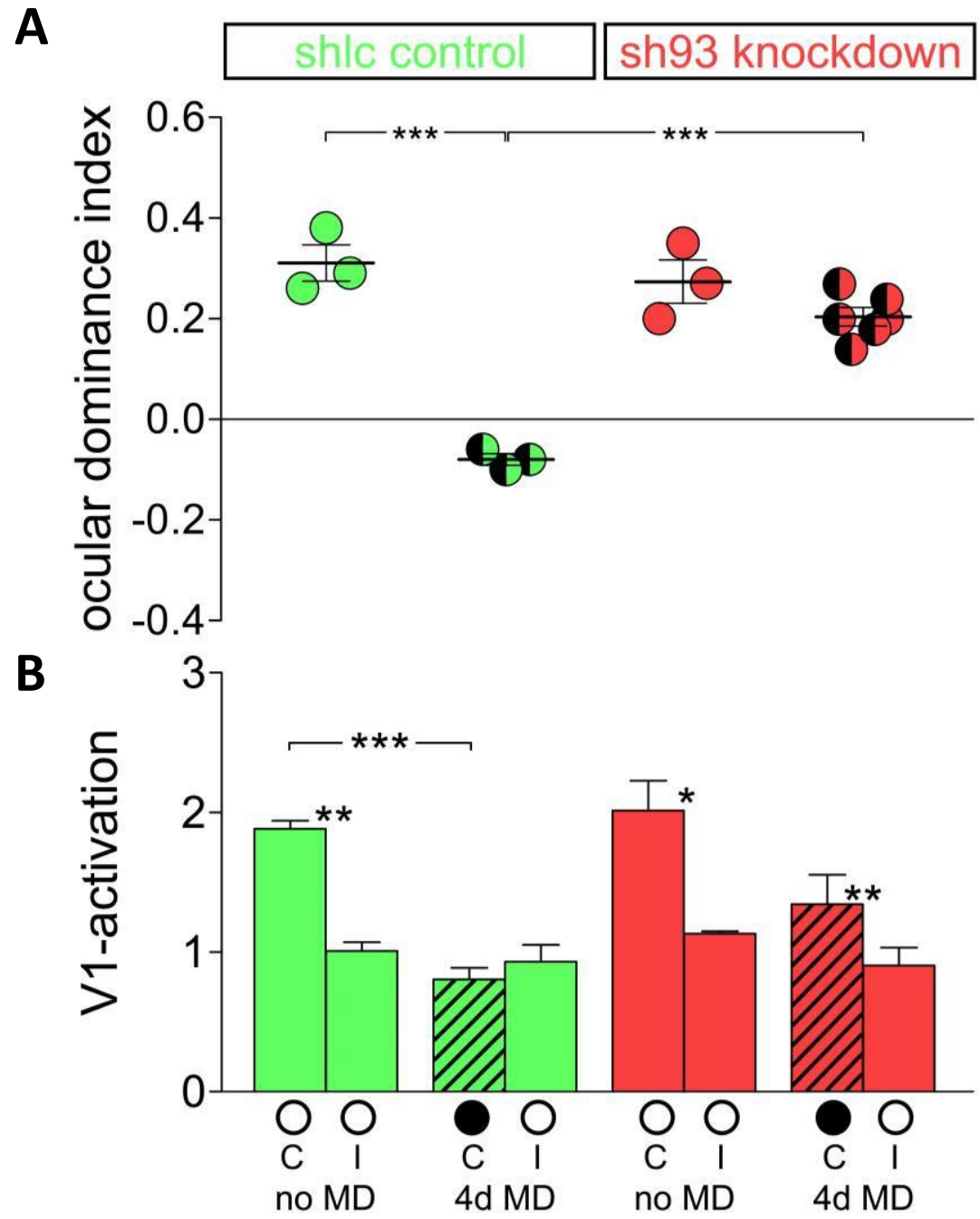

Figure 74: Ocular dominance indices and average response magnitudes of WT mice injected with a control virus (AAV - shlc; $n=3$ ) or with a knockdown of PSD-93 (AAV - sh93; $n=3$ ) without MD and after 4 days of MD (control, $\mathrm{n}=3$ / PSD-93 KD, $\mathrm{n}=6$ ) during the late CP. (A) Positive ODI values indicate dominance of the contralateral eye, negative ODI values represent ipsilateral dominance. Unicolored circles represent ODI values of individual animals without MD, half-filled circles represent individual animals after 4 days of MD; means are marked by thick horizontal lines and the standard deviation is illustrated with small horizontal lines. WT control injected mice (green) and PSD-93 knockdown mice (red) without MD showed ocular dominance of the contralateral eye and similar ODI values (t-test, $p=0.55$ ). Four days of monocular deprivation in control mice induced a significant OD-shift towards the open eye (t-test, $p<0.001$ ), which was significantly different from values in PSD-93 knockdown mice, which did not show ocular dominance plasticity after MD (t-test, $p<0.001)$. (B) Average cortical responses expressed as a change in reflectance $\times 10^{-4}$ by stimulation of the contralateral (C) or ipsilateral (I) eye in control and PSD-93 knockdown mice without MD and after four days of MD. In all animals without MD, cortical activation after visual stimulation of the contralateral eye was significantly higher than after ipsilateral eye stimulation (control: $p<0.01$; PSD-93 KD: $p<0.05$; paired t-test), reflecting the dominance of the contralateral eye in mice. In control mice after four days of $M D$, the response strength of the two eyes were no longer significantly different. The deprived eye responses were significantly reduced compared to that 
of mice without MD ( $p<0.001$; t-test). In contrast, cortical responses did not change in PSD-93 knockdown mice after MD and remained higher after contralateral eye stimulation in comparison to cortical activation after ipsilateral eye stimulation ( $p<0.01$; paired t-test).

This result is consistent with the previous observation that 4 days of MD are not sufficient to induce OD-plasticity in PSD-93 deficient mice in the visual cortex beyond P 28. Control injected animals showed the same phenotype as WT mice with a strong OD-shift after 4 days of MD, which was also mediated by a depression of deprived eye responses in V1.

\subsection{Visual capabilities measured with the virtual-reality optomotor system in WT mice after a visual cortex restricted knockdown of PSD-93}

\subsubsection{Maximum spatial frequency threshold (visual acuity)}

The virtual-reality optomotor system (OPT; Prusky et al., 2004) was used to determine visual capabilities of WT mice after a visual cortex restricted virus injection of either AAV - shlc (control; $n=3$ ) or after a knockdown of PSD-93 (AAV - sh93; $n=6$ ) between P 28 - 35. The maximum spatial frequency at which the optokinetic response (OKR) of the mice was still elicited, was used to measure the visual acuity threshold of the optomotor reflex (from now referred to as 'visual acuity').

In control mice without MD, visual acuity was not significantly different from mice with a visual cortex restricted knockdown of PSD-93 (AAV - shlc: $0.391 \pm 0.002$ cyc/deg; $n=3$; AAV sh93: $0.392 \pm 0.004 ; n=3 ; p=0.5 ;$ t-test).

It was examined whether control injected and PSD-93 KD mice show a similar visual acuity improvement over the time period of 4 days after MD. PSD-93 KD mice improved from 0.390 $\pm 0.001 \mathrm{cyc} / \mathrm{deg}$ on day 0 to $0.454 \pm 0.005 \mathrm{cyc} / \mathrm{deg}$ on day $4(\mathrm{n}=6)$. Control mice improved from $0.388 \pm 0.004 \mathrm{cyc} / \mathrm{deg}$ on day 0 to $0.451 \pm 0.005 \mathrm{cyc} / \mathrm{deg}$ on day $4(\mathrm{n}=3)$. Both groups had similar visual acuity improvement ( $p<0.001$; repeated measures ANOVA) and did not differ from each other ( $p>0.05$, repeated measure ANOVA with Bonferroni correction) (Figure 75 A). The visual acuity increase corresponded to a total gain on baseline of $17 \pm 1.9 \%$ in controls and $17 \pm 1.2 \%$ in PSD-93 KD mice ( $p=0.91$; t-test). 
A

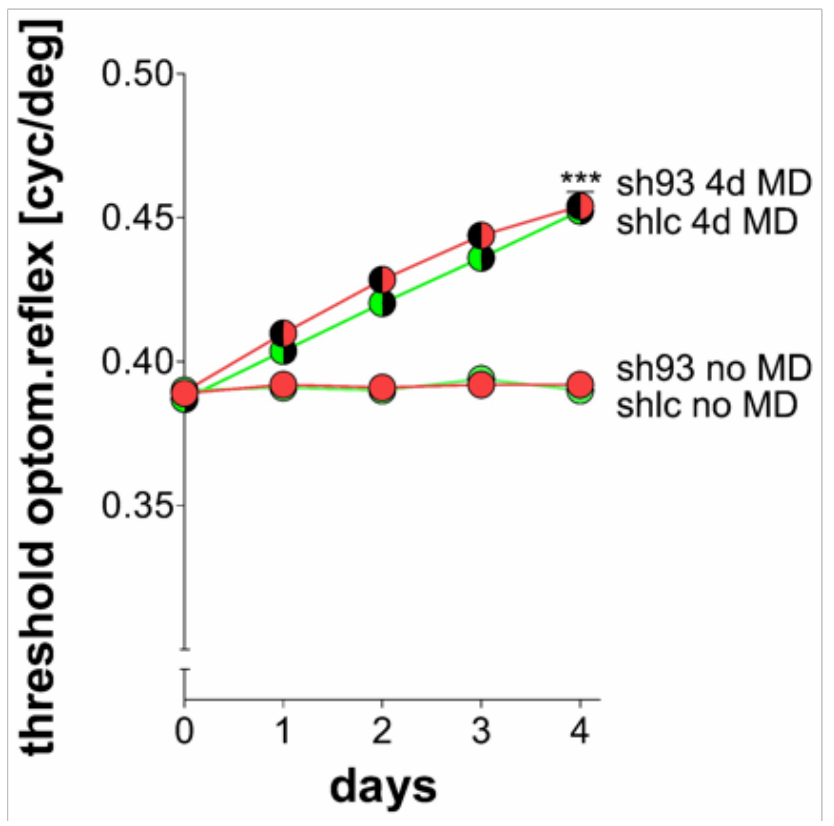

B

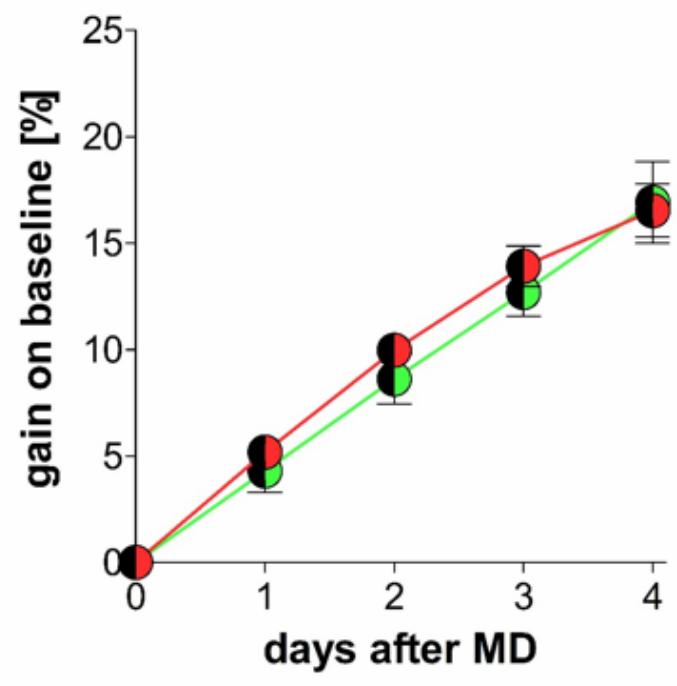

Figure 75: Time course of visual acuity and gain on baseline of WT control mice (AAV - shlc) and PSD-93 KD (AAV - sh93) mice. Using the virtual-reality optomotor system, visual acuity (maximum perceived spatial frequency) of PSD-93 KD (red) and control (green) mice was determined. (A) The graph shows the enhancement of visual acuity after 4 days of MD (half-filled circles) in control (green) and PSD-93 KD (red) mice and of animals without MD (filled circles). The average values of visual acuity of all animals in one group are presented in cycles/degree on the y-axis with error bars (SEM). (B) The graph shows the gain on baseline of visual acuity in control (green) and PSD-93 KD (red) mice over the time course of 4 days of MD. The average gain on baseline of all animals in one group is presented in per cent on the $y$-axis with respective error bars (standard deviation). Both groups did not differ from each other in the gain on baseline (t-test, $p=0.91$, t-test).

The data indicate that visual acuity is not compromised after a knockdown of PSD-93 in the visual cortex and like that of control injected WT mice, with both groups showing improved visual acuity of the open eye after 4 days of MD over the time course of the 4-day MD-period.

\subsubsection{Minimum contrast threshold (contrast sensitivity)}

WT control injected (AAV - shlc) and PSD-93 KD (AAV - sh93) mice were also tested for the minimum contrast threshold of the non-deprived eye which was measured with the virtualreality optomotor system after 4 days of MD daily. Contrast sensitivities were measured at six different spatial frequencies. On each day following monocular deprivation, the contrast sensitivity of all measured spatial frequencies increased substantially in both groups. 
Contrast sensitivity in control mice after 4 days of MD increased from day 0 to day 4 from 4.1 \pm 0.15 to $5.7 \pm 0.1$ at a spatial frequency of $0.031 \mathrm{cyc} / \mathrm{deg}$, from $14.1 \pm 0.2$ to $21.73 \pm 0.94$ at $0.064 \mathrm{cyc} / \mathrm{deg}$, from $13.3 \pm 0.05$ to $20.3 \pm 1.1$ at $0.092 \mathrm{cyc} / \mathrm{deg}$, from $12.9 \pm 0.1$ to $19.8 \pm 1.1$ at $0.103 \mathrm{cyc} / \mathrm{deg}$, from $8.8 \pm 0.15$ to $14.38 \pm 0.6$ at $0.192 \mathrm{cyc} / \mathrm{deg}$, from $4.03 \pm 0.14$ to $5.7 \pm$ 0.11 at $0.272 \mathrm{cyc} / \mathrm{deg}$ with a peak of contrast sensitivity at $0.064 \mathrm{cyc} / \mathrm{deg}$ (Figure $76 \mathrm{~A}$ ).

Likewise, contrast sensitivity of PSD-93 KD mice after 4 days of MD increased from day 0 to day 4 from $4.26 \pm 0.01$ to $5.43 \pm 0.12$ at a spatial frequency of $0.031 \mathrm{cyc} / \mathrm{deg}$, from $13.64 \pm$ 0.25 to $21.35 \pm 1.0$ at $0.064 \mathrm{cyc} / \mathrm{deg}$, from $13.24 \pm 0.18$ to $20.33 \pm 0.74$ at $0.092 \mathrm{cyc} / \mathrm{deg}$, from $13 \pm 0.17$ to $20.1 \pm 0.9$ at $0.103 \mathrm{cyc} / \mathrm{deg}$, from $9.2 \pm 0.4$ to $13.6 \pm 0.4$ at $0.192 \mathrm{cyc} / \mathrm{deg}$, from $4.27 \pm 0.02$ to $5.5 \pm 0.01$ at $0.272 \mathrm{cyc} / \mathrm{deg}$ (Figure $76 \mathrm{~B}$ ).

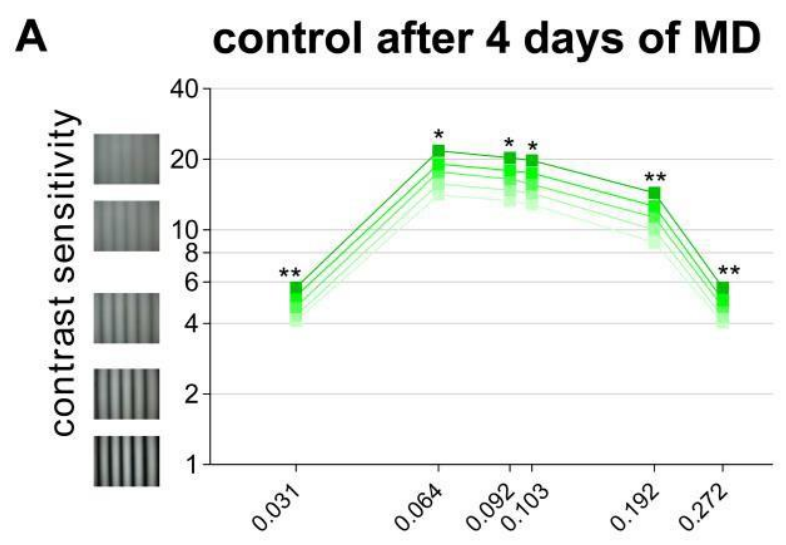

spatial frequency [cyc/deg]

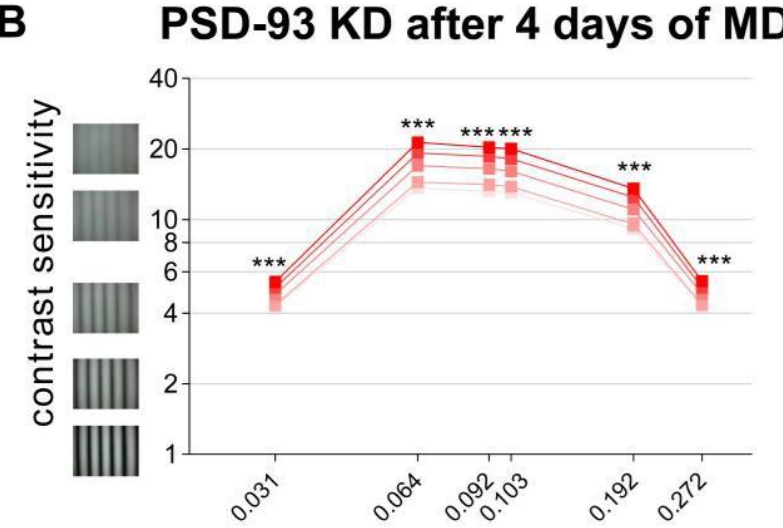

spatial frequency [cyc/deg]

Figure 76: Enhancement of contrast sensitivity of (A) AAV - shlc (control) and (B) AAV - sh93 (PSD-93 KD) mice during the late critical period after 4 days of monocular deprivation measured with the virtual-reality optomotor system (Prusky et al., 2004). Contrast sensitivity is plotted as a function of six different spatial frequencies in cycles per degree (cyc/deg). Both controls (green) and PSD-93 KD (red) mice showed a significant improvement of contrast sensitivity after 4 days of $M D(p<0.05$; t-test) and genotype did not have an influence on the improvement of contrast sensitivity after MD ( $p>0.05$; ANOVA).

The knockdown of PSD-93 in the visual cortex of WT mice at P $0-P 2$ did neither had an effect on contrast sensitivity ( $p>0.05$; ANOVA) nor on the improvement of it after four days of MD $(p>0.05 ;$ ANOVA). 


\subsection{Retinotopy and map quality of PSD-93 knockout and WT mice}

To test whether the visual cortex of PSD-93 KO mice is similarly organized to that of WT mice, V1 topographic maps measured with optical imaging of intrinsic signals were analyzed. Therefore, the contralateral eye was stimulated with either horizontally or vertically moving bars over the full monitor screen (Figure $77 \mathrm{~A}, \mathrm{D}$ ).

V1-activation (elevation: control: $1.22 \pm 0.21 ; n=9 ; \mathrm{KO}: 3.11 \pm 0.221 ; n=9 ; p=0.993$; azimuth: WT: $2.29 \pm 0.145 ; n=9 ; K O: 2.03 \pm 0.144 ; n=9 ; p=0.22$ ) and retinotopic map quality (map scatter) (elevation: WT: $1.06 \pm 0.275 ; n=9 ;$ KO: $0.87 \pm 0.158 ; n=9 ; p=0.573$; azimuth: WT: $2.45 \pm 0.286 ; n=9 ; K O: 3.21 \pm 0.515 ; n=9 ; p=0.197$ ) were similar in PSD-93 KO and WT mice (Figure 77).
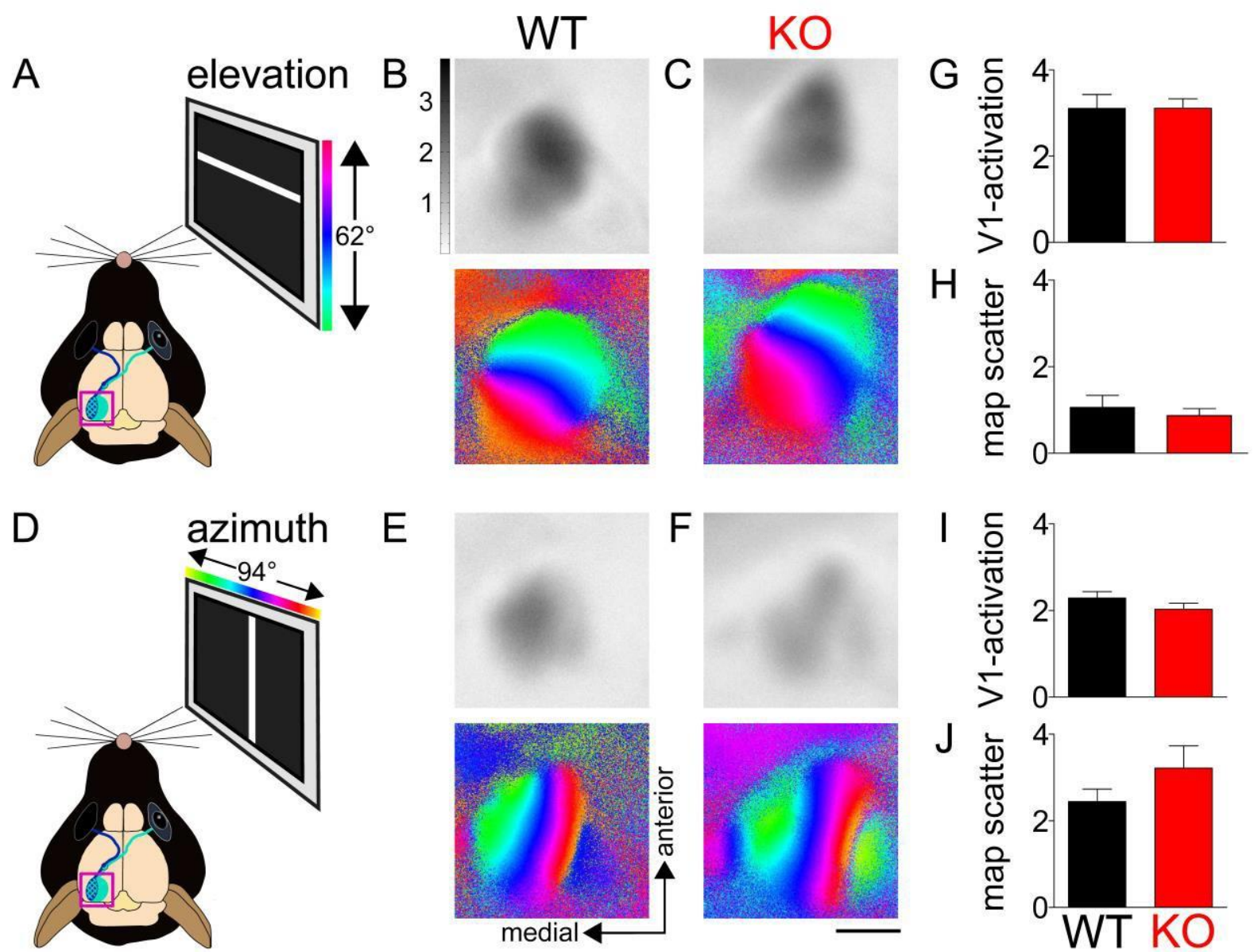

Figure 77: Representative examples of activity and retinotopic maps after elevation and azimuth stimulation recorded from WT and PSD-93 KO mice. There were no differences in the activity or quality of the maps between the different genotypes for both, (A) elevation stimulation in (B) WT mice or (C) PSD-93 KO mice or for (D) azimuth stimulation in (E) WT mice or (F) PSD-93 KO mice. Comparison of (G, I) V1-activation and (H, J) map quality after right eye stimulation between WT (black) and PSD-93 KO (red) mice revealed no significant 
differences between the genotypes. Acquired maps were identical without significant differences in either signal strength or quality of retinotopy for both elevation (V1-activation: $p=0.993$; map scatter: $p=0.573$; t-test) and azimuth (V1-activation: $p=0.22$; map scatter: $p=0.197$; t-test) stimulation. Scale bar: $1 \mathrm{~mm}$.

Overall low and similar map scatter values of both genotypes indicate good map quality which is not compromised in PSD-93 deficient mice. This was confirmed by the obtained elevation and azimuth maps with clear retinotopy and smooth transitions between the colors coding for retinotopy that were prevailing in both genotypes.

\subsection{Retinotopy and map quality of PSD-93 knockdown and control mice}

To test whether virus injections in the visual cortex of newborn pups did influence or effect cortical functions, V1 topographic maps measured with optical imaging of intrinsic signals in PSD-93 knockdown mice (AAV - sh93) or control injected mice (AAV - shlc) were also analyzed).

Therefore, the respective contralateral eye was stimulated with either horizontally or vertically moving bars as in the PSD-93 KO mice before. V1-activation (elevation: control: 3.14 $\pm 0.28 ; n=3 ; K D: 2.83 \pm 0.33 n=3 ; p=0.72$; azimuth: control: $2.23 \pm 0.05 ; n=3 ; K D: 2.09 \pm$ $0.5 ; n=3 ; p=0.78$; t-test) and retinotopic map quality (map scatter) (elevation: control: 1.22 $\pm 0.4 ; n=3 ; K D: 1.31 \pm 0.5 ; n=3 ; p=0.9 ;$ azimuth: control: $6.29 \pm 1.68 ; n=3 ; K D: 5.03 \pm 2.83$; $\mathrm{n}=3 ; \mathrm{p}=0.72$; t-test) were similar in WT mice with virus injections in the V1 of both hemispheres (Figure 78). 

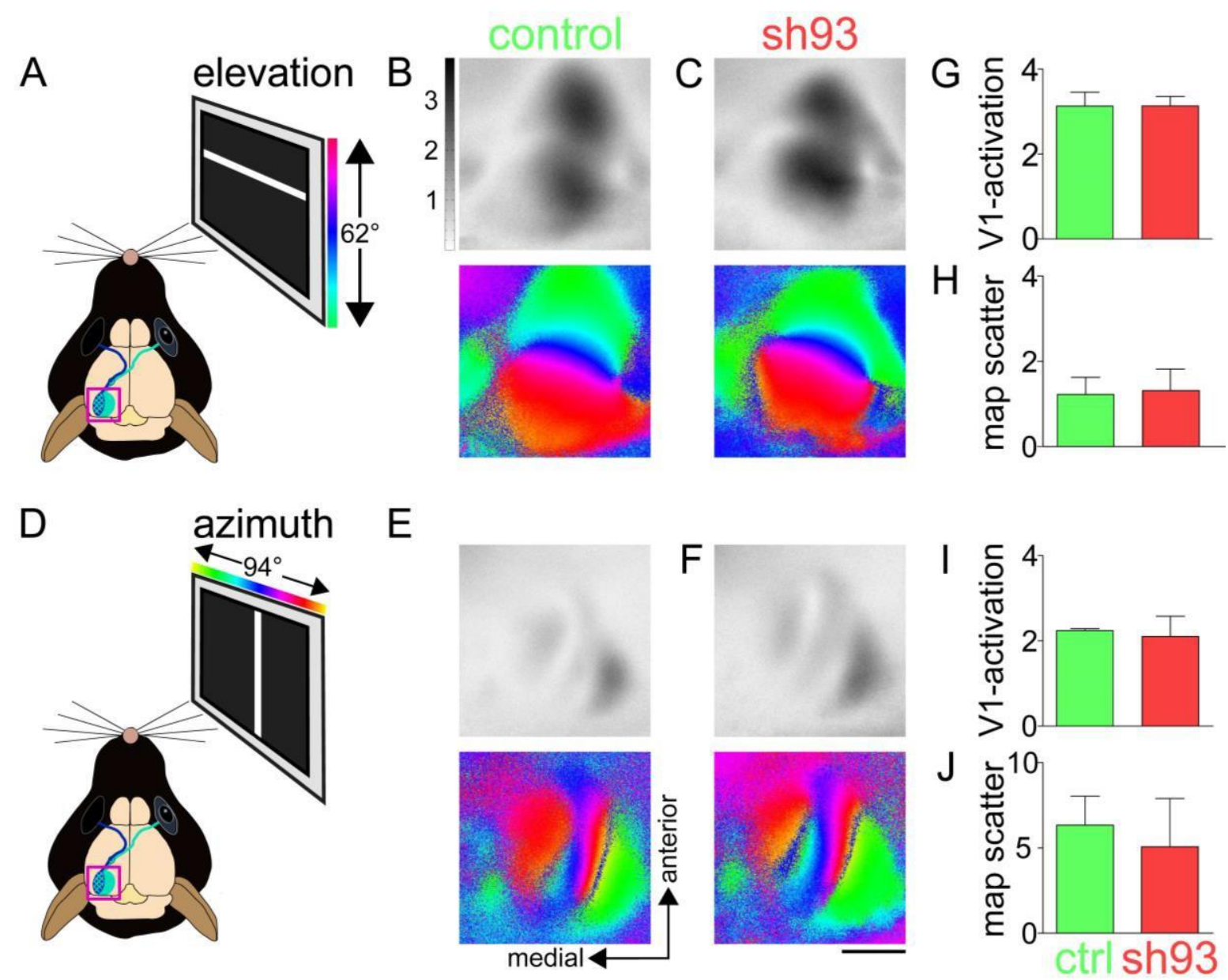

Figure 78: Representative examples of activity and retinotopic maps after elevation and azimuth stimulation recorded from WT mice either injected with a control virus (AAV - shlc) or with a knockdown of PSD-93 (AAV sh93). There were no differences in the activity or quality of the maps between the different groups. (A) elevation stimulation in (B) control mice or (C) PSD-93 knockdown mice or for (D) azimuth stimulation in (E) control- or (F) PSD-93 knockdown mice. Comparison of (G, I) V1-activation and (H, J) map quality after right eye stimulation between control (green) and PSD-93 knockdown (red) mice revealed no differences between the two groups with identical maps without significant differences in either signal strength or quality of retinotopy for both elevation (V1-activation: $p=0.72$; map scatter: $p=0.9$; $t$-test) and azimuth stimulation (V1-activation: $p=$ 0.78; map scatter: $\mathrm{p}=0.72$; t-test). Scale bar: $1 \mathrm{~mm}$.

\subsection{AAV transduction analysis of V1-specific PSD-93 knockdown and control mice} (P 0)

After optical imaging of intrinsic signals, animals were transcardially perfused and in situ brain pictures were taken using a Sony alpha 6000 camera and a BlueStar Nightsea flashlight with yellow filter glasses to excite GFP fluorescence in the specimen (Figure 79). 


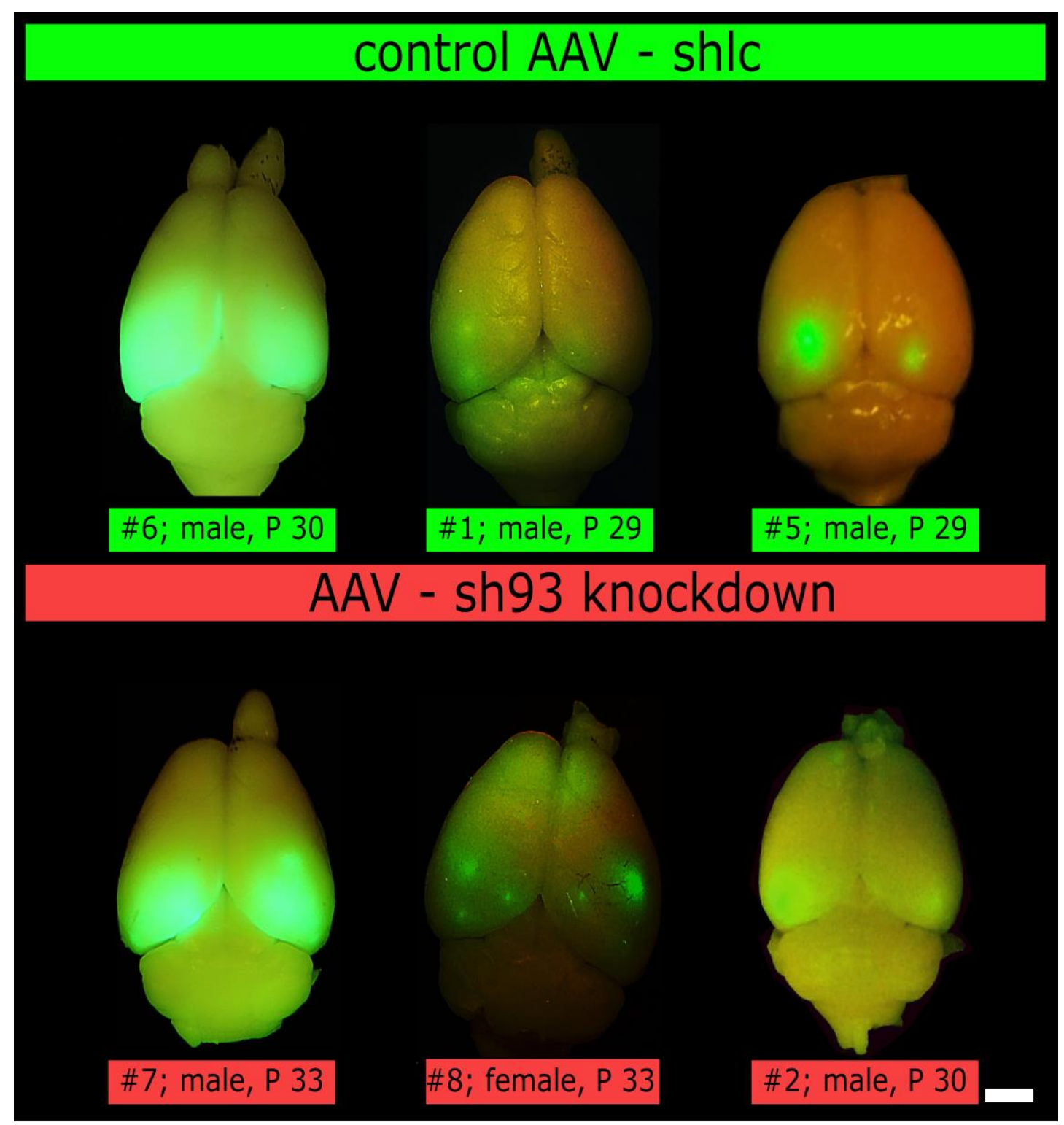

Figure 79: In situ brain images of virus injections of either AAV - shlc control or AAV - sh93 in P 0 WT mice. Brain images were taken from above to illustrate GFP fluorescence in control animals (AAV - shlc) and PSD-93 knockdown animals (AAV - sh93). Scale bar $1 \mathrm{~mm}$.

The brains were then sliced and mounted on glass slides. Confocal images were acquired with a Nikon SMZ18 microscope to analyze transfection efficiency of the control virus (AAV - shlc) or PSD-93 knockdown (AAV - sh93) mice (Figure 80).

The transfection efficiency in brain slices revealed no homogenous distribution of the virus in the visual cortex and variation between the different animals could be observed. However, it was effective to elicit the same phenotype regarding OD-plasticity as seen in ubiquitous PSD$93 \mathrm{KO}$ mice before. 


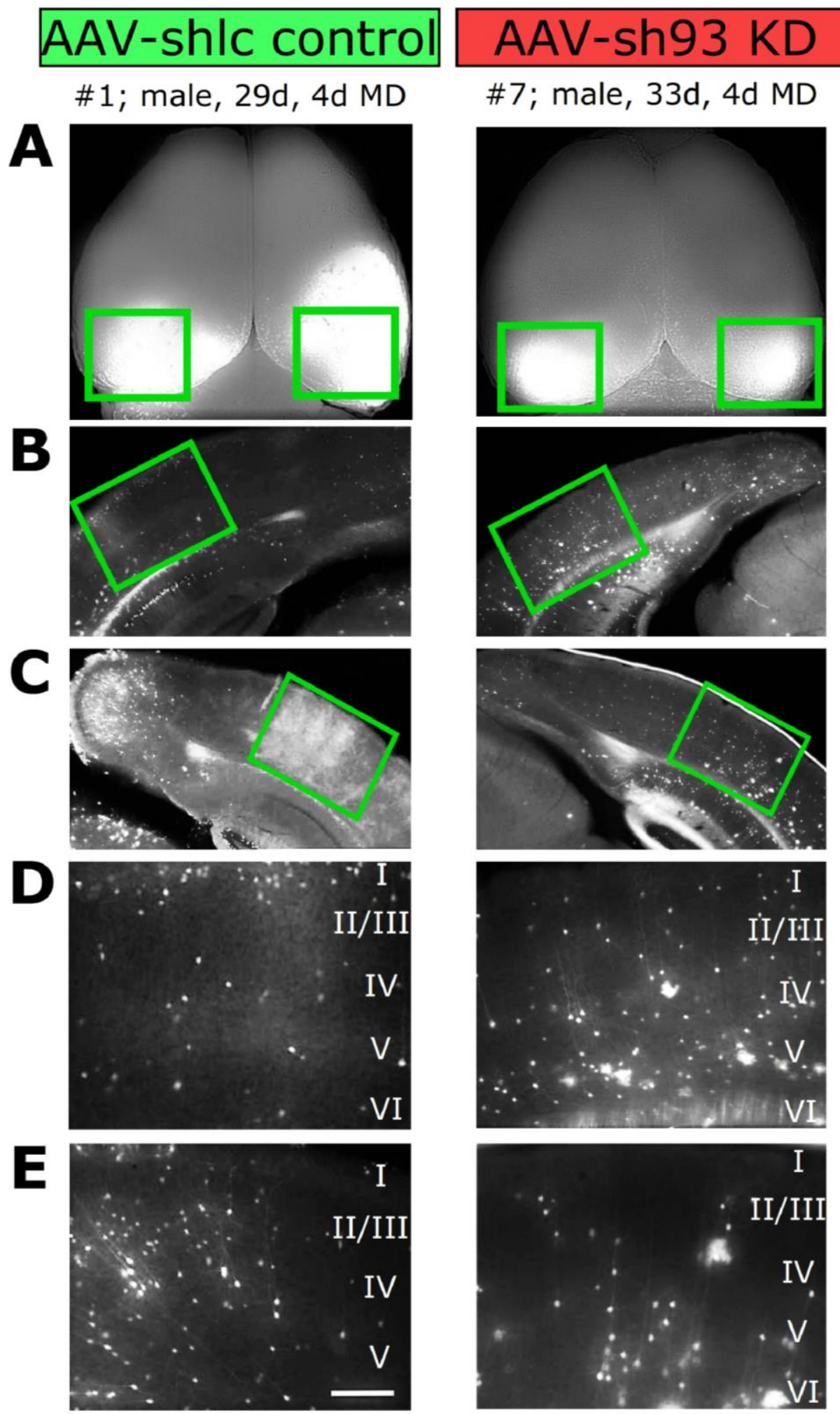

Figure 80: Microscopy images of virus transduction of either AAV - shlc control or AAV - sh93 WT mice injected at P 0. (A) Brain images taken from above to illustrate GFP fluorescence in control animals (AAV - shlc; animal \#1; male, 29 days old after 4 d MD) or PSD-93 knockdown animals (AAV - sh93; animal \#7; male, 33 days old after 4 d MD). (B) Images obtained from brain slices of the left hemisphere from either control animals (left column) or PSD-93 knockdown mice (right column) and (C) the respective right hemisphere. Green rectangles represent the area of the visual cortex of the respective hemisphere. (D) Images of the visual cortex of either control animals (left column) or PSD-93 knockdown animals (right column) of the left hemisphere or (E) the right hemisphere, respectively. Scale bar $200 \mu \mathrm{m}$. 
Transfection efficiency was determined by the level of fluorescence of the visual cortex from microscopy images obtained from all animals ( $A A V$ - shlc controls and AAV - sh93 knockdowns) and analyzed with the program ImageJ. Fluorescence intensity was determined by the mean gray value of each visual cortex that was subtracted from background noise. Analysis with Image J revealed no difference in the transfection efficiency of AAV - shlc control animals and AAV - sh93 knockdown mice (Figure 81).

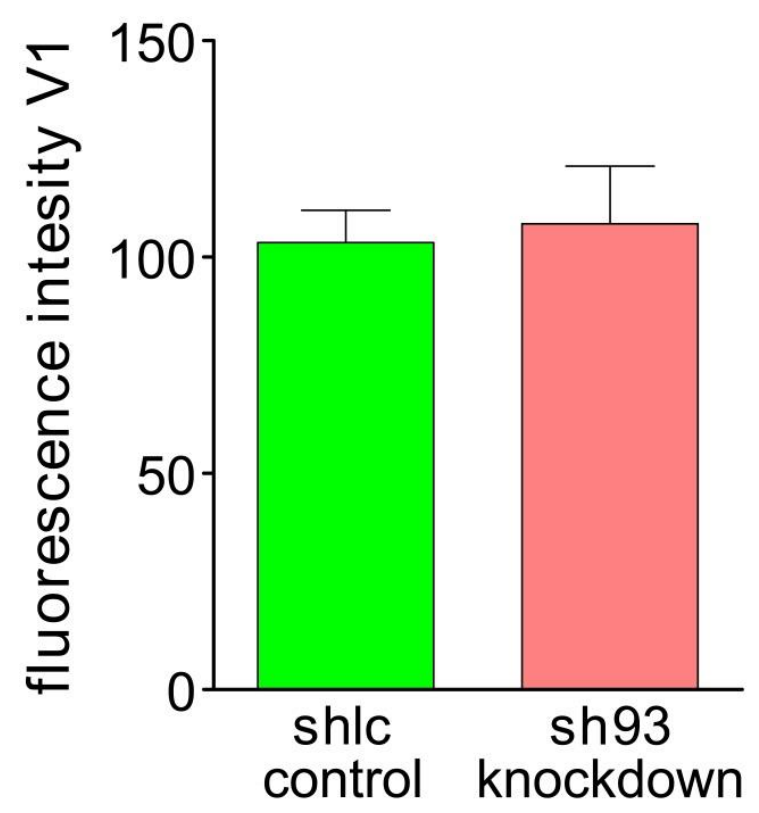

Figure 81: Fluorescence intensity measured in the visual cortex of AAV - shlc control and AAV - sh93 injected WT animals at $\mathbf{P} \mathbf{0}$. Fluorescence intensities in the visual cortex of control injected animals (AAV - shlc) were similar to that of PSD-93 knockdown animals (AAV - sh93) $(p>0.05)$.

There was also no statistical difference when comparing transfection efficiency in the visual cortex and obtained ocular dominance indices in the respective animals of either AAV - shlc control or AAV - sh93 knockdown animals (Figure 82). 


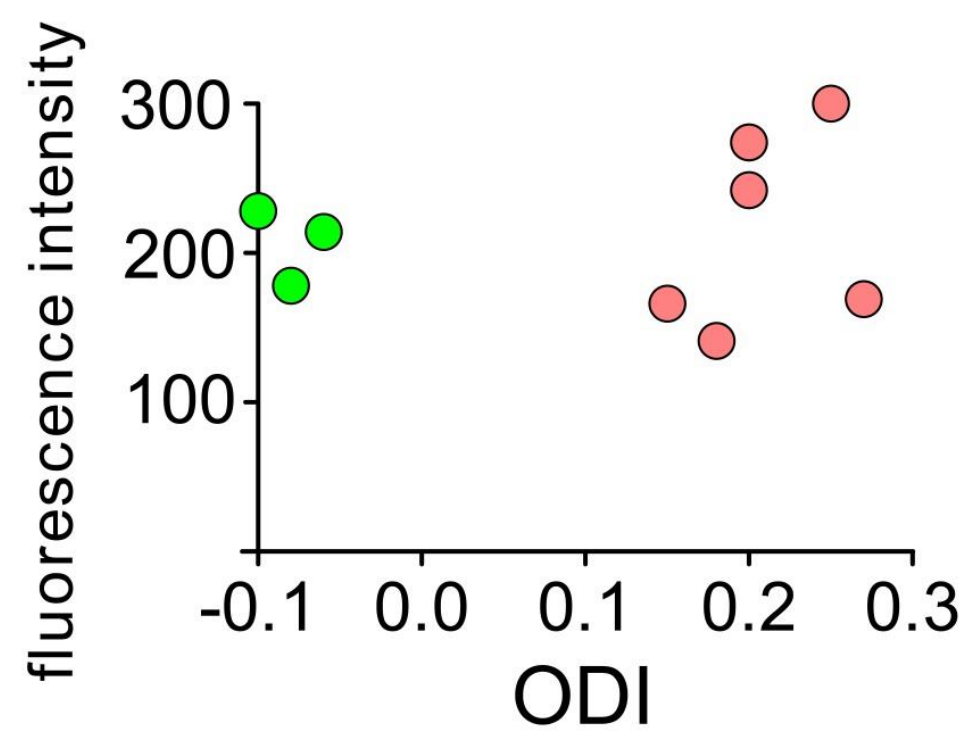

Figure 82: Fluorescence intensity measured in the visual cortex of AAV - shlc control (green symbols) and AAV sh93 (red symbols) injected WT animals at P 0 plotted against ocular dominance indices. Fluorescence intensities in the visual cortex of control injected animals (AAV - shlc) or PSD-93 knockdown animals (AAV - sh93) did not correlate with their respective ocular dominance indices. 


\section{Discussion}

This PhD project was aimed towards understanding the roles of the postsynaptic density (PSD) proteins PSD-95 and PSD-93 in cortical plasticity and vision in mice. Therefore, a variety of methods were combined, including optical imaging of intrinsic signals (Cang et al., 2005), behavioral assessments with the visual water task (Prusky et al., 2000) and the optomotor system (Prusky et al., 2004), viral knockdown (KD) of PSD-95 and PSD-93 in the visual cortex with adeno-associated viral vectors using shRNA and determination of transfection efficiency in the visual cortex were combined.

Firstly, ocular dominance (OD)-plasticity and interocular plasticity of PSD-95 and PSD-93 knockout (KO) mice and wildtype (WT) controls were assessed. Secondly, evaluation of whether a knockdown of either PSD-95 or PSD-93 only in the visual cortex (VC) affects the critical period (CP) for OD-plasticity was analyzed critically. Thirdly, the role of interhemispheric interactions for sensory processing and OD-plasticity in PSD-95 knockdown mice was determined. Finally, it was investigated whether both proteins play a role in visual capabilities of mice.

\subsection{PSD-95}

During early phases of life, mice and other mammals pass through critical periods of sensory system adjustments in order to adapt to the environment and achieve a high degree of functionality. To achieve optimal structural and functional properties, the brain has to undergo a transition from a highly plastic to a permanently consolidated state for stabilization of the acquired neuronal networks and skills.

In order to elucidate this developmental transition process, our work group proposes that AMPA receptor silent synapses serve as potent plasticity substrates for the activity- dependent strengthening of synaptic transmission at excitatory synapses in the visual cortex (Huang et al., 2015) as it was shown before in the hippocampus of mice (Isaac et al., 1995; Liao et al., 1995). These silent synapses are rather instable and require strong stimulation patterns, such as longterm potentiation (LTP), in order to be stabilized and then incorporated into a functional network (Isaac et al., 1995; Liao et al., 1995; Durand et al., 1996). During development and at the beginning of experience-dependent cortical network refinement, 
which is characterized by successive dendritic spine stabilization and decreased spine elimination (Zuo et al., 2005; Holtmaat et al., 2005), these AMPA receptor silent synapses are abundantly present. With early synaptic sensory pathway development, the number of silent synapses gradually decreases and matches the responsiveness for long-term potentiation (Rumpel et al., 1998). This cellular mechanism is thought to be controlled by experiencedependent strengthening and maturation of synapses (Ashby and Isaac, 2011).

The critical period is a maturational stage in the early lifespan of an animal, during which the nervous system is especially sensitive to certain environmental stimuli (Hensch, 2004). The general formation of synapses starts at birth and peaks before the critical period at around postnatal day (P) 16 in the rodent cortex (Blue and Parnavelas., 1983; Bourgeois, 1997). For mice it was also shown that synaptic connections between neurons in layer $2 / 3$ do not substantially increase further after the critical period (Funahashi et al., 2013). These observations suggest that synapses have been already established before the critical period and that their overall number does not change considerably afterwards. Instead, the functions and properties of already existing synapses are subjected to modification during further development.

Our collaborating partner, the work group under the direction of Prof. Dr. Dr. Schlüter (ENI, Göttingen), could recently show that normal WT mice exhibit about 50\% of AMPA receptor silent synapses in L4 to L2/3 synapses in the visual cortex during the time of eye opening when visual experiences start (Rumpel et al., 2004). However, this fraction of silent synapses then gradually declines up to early adulthood (P 60), where it comes to a standstill with less than $5 \%$ remaining silent synapses (Funahashi et al., 2013). Notably, in PSD-95 KO mice the opposite was observed: PSD-95 KO mice also exhibit about 50 \% of AMPA receptor silent synapses at eye opening, but this number remains constant throughout their whole lifespan (Huang et al., 2015) (Figure 20). A high fraction of AMPA receptor silent synapses can provide more possibilities for the conversion of immature synapses into stable and mature synapses. Our work group could successfully show that plasticity, including OD-plasticity, can be facilitated in mice when PSD-95 is lacking (Huang et al., 2015).

It is highly noteworthy that PSD-95 regulates both the gradual cortical development, including the maturation of AMPA receptor silent synapses, and the ending of the critical period for OD-plasticity (Huang et al., 2015). To emphasize this, it was shown that a deficiency of PSD95 directly leads to the absence of experience-dependent maturation of silent synapses and 
concurrently preserved a lifelong critical period-like OD-plasticity in PSD-95 KO mice (Figure 26). In various studies it has been reported multiple times that the number of silent synapses decreases during critical periods (Isaac et al., 1997; Funahashi et al., 2013). Notably, the observations made during this project show the reverse correlation: A lack of silent synapse maturation correlates with the persistence of juvenile-like OD-plasticity in the visual cortex of mice (Figure 26). Evidently, this indicates that prevention of silent synapse maturation leads to retention of the closure of the CP for juvenile-like OD-plasticity as well (Huang et al., 2015). Likewise, dark rearing (DR) from birth preserves juvenile-like OD-plasticity (Cynader et al., 1983; He et al., 2006) and also results in an elevated number of silent synapses (Funahashi et al., 2013). However, the closure of the critical period for OD-plasticity and the lack of silent synapse maturation have not been causally linked before. So far, several studies have suggested other cellular mechanisms to influence the properties of neural circuits by dark rearing, including the excitatory/inhibitory balance and metaplasticity (delaying the NMDA receptor subunit switch from GluN2B to GluN2A) (Guo et al., 2012; He et al., 2006; Morales et al., 2002; Philpot et al., 2003; Carmignoto and Vicini, 1992).

Based on previously described findings, it can be postulated that an impairment of the developmental switch of NMDA receptor subunits might not be the cause for the everlasting juvenile-like OD-plasticity in PSD-95 KO mice: Albeit, there are more NMDA receptors that contain synaptic GluN2B-subunits in young PSD-95 KO mice (Béique et al., 2006), the synaptic GluN2B levels are not changed in adult PSD-95 KO mice (Bonnet et al., 2013). Moreover, in mice with a knockout of GluN2A, OD-plasticity has been reported to be restricted rather than extended (Fagiolini et al., 2003).

Since the results of chapter 3.1. demonstrated lifelong juvenile-like OD-plasticity in ubiquitous PSD-95 KO mice, it was of high interest to narrow down the exact location of the OD-plasticity expression. Therefore, a visual cortex-restricted knockdown of PSD-95 in WT mice was conducted, which impaired excitatory synapse maturation (Huang et al., 2015) and also completely preserved juvenile-like OD-plasticity, extending into late adulthood (Figure 32). Furthermore, the site-specific depletion of PSD-95 from the visual cortex contralateral to the monocularly deprived eye was already sufficient to preserve juvenile-like OD-plasticity after only four days of monocular deprivation (Figure 32). These results helped to precisely identify the location of OD-plasticity expression in mouse visual cortex. In fact, it is rather restricted to the contralateral visual cortex of the deprived eye than a bilateral expression mechanism 
(Figure 32). Furthermore, the previously described conceptual model of PSD-95-dependent silent synapse maturation and hence the ending of the critical period for OD-plasticity was tested for applicability by knocking down PSD-95 in the visual cortex after the endpoint of the CP (P 40). Although at this point of time neural networks were already stabilized and the sensory system was fully functional, a knockdown of PSD-95 resulted in an unforeseen conversion of mature synapses to silent synapses. This observed phenomenon accurately supports our proposed conceptual model. Additionally, not only a rejuvenation of mature synapses but also a restoration of critical period-like OD-plasticity in WT mice at P 80 was observed (Figure 35). Moreover, with a similar approach, juvenile-like OD-plasticity could be restored in standard cage raised WT mice which were already mature and beyond the developmental phase in which OD-plasticity can still be induced (> P 110; Lehmann and Löwel, 2008). In detail, this was accomplished by knocking down PSD-95 in the visual cortex at approximately P 140. PSD-95 knockdown mice showed OD-plasticity after both four and seven days of MD (Figure 41). Hence, the described results highlight the decisive role of excitatory synapses of pyramidal neurons in regulating juvenile-like OD-plasticity in mice, which can be already effectively induced even in fully mature mice by knocking-down PSD-95.

To further strengthen our conceptual model; even a pharmacological manipulation of enhancing intracortical inhibition in vivo with Diazepam was unable to prevent juvenile-like OD-plasticity in PSD-95 KO mice. In contrast, WT mice treated with Diazepam failed to show OD-plasticity at all, which was completely blocked after monocular deprivation (Figure 26). Our collaborating group could further show that excitatory synapses onto PV positive interneurons as well as the inhibitory projection onto layer 2/3 pyramidal neurons were not altered in PSD-95 KO mice (Huang et al., 2015). This indicates that glutamatergic synapses onto PV positive interneurons do not require PSD-95 for their maturation. Consequently, the function of PSD-95 is not ubiquitous, but rather specific for the developmental unsilencing process of excitatory synapses onto pyramidal neurons. In addition to this, it does not alter intracortical inhibition itself.

It is noteworthy that the earlier postulated model challenges the current view, which states that increased intracortical inhibition is the cause for the closure of critical periods (Huang et al., 1999; Takesian and Hensch, 2013). Opposing this, the observations made during this project rather suggest that OD-plasticity is largely independent of the inhibitory tone and based on silent synapses on excitatory neurons. Importantly, the performed experiments 
(chapter 3.1. - 3.8.) could reveal that juvenile-like OD-plasticity in PSD-95 KO mice cannot only persist lifelong, but also be restored, even in the presence of high levels of intracortical inhibition. Likewise, if silent synapses are abundant by a knockdown of PSD-95, this allows restoration of OD-plasticity in WT mice which were already beyond the critical period (Huang et al., 2015) (Figure 35). Therefore, it can be proposed that the maturation of excitatory synapses onto V1 pyramidal neurons due to experience-dependent unsilencing of silent synapses leads to the closure of the critical period for juvenile-like OD-plasticity. Additionally, PSD-95 is absolutely required for the stabilization process of AMPA receptors in mature synapses. Essentially, the group under the direction of Prof. Dr. Dr. Schlüter found that $\sim 50$

$\%$ of all synapses matured independently of PSD-95 already before eye opening and therefore before the developmental increase of PSD-95 protein expression even starts (Yoshii et al., 2003) (Figure 20).

They drew the deduction that there might be at least two sub populations of synapses in the visual cortex: one sub population which is independent of PSD-95 and possibly more static and a second sub population that is plastic and dependent on a PSD-95 based experiencedependent maturation (Huang et al., 2015). This concept is supported by two indications: Firstly, a lack of PSD-95 after birth, during the development or after synapse maturation leads to both a reduction in AMPA receptor excitatory postsynaptic currents (EPSCs) and secondly, a reappearance of AMPA receptor silent synapses to an original level of approximately $50 \%$ (Huang et al., 2015). This falls in line with studies conducted in mouse or rat hippocampal CA1 pyramidal neurons which reported a reduction of approximately $50 \%$ in AMPA receptor EPSCs by PSD-95 loss-of-function approaches in still-developing synapses (Ehrlich et al., 2006; Béïque et al., 2006; Schlüter et al., 2006; Elias et al., 2006). Additionally, the absence of-PSD- 95 in excitatory synapses onto PV positive interneurons could not change basal functions of AMPA receptors (Huang et al., 2015). Notably, this remains to be observed when all the four MAGUK proteins are expressed in PV positive interneurons in the visual cortex (Akgul et al., 2010). Altogether, the described findings allow the postulation of a conceptual model that embraces both synapse stabilization during cortical network refinement and the closure of the critical period for OD-plasticity both caused by PSD-95. In these experience-dependent processes and network refinements, synapses will be either stabilized or pruned. The former was shown in 
the mouse barrel cortex (Ashby et al., 2011) and the later was reported in layer 5 pyramidal neurons (Zuo et al., 2005; Holtmaat et al., 2005).

However, one cannot rule out the important role of the inhibitory network in regulating critical period plasticity (Kuhlman et al., 2013; Morishita et al., 2008; Fagiolini and Hensch, 2000). It is very likely that not only PSD-95 alone governs silence synapse maturation and therefore regulates the critical period, but that it is rather a complex interplay with the inhibitory circuitry to refine cortical networks during the critical period in line with Hebbian plasticity (Smith et al., 2009; Hebb, 1949; Löwel and Singer, 1992). Importantly, the present data show that the inhibitory tone is permissive in regulating juvenile OD-plasticity, as its duration is governed by the presence of silent synapses and is independent of the inhibitory tone (Huang et al., 2015).

In particular, it is important to point out that juvenile OD-plasticity is not solely dependent on silent synapses, but can also be induced by other mechanisms under specific experimental conditions. For instance, multiple studies have shown that environmental enrichment or even simple physical activity alone cannot only prolong but also reinstate juvenile-like OD-plasticity (Greifzu et al., 2014; Kalogeraki et al., 2014). Importantly, this was likely independent of the generation of new silent synapses since the measured AMPA/NMDA receptor ratio was unchanged in the visual cortex of adult environmental enriched mice (Greifzu et al., 2014). There are at least two possibilities for the reinstated plasticity independent of the existence of silent synapses which are the following: First of all, there could be different expression mechanisms that cause the same system level read-out with depression of deprived eye responses after MD as a sign for juvenile-like plasticity. Secondly, a lowered degree of inhibition could also cause OD-plasticity in adult animals. Noteworthy, plasticity might not always be induced by the same mechanism throughout life but rather serves different purposes at different points of time.

For example, during the critical period synaptic connections are optimized and formed mainly by experience in early cortical development, whereas later in life, learning and plasticity is still possible but not as fast. It also potentially relies on other mechanisms to shape plasticity, such as changes in the inhibitory tone (Maya Vetencourt et al., 2008; Harauzov et al., 2010).

Silent synapses are ideally suited for the purpose of increased plasticity, since pre-existing synapses can be permanently integrated into the present network if they are repeatedly recruited by activity. However, if specific sensory features are not learned during the critical 
period and are accompanied by a general lack of activity, these synapses might be pruned. Consequently, an optimal development would be impossible to achieve (Espinosa and Stryker, 2012; Funahashi et al., 2013).

In previous studies, it was shown that a Nogo-66 receptor knockout results in a prolongation of OD-plasticity into adulthood after four days of MD, even until P 120 (McGee et al., 2005). The authors hypothesize that myelination is not the only factor, but one that is suppressing plasticity in the mature brain and is therefore strengthening neural circuits (McGee et al., 2005). Similarly, enhancement of OD-plasticity was reported in mice lacking the paired Ig-like receptor B (PirB). This was assessed by means of the activity-regulated immediate-early gene Arc mRNA induction and measured by intrinsic signal optical imaging (Syken et al., 2006; Djurisic et al., 2013). The Authors found increased OD-plasticity as well as $50 \%$ increased spine density and decreased spine motility on L5 cortical pyramidal neurons of PirB KO mice. These observations can explain the increased sensory-driven plasticity (Djurisic et al., 2013). However, OD-plasticity induced after MD was mediated via a rapid strengthening of open eye responses, which is a sign for the adult type of OD-plasticity and hence very different from the observed juvenile-like OD-plasticity in PSD-95 deficient mice during this project. Therefore, OD-plasticity is possibly induced via a different mechanism.

Nevertheless, further experiments will be needed to clarify the role of Nogo-66 receptor and PirB for the experience-dependent maturation of silent synapses as well as its interplay with inhibitory circuit maturation (Chevaleyre et al., 2004; Morishita et al., 2010). A functional or molecular interaction of these proteins with PSD-95 is yet unknown and both proteins are influencing structural plasticity of the extracellular matrix (ECM) as well as axon sprouting and long-term synaptic plasticity. The mature extracellular matrix acts as an inhibitor for experience-dependent plasticity. It has been shown that chondroitin sulphate proteoglycans (CSPGs), which are components of the ECM, can inhibit axonal sprouting. Thus, their degradation with the enzyme chondroitinase-ABC can reportedly reactivate OD- plasticity in adult rats (Pizzorusso et al., 2002). The organization of CSPGs into perineuronal nets matches with the end of the CP for OD-plasticity and is delayed by dark rearing, which supports the assumption that this event represents an experience-dependent process (Pizzorusso et al., 2002). 
In conclusion, this PhD thesis presents evidence of an association of the function of PSD-95 with experience-dependent maturation of silent synapses during the CP for OD-plasticity. Thereby, the described findings demonstrate an essential function of silent synapses in neural network refinement. Particular emphasis should therefore be given to the fact that the observed conversion of silent synapses into transmitting synapses might represent the terminating event for the critical period. Despite the normal increase of the local inhibitory tone in PSD-95 KO mice during development, the time window for OD-plasticity remains open. Even more noteworthy, it can be reopened when silent synapses are restored by knocking down PSD-95 in the visual cortex of adult mice. Hence, this result shows that ODplasticity can persist even if there are high levels of inhibition present.

Neuronal plasticity is, however, not only important during the developmental process but also in cases of brain damage from injury, such as a stroke. It was previously reported that ODplasticity is lost in the adult mouse primary visual cortex (V1) after a photothrombotic (PT) stroke lesion in the primary somatosensory cortex (S1) (Greifzu et al., 2011). However, this lack of OD-plasticity after a cortical lesion can be preserved by raising mice in an enriched environment (Greifzu et al., 2014) or by short-term dark exposure (Stodieck et al., 2014). The primary mechanism behind these observations is mainly a reduction of the inhibitory tone in V1. Surprisingly, PSD-95 KO mice still showed OD-plasticity after a lesion in S1, which was tested with intrinsic signal optical imaging (Greifzu et al., 2016). However, the experienceenabled enhancement of the optomotor reflex tested in the open eye after MD was compromised in both S1-lesioned PSD-95 KO and WT mice. These results indicate that after a stroke in S1, OD-plasticity can either be fostered by changes in excitatory circuitry (Greifzu et al., 2016) or by reducing the inhibitory tone in V1 (Greifzu et al., 2014; Stodieck et al., 2014). Noteworthy, elevated numbers of AMPA-silent synapses cannot only preserve OD-plasticity in the adult and healthy brain, but also in the brain suffering from a cortical stroke in S1.

The attributes of a lack of PSD-95 and hence increased plasticity and recovery from brain injury seem to be beneficial at first sight, but might also have a downside. Neural circuits are not only highly plastic in PSD-95 deficient mice but also less stable as observed in PSD-95 KO mice during the course of this project (Huang et al., 2015) (Figure 29). In 2007, it was already shown by Ehrlich et al. that after LTP induction in brain slice cultures with an acute knockdown of PSD-95 by RNAi, potentiated spines are highly labile, while LTP was shown to be enhanced in the hippocampus of PSD-95 KO mice (Migaud et al., 1998). These results point out that the 
synaptic maturation during the critical period is unstable and only temporary in PSD-95 KO mice. The observation made in 3.2. of reopening the initially deprived eye for two days after seven days of MD in adult PSD-95 KO mice (Figure 28) are in line with these previous studies. In fact, these present observations show that plastic changes in V1 of PSD-95 KO mice are more transient and return faster to the initial state than in WT mice. This indicates that functional properties of neurons and synaptic changes during experience-dependent network refinements cannot be stabilized without PSD-95. Consequently, it can be proposed that PSD95 deficient mice exhibit an extremely unstable network, which can adjust rapidly according to its experience but cannot preserve these changes, resulting in a deficit of sensory functions. The critical period for OD-plasticity reportedly matches the exact point of time at which temporal regulations drive binocular matching of orientation preference in mouse V1 (Wang et al., 2010; Wang et al., 2013). It was shown that binocular matching is permanently disrupted when the critical period starts precociously by genetically enhancing inhibition in mice. Hence, proper timing of the critical period is essential for establishing normal binocularity (Wang et al., 2010; Wang et al., 2013). In addition to binocularity, cortical cells are also selective for stimulus orientation (Ferster and Miller, 2000; Hubel and Wiesel, 1962) and binocular cells must match their orientation tuning through the pair of eyes in order to perceive accurately. Therefore, the increased plasticity during the critical period allows visual experience to drive the binocular matching of orientation preference during normal development. It is suggested that the critical period for binocular matching of orientation preference is crucially needed for the normal development of cortical binocularity. However, once it is fully established after the critical period, it cannot be disrupted anymore by MD (Wang et al., 2010). In line with this, dark rearing from birth delayed the critical period for the matching process similarly to that of OD-plasticity (Wang et al., 2010). Additionally, it was shown that binocular matching requires NMDA receptor activation (Kleinschmidt et al., 1987; Roberts et al., 1998; Sato and Stryker, 2008), which presumably detects the correlation between the inputs of the two eyes. This appears to be a rather sophisticated mechanism instead of being determined exclusively by genetic programs. Since animals exhibit different features, e.g., in their physique and differences in head size or eye position, it is necessary to adjust the neural circuits for binocular vision to their individual anatomical disparities in order to form a coherent visual perception (Wang et al., 2010). 
Based on the reported lifelong and prolonged critical period for OD-plasticity in PSD-95 KO mice (Huang et al., 2015) (chapters 3.1. - 3.9.), it can be presumed that binocular matching is also not fully taking place in these mice when the critical period continuously proceeds.

In fact, PSD-95 is not only present in the brain but also in horizontal cells, amacrine cells and ganglion cells of the retina (Koulen et al., 1998). Addressing these observations, the laboratory under the direction of Prof. Dr. Löwel (Götze and Wenzel), investigated vision in PSD-95 KO mice by testing them in the visual water task (Prusky et al., 2000). In comparison to WT mice, PSD-95 KO mice exhibited normal visual acuity (Huang et al., 2015) but showed impaired orientation discrimination. This correlates with the findings of Fagiolini et al. (2003), who were able to show with in vivo single-unit recordings that PSD-95 KO mice exhibit fewer orientationbiased cells in the visual cortex compared to WT littermates.

According to these results, it was of interest to test whether WT mice with a specific knockdown of PSD-95 restricted to the visual cortex would also show normal visual acuity or impaired orientation discrimination tested in the visual water task.

Contrary to the work hypothesis of this PhD thesis, both WT mice with a visual cortex restricted knockdown of PSD-95 after birth (P $0-P 2$ ) and in adulthood ( P 140) revealed normal visual acuity and orientation discrimination in comparison to controls which was tested with the visual water task (Prusky et al., 2000) (Figure 39). Thus, there is an obvious discrepancy in orientation discrimination of ubiquitous PSD-95 KO mice as observed during this project compared to WT mice with a site-specific knockdown of PSD-95 only in the visual cortex. There are two possible explanations to interpret this discrepancy: Either the transfection efficiency of the injected virus was insufficient to elicit a behavioral change in the visual water task or there could be other brain regions involved in which PSD-95 is responsible for normal orientation discrimination. The superior colliculus directly gets retinal projections (Attardi \& Sperry, 1963; Dräger, Hubel, 1975; Review: Hubermann \& Niell, 2011). Moreover, it exhibits orientation columns (Feinberg \& Meister, 2015; Ahmadlou \& Heimel, 2015). Thus, a potential explanation could be a possible and yet unreported importance of the superior colliculus for normal orientation discrimination. However, weighing these two possibilities against each other, an insufficient virus transfection appears to be more likely. In fact, the knockdown of PSD-95 in the visual cortex was sufficient to elicit OD-plasticity in WT mice of all tested ages (Figure 32, Figure 35, Figure 41), but the transfection efficiency remained presumably not sufficient enough to trigger impaired orientation discrimination. Testing the 
transfection efficiency in brain slices revealed no homogenous distribution of the virus in the visual cortex and variation between the different animals could be observed. Furthermore, our collaborating group under the direction of Prof. Dr. Dr. Schlüter repeated these experiments and could indeed find impaired orientation discrimination in visual cortex restricted PSD-95 knockdown mice (preliminary data).

In summary, the above described experiments provide evidence that PSD-95 controls the ending of the CP for juvenile OD-plasticity by silent synapse maturation. This is supported by the observations that a lack of PSD-95 leads to lifelong juvenile-like OD-plasticity as well as steady high levels of silent synapse numbers in the visual cortex (Huang et al., 2015). It is noteworthy that visual cortex restricted knockdowns of PSD-95 at birth (P 0) (chapter 3.3), after the CP (P 40) (chapter 3.5.) or in adulthood (> P 110) (chapter 3.8.) robustly preserved juvenile-like OD-plasticity in WT mice. This was also observed in mice with a site-specific PSD95 knockdown restricted to the contralateral visual cortex of the deprived eye. In contrast to the current view that increased intracortical inhibition is the major player in closing the critical period, the findings of this $\mathrm{PhD}$ thesis allow the opposing assumption that the closure is mainly based on the maturation of silent synapses and mostly independent of the inhibitory tone. However, it remains to be tested if and to what extent extracellular matrix structures like perineuronal nets or myelin, which are known to restrict neuronal plasticity (Berardi et al., 2004; Sugiyama et al., 2009; Pizzorusso et al., 2002; McGee et al., 2005; Atwal et al., 2008), play a role in the lifelong juvenile-like critical period for OD-plasticity in PSD-95 KO mice. It would be interesting to elucidate further if PSD-95 KO or knockdown mice show less PNN numbers in the visual cortex than WT mice and to investigate potentially accompanying structural changes of the ECM. Furthermore, additional experiments will be necessary to clarify the role of the interplay between inhibitory circuit maturation and unsilencing of excitatory synapses and the function of Nogo-66 receptor and PirB for experience-dependent maturation of silent synapses (Djurisic et al., 2013; Syken et al., 2006; Atwal et al., 2008; McGee et al., 2005; Morishita et al., 2010; Chevaleyre and Castillo, 2004). 


\subsection{PSD-93}

While PSD-95 was extensively studied in the past years, only little is known about the postsynaptic density protein-93 (PSD-93). It is postulated that PSD-93 is essential for surface NMDAR expression, NMDAR-mediated postsynaptic function and NMDAR-dependent persistent pain (Tao et al., 2003). PSD-93 KO mice are reported to have impaired NMDARmediated postsynaptic functions and exhibit a truncation in NMDAR-dependent persistent pain by mechanisms of surface NMDAR alteration (Tao et al., 2003).

However, knocking out PSD-93 in mice revealed no structural or functional abnormalities in cerebellar purkinje neurons (Mc Gee et al., 2001) and was reported to be rather redundant in comparison to PSD-95 (Carlisle et al., 2008; Krüger et al., 2013) or suggested to have similar roles in AMPAR neurotransmission in hippocampal synapses (Elias et al., 2006; Elias and Nicoll, 2007). Additionally, it was suggested that a knockdown of each of the membraneassociated guanylate kinases (MAGUK) proteins caused a similar decrease in glutamatergic transmission, which included reductions of approx. $50 \%$ in AMPAR and $25 \%$ in NMDAR transmission (Levy et al., 2015). In the same study, it was suggested that the deficit in glutamatergic current is mediated by a postsynaptic reduction in the number of functional synapses and a triple knockdown of PSD-93, PSD-95 and SAP 102, which caused an $80 \%$ reduction in both AMPAR and NMDAR synaptic responses.

Together with our collaborating group under the direction of Prof. Dr. Dr. Schlüter (ENI, Göttingen), we challenge this controversial view on the role of PSD-93 by proposing that both PSD-95 and PSD-93 are key scaffolds in the postsynaptic density of excitatory synapses with opposing functions. This would suggest that both proteins orchestrate the appropriate maturation of AMPA receptor neurotransmission in the visual cortex (Huang et al., 2015, Favaro, doctoral thesis, 2014).

In comparison to PSD-95, PSD-93 is responsible for keeping the number of AMPAR silent synapses at a steady level (Favaro, 2014). A knockout of PSD-93 in mice was reported to rapidly reduce the number of AMPAR silent synapses during development and diminish their number to nearly $0 \%$ already during the critical period. Electrophysiological data also revealed that synaptic maturation is faster in PSD-93 KO mice (Favaro, unpublished). This was indicated by a loss of silent synapses already during the critical period (Figure 53) accompanied by a functional increase in the strength of AMPA receptor neurotransmission in 
synapses of layers 4 to $2 / 3$ synapses. Furthermore, there was no change in neurotransmitter release or basal NMDA receptor neurotransmission. This suggests that PSD-93 is the opponent of PSD-95 and both proteins are important for maintaining brain plasticity until optimal function is achieved. Subsequently, brain plasticity decreases to maintain the best functional state in the given environment.

Like the above mentioned electrophysiological data, experiments described in chapter 3.15. of this PhD thesis led to the observation that OD-plasticity was completely absent in PSD-93 KO mice after $\mathrm{P}$ 27. This observation was already made during the critical period for ODplasticity (P 28 - 35), whereas OD-plasticity persists in age-matched WT mice after 4 days of $M D$. These results allow the assumption that there is an earlier closure of the CP for ODplasticity around P 27 in the absence of PSD-93. This matches with the previously described electrophysiological data of reduced AMPAR silent synapses in PSD-93 KO mice (Favaro, 2014).

Manipulating the point of time of the critical period expression, for instance prolonging it, can be achieved by raising standard cage WT mice in complete darkness (DR) from birth. It was reported that dark rearing increased OD-plasticity and prolongs the critical period for ODplasticity (Fagiolini et al., 1994; Cynader et al., 1983). Funahashi et al. (2013) could show that during mouse development silent synapses were largely present before eye opening, decreased significantly during the critical period of OD-plasticity and were almost absent in adulthood. However, this decline of silent synapse numbers during development was prevented by dark rearing. Based on these findings, Favaro (Schlüter laboratory, ENI, Göttingen) investigated the number of silent synapses in dark reared PSD-93 KO mice. Surprisingly, this work revealed that even dark rearing cannot prevent the rapid decline of silent synapse numbers in PSD-93 KO mice which were already absent during the critical period (unpublished data).

Simultaneously, optical imaging of intrinsic signals of dark reared PSD-93 KO and WT control mice was performed in this study after 4 days of MD between P $28-35$. Indeed, optical imaging revealed that PSD-93 KO mice do not show OD-plasticity even after dark rearing during the late critical period (P 28 - 35), whereas age-matched dark reared WT mice still exhibited OD-plasticity (Figure 64). Even beyond the CP (P 40 - 50), where OD-plasticity usually cannot be induced after four days of MD in standard cage raised WT mice anymore (Lehmann and Löwel, 2008), dark reared WT mice still showed a strong OD-shift. This is supported by 
work in mice (Review: Hooks \& Chen, 2007), cats (Mower et al., 1981; Mower et al., 1991; Cynader, 1983) and rats (Fagiolini et al., 1994, Morales et al., 2002). The strong OD-shift was mediated by a reduction of deprived eye responses whereas ipsilateral evoked responses in V1 remained unchanged (Figure 64). Thus, characteristic features of juvenile OD-plasticity were present in WT mice during the CP. They were also observable beyond the critical period when these WT mice were reared in darkness, whereas OD-plasticity was completely absent in PSD-93 KO mice after P 27 (Figure 59). These findings indicate that the critical period plasticity in V1 closes earlier in PSD-93 KO mice and cannot be rescued by dark rearing. This suggests that the faster maturation of PSD-93 KO mice is not an experience-dependent process.

Since it was shown in this work that a visual cortex restricted knockdown of PSD-95 is sufficient to induce juvenile-like OD-plasticity in WT mice (Huang et al., 2015), it was furthermore investigated whether site-specific PSD-93 manipulations restricted to the visual cortex were sufficient to induce an earlier closure of the critical period for OD-plasticity in WT mice. In mice with a visual cortex specific PSD-93 knockdown, 4 days of MD could not induce an OD-shift, whereas it was present in age-matched control mice which showed a strong ODshift after four days of MD. This result is consistent with the previous observation of 4 days of MD being not sufficient to induce OD-plasticity in PSD-93 deficient mice in the visual cortex during the late critical period beyond P 27.

To test whether the visual cortex of PSD-93 KO mice is similarly organized to that of WT mice, V1 topographic maps were analyzed by optical imaging of intrinsic signals which revealed no difference between both mouse strains (Figure 74). Visual abilities of PSD-93 KO and WT mice were tested in the visual water task (VWT). PSD-93 KO mice had a similar visual acuity limit to that of WT mice (Figure 52). This finding demonstrates that PSD-93 KO mice are as good as WT mice in learning an associative visual task. It also indicates that their visual acuity is not compromised.

Furthermore, the orientation discrimination of PSD-93 KO and WT mice was tested, which proved to be significantly impaired in PSD-93 KO mice compared to that of WT mice (Figure 52). One explanation for similar visual acuity but compromised orientation discrimination in PSD-93 KO mice could be that the brain is maturing too fast compared to that of WT mice. Additionally, networks responsible for orientation discrimination and binocular matching 
cannot be established properly to gain their optimal functional properties which usually occur during the critical period.

Furthermore, the effect of voluntary physical exercise on the development of PSD-93 KO mice was examined by raising the mice in running wheel cages. Several studies stated that environmental enrichment improves cortical plasticity in both adult rats (Sale et al., 2007) and in mice where juvenile-like OD-plasticity was preserved into late adulthood (Greifzu et al., 2014). Likewise, running alone is reported to increase pyramidal cell firing and sensory responses of neurons in the VC of mice (Niell and Stryker, 2010; Fu et al., 2014). Recently, it was shown that juvenile-like OD-plasticity is enhanced and restored in WT mice after voluntary physical exercise (Kalogeraki et al., 2014). This was accomplished by raising mice in a cage with a running wheel or allowing them to run for 7 days during the MD-period. Hence, physical exercise alone is already sufficient to promote plasticity in WT mice. Interestingly, during this project it was observed that already during the late critical period CP (P 28

- 34) OD-plasticity was completely abolished in PSD-93 KO mice. Moreover, this was also observed even after voluntary physical exercise (Figure 66). This observation indicates that there might be an earlier closure of the critical period for OD-plasticity at around P 27 in the absence of PSD-93. Notably, this earlier closure cannot be preserved by running or by dark rearing, which both were shown before to be beneficial in WT mice (Kalogeraki et al., 2014; Funahashi et al., 2013).

Under consideration of the above described observations, a novel scenario for the role of PSD-95 and PSD-93 in synaptic maturation and OD-plasticity in mice can be proposed, in which both act as opponents. Subsequently, the assumption can be made that PSD-93 is responsible for preventing the early synaptic maturation in the visual cortex and its lack results in an earlier maturation. Hence, a precocious closure of the critical period for OD- plasticity in mice can be initially observed during the critical period. In contrast, PSD-95 counteracts this mode of action by promoting synaptic maturation and stabilization of AMPAR at postsynaptic sites. This notion is further underlined by the discovery that a double KO of PSD-95 and PSD-93 exhibits normal numbers of AMPAR silent synapses in the visual cortex of mice, which were similar to observed silent synapse numbers in control WT mice (Favaro, 2014).

In summary, the present PhD thesis provides evidence that PSD-95 controls the ending of the critical period for juvenile OD-plasticity, whereas PSD-93 exhibits the opposing function. In 
contrast to the current view that increased intracortical inhibition is the major player in closing the critical period, the findings of this PhD study allow the opposing assumption that the closure is mainly based on PSD-95 function and mostly independent of the inhibitory tone. Taken together, these results expand the knowledge about the molecular mechanisms underlying synaptic maturation in the visual cortex and enrich the current view concerning the roles of PSD-95 and PSD-93 and its functional interactions regulating OD-plasticity in mice. 


\section{Abbreviation list}

\begin{tabular}{|c|c|}
\hline AMPA & $\alpha$-amino-3-hydroxy-5-methyl-4-isoxazolepropionic acid \\
\hline AMPAR & $\alpha$-amino-3-hydroxy-5-methyl-4-isoxazolepropionic acid receptor \\
\hline BDNF & brain-derived neurotropic factor \\
\hline $\mathrm{CP}$ & critical period \\
\hline cyc/deg & cycles per degree \\
\hline EE & enriched environment \\
\hline GABA & $\gamma$-aminobutyric acid \\
\hline GK & guanylate kinase \\
\hline IGF & insulin-like growth factor \\
\hline LGN & lateral geniculate nucleus \\
\hline L4-L2/3 & layer 4 to layers $2 / 3$ \\
\hline LTD & long-term depression \\
\hline LTP & long-term potentiation \\
\hline MD & monocular deprivation \\
\hline $\mathrm{mg}$ & milligram \\
\hline $\mathrm{ml}$ & milliliter \\
\hline $\mathrm{mm}$ & millimeter \\
\hline mEPSC & miniature excitatory postsynaptic current \\
\hline $\mathrm{n}$ & number of animals \\
\hline $\mathrm{N}_{2} \mathrm{O}$ & nitrous oxide \\
\hline NMDA & $\mathrm{N}$-methyl-D-aspartate \\
\hline NMDAR & $\mathrm{N}$-methyl-D-aspartate receptor \\
\hline $\mathrm{O}_{2}$ & oxygen \\
\hline OD & ocular dominance \\
\hline ODI & ocular dominance index \\
\hline$P$ & postnatal days \\
\hline PNN & perineuronal nets \\
\hline PT & photothrombosis \\
\hline PV & parvalbumin \\
\hline PKA & protein kinase $A$ \\
\hline
\end{tabular}


PSD

PSD-93

PSD-95

RW

SAP 97

SAP 102

SEM

$\mathrm{SH} 3$

shRNA

S1

SC

TARP

V1

VC

VWT postsynaptic density

postsynaptic density protein 93

postsynaptic density protein 95

running wheel

synapse-associated protein 97

synapse-associated protein 102

standard error of the mean

Src-homology domain 3

short-hairpin RNA

primary somatosensory cortex

standard cage

transmembrane AMPAR regulatory protein

primary visual cortex

visual cortex

visual water task 


\section{References}

Ahmadlou M, Heimel JA (2015) Preference for concentric orientations in the mouse superior colliculus. Nature communications 6:6773

Akgul G, Wollmuth LP (2010) Expression pattern of membrane-associated guanylate kinases in interneurons of the visual cortex. Journal of Comparative Neurology 518:48424854.

Albus K, Wolf W (1984) Early post-natal development of neuronal function in the kitten's visual cortex: a laminar analysis. The Journal of physiology 348:153.

Antonini A, Fagiolini M, Stryker MP (1999) Anatomical correlates of functional plasticity in mouse visual cortex. The Journal of neuroscience 19:4388-4406.

Ashby MC, Isaac JT (2011) Maturation of a recurrent excitatory neocortical circuit by experience-dependent unsilencing of newly formed dendritic spines. Neuron 70:510521.

Attardi DG, Sperry R (1963) Preferential selection of central pathways by regenerating optic fibers. Experimental neurology 7:46-64.

Atwal JK, Pinkston-Gosse J, Syken J, Stawicki S, Wu Y, Shatz C, Tessier-Lavigne M (2008) PirB is a functional receptor for myelin inhibitors of axonal regeneration. Science 322:967970.

Baroncelli L, Braschi C, Spolidoro M, Begenisic T, Maffei L, Sale A (2011) Brain plasticity and disease: a matter of inhibition. Neural plasticity 2011:286073.

Bartoletti A, Cancedda L, Reid SW, Tessarollo L, Porciatti V, Pizzorusso T, Maffei L (2002) Heterozygous knock-out mice for brain-derived neurotrophic factor show a pathwayspecific impairment of long-term potentiation but normal critical period for monocular deprivation. The Journal of neuroscience 22:10072-10077.

Bats C, Groc L, Choquet D (2007) The interaction between Stargazin and PSD-95 regulates AMPA receptor surface trafficking. Neuron 53:719-734.

Bavelier D, Levi DM, Li RW, Dan Y, Hensch TK (2010) Removing brakes on adult brain plasticity: from molecular to behavioral interventions. The Journal of neuroscience 30:1496414971.

Bear M, Kleinschmidt A, Gu Q, Singer W (1990) Disruption of experience-dependent synaptic modifications in striate cortex by infusion of an NMDA receptor antagonist. The Journal of neuroscience 10:909-925.

Bear MF, Rittenhouse CD (1999) Molecular basis for induction of ocular dominance plasticity. Journal of neurobiology 41:83-91.

Béïque J-C, Lin D-T, Kang M-G, Aizawa H, Takamiya K, Huganir RL (2006) Synapse-specific regulation of AMPA receptor function by PSD-95. Proceedings of the National Academy of Sciences 103:19535-19540.

Béique JC, Andrade R (2003) PSD-95 regulates synaptic transmission and plasticity in rat cerebral cortex. The Journal of physiology 546:859-867.

Berardi N, Pizzorusso T, Maffei L (2000) Critical periods during sensory development. Current Opinion in Neurobiology 10:138-145. 
Berardi N, Pizzorusso T, Maffei L (2004) Extracellular matrix and visual cortical plasticity: freeing the synapse. Neuron 44:905-908.

Bhattacharyya S, Biou V, Xu W, Schlüter O, Malenka RC (2009) A critical role for PSD-95/AKAP interactions in endocytosis of synaptic AMPA receptors. Nature neuroscience 12:172181.

Blais BS, Frenkel MY, Kuindersma SR, Muhammad R, Shouval HZ, Cooper LN, Bear MF (2008) Recovery from monocular deprivation using binocular deprivation. Journal of Neurophysiology 100:2217-2224.

Blakemore C (1976) The conditions required for the maintenance of binocularity in the kitten's visual cortex. The Journal of physiology 261:423.

Blakemore C, Van Sluyters RC (1975) Innate and environmental factors in the development of the kitten's visual cortex. J Physiol 248:663-716.

Blanpied TA, Kerr JM, Ehlers MD (2008) Structural plasticity with preserved topology in the postsynaptic protein network. Proceedings of the National Academy of Sciences 105:12587-12592.

Blasdel GG, Salama G (1986) Voltage-sensitive dyes reveal a modular organization in monkey striate cortex. Nature 321:579-585.

Blue ME, Parnavelas JG (1983) The formation and maturation of synapses in the visual cortex of the rat. I. Qualitative analysis. Journal of neurocytology 12:599-616.

Bonhoeffer T, Grinvald A (1993) The layout of iso-orientation domains in area 18 of cat visual cortex: optical imaging reveals a pinwheel-like organization. The Journal of neuroscience 13:4157-4180.

Bonnet SA, Akad DS, Samaddar T, Liu Y, Huang X, Dong Y, Schlüter OM (2013) Synaptic statedependent functional interplay between postsynaptic density-95 and synapseassociated protein 102. The Journal of neuroscience 33:13398-13409.

Boothe RG, Dobson V, Teller DY (1985) Postnatal development of vision in human and nonhuman primates. Annu Rev Neurosci 8:495-545.

Bourgeois J (1997) Synaptogenesis, heterochrony and epigenesis in the mammalian neocortex. Acta Paediatrica 86:27-33.

Bredt DS, Nicoll RA (2003) AMPA receptor trafficking at excitatory synapses. Neuron 40:361379.

Cang J, Kalatsky VA, Löwel S, Stryker MP (2005a) Optical imaging of the intrinsic signal as a measure of cortical plasticity in the mouse. Visual neuroscience 22:685-691.

Cang J, Kaneko M, Yamada J, Woods G, Stryker MP, Feldheim DA (2005b) Ephrin-as guide the formation of functional maps in the visual cortex. Neuron 48:577-589.

Cang J, Rentería RC, Kaneko M, Liu X, Copenhagen DR, Stryker MP (2005c) Development of precise maps in visual cortex requires patterned spontaneous activity in the retina. Neuron 48:797-809.

Carlisle HJ, Fink AE, Grant SG, O'Dell TJ (2008) Opposing effects of PSD-93 and PSD-95 on longterm potentiation and spike timing-dependent plasticity. The Journal of physiology 586:5885-5900. 
Carmignoto G, Vicini S (1992) Activity-dependent decrease in NMDA receptor responses during development of the visual cortex. Science 258:1007-1011.

Charych El, Akum BF, Goldberg JS, Jörnsten RJ, Rongo C, Zheng JQ, Firestein BL (2006) Activityindependent regulation of dendrite patterning by postsynaptic density protein PSD95. The Journal of neuroscience 26:10164-10176.

Chen L, Chetkovich DM, Petralia RS, Sweeney NT, Kawasaki Y, Wenthold RJ, Bredt DS, Nicoll RA (2000) Stargazin regulates synaptic targeting of AMPA receptors by two distinct mechanisms. Nature 408:936-943.

Chen X, Levy JM, Hou A, Winters C, Azzam R, Sousa AA, Leapman RD, Nicoll RA, Reese TS (2015) PSD-95 family MAGUKs are essential for anchoring AMPA and NMDA receptor complexes at the postsynaptic density. Proceedings of the National Academy of Sciences 112: E6983-E6992.

Chen X, Nelson CD, Li X, Winters CA, Azzam R, Sousa AA, Leapman RD, Gainer H, Sheng M, Reese TS (2011) PSD-95 is required to sustain the molecular organization of the postsynaptic density. The Journal of neuroscience 31:6329-6338.

Chetkovich DM, Chen L, Stocker TJ, Nicoll RA, Bredt DS (2002) Phosphorylation of the postsynaptic density-95 (PSD-95)/discs large/zona occludens-1 binding site of stargazin regulates binding to PSD-95 and synaptic targeting of AMPA receptors. The Journal of neuroscience 22:5791-5796.

Chevaleyre V, Castillo PE (2004) Endocannabinoid-mediated metaplasticity in the hippocampus. Neuron 43:871-881.

Cho K-O, Hunt CA, Kennedy MB (1992) The rat brain postsynaptic density fraction contains a homolog of the Drosophila discs-large tumor suppressor protein. Neuron 9:929-942.

Ciucci F, Putignano E, Baroncelli L, Landi S, Berardi N, Maffei L (2007) Insulin-like growth factor 1 (IGF-1) mediates the effects of enriched environment (EE) on visual cortical development. PloS one 2: e475.

Colledge M, Snyder EM, Crozier RA, Soderling JA, Jin Y, Langeberg LK, Lu H, Bear MF, Scott JD (2003) Ubiquitination regulates PSD-95 degradation and AMPA receptor surface expression. Neuron 40:595-607.

Collingridge GL, Singer W (1990) Excitatory amino acid receptors and synaptic plasticity. Trends in pharmacological sciences 11:290-296.

Crair MC, Malenka RC (1995) A critical period for long-term potentiation at thalamocortical synapses. Nature 375:325-328.

Craven SE, Bredt DS (1998) PDZ proteins organize synaptic signaling pathways. Cell 93:495498.

Craven SE, El-Husseini AE, Bredt DS (1999) Synaptic targeting of the postsynaptic density protein PSD-95 mediated by lipid and protein motifs. Neuron 22:497-509.

Cudeiro J, Sillito AM (2006) Looking back: corticothalamic feedback and early visual processing. Trends in neurosciences 29:298-306.

Cuthbert PC, Stanford LE, Coba MP, Ainge JA, Fink AE, Opazo P, Delgado JY, Komiyama NH, O'Dell TJ, Grant SG (2007) Synapse-associated protein 102/dlgh3 couples the NMDA 
receptor to specific plasticity pathways and learning strategies. The Journal of neuroscience 27:2673-2682.

Cynader M (1983) Prolonged sensitivity to monocular deprivation in dark-reared cats: effects of age and visual exposure. Developmental brain research 8:155-164.

Dakoji S, Tomita S, Karimzadegan S, Nicoll RA, Bredt DS (2003) Interaction of transmembrane AMPA receptor regulatory proteins with multiple membrane associated guanylate kinases. Neuropharmacology 45:849-856.

Daw NW (1998) Critical periods and amblyopia. Archives of ophthalmology 116:502-505. Daw NW, Fox K, Sato H, Czepita D (1992) Critical period for monocular deprivation in the cat visual cortex. Journal of Neurophysiology 67:197-202.

Defelipe J, Markram H, Rockland KS (2012) The neocortical column. Frontiers in Neuroanatomy 6(22):1-2.

DeGiorgis JA, Galbraith JA, Dosemeci A, Chen X, Reese TS (2006) Distribution of the scaffolding proteins PSD-95, PSD-93, and SAP97 in isolated PSDs. Brain cell biology 35:239-250.

Dehmel S, Löwel S (2014) Cortico-Cortical Interactions Influence Binocularity of the Primary Visual Cortex of Adult Mice. PloS one 9:e105745.

Di Cristo G, Chattopadhyaya B, Kuhlman SJ, Fu Y, Bélanger M-C, Wu CZ, Rutishauser U, Maffei L, Huang ZJ (2007) Activity-dependent PSA expression regulates inhibitory maturation and onset of critical period plasticity. Nature neuroscience 10:1569-1577.

Djurisic M, Vidal GS, Mann M, Aharon A, Kim T, Santos AF, Zuo Y, Hübener M, Shatz CJ (2013) PirB regulates a structural substrate for cortical plasticity. Proceedings of the National Academy of Sciences 110:20771-20776.

Dong Y, Graziane N (2016) Measurement of Silent Synapses. Electrophysiological Analysis of Synaptic Transmission:217-224.

Douglas R, Alam N, Silver B, McGill T, Tschetter W, Prusky G (2005) Independent visual threshold measurements in the two eyes of freely moving rats and mice using a virtualreality optokinetic system. Visual neuroscience 22:677-684.

Dowling J, Boycott B (1966) Organization of the primate retina: electron microscopy. Proceedings of the Royal Society of London B: Biological Sciences 166:80-111.

Dräger U, Hubel DH (1975a) Responses to visual stimulation and relationship between visual, auditory, and somatosensory inputs in mouse superior colliculus. Journal of Neurophysiology 38:690-713.

Dräger UC (1975) Receptive fields of single cells and topography in mouse visual cortex. Journal of Comparative Neurology 160:269-289.

Dräger UC, Hubel DH (1975b) Physiology of visual cells in mouse superior colliculus and correlation with somatosensory and auditory input. Nature 253:203-204.

Dräger UC, Hubel DH (1978) Studies of visual function and its decay in mice with hereditary retinal degeneration. Journal of Comparative Neurology 180:85-114.

Dräger UC, Olsen JF (1980) Origins of crossed and uncrossed retinal projections in pigmented and albino mice. Journal of Comparative Neurology 191:383-412. 
Durand G, Kenneth A (1996) Long-term potentiation as a mechanism of functional synapse induction in the developing hippocampus. Journal of Physiology-Paris 90:313-315.

Ehrensperger M-V, Hanus C, Vannier C, Triller A, Dahan M (2007) Multiple association states between glycine receptors and gephyrin identified by SPT analysis. Biophysical journal 92:3706-3718.

Ehrlich I, Malinow R (2004) Postsynaptic density 95 controls AMPA receptor incorporation during long-term potentiation and experience-driven synaptic plasticity. The Journal of neuroscience 24:916-927.

Ehrlich I, Klein M, Rumpel S, Malinow R (2007) PSD-95 is required for activity-driven synapse stabilization. Proceedings of the National Academy of Sciences 104:4176-4181.

El-Husseini AE-D, Schnell E, Chetkovich DM, Nicoll RA, Bredt DS (2000) PSD-95 involvement in maturation of excitatory synapses. Science 290:1364-1368.

El-Husseini AE-D, Schnell E, Dakoji S, Sweeney N, Zhou Q, Prange O, Gauthier-Campbell C, Aguilera-Moreno A, Nicoll RA, Bredt DS (2002) Synaptic strength regulated by palmitate cycling on PSD-95. Cell 108:849-863.

Elias GM, Nicoll RA (2007) Synaptic trafficking of glutamate receptors by MAGUK scaffolding proteins. Trends in cell biology 17:343-352.

Elias GM, Funke L, Stein V, Grant SG, Bredt DS, Nicoll RA (2006) Synapse-specific and developmentally regulated targeting of AMPA receptors by a family of MAGUK scaffolding proteins. Neuron 52:307-320.

Elstrott J, Anishchenko A, Greschner M, Sher A, Litke AM, Chichilnisky E, Feller MB (2008) Direction selectivity in the retina is established independent of visual experience and cholinergic retinal waves. Neuron 58:499-506.

Espinosa JS, Stryker MP (2012) Development and plasticity of the primary visual cortex. Neuron 75:230-249.

Esteban JA, Shi S-H, Wilson C, Nuriya M, Huganir RL, Malinow R (2003) PKA phosphorylation of AMPA receptor subunits controls synaptic trafficking underlying plasticity. Nature neuroscience 6:136-143.

Fagiolini M, Hensch TK (2000) Inhibitory threshold for critical-period activation in primary visual cortex. Nature 404:183-186.

Fagiolini M, Pizzorusso T, Berardi N, Domenici L, Maffei L (1994) Functional postnatal development of the rat primary visual cortex and the role of visual experience: dark rearing and monocular deprivation. Vision research 34:709-720.

Fagiolini M, Fritschy J-M, Löw K, Möhler H, Rudolph U, Hensch TK (2004) Specific GABAA circuits for visual cortical plasticity. Science 303:1681-1683.

Fagiolini M, Katagiri H, Miyamoto H, Mori H, Grant SG, Mishina M, Hensch TK (2003) Separable features of visual cortical plasticity revealed by $\mathrm{N}$-methyl-D-aspartate receptor $2 \mathrm{~A}$ signaling. Proceedings of the National Academy of Sciences 100:2854-2859.

Favaro PD (2014) Roles of PSD-93 and environmental enrichment in cortical synapses. PhD thesis. 
Feinberg EH, Meister M (2015) Orientation columns in the mouse superior colliculus. Nature 519:229-232.

Feng W, Zhang M (2009) Organization and dynamics of PDZ-domain-related supramodules in the postsynaptic density. Nature Reviews Neuroscience 10:87-99.

Ferster D, Miller KD (2000) Neural mechanisms of orientation selectivity in the visual cortex. Annual review of neuroscience 23:441-471.

Fitzjohn SM, Doherty AJ, Collingridge GL (2006) Promiscuous interactions between AMPA-Rs and MAGUKs. Neuron 52:222-224.

Foster M (1897) A Text Book of Physiology: The central nervous system: Macmillan.

Fregnac Y, Imbert M (1978) Early development of visual cortical cells in normal and darkreared kittens: relationship between orientation selectivity and ocular dominance. J Physiol 278:27-44.

Frenkel MY, Bear MF (2004) How monocular deprivation shifts ocular dominance in visual cortex of young mice. Neuron 44:917-923.

Fukata Y, Tzingounis AV, Trinidad JC, Fukata M, Burlingame AL, Nicoll RA, Bredt DS (2005) Molecular constituents of neuronal AMPA receptors. The Journal of cell biology 169:399-404.

Funahashi R, Maruyama T, Yoshimura Y, Komatsu Y (2013) Silent synapses persist into adulthood in layer 2/3 pyramidal neurons of visual cortex in dark-reared mice. Journal of Neurophysiology 109:2064-2076.

Gandhi SP, Yanagawa Y, Stryker MP (2008) Delayed plasticity of inhibitory neurons in developing visual cortex. Proceedings of the National Academy of Sciences 105:1679716802.

Gascon E, Vutskits L, Kiss JZ (2007) Polysialic acid-neural cell adhesion molecule in brain plasticity: from synapses to integration of new neurons. Brain research reviews 56:101-118.

Gianfranceschi L, Siciliano R, Walls J, Morales B, Kirkwood A, Huang ZJ, Tonegawa S, Maffei L (2003) Visual cortex is rescued from the effects of dark rearing by overexpression of BDNF. Proceedings of the National Academy of Sciences 100:12486-12491.

Giesemann T, Schwarz G, Nawrotzki R, Berhörster K, Rothkegel M, Schlüter K, Schrader N, Schindelin H, Mendel RR, Kirsch J (2003) Complex formation between the postsynaptic scaffolding protein gephyrin, profilin, and Mena: a possible link to the microfilament system. The Journal of neuroscience 23:8330-8339.

Gordon JA, Stryker MP (1996) Experience-dependent plasticity of binocular responses in the primary visual cortex of the mouse. The Journal of neuroscience 16:3274-3286.

Greifzu F, Parthier D, Goetze B, Schlüter OM, Löwel S (2016) Ocular Dominance Plasticity after Stroke Was Preserved in PSD-95 Knockout Mice. PloS one 11:e0149771.

Greifzu F, Schmidt S, Schmidt K-F, Kreikemeier K, Witte OW, Löwel S (2011) Global impairment and therapeutic restoration of visual plasticity mechanisms after a localized cortical stroke. Proceedings of the National Academy of Sciences 108:15450-15455. 
Greifzu F, Pielecka-Fortuna J, Kalogeraki E, Krempler K, Favaro PD, Schlüter OM, Löwel S (2014) Environmental enrichment extends ocular dominance plasticity into adulthood and protects from stroke-induced impairments of plasticity. Proceedings of the National Academy of Sciences 111:1150-1155.

Grinvald A, Lieke E, Frostig RD, Gilbert CD, Wiesel TN (1986) Functional architecture of cortex revealed by optical imaging of intrinsic signals. Nature 324:361-364.

Guo Y, Huang S, de Pasquale R, McGehrin K, Lee H-K, Zhao K, Kirkwood A (2012) Dark exposure extends the integration window for spike-timing-dependent plasticity. The Journal of neuroscience 32:15027-15035.

Han K, Kim M-H, Seeburg D, Seo J, Verpelli C, Han S, Chung HS, Ko J, Lee HW, Kim K (2009) Regulated RalBP1 binding to RalA and PSD-95 controls AMPA receptor endocytosis and LTD. PLoS Biol 7:e1000187.

Hanover JL, Huang ZJ, Tonegawa S, Stryker MP (1999) Brain-derived neurotrophic factor overexpression induces precocious critical period in mouse visual cortex. The Journal of neuroscience: the official journal of the Society for Neuroscience 19:RC40.

Harauzov A, Spolidoro M, DiCristo G, De Pasquale R, Cancedda L, Pizzorusso T, Viegi A, Berardi $N$, Maffei L (2010) Reducing intracortical inhibition in the adult visual cortex promotes ocular dominance plasticity. The Journal of neuroscience 30:361-371.

Harris KD, Mrsic-Flogel TD (2013) Cortical connectivity and sensory coding. Nature 503:51-58. $\mathrm{He} \mathrm{H-Y,} \mathrm{Hodos} \mathrm{W,} \mathrm{Quinlan} \mathrm{EM} \mathrm{(2006)} \mathrm{Visual} \mathrm{deprivation} \mathrm{reactivates} \mathrm{rapid} \mathrm{ocular} \mathrm{dominance}$ plasticity in adult visual cortex. The Journal of neuroscience 26:2951-2955.

He H-Y, Ray B, Dennis K, Quinlan EM (2007) Experience-dependent recovery of vision following chronic deprivation amblyopia. Nature neuroscience 10:1134-1136.

Hebb DO (1949) The organization of behavior: A neuropsychological approach: John Wiley \& Sons.

Hensch TK (2004) Critical period regulation. Annu Rev Neurosci 27:549-579.

Hensch TK (2005) Critical period plasticity in local cortical circuits. Nature Reviews Neuroscience 6:877-888.

Hensch TK, Fagiolini M, Mataga N, Stryker MP, Baekkeskov S, Kash SF (1998) Local GABA circuit control of experience-dependent plasticity in developing visual cortex. Science 282:1504-1508.

Heynen AJ, Yoon B-J, Liu C-H, Chung HJ, Huganir RL, Bear MF (2003) Molecular mechanism for loss of visual cortical responsiveness following brief monocular deprivation. Nature neuroscience 6:854-862.

Hofer SB, Mrsic-Flogel TD, Bonhoeffer T, Hübener M (2006) Lifelong learning: ocular dominance plasticity in mouse visual cortex. Current Opinion in Neurobiology 16:451459.

Holtmaat AJ, Trachtenberg JT, Wilbrecht L, Shepherd GM, Zhang X, Knott GW, Svoboda K (2005) Transient and persistent dendritic spines in the neocortex in vivo. Neuron 45:279-291. 
Horton JC, Hocking DR (1997) Timing of the critical period for plasticity of ocular dominance columns in macaque striate cortex. The Journal of neuroscience 17:3684-3709.

Hosang L (2014) Vision and ocular dominance plasticity of PSD-93 knockout and PSD-93/95 double knockout mice. Master thesis.

Howard MA, Elias GM, Elias LA, Swat W, Nicoll RA (2010) The role of SAP97 in synaptic glutamate receptor dynamics. Proceedings of the National Academy of Sciences 107:3805-3810.

Huang X, Stodieck SK, Goetze B, Cui L, Wong MH, Wenzel C, Hosang L, Dong Y, Löwel S, Schlüter OM (2015) Progressive maturation of silent synapses governs the duration of a critical period. Proceedings of the National Academy of Sciences 112:E3131-E3140.

Huang ZJ, Kirkwood A, Pizzorusso T, Porciatti V, Morales B, Bear MF, Maffei L, Tonegawa S (1999) BDNF regulates the maturation of inhibition and the critical period of plasticity in mouse visual cortex. Cell 98:739-755.

Hubel D, Wiesel T (1963) Shape and arrangement of columns in cat's striate cortex. The Journal of physiology 165:559.

Hubel D, Wiesel T, LeVay S (1976) Functional architecture of area 17 in normal and monocularly deprived macaque monkeys. In: Cold Spring Harbor Symposia on Quantitative Biology 40:581-589.

Hubel DH, Wiesel TN (1962) Receptive fields, binocular interaction and functional architecture in the cat's visual cortex. The Journal of physiology 160:106-154.

Hubel DH, Wiesel TN (1970) The period of susceptibility to the physiological effects of unilateral eye closure in kittens. The Journal of physiology 206:419.

Huberman AD, Niell CM (2011) What can mice tell us about how vision works? Trends in neurosciences 34:464-473.

Huopaniemi L, Keist R, Randolph A, Certa U, Rudolph U (2004) Diazepam-induced adaptive plasticity revealed by $\alpha 1$ GABAA receptor-specific expression profiling. Journal of neurochemistry 88:1059-1067.

Irie M, Hata Y, Takeuchi M, Ichtchenko K, Toyoda A, Hirao K, Takai Y, RosahI T, Südhof T (1997) Binding of neuroligins to PSD-95. Science 277:1511-1515.

Isaac JT, Nicoll RA, Malenka RC (1995) Evidence for silent synapses: implications for the expression of LTP. Neuron 15:427-434.

Isaac JT, Crair MC, Nicoll RA, Malenka RC (1997) Silent synapses during development of thalamocortical inputs. Neuron 18:269-280.

Issa NP, Trepel C, Stryker MP (2000) Spatial frequency maps in cat visual cortex. The Journal of neuroscience 20:8504-8514.

Issa NP, Trachtenberg JT, Chapman B, Zahs KR, Stryker MP (1999) The critical period for ocular dominance plasticity in the ferret's visual cortex. The Journal of neuroscience 19:69656978.

Jacobs GH (2002) Progress toward understanding the evolution of primate color vision. Evolutionary Anthropology: Issues, News, and Reviews 11:132-135. 
Jensen V, Kaiser KM, Borchardt T, Adelmann G, Rozov A, Burnashev N, Brix C, Frotscher M, Andersen P, Hvalby $\varnothing$ (2003) A juvenile form of postsynaptic hippocampal long-term potentiation in mice deficient for the AMPA receptor subunit GluR-A. The Journal of physiology 553:843-856.

Jiang B, Huang S, de Pasquale R, Millman D, Song L, Lee H-K, Tsumoto T, Kirkwood A (2010) The maturation of GABAergic transmission in visual cortex requires endocannabinoidmediated LTD of inhibitory inputs during a critical period. Neuron 66:248-259.

Kalatsky VA, Stryker MP (2003) New paradigm for optical imaging: temporally encoded maps of intrinsic signal. Neuron 38:529-545.

Kalogeraki E, Greifzu F, Haack F, Löwel S (2014) Voluntary physical exercise promotes ocular dominance plasticity in adult mouse primary visual cortex. The Journal of neuroscience 34:15476-15481.

Kameyama K, Sohya K, Ebina T, Fukuda A, Yanagawa Y, Tsumoto T (2010) Difference in binocularity and ocular dominance plasticity between GABAergic and excitatory cortical neurons. The Journal of neuroscience 30:1551-1559.

Kandel ER, O'dell TJ (1992) Are adult learning mechanisms also used for development? SCIENCE-NEW YORK THEN WASHINGTON-:243-243.

Kaneko M, Stellwagen D, Malenka RC, Stryker MP (2008a) Tumor necrosis factor- $\alpha$ mediates one component of competitive, experience-dependent plasticity in developing visual cortex. Neuron 58:673-680.

Kaneko M, Hanover JL, England PM, Stryker MP (2008b) TrkB kinase is required for recovery, but not loss, of cortical responses following monocular deprivation. Nature neuroscience 11:497-504.

Kanold PO, Kim YA, GrandPre T, Shatz CJ (2009) Co-regulation of ocular dominance plasticity and NMDA receptor subunit expression in glutamic acid decarboxylase-65 knock-out mice. The Journal of physiology 587:2857-2867.

Kaschube M (2014) Neural maps versus salt-and-pepper organization in visual cortex. Current Opinion in Neurobiology 24:95-102.

Keith DJ, El-Husseini A (2008) Excitation control: balancing PSD-95 function at the synapse. Frontiers in molecular neuroscience 1:4.

Kerr JM, Blanpied TA (2012) Subsynaptic AMPA receptor distribution is acutely regulated by actin-driven reorganization of the postsynaptic density. The Journal of neuroscience 32:658-673.

Kidd M (1962) Electron microscopy of the inner plexiform layer of the retina in the cat and the pigeon. Journal of anatomy 96:179.

Kim E, Sheng M (2004) PDZ domain proteins of synapses. Nature Reviews Neuroscience 5:771781.

Kim E, Naisbitt S, Hsueh Y-P, Rao A, Rothschild A, Craig AM, Sheng M (1997) GKAP, a novel synaptic protein that interacts with the guanylate kinase-like domain of the PSD95/SAP90 family of channel clustering molecules. The Journal of cell biology 136:669678. 
Kirkwood A, Bear MF (1994) Homosynaptic long-term depression in the visual cortex. The Journal of neuroscience 14:3404-3412.

Klausberger T, Roberts JDB, Somogyi P (2002) Cell type-and input-specific differences in the number and subtypes of synaptic GABAA receptors in the hippocampus. The Journal of neuroscience 22:2513-2521.

Kleinschmidt A, Bear MF, Singer W (1987) Blockade of "NMDA" receptors disrupts experiencedependent plasticity of kitten striate cortex. Science 238:355-358.

Ko H, Hofer SB, Pichler B, Buchanan KA, Sjostrom PJ, Mrsic-Flogel TD (2011) Functional specificity of local synaptic connections in neocortical networks. Nature 473:87-91.

Kobayashi Y, Ye Z, Hensch Takao K (2015) Clock Genes Control Cortical Critical Period Timing. Neuron 86:264-275.

Kornau H-C, Schenker LT, Kennedy MB, Seeburg PH (1995) Domain interaction between NMDA receptor subunits and the postsynaptic density protein PSD-95. Science 269:1737.

Koulen P, Fletcher EL, Craven SE, Bredt DS, Wässle H (1998) Immunocytochemical localization of the postsynaptic density protein PSD-95 in the mammalian retina. The Journal of neuroscience 18:10136-10149.

Krüger JM (2010) Expression and function of PSD-93 isoforms in hippocampal synapses. Dissertation.

Krüger JM, Favaro PD, Liu M, Kitlińska A, Huang X, Raabe M, Akad DS, Liu Y, Urlaub H, Dong Y (2013) Differential roles of postsynaptic density-93 isoforms in regulating synaptic transmission. The Journal of neuroscience 33:15504-15517.

Kuhlman SJ, Olivas ND, Tring E, Ikrar T, Xu X, Trachtenberg JT (2013) A disinhibitory microcircuit initiates critical-period plasticity in the visual cortex. Nature 501:543-546.

Kuriu T, Inoue A, Bito H, Sobue K, Okabe S (2006) Differential control of postsynaptic density scaffolds via actin-dependent and-independent mechanisms. The Journal of neuroscience 26:7693-7706.

Law CC, Cooper LN (1994) Formation of receptive fields in realistic visual environments according to the Bienenstock, Cooper, and Munro (BCM) theory. Proceedings of the National Academy of Sciences 91:7797-7801.

Law CC, Bear MF, Cooper LN (1994) Role of the visual environment in the formation of receptive fields according to the BCM theory. Progress in brain research 102:287-301.

Lee W-CA, Bonin V, Reed M, Graham BJ, Hood G, Glattfelder K, Reid RC (2016) Anatomy and function of an excitatory network in the visual cortex. Nature 532:370-374.

Lehmann K, Löwel S (2008) Age-dependent ocular dominance plasticity in adult mice. PloS one 3:e3120.

Leonard AS, Davare MA, Horne MC, Garner CC, Hell JW (1998) SAP97 is associated with the $\alpha$-amino-3-hydroxy-5-methylisoxazole-4-propionic acid receptor GluR1 subunit. Journal of Biological Chemistry 273:19518-19524.

Levelt CN, Hübener M (2012) Critical-period plasticity in the visual cortex. Annual review of neuroscience 35:309-330. 
Levy JM, Chen X, Reese TS, Nicoll RA (2015) Synaptic consolidation normalizes AMPAR quantal size following MAGUK loss. Neuron 87:534-548.

Li YT, Ibrahim LA, Liu BH, Zhang LI, Tao HW (2013) Linear transformation of thalamocortical input by intracortical excitation. Nat Neurosci 16:1324-1330.

Liao D, Hessler NA, Malinow R (1995) Activation of postsynaptically silent synapses during pairing-induced LTP in CA1 region of hippocampal slice. Nature 375:400-404.

Liaw W-J, Zhu X-G, Yaster M, Johns RA, Gauda EB, Tao Y-X (2008) Distinct expression of synaptic NR2A and NR2B in the central nervous system and impaired morphine tolerance and physical dependence in mice deficient in postsynaptic density-93 protein. Molecular pain 4:1.

Lien AD, Scanziani M (2013) Tuned thalamic excitation is amplified by visual cortical circuits. Nature neuroscience 16:1315-1323.

Liu C-H, Heynen AJ, Shuler MGH, Bear MF (2008) Cannabinoid receptor blockade reveals parallel plasticity mechanisms in different layers of mouse visual cortex. Neuron 58:340-345.

Löwel S, Singer W (1992) Selection of intrinsic horizontal connections in the visual cortex by correlated neuronal activity. Science 255:209.

Maffei L, Berardi N, Domenici L, Parisi V, Pizzorusso T (1992) Nerve growth factor (NGF) prevents the shift in ocular dominance distribution of visual cortical neurons in monocularly deprived rats. The Journal of neuroscience 12:4651-4662.

Mainardi M, Landi S, Berardi N, Maffei L, Pizzorusso T (2009) Reduced responsiveness to longterm monocular deprivation of parvalbumin neurons assessed by c-Fos staining in rat visual cortex. PloS one 4:e4342.

Malenka RC, Nicoll RA (1997) Silent synapses speak up. Neuron 19:473-476.

Malenka RC, Nicoll RA (1999) Long-term potentiation--a decade of progress? Science 285:1870-1874.

Malenka RC, Bear MF (2004) LTP and LTD: an embarrassment of riches. Neuron 44:5-21. Malinow R, Malenka RC (2002) AMPA receptor trafficking and synaptic plasticity. Annual review of neuroscience 25:103-126.

Mangini NJ, Pearlman AL (1980) Laminar distribution of receptive field properties in the primary visual cortex of the mouse. The Journal of comparative neurology 193:203222.

Mataga N, Nagai N, Hensch TK (2002) Permissive proteolytic activity for visual cortical plasticity. Proceedings of the National Academy of Sciences 99:7717-7721.

Maya-Vetencourt JF, Baroncelli L, Viegi A, Tiraboschi E, Castren E, Cattaneo A, Maffei L (2012) IGF-1 restores visual cortex plasticity in adult life by reducing local GABA levels. Neural plasticity 2012:250421.

McGee AW, Yang Y, Fischer QS, Daw NW, Strittmatter SM (2005) Experience-driven plasticity of visual cortex limited by myelin and Nogo receptor. Science 309:2222-2226. 
McGee AW, Dakoji SR, Olsen O, Bredt DS, Lim WA, Prehoda KE (2001a) Structure of the SH3guanylate kinase module from PSD-95 suggests a mechanism for regulated assembly of MAGUK scaffolding proteins. Molecular cell 8:1291-1301.

McGee AW, Topinka JR, Hashimoto K, Petralia RS, Kakizawa S, Kauer F, Aguilera-Moreno A, Wenthold RJ, Kano M, Bredt DS (2001b) PSD-93 knock-out mice reveal that neuronal MAGUKs are not required for development or function of parallel fiber synapses in cerebellum. The Journal of neuroscience 21:3085-3091.

Metin C, Godement P, Imbert M (1988) The primary visual cortex in the mouse: receptive field properties and functional organization. Experimental Brain Research 69:594-612.

Migaud M, Charlesworth P, Dempster M, Webster LC, Watabe AM, Makhinson M, He Y, Ramsay MF, Morris RG, Morrison JH (1998) Enhanced long-term potentiation and impaired learning in mice with mutant postsynaptic density-95 protein. Nature 396:433-439.

Mioche L, Singer W (1989) Chronic recordings from single sites of kitten striate cortex during experience-dependent modifications of receptive-field properties. Journal of Neurophysiology 62:185-197.

Missotten $L$ (1965) The ultrastructure of the retina. Brussels: Editions Arscia SA.

Mitchell DE, Sengpiel F (2009) Neural mechanisms of recovery following early visual deprivation. Philosophical Transactions of the Royal Society B: Biological Sciences 364:383-398.

Morales B, Choi S-Y, Kirkwood A (2002) Dark rearing alters the development of GABAergic transmission in visual cortex. The Journal of neuroscience 22:8084-8090.

Morishita H, Hensch TK (2008) Critical period revisited: impact on vision. Current Opinion in Neurobiology 18:101-107.

Morishita H, Miwa JM, Heintz N, Hensch TK (2010) Lynx1, a cholinergic brake, limits plasticity in adult visual cortex. Science 330:1238-1240.

Mrsic-Flogel TD, Hofer SB, Ohki K, Reid RC, Bonhoeffer T, Hübener M (2007) Homeostatic regulation of eye-specific responses in visual cortex during ocular dominance plasticity. Neuron 54:961-972.

Nabel EM, Morishita H (2015) Regulating critical period plasticity: insight from the visual system to fear circuitry for therapeutic interventions. Epigenetic pathways in PTSD: how traumatic experiences leave their signature on the genome:89.

Nakagawa T, Futai K, Lashuel HA, Lo I, Okamoto K, Walz T, Hayashi Y, Sheng M (2004) Quaternary structure, protein dynamics, and synaptic function of SAP97 controlled by L27 domain interactions. Neuron 44:453-467.

Nelson CD, Kim MJ, Hsin H, Chen Y, Sheng M (2013) Phosphorylation of threonine-19 of PSD95 by GSK-3 $\beta$ is required for PSD-95 mobilization and long-term depression. The Journal of neuroscience 33:12122-12135.

Nicoll R, Malenka R (1999) Expression Mechanisms Underlying NMDA Receptor-Dependent Long-Term Potentiation. Annals of the New York Academy of Sciences 868:515-525. 
Nicoll RA, Tomita S, Bredt DS (2006) Auxiliary subunits assist AMPA-type glutamate receptors. Science 311:1253-1256.

Niell CM, Stryker MP (2008) Highly selective receptive fields in mouse visual cortex. The Journal of neuroscience 28:7520-7536.

Niethammer M, Kim E, Sheng M (1996) Interaction between the C terminus of NMDA receptor subunits and multiple members of the PSD-95 family of membrane-associated guanylate kinases. The Journal of neuroscience 16:2157-2163.

Ohki K, Reid RC (2007) Specificity and randomness in the visual cortex. Current Opinion in Neurobiology 17:401-407.

Ohki K, Chung S, Ch'ng YH, Kara P, Reid RC (2005) Functional imaging with cellular resolution reveals precise micro-architecture in visual cortex. Nature 433:597-603.

Olkkola K, Ahonen J (2008) Midazolam and other benzodiazepines. In: Modern Anesthetics 335-360.

Opazo P, Choquet D (2011) A three-step model for the synaptic recruitment of AMPA receptors. Molecular and Cellular Neuroscience 46:1-8.

Opazo P, Sainlos M, Choquet D (2012) Regulation of AMPA receptor surface diffusion by PSD95 slots. Current Opinion in Neurobiology 22:453-460.

Pelvig DP, Pakkenberg H, Stark AK, Pakkenberg B (2008) Neocortical glial cell numbers in human brains. Neurobiology of aging 29:1754-1762.

Philpot BD, Espinosa JS, Bear MF (2003) Evidence for altered NMDA receptor function as a basis for metaplasticity in visual cortex. The Journal of neuroscience 23:5583-5588.

Pizzorusso T, Medini P, Berardi N, Chierzi S, Fawcett JW, Maffei L (2002) Reactivation of ocular dominance plasticity in the adult visual cortex. Science 298:1248-1251.

Prusky G, Douglas R (2004) Characterization of mouse cortical spatial vision. Vision research 44:3411-3418.

Prusky GT, Douglas RM (2003) Developmental plasticity of mouse visual acuity. European Journal of Neuroscience 17:167-173.

Prusky GT, West PW, Douglas RM (2000) Behavioral assessment of visual acuity in mice and rats. Vision research 40:2201-2209.

Prusky GT, Alam NM, Douglas RM (2006) Enhancement of vision by monocular deprivation in adult mice. The Journal of neuroscience 26:11554-11561.

Prusky GT, Alam NM, Beekman S, Douglas RM (2004) Rapid quantification of adult and developing mouse spatial vision using a virtual optomotor system. Investigative ophthalmology \& visual science 45:4611-4616.

Ranson A, Cheetham CE, Fox K, Sengpiel F (2012) Homeostatic plasticity mechanisms are required for juvenile, but not adult, ocular dominance plasticity. Proceedings of the National Academy of Sciences 109:1311-1316.

Rao-Mirotznik R, Harkins A, Buchsbaum G, Sterling P (1995) Mammalian rod terminal: architecture of a binary synapse. Neuron 14:561-569. 
Rittenhouse CD, Siegler BA, Voelker CA, Shouval HZ, Paradiso MA, Bear MF (2006) Stimulus for rapid ocular dominance plasticity in visual cortex. Journal of Neurophysiology 95:2947-2950.

Roberts EB, Meredith MA, Ramoa AS (1998) Suppression of NMDA receptor function using antisense DNA blocks ocular dominance plasticity while preserving visual responses. Journal of Neurophysiology 80:1021-1032.

Rochefort NL, Narushima M, Grienberger C, Marandi N, Hill DN, Konnerth A (2011) Development of direction selectivity in mouse cortical neurons. Neuron 71:425-432.

Rumbaugh G, Sia G-M, Garner CC, Huganir RL (2003) Synapse-associated protein-97 isoformspecific regulation of surface AMPA receptors and synaptic function in cultured neurons. The Journal of neuroscience 23:4567-4576.

Rumpel S, Hatt H, Gottmann K (1998) Silent synapses in the developing rat visual cortex: evidence for postsynaptic expression of synaptic plasticity. The Journal of neuroscience 18:8863-8874.

Rumpel S, Kattenstroth G, Gottmann K (2004) Silent synapses in the immature visual cortex: layer-specific developmental regulation. Journal of Neurophysiology 91:1097-1101.

Sager C, Tapken D, Kott S, Hollmann M (2009) Functional modulation of AMPA receptors by transmembrane AMPA receptor regulatory proteins. Neuroscience 158:45-54.

Sans N, Petralia RS, Wang Y-X, Blahos J, Hell JW, Wenthold RJ (2000) A developmental change in NMDA receptor-associated proteins at hippocampal synapses. The Journal of neuroscience 20:1260-1271.

Sato M, Stryker MP (2008) Distinctive features of adult ocular dominance plasticity. The Journal of neuroscience 28:10278-10286.

Sawtell NB, Frenkel MY, Philpot BD, Nakazawa K, Tonegawa S, Bear MF (2003) NMDA receptor-dependent ocular dominance plasticity in adult visual cortex. Neuron 38:977-985.

Schacter DL, Guerin SA, Jacques PLS (2011) Memory distortion: An adaptive perspective. Trends in cognitive sciences 15:467-474.

Schlüter OM, Xu W, Malenka RC (2006) Alternative N-terminal domains of PSD-95 and SAP97 govern activity-dependent regulation of synaptic AMPA receptor function. Neuron 51:99-111.

Schnell E, Sizemore M, Karimzadegan S, Chen L, Bredt DS, Nicoll RA (2002) Direct interactions between PSD-95 and stargazin control synaptic AMPA receptor number. Proceedings of the National Academy of Sciences 99:13902-13907.

Schuett S, Bonhoeffer T, Hübener M (2002) Mapping retinotopic structure in mouse visual cortex with optical imaging. The Journal of neuroscience 22:6549-6559.

Schwarzkopf DS, Vorobyov V, Mitchell DE, Sengpiel F (2007) Brief daily binocular vision prevents monocular deprivation effects in visual cortex. European Journal of Neuroscience 25:270-280.

Shatz CJ (1990) Impulse Activity and the Patterning Review of Connections during CNS Development. Neuron 5(6):745-756. 
Sheng M, Sala C (2001) PDZ domains and the organization of supramolecular complexes. Annual review of neuroscience 24:1-29.

Sherman SM, Spear PD (1982) Organization of visual pathways in normal and visually deprived cats. Physiological reviews 62:738-855.

Sjöstrand $F$ (1958) Ultrastructure of retinal rod synapses of the guinea pig eye as revealed by three-dimensional reconstructions from serial sections. Journal of ultrastructure research 2:122-170.

Smith GB, Heynen AJ, Bear MF (2009) Bidirectional synaptic mechanisms of ocular dominance plasticity in visual cortex. Philosophical Transactions of the Royal Society B: Biological Sciences 364:357-367.

Sohya K, Kameyama K, Yanagawa Y, Obata K, Tsumoto T (2007) GABAergic neurons are less selective to stimulus orientation than excitatory neurons in layer II/III of visual cortex, as revealed by in vivo functional $\mathrm{Ca} 2+$ imaging in transgenic mice. The Journal of neuroscience 27:2145-2149.

Song I, Huganir RL (2002) Regulation of AMPA receptors during synaptic plasticity. Trends in neurosciences 25:578-588.

Stathakis DG, Hoover KB, You Z, Bryant PJ (1997) Human Postsynaptic Density-95 (PSD95): Location of the Gene (DLG4) and Possible Function in Nonneural as Well as in Neural Tissues. Genomics 44:71-82.

Stein V, House DR, Bredt DS, Nicoll RA (2003) Postsynaptic density-95 mimics and occludes hippocampal long-term potentiation and enhances long-term depression. The Journal of neuroscience 23:5503-5506.

Stent GS (1973) A physiological mechanism for Hebb's postulate of learning. Proceedings of the National Academy of Sciences 70:997-1001.

Stodieck SK, Greifzu F, Goetze B, Schmidt K-F, Löwel S (2014) Brief dark exposure restored ocular dominance plasticity in aging mice and after a cortical stroke. Experimental gerontology 60:1-11.

Sturgill JF, Steiner P, Czervionke BL, Sabatini BL (2009) Distinct domains within PSD-95 mediate synaptic incorporation, stabilization, and activity-dependent trafficking. The Journal of neuroscience 29:12845-12854.

Sugiyama S, Prochiantz A, Hensch TK (2009) From brain formation to plasticity: insights on Otx2 homeoprotein. Development, growth \& differentiation 51:369-377.

Sugiyama S, Di Nardo AA, Aizawa S, Matsuo I, Volovitch M, Prochiantz A, Hensch TK (2008) Experience-dependent transfer of Otx2 homeoprotein into the visual cortex activates postnatal plasticity. Cell 134:508-520.

Sun Q, Turrigiano GG (2011) PSD-95 and PSD-93 play critical but distinct roles in synaptic scaling up and down. The Journal of neuroscience 31:6800-6808.

Suska A, Lee BR, Huang YH, Dong Y, Schlüter OM (2013) Selective presynaptic enhancement of the prefrontal cortex to nucleus accumbens pathway by cocaine. Proceedings of the National Academy of Sciences 110:713-718. 
Syken J, GrandPre T, Kanold PO, Shatz CJ (2006) PirB restricts ocular-dominance plasticity in visual cortex. Science 313:1795-1800.

Tagawa Y, Kanold PO, Majdan M, Shatz CJ (2005) Multiple periods of functional ocular dominance plasticity in mouse visual cortex. Nature neuroscience 8:380-388.

Takesian AE, Hensch TK (2013) Balancing plasticity/stability across brain development. Prog Brain Res 207:3-34.

Tao Y-X, Rumbaugh G, Wang G-D, Petralia RS, Zhao C, Kauer FW, Tao F, Zhuo M, Wenthold RJ, Raja SN (2003) Impaired NMDA receptor-mediated postsynaptic function and blunted NMDA receptor-dependent persistent pain in mice lacking postsynaptic density-93 protein. The Journal of neuroscience 23:6703-6712.

Tomita S, Nicoll RA, Bredt DS (2001) PDZ protein interactions regulating glutamate receptor function and plasticity. The Journal of cell biology 153:F19-F24.

Tomita S, Chen L, Kawasaki Y, Petralia RS, Wenthold RJ, Nicoll RA, Bredt DS (2003) Functional studies and distribution define a family of transmembrane AMPA receptor regulatory proteins. The Journal of cell biology 161:805-816.

Topinka JR, Bredt DS (1998) N-terminal palmitoylation of PSD-95 regulates association with cell membranes and interaction with K+ channel K v 1.4. Neuron 20:125-134.

Tropea D, Kreiman G, Lyckman A, Mukherjee S, Yu H, Horng S, Sur M (2006) Gene expression changes and molecular pathways mediating activity-dependent plasticity in visual cortex. Nature neuroscience 9:660-668.

Vallejo D, Codocedo JF, Inestrosa NC (2016) Posttranslational Modifications Regulate the Postsynaptic Localization of PSD-95. Molecular neurobiology:1-18.

Van Hooser SD (2007) Similarity and diversity in visual cortex: is there a unifying theory of cortical computation? The Neuroscientist 13:639-656.

van Zundert B, Yoshii A, Constantine-Paton M (2004) Receptor compartmentalization and trafficking at glutamate synapses: a developmental proposal. Trends in neurosciences 27:428-437.

Vetencourt JFM, Sale A, Viegi A, Baroncelli L, De Pasquale R, O'Leary OF, Castrén E, Maffei L (2008) The antidepressant fluoxetine restores plasticity in the adult visual cortex. Science 320:385-388.

Wagor E, Mangini NJ, Pearlman AL (1980) Retinotopic organization of striate and extrastriate visual cortex in the mouse. Journal of Comparative Neurology 193:187-202.

Wang B-S, Sarnaik R, Cang J (2010) Critical period plasticity matches binocular orientation preference in the visual cortex. Neuron 65:246-256.

Wang B-S, Feng L, Liu M, Liu X, Cang J (2013) Environmental enrichment rescues binocular matching of orientation preference in mice that have a precocious critical period. Neuron 80:198-209.

Wang C-Y, Chang K, Petralia RS, Wang Y-X, Seabold GK, Wenthold RJ (2006) A novel family of adhesion-like molecules that interacts with the NMDA receptor. The Journal of neuroscience 26:2174-2183. 
Wässle H (2004) Parallel processing in the mammalian retina. Nature Reviews Neuroscience 5:747-757.

Wertz A, Trenholm S, Yonehara K, Hillier D, Raics Z, Leinweber M, Szalay G, Ghanem A, Keller G, Rózsa B (2015) Single-cell-initiated monosynaptic tracing reveals layer-specific cortical network modules. Science 349:70-74.

Wiesel TN, Hubel DH (1963) Single-cell responses in striate cortex of kittens deprived of vision in one eye. J Neurophysiol 26:1003-1017.

Xu W, Schlüter OM, Steiner P, Czervionke BL, Sabatini B, Malenka RC (2008) Molecular dissociation of the role of PSD-95 in regulating synaptic strength and LTD. Neuron 57:248-262.

Yao W-D, Gainetdinov RR, Arbuckle MI, Sotnikova TD, Cyr M, Beaulieu J-M, Torres GE, Grant SG, Caron MG (2004) Identification of PSD-95 as a regulator of dopamine-mediated synaptic and behavioral plasticity. Neuron 41:625-638.

Yasuda H, Barth AL, Stellwagen D, Malenka RC (2003) A developmental switch in the signaling cascades for LTP induction. Nature neuroscience 6:15-16.

Yazaki-Sugiyama Y, Kang S, Câteau H, Fukai T, Hensch TK (2009) Bidirectional plasticity in fastspiking GABA circuits by visual experience. Nature 462:218-221.

Yonehara K, Ishikane H, Sakuta H, Shintani T, Nakamura-Yonehara K, Kamiji NL, Usui S, Noda $M(2009)$ Identification of retinal ganglion cells and their projections involved in central transmission of information about upward and downward image motion. PloS one 4:e4320.

Yoshii A, Sheng MH, Constantine-Paton M (2003) Eye opening induces a rapid dendritic localization of PSD-95 in central visual neurons. Proceedings of the National Academy of Sciences 100:1334-1339.

Zafra F, Hengerer B, Leibrock J, Thoenen H, Lindholm D (1990) Activity dependent regulation of BDNF and NGF mRNAs in the rat hippocampus is mediated by non-NMDA glutamate receptors. The EMBO journal 9:3545.

Zhang M, Xu J-T, Zhu X, Wang Z, Zhao X, Hua Z, Tao Y-X, Xu Y (2010) Postsynaptic density-93 deficiency protects cultured cortical neurons from $\mathrm{N}$-methyl-D-aspartate receptortriggered neurotoxicity. Neuroscience 166:1083-1090.

Zhao J-P, Murata Y, Constantine-Paton M (2013) Eye opening and PSD95 are required for longterm potentiation in developing superior colliculus. Proceedings of the National Academy of Sciences 110:707-712.

Zhu J, Shang Y, Zhang M (2016) Mechanistic basis of MAGUK-organized complexes in synaptic development and signalling. Nature Reviews Neuroscience 17:209-223.

Zuo Y, Lin A, Chang P, Gan W-B (2005) Development of long-term dendritic spine stability in diverse regions of cerebral cortex. Neuron 46:181-189. 


\section{Acknowledgment}

Although only my name is written on the cover of this dissertation, many people have contributed to this work. I owe my gratefulness to all of you who made this possible. Foremost, I am grateful to my supervisor Prof. Dr. Siegrid Löwel for giving me the opportunity to execute this exciting project in her laboratory. Without her constant guidance and advice, this project would not have been possible.

I also would like to thank Prof. Dr. Tim Gollisch for being my second reviewer, member of me thesis committee and for support.

I am grateful for our fruitful collaboration with the laboratory under the direction of Prof. Dr. Dr. Oliver Schlüter, which made this project feasible in the first place. Thank you for being part of my thesis committee and especially for the insightful and constructive comments. Also for giving me access to the laboratory and research facilities. Without the precious support of this work group and especially the contribution from Dr. Huang and Dr. Favaro, it would have not been possible to conduct this research.

I thank all the members of the examination board, Prof Dr. Tobias Moser, Prof. Dr. Ralf Heinrich, and Dr. Ellen Reisinger for agreeing to evaluate my work.

I am glad to be a graduate student of the GGNB (Sensory and Motor Neuroscience program), which is an excellent platform offering diverse method and soft skill courses, excursions, retreats and makes it easy to network with other PhD students and young scientists.

Special thanks to Dr. Bianka Götze and Prof. Dr. Karl-Friedrich Schmidt who introduced me to many methods and always helped me out when necessary.

I am also thankful to the present and former members of the Systems Neuroscience group who created a warm and cordial atmosphere in the lab. Thank you Evgenia, Neethu, Susanne, Justyna, Franzi, Anja, Rashad and Kanishka. Also, I very much appreciate the conscientiously work of Matthias and for excellent animal care. Thank you, Simone, for always cheering me up and for having nice talks, cakes and barbecues. It is great having all of you as colleagues and also for spending nice times together inside as well as outside the lab.

Additionally, I am extremely thankful to the former students for their significant contributions

in this project. Contributions are as following: Leon Hosang for helping in the PSD-93 project and also for being a great lab mate, Kanishka Waghmare and Malte Lübben for helping with the visual water task and Anja, Xiaojie and Plinio for helping with the virus injections. 
I especially thank my sister Dr. Xenia Domes and Dr. Nils Arne Rall for proof reading. That helped me a lot to calm down.

Very importantly, my family and my dear good friends: Thank you so much with all my heart for your love, support, and your time. 


\title{
Sophia Katharina Stodieck
}

\section{Personal Details}

\author{
Name Sophia Katharina Stodieck \\ Telephone No. $\quad+491779151067$ \\ Email_sophia.stodieck@gmail.com \\ Date of birth $\quad 11^{\text {th }}$ of March 1988 \\ Place of birth Munich \\ Nationality German
}

\section{Career}

August 2013 - January 2017

October 2012 - August 2013

October 2010 - October 2012

October 2007 - October 2010

$1998-2007$
PhD student in the Systems Neuroscience Department,

Georg-August- Universität Göttingen at the Göttingen Graduate School for Neurosciences and Molecular Biosciences (GGNB) and Johann-Friedrich-Blumenbach Institute for Zoology and Anthropology, University of Göttingen, Germany

PhD student in the Systems Neuroscience Department, Georg-August- Universität Göttingen

Master of Science in Developmental, Neural and Behavioral Biology, University of Göttingen

Bachelor of Science, Biology, University of Göttingen Annette-von-Droste-Hülshoff Gymnasium (secondary school), Münster finished school with A-levels in Biology, Fine arts, English and 


\section{Publication list}

Huang X*, Stodieck SK*, Goetze B, Cui L, Wong MH, Wenzel C, Hosang L, Dong Y, Löwel S**, Schlüter OM** (2015) Progressive maturation of silent synapses governs the duration of a critical period. Proceedings of the National Academy of Sciences 112:E3131-E3140. *shared first authorship; ** equal contribution

Stodieck SK, Greifzu F, Goetze B, Schmidt K-F, Löwel S (2014) Brief dark exposure restored ocular dominance plasticity in aging mice and after a cortical stroke. Experimental gerontology 60:1-11.

\section{Conference participation}

Sophia K. Stodieck, Xiaojie Huang, Oliver M. Schlüter and Siegrid Löwel (2015) Knock-down of the signaling scaffold postsynaptic density protein95 (PSD-95) in the adult primary visual cortex restored juvenile ocular dominance plasticity. 11th Meeting of the German Neuroscience Society2015, Göttingen, Germany.

Leon Hosang, Sophia K. Stodieck, Bianka Goetze, Xiaojie Huang, Plinio D. Favaro, Oliver M. Schlüter and Siegrid Löwel (2015) The Yin and Yang of cortical plasticity: the role of postsynaptic density proteins 93 and 95 for visual cortical plasticity. 11th Meeting of the German Neuroscience Society

Sophia K. Stodieck, Bianka Goetze, Karl-Friedrich Schmidt, Franziska Greifzu and Siegrid Löwel (2014) Brief dark exposure restored ocular dominance plasticity in ageing mice and after a cortical stroke. $9^{\text {th }}$ FENS (Federation of European Neuroscience) Forum of Neuroscience, Milan, Italy FENS Forum Abstracts 7: 1073.

Sophia K. Stodieck, Bianka Goetze, Franziska Greifzu, Hugo CrucesSolis, Karl-Friedrich Schmidt and Siegrid Löwel (2013) Dark exposure rescues ocular dominance plasticity in the visual cortex of adult mice in an age-dependent manner. 10th Meeting of the German Neuroscience Society 2013, Göttingen, Germany.

\section{Educational activities}

2014 - present

2014
Person in charge for foodsharing.de in Göttingen

Participation at "ELECTRAIN", Göttingen Graduate School for Neurosciences and Molecular Biosciences (GGNB) extended electrophysiology training at the European Neuroscience Institute Göttingen 

Brain, Göttingen

February 2006 Participation at a "SIEMENS Science Camp" organized by "Mathematics \& Sciences Excellence Centre" to the subject: "Light with its diverse nature", Erlangen and Munich

October 2004 Attendance to the event "Mut zu MINT 4 arranged by "Mathematics \& Sciences Excellence Centre" to the subject: "Professional fields and academic study courses in mathematics and sciences", SIEMENS AG, Erlangen

\section{Languages}

German, native speaker

English

Basic knowledge of French

\section{Technical skills}

Microsoft office

Linux

Matlab

Graphpad Prism

Inkscape

\section{Other work experience}

2006 - present $2011-2014$
Hostess, Mystery Shopper and Promoter for i.a. Audi, German Railway, Frankfurter Allgemeine Zeitung, Die Zeit, BAT

Research assistant at the University of Göttingen 